\title{
A 110ヨ 074692
}

UNITED STATES

EPARTMENT OF

BOMMERCE

UBLICATION
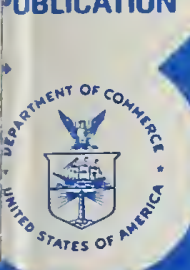

Wational Eurean of Standarās

Library, E-01 hatin. Bidg.

$$
\text { JAN! }=1971
$$

\section{BUILDING SCIENCE SERIES}
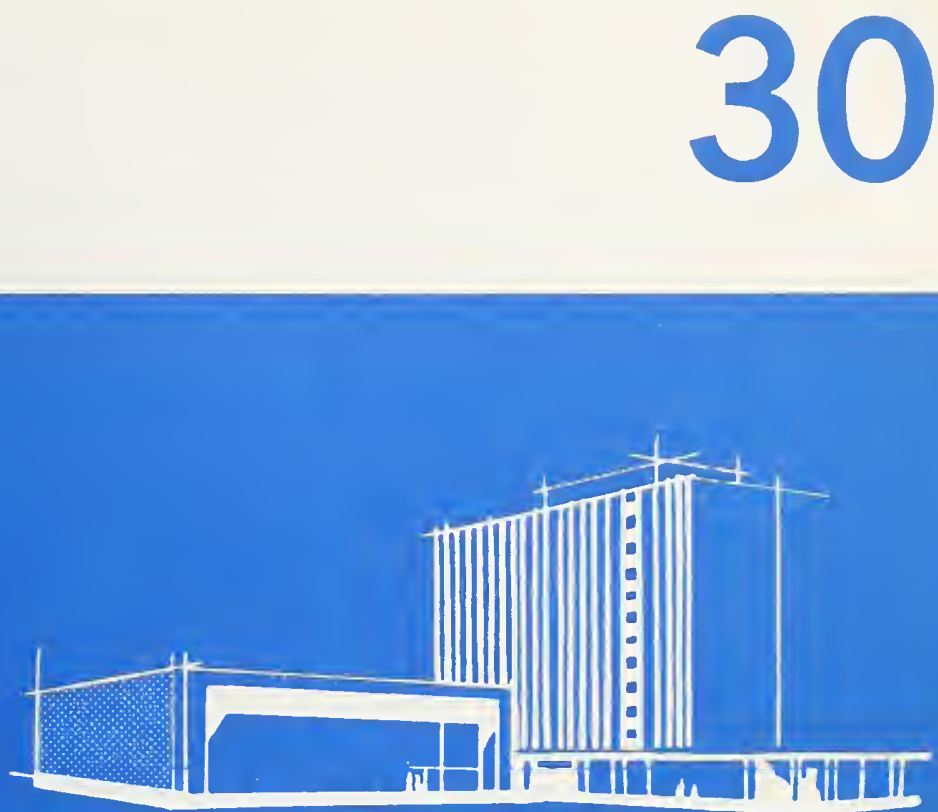

U.S.

DEPARTMENT

OF COMMERCE

National

Bureau of Standards

\section{Wind Loads on Buildings and Structures}




\section{Announcing-The Building Science Series}

The "Building Science Series" disseminates technical information developed at the Bureau on building materials, components, systens, and whole structures. The series presents research results, test methols, and performance criteria related to the structural and environmental funetions and the durability and safety characteristics of building elements and systems.

These publications, similar in style and content to the NBS Building Materials and Structure Reports (1938-59), are directed toward the manufacturing, lesign, and construction segments of the building industry, standards organizations, officials responsible for building codes, and scientists and engincers concelned with the properties of building materials.

The naterial for this series originates principally in the Building Research Division of the NBS Institute for Applied Technolory. Published or in preparation are:

BSSO. Building Research at the National Bureau of Standards. (In press)

BSS1. Ierformance of Buildings_Concept and Measurement. Man and His Shelter. $\$ 2.75$

B.S.2. Interlelations Between Cement and Concrete Properties: Part 1, Materials and Techniques, Water Requirements and Trace Elemerits. 35 cents

BSS3. Doors as Barriers to Fire and Smoke. 15 cents

BSS4. Weather Resistance of Porcelain Enamels: Effect of Exposure Site and Other Variables After Seven Years. 20 cents

BSS5. Interrelations Between Cement and Concrete Properties: Part 2, Sulfate Expansion, Heat of Hydration, and Autoclave Expansion. 35 cents

BSS6. Some Properties of the Calcium Aluminoferrite Hydrates. 20 cents

BSS7. Organic Coatings. Properties, Selection, and Use. $\$ 2.50$

BSS8. Interrelations Between Cement and Concrete Properties: Part 3, Coinpressive Strengths of Portland Cenent Test Mortars and Steam-Cured Mortars. 55 cents

BSS?. Thermal-Shock Resistance for Built-Up Membranes. 20 cents

BSS10. Field Burnout Tests of Apartment Dwelling Units. 25 cents

BSS11. Fire Resistance of Steel Deck Floor A ssemblies. 25 cents

BSS12. Performance of Square-Edged Orifices and Orifice-Target Combinations as Air Mixers. 15 cents

BSS13. Shrinkage and Creep in Prestressed Concrete. 15 cents

BSS14. Experimental Determination of Eccentricity of Floor Loads Applied to a Bearing Wall. 15 cents

BSS15. Interrelations Between Cement and Concrete Properties: Part 4, Shrinkage of Hardened Portland Cement Pastes. 75 cents

RSS16. Techniques for the Survey and Evaluation of Live Floor Loads and Fire Loads in Modern Office Buildings. 40 cents

BSS17. Causes of Variation in Chemical Analyses and Physical Tests of Portland Cement. 40 cents

BSS18. Smoke and Gases Produced by Burning Aircraft Interior Materials. 35 cents

BSS19. A Study of the Variables Involved in the Saturating of Roofing Felts. 30 eents

BSS20. Proceedings of a Seminar on the Durability of Insulating Glass. 75 cents

BSS21. Algorithms for P'sychrometric Cal'ulations. 55 eents

BSS22. Investigation of Perfumance Characteristies for Sanitary Plumbing Fixtures. 70 cents

BSS23. Hail Reststance of Roofing I'roducts. 25 cents

BSS24. Natural Weathering of Mineral Stabilized Asphalt Coatings on Organic Felt. 30 cents

BSS25. Structural Performance Test of a Building System. $\$ 1.25$

BSS26. Radiation Errors in Air Ducts Under Nonisothermal Conditions Using Thermocouples, Thermistors, and a Resistance Thermometer. 25 cents

BSS27. Performance of Louvered Devices as Air Mixers. 30 cents

BSS28. Jisploratory Studies of Farly strength Ievelopment in Portland Cement Pastes and Mortars. 2.5 eents

BSS29. 1064 Exposure Test of I'orcelain Enamels on Aluminum-Three Year Inspection. 25 cents

Send orders wtih remittance to: Superintendent of Documents, U.S. Government Printing Office, Washington, D.C. Remittances from foreign countries should include an additional one-fourth of the purchase price for postage. 


\title{
Proceedings of Technical Meeting Concerning Wind Loads on Buildings and Structures
}

\author{
Held at the National Bureau of Standards \\ Gaithersburg, Maryland, January 27-28, 1969 \\ R. D. Marshall, Editor \\ Building Research Division \\ Institute for Applied Technology \\ National Bureau of Standards \\ Washington, D.C. 20234 \\ and \\ H. C. S. Thom, Editor \\ Environmental Data Service \\ Environmental Science Services Administration \\ Silver Spring, Md. 20910
}

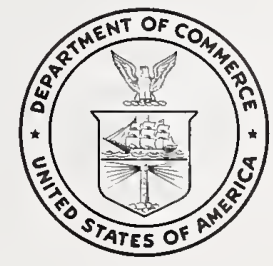

Building Science Series 30

Nat. Bur. Stand. (U.S.), Bldg. Sei. Ser. 30, 169 pages (November 1970)

CODEN: BSSNB

Issued November 1970 


\begin{abstract}
A technical meeting concerning wind loads on buildings and structures was held at the Gaithersburg Facility of the National Bureau of Standards on January 27 and 28, 1969. The meeting, jointly sponsored by the Building Research Division of the National Bureau of Standards and the Environmental Data Service of the Environmental Science Services Administration, was intended to promote the exchange of information between research workers and practicing engineers engaged in this important area of technology.

Thirteen papers covering four themes were presented. These themes were as follows: engineering problems in the design of structures to resist wind loads; atmospheric considerations; experimental and theoretical determination of aerodynamic forces; and recent advances in design procedures and current deficiencies. Presentations were followed by a panel discussion which included a summary of each theme. The last session of the meeting was devoted to open discussion.
\end{abstract}

Key Words: Aerodynamic forces; atmospheric boundary layer; structural design; turbulence; wind effects; wind loads. 


\section{Foreword}

The effeets of wind on buildings and struetures have always presented engineers with a formidable ehallenge. That attempts to adequately provide for these effeets within reasonable eeonomie bounds have been only partially sueeessful is evideneed by the number of struetural failures direetly attributable to wind aetion. While mueh has been learned from past failures, the eurrent rate of innovation in building materials and eonstruetion methods, eoupled with the trend toward extremely slender structures, has ereated new demands for knowledge of wind loads. Although the prevention of eatastrophic failures must be given priority, the introduction of performanee coneepts has foeused new attention on other effects such as rain penetration, aeolian noise produetion and the physiologieal implieations of building motions. The effects of buildings on loeal wind environment are of great importanee in the proper design of aneillary items sueh as eourtyards, plaza areas and entryways.

In eomparison to other areas of engineering teehnology, progress in assessing wind effeets has been remarkably slow. This ean be aseribed to the interdisciplinary nature of the problem whieh encompasses meteorology, fluid mechanics and struetural engineering. That a conferenee entirely devoted to the subject of wind effeets on buildings and structures could provide a ehannel of communication between researehers in these three separate areas was demonstrated by the symposium at Teddington, England in 1963. This was followed 4 years later by an equally suecessful researeh seminar at Ottawa, Canada. Here in the United States, the time had elearly eome for a meeting of national seope where representatives from universities, eonsulting firms, industry and government eould exehange information and ideas.

The proeeedings that follow represent a signifieant contribution to the understanding of wind effeets on buildings and struetures. For their papers and active participation that made the meeting highly sueeessful, our thanks are extended to all the speakers.

$$
\text { Washington, D.C. }
$$

R. D. Marshall

H. C. S. Thom

Conference Co-Chairmen 



\section{Contents}

Welcome to NBS: A. V. Astin, Director, National Bureau of Standards

Session 1. ENGINEERING PROBLEMS IN THE DESIGN OF STRUCTURES TO RESIST WIND LOADS

Chairman: Dr. A. Allan Bates, Chief, Office of Engineering Standards Liaison

National Bureau of Standards

Design and Construction for What Wind Loads and Why

L. C. Maugh

University of Michigan

Design Wind Loads for Building Wall Elements

Wayne F. Koppes, AIA

Architectural Consultant . . . . . . . . . . .

Session 2. ATMOSPHERIC CONSIDERATIONS

Chairman: Mr. H. C. S. Thom, Senior Research Fellow

Environmental Data Service

Guidelines in Selecting Wind Measuring Instruments and Their Locations for Wind

Loading Studies

Gerald C. Gill

University of Michigan

The Adequacy of Existing Meteorological Data for Evaluating Structural Problems Irving A. Singer and Maynard E. Smith

Brookhaven National Laboratory.

The Characteristics of Atmospheric Turbulence as Related to Wind Loads on Tall

Structures

George H. Fichtl, John W. Kaufman, and William W. Vaughan

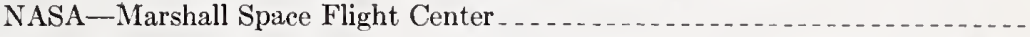

Session 3. EXPERIMENTAL AND THEORETICAL DETERMINATION OF AERODYNAMIC FORCES

Chairman: Professor A. G. Davenport, University of Western Ontario

Fluctuating Moments on Tall Buildings Produced by Wind Loading

J. E. Cermak, W. Z. Sadeh, and George Hsi

Colorado State University

Experience with Wind Pressure Measurements on a Full-Scale Building

W. A. Dalgliesh

National Research Council of Canada

Division of Building Research

Influence of Architectural Features on the Static Wind Loading of Buildings

Hans J. Leutheusser

University of Toronto.

The Unsteady Surface Pressure Around Circular Cylinders in Two-Dimensional Flow Wayne E. Simon

Martin-Marietta Corporation.

On the Reliability of Gust Loading Factors

Barry J. Vickery

University of Western Ontario

Session 4. RLCENT ADVANCES IN DESIGN PROCEDURES AND CURRENT DEFICINNCIES

Chairman: Mr. Edward Cohen, Partner, Ammann \& Whitney,

The Treatment of Wind in the Design of Very Tall Buildings

L. E. Robertson and P. W. Chen

Skilling, Helle, Christiansen, Robertson _. . . _ _.

Dynamic Response of Tall Flexible Structures to Wind Loading

Joseph Vellozzi and Edward Cohen

Ammann \& Whitney.

Some Deficiencies in Current Methods of Analysis

R. W. Clough

University of California at Berkeley 


\section{Contents-Continued}

PANEL DISCUSSION-Session Chairmen -

Combining a Wind Tunnel Analysis With a Three-Dimensional Analytic Building Analysis

Gary C. Hart

University of California at Los Angeles

The Engineering Interpretation of Weather Bureau Records for Wind Loading on Structures

S. C. Hollister

Cornell University. 


\title{
WELCOME TO NBS
}

\author{
Dr. Allen V. Astin, Director* \\ National Bureau of Standards \\ Washington, D.C. 20234
}

It is a great pleasure for me to welcome you to this meeting on the effect of wind loads on buildings. We are pleased with the opportunity of being able to join with the Environmental Science Services Administration in the planning for this symposium, and actually cooperating with them in some important studies.

I would like to take advantage of my opportunity to say a few words about the National Bureau of Standards, since this may be the first time a number of you have been here. We at NBS are concerned mainly with advancing and exploiting the science of physical measurement. Vannevar Bush, the distinguished American scientist-engineer, said in the Foreword he wrote to the recently published Measures for Progress: A History of the National Bureau of Standards the following:

"If men working together are to accomplish anything useful whatever, they must, above all, be able to understand one another. That is the reason for a National Bureau of Standards."

We attempt to provide the basis for the language for meaningful, compatible exchange of quantitative information among scientists and engineers. This program ranges from providing standards for the basic physical quantities, to the determination of standard test methods for measuring the properties of materials, mechanisms and structures. Our program on measurements runs, of course, from the physical quantities to complex artifacts which our society uses. One of the most important areas in this business of how to measure the properties of artifacts meaningfully is our building research program. In this area, we have been trying over the past few years to extend our test methods from those aimed at determining design characteristics of components to looking at buildings as systems, and developing critcria for the evaluation of the performance of the system in meeting the social and technological needs for which the system is intended. We then develop test methods to permit the determination of conformance or nonconformance to such criteria. This cmphasis on performance, using more and more the systems approach to building problems, has led to a real appreciation of the fact that we need to know more about how to design buildings to resist wind loads. And, in cooperation with ESSA, we have recently initiated a program on this. Of course, the whole problem is the one which you intend to explore during your meeting here. I am pleased to welcome some of our Canadian neighbors to this meeting. The Canadian building reserach program has long been one which we have respected, and, in many respects, it has served as a model for some of the things we have tried to do here at the National Bureau of Standards. It's a pleasure to have you with us. I hope you enjoý your meeting. Thank you very much.

* Retired. 



\title{
SESSION 1.
}

\section{ENGINEERING PROBLEMS IN 'THE DESIGN OF S'TRUCTURES TO RESIST WIND LOADS}

\author{
Chairman: Dr. A. Allan Bates \\ Chief, Office of Engineering Standards Liaison \\ National Bureau of Standards \\ Washington, D.C. 20234
}





\title{
DESIGN AND CONSTRUCTION FOR WHAT WIND LOADS AND WHY
}

\author{
L. C. Maugh \\ Professor of Civil Engineering \\ University of Michigan \\ Ann Arbor, Michigan 48104
}

\begin{abstract}
This paper is primarily concerned with the design of various structures for wind forces from the viewpoint of the average structural engineer. The subject matter focuses attention upon thrce important types of structural problems whose solutions are still so uncertain as to require considerably more study and research. These problems are:

1. Wind action upon structural frameworks during construction with emphasis upon the relatively large number of partial or complete failures.

2. The behavior of completed structures of relatively high frequencies when subjected to wind loads. An important factor here is the amount and characteristics of motion in high-rise buildings that will be accepted by the occupants.

3. Some dynamic problems associated with wind forces acting upon structures of low frequencies such as tall chimneys and guyed towers.

The above problems are discussed from the need for better design criteria and specifications as well as more exact mathematical analysis and more refined wind measurements. The importance of the latter factor is recognized but the many empirical factors in structural design are also considered in this paper.
\end{abstract}

Key words: Buildings; design criteria; dynamics; gust factors; smoke stacks; structural failure; wind loads.

\section{Introduction}

Although many interesting papers and research reports on the magnitude and distribution of wind forces on various types of structures have been published, I will limit my remarks to the practical use of this information by structural designers. For purposes of presentation rather than order of importance, I will discuss the action of wind forces upon structures in terms of the following problems.

1. Wind action upon structural frameworks during construction with emphasis upon the relatively large number of partial or complete failures.

2. Wind action upon completed structures of relatively high frequencies.

3. Some dynamic problems associated with wind forces acting upon structures of low frequencies.

\section{Wind Action Upon Frameworks During Construction}

I have always been interested in the number of bridges and buildings that have collapsed or have been severely damaged during construction by moderate to severe wind storms. Usually the description of such failures in technical literature is not sufficiently complete as to assign exact reasons for the disasters. I might add that a careful examination of the debris does not always solve the problem either.

As an example of the problem just mentioned, I will quote from a recent article in the Engineering News Record, December 12, 1968, as follows, "Winds Topple Bolted Steel Frame-Winds gusting to $80 \mathrm{mph}$ during a 20 -minute snow and rain squall last week toppled a steel frame being erected for a four-story office building in Greenwich, Connecticut. About 15 workers, warned by swaying that preceded the collapse, escaped the frame and injury." A further quotation gives, "The frame of a similar, larger structure had been completed and was not damaged." To me, the significant part of these statements is: "warned by swaying that preceded the collapse" which is certainly a declaration of dynamic magnification of the internal stresses. Also, the statement that a larger completed structure was not damaged is both interesting and typical.

In many cases, I believe, an important reason for construction failures in two to four story buildings goes back to a disregard of a provision in the codes which, as in the AISC Specifications [1]* states, "Proper provision shall be made for stress

\footnotetext{
* Figures in brackets indicate the literature rcferences at the end of this paper.
} 
caused by wind during erection and after completion of the building. The wind pressure is dependent upon the conditions of exposure, geographical location, and shape of the structure." Any practical designer is aware of the fact that the size of primary structural members in low buildings is seldom affected by wind forces because of the permissible increase in working stresses when wind stresses are included in the total. He therefore tends to minimize the importance of the wind design and will often disregard what might happen to the framework during construction. In my opinion, the statement given in the Specifications requires that the designer should study the wind problem during construction and that provision should be made to provide some permanent internal bracing if necessary.

The primary factors that contribute to construction failures during wind storms are as follows:

(a) No internal bracing that might insure safety during construction is specified by the designer.

(b) No plans for temporary bracing are furnished by the contractor and consequently are not checked by a competent structural engineer before installation. Also the installation is not inspected as the construction proceeds.

(c) No consideration is given to the dynamic loads and properties of the structural framework during erection. Certainly the shape factors of the various structural elements, the total exposed areas, a possible resonant condition of first mode vibration due to a wind forcing function, together with the low buckling strength of the unbraced beams and columns should be considered. These problems all add up to trouble.

As an illustration of designing for erection, I recall the study that I helped make for the construction of a $325 \mathrm{ft}$ high gunited steel stack. To guard against lateral vibration and ovalling of the crosssection, it was specified on the plans that the steel erection should proceed in three stages, in the following manner. When the steel shell with ring stiffeners reached the height of $190 \mathrm{ft}$ above its base, erection must stop until that portion of the stack is completely gunited and cured; erection may then proceed for another $60 \mathrm{ft}$ and then stopped for guniting and curing to that elevation; the remainder of the stack was then erected. On another similar stack only two stages of erection and guniting were used.

If I appear to be overemphasizing wind problems during construction, it is not only because of the loss of life and property, but also because of the opportunity that such problems offer to clearly visualize the fundamental principles of wind action that are associated with practically all types of structures; principles that will be considered in more detail by other speakers at this conference.

\section{Wind Action Upon Completed Structures of Relatively High Frequencies}

Wind problems in completed high rise buildings have increased with the modern trend toward the use of flexible partitions, large window areas, and combinations of elements of different materials. The increased use of lightweight concrete deserves particular mention because of the wide variations in its physical properties. With respect to various types of structural frameworks currently in use, it is difficult to interpret, in terms of structural behavior, such designations as: internally braced frames, externally braced, core braced, unbraced, shear walls, and partial shear walls. Present trends toward the stiffness method of analysis using complex computer programs that involve the displacements of the entire structure are appealing in terms of mathematical exactness, but in terms of the actual design, I still favor more limited computer programs that will provide information with regard to the relative stiffness of selected structural combinations of elements. With this information the designer can assign reasonable paths for the transmission of wind forces through the structure and can make changes as the design progresses. When the final selection of the structural elements approaches completion then more sophisticated analytical programs can be applied. However, if the preliminary design is made by experienced designers, I am sure that no significant structural changes will be required.

In structural design the function of the structure must occupy a position of importance commensurate with safety. Therefore, in most high rise buildings the movement must be controlled so that it is acceptable to the occupants. Unfortunately, I have no reliable information as to the magnitude and periods of motion that will be accepted by either short time or long time occupants. Perhaps some practical tests upon a considerable number of people can be conducted so that the motion and conditions will be characteristic of buildings. I recall a pedestrian bridge that I designed which received some critical comments from people who were accustomed to walking over bridges that were designed for a series of heavy trucks. This experience convinced me that most people are very sensitive to structural motion that can initiate fear. To take this fear emotion into 
consideration, it may be necessary to conduct the tests at or near the top of a tall building.

To determine the motion of a high rise building additional information is required concerning the magnitude, distribution, and the time variation of wind pressures upon exterior walls, both windward and leeward. This information will require full scale tests upon buildings of different shapes and the correlation of the results with small seale wind tunnel tests. The need for additional tests is exemplified by the recent report of wind pressures on an 18 story office building by Newberry, Eaton, and Mayne entitled "The Nature of Gust Loading on Tall Buildings" [2]. This experimental work on a rather ordinary rectangular type building was performed in London, England, under the direction of the British Research Station. Even though familiar to many of you, a few quotations from this report may be of interest, such as: "Under these winds it is found that the whole load is carried by the windward face of the building, the load on the leeward face being negligible. The short duration structural loads are about $60 \%$ greater than the one minute averages that have been the basis of many codes of practice, but the overall drag coefficient in the natural wind appears to be less for gust loading conditions than that assumed in wind load calculations based on model tests. These two effects largely balance each other." Obviously, the selection of gust loadings must be related to the location, shape, and period of the building, but from the results of tests upon actual structures, many engineers will question the need for providing this information by complicated equations with many uncertain parameters. An important question is, therefore, can we not aim at improving our building codes and still keep them relatively simple to apply?

\section{Structures With Relatively Low Frequencies}

Several types of structures with rather large fundamental periods of vibration, say from 1 to 6 seconds, require more extensive investigation of the vibration due to wind forces. I will mention here only the wind action upon unbraced tall stacks and guyed steel towers. In a recent paper [3] on the "Dynamic Design of Reinforced Concrete Chimneys," my colleague, Dr. W. S. Rumman, and I presented recommendations for calculating internal stresses due to resonant wind and seismic vibration. From about 18 years of experience in the design of tall tapered reinforced concrete chimneys with circular crosssections, we believe that the following statements from our paper in regard to design criteria for vortex action are both conservative and sufficiently accurate: "However, the assumption that inaximum amplitudes will be obtained when there is sustained periodicity of the vortex action with the same period as the chimney is on the safe side and will be accepted as a reasonable basis for estimating the maximum lateral wind moments and shears. Furthermore, for tapered chimneys, experimental results indicate that the resonant wind velocity is influenced primarily by the upper portion."

In our numerical calculations the following values are commonly assumed: a Strouhal number of 0.2 , a controlling diameter at about one-sixth of the height from the top, a damping ratio of 0.04 of critioal, a coefficient of lift $C_{L}$ of 0.66 , and a sinusoidal variation of the lateral pressure with respect to timo. Obviously, a design based upon such assumptions must be regarded as primarily empirical. I am sure that the designs based upon these recommendations are overly conservative but, as the cost of a chimney is affected very little by this conservatism and since outage time of generators is very costly, I hesitate to suggest any reductions until more reliable information is obtained. One certain thing about the problem is that chimneys will be built whether the problem is completely solved or not. More research work upon the behavior of actual chimneys is needed to check the results of small scale wind tunnel tests, I hope that such full scale tests will be made in the near future.

The design and construction of tall guyed towers involves the solution of many special problems among which the vibration of the guy cables due to wind action is one of the most important and difficult. Solutions to this problem have been discussed by Klousek [4] in 1947, by Davenport [5] in 1959 and 1965, and just recently, by H. Sarvi [6] in a dissertation at the University of Michigan. More observations upon actual towers will be needed to verify the accuracy of these mathematical solutions when applicd to ice covered cables subjected to constantly varying wind gusts.

\section{Summary}

I have presented only a few examples of the problems which the structural designer must consider in designing buildings, bridges, towers, and chimneys for wind forces. The question arises, what basic information concerning wind forces does the designer really need to make an adequate design? Although I have made no survey of professional 
opinions on this subject, my own experience suggests the following basic material.

1. A careful study of the final report [7] on "Wind Forces on Structures" by the Task Committee on Wind Forces, Structural Division, ASCE, Trans., Vol. 126, 1961. This paper gives basic information on the nature of wind forces in terms with which the structural engineer is familiar.

2. An understanding of the calculation of the basic velocity pressure from the recommended fastest-mile wind velocity for the geographical location of the site and the modification of this pressure for height and terrain as recommended in the November 1965 proposed revision of the USASI Standard A58.1-1955.

3. Equally important is a careful study of gust factors, together with shape and pressure coefficients for modifying the basic wind pressures. The designer should avoid a routine selection of such data from one code but rather compare the information with similar codes in other countries. The differences may be quite interesting.

4. For ordinary buildings, incorporating the dynamic response of the structure into formulas for gust effect should be considered as supplementary material and not made a basic part of a building code. In other words, the use of such material should be optional.

5. The designer should have a good understanding of the variation of positive and negative pressures over the surfaces of the walls and members; also the rapid variation of these pressures, particularly the negative, with respect to time.

6. When the structure is an unusual one, the designer should seek more information from experts in the field of wind action. However, his responsibility for the safety and performance of the structure can not be delegated.

\section{References}

[1] American Institute of Steel Construction "Manual of Steel Construction," 1963, Sec. 1.3.5, p. 5-13.

[2] Newberry, C. W., Eaton, K. J., and Mayne, J. R., "The Nature of Gust Loading on Tall Buildings," Current Paper 66/68, Building Research Station, Garston, Hertfordshire, England.

[3] Maugh, L. C., and Rumman, W. S., "Dynamic Design of Reinforced Concrete Chimneys," Journal of the Amer. Concrete Inst., Sept. 1967, Vol. 64, pp. 558-567.

[4] Klousek, V., "Solution Statique et Dynamique des Pylones d'Antenne Haubanes," Publication, International Association for Bridge and Structural Engineering, Vol. VIII, 1947.

[5] Davenport, A. G., "Dynamic Behavior of Massive Guy Cables," Proceedings, ASCE, No. ST2, 1965.

[6] Sarvi, Hassan, "Dynamic Behavior of Guy Cables Subjected to a Small Periodic End Disturbance," Doctoral Dissertation, The University of Michigan, 1968.

[7] ASCE Task Committee on Wind Forces, "Wind Forces on Structures," Trans. ASCE, V. 126, Part II, 1961, pp. 1124-1198. 


\title{
DESIGN WIND LOADS FOR BUILDING WALL ELEMENTS
}

\author{
Wayne F. Koppes, AIA \\ Architectural Consultant \\ Basking Ridge, New Jersey 07920
}

\begin{abstract}
It is principally the structural engineer who has been concerned with wind forces acting on buildings, because it is his responsibility to design the structure to resist their overall effect. Recently, however, the importance of localized wind luads acting on the surfaces of buildings has also been reeeiving much attention. These local loads affect wall design, and are a matter of conceln to both the architect and the manufacturer of wall parts.

The important differences between overall and loeal wind loads include magnitude of intensity, character, period of duration, significance in relation to building size and shape, and importance in respect to potential property loss. Complete destruction of buildings by wind action is rare, but local failures are not uncommon and, on the whole, are mole costly.

Because gust and shape factors are highly unpredictable, increasing reliance is being placed on wind tunnel tests and measurements on aetual buildings. Both confirm that negative pressures usually exceed positive pressures on parts of wall areas. Few building codes in this country recognize this, in speeifying design wind loads for walls, though codes in some other countries do. The importance of terrain conditions is not reflected, either, in code provisions.

Failures of windows and other wall elements due to wind loads occur rather frequently, but relatively few of them are documented. Several interesting examples of such failures ean, however, be cited.

Another important aspect of local wind effects is the funneling of ten experienced near the base of tall buildings. Notable examples of this phenomenon have bcen obscrved, and research studies directed at their causes have produced significant findings.

Codes and standards cannot be expected to provide guidance for designers in respect to unique local wind effects, but they should provide information as to loads which wall surfaces of typical buildings will likely experience. The only standard currently providing this kind of data is the Tentative Standard for Design Wind Loads on Walls of Rectangular Buildings, published by the National Association of Architectural Metal Manufacturers. The background, purpose and features of this Standard are noteworthy, and it is serving a useful purpose.

A review of current investigations of local wind loads, both by wind tunnel tests and field measurements, indicates that certain common assumptions regarding their intensity and distribution on building surfaces should be reexamined. Further information regarding the nature of these loads is urgently needed by, and should be morc promptly disseminated to, the designers and producers of building wall systems.
\end{abstract}

Key words: Buildings; fluctuating pressure; gust factors; walls; wind damage; wind loads.

Traditionally, it has been chiefly the structural engineer who has been eoneerned with the influence of wind on building design, because it is his responsibility to insure the stability of the strueture against eolleetive wind pressures. It is this overall effeet, I believe, whieh is still of prime interest to most engineers, and perhaps to most of this audienec, too. During the past deeade or so, however, there has developed an inereasing eoncern also with what I shall refer to as the local wind effeets, as eontrasted to its overall effects, those wind-induced loads acting on all local areas of the building surfaces and affeeting wall design. Although these local effects are of course related to the overall effects, there are some important differences between the two.

It should be reeognized that in most eases the overall effeets and the local effects represent different areas of design responsibility. While it is the struc- tural engineer who is responsible for the "bones" of the structure, it is usually the architeet who designs its "skin." When walls are composed of faetorymade units, as is common nowadays, the manufacturers of such units, too, are necessarily eoncerned with local wind effects. All of those components which form the envelope of the building must have adequate strength and stiffness to resist the loads to which they will be subjected. Thus there are three parties, not only the engineer but also the arehitect and the wall eomponent manufacturer, who share design responsibilities, and two of these three parties are more concerned with local wind loads than with the overall wind effects.

The purpose of this paper, therefore, is to stress the importanee of local wind loads in wall design. Being in no position to eontribute any new knowledge in this area, I shall attempt simply to summarize 
current practice and problems, indicate some seeming inconsistencies and perhaps identify some information gaps. Rather than presuming to suggest answers, I shall probably be raising some questions, in the hope that others may wish to offer opinions on some of the matters discussed.

Perhaps one of the most important contributions to the concern with local wind loads was the series of pressure coefficient values for variously shaped buildings and structures published in 1956 by the Swiss Association of Engineers and Architects. Parenthetically, the fact that architects apparently were involved in this work tends to substantiate some of my earlier observations. Since 1961, Canadian authorities have referenced these coefficients, along with some of their own derivation, in that part of their National Building Code dealing with design wind loads. I know of no major building code in this country which has provided such information as a guide to the designers of building walls.

In my view, the important differences between the overall loads, which must be resisted by the bones of the structure, and the local loads which act on the skin, are these:

1. The overall load is the net result of all loads occurring simultaneously at any time on all building surfaces, whereas the local load is the peak load which may occur at any time at any location on any surface.

2. Generally, only gusts of perhaps 2 seconds or more duration are significant in determining the critical overall loading, but the critical local load is momentary in nature.

3. Overall loads are seldom of much concern on low buildings of large area, whereas local loads are significant on buildings of all shapes and sizes.

4. Local loads are influenced by the configuration of the buildings surface on which they act, to a much higher degree than are the overall loads.

5. The most critical local loads are usually negative in character, whereas the overall load represents a combination of positive and negative pressures.

6. Maximum local loads are usually of greater intensity than the overall design load.

How about the relative significance of the local and overall design wind loads in terms of potential damage or loss resulting if they are underestimated? Certainly the results of inadequate provision for the overall load acting on a building of consequence might well be catastrophic, as compared with the loss of its windows or parts of its walls. Fortunately, as far as I know, this hasn't happened. Tower structures and bridges have failed under wind loading, and of course buildings have been destroyed in tornados, but I've heard of no important building having been lost in a lesser windstorm. Some say this may be because they're all over designed. On the other hand, we all know of instances where windows have been blown out, or wall elements have been blown off of buildings in high winds. While the complete destruction of a building by wind action is a very rare occurrence, local failures, especially to roofs, windows and wall cladding, are not uncommon and, in the aggregate, are much more costly. It must be remembered, too, that when extensive wind damage does occur, it usually begins with some small local failure.

Any design wind load, whether overall or local, is generally considered to be equal to the velocity (or stagnation) pressure at a height of 30 feet or 10 meters, modified by a height factor, a gust factor and a shape factor. In theory, this seems rather simple, but in practice the determination of the actual wind pressure existing at a given point on the surface of a building, even if the velocity is known, is a complex problem, if indeed it's possible at all. This is due largely to the fact that two, at least, of the modifying factors - the gust factor and the shape factor, or pressure coefficient-vary with circumstances and are highly unpredictable. They change not only with the wind direction, but also the nature of the surroundings and the configuration of the building surface itself. The best we can hope for is a reasonable approximation, not of the actual wind load, but of the probable maximum load that may be expected under given conditions, over a given period of time.

It is such uncertainties that have led to increasing reliance on two methods of obtaining more accurate information about wind action on buildings. One of these is model testing in boundary layer wind tunnels, where surrounding conditions can be simulated and all wind directions can be studied; the other is the measurement of actual wind forces on real buildings. Both methods have their advantages and their limitations, as I'm sure you'll be hearing from other speakers who are experienced and expert in their use. My impression, as decidedly a non-expert, is that in the United States we have done some significant work in model testing but very little work in field measurements. Other countries, however, notably Canada, England and Denmark, have done and are doing important work in this latter area. 
To those concerned with wall design, one of the most important facts established by these studies is that the local negative, or suction loads, are much higher then previously suspected. They appear generally to be at least equal to, and often greater than, the positive loads, and on corner areas of tall buildings may be several times as high. I strongly suspect, though, that most architects don't know this, because they haven't been informed. With the exception of those relatively few who have been involved in the design of our more important buildings, I believe that the majority of architects, and maybe even a few engineers, overlook the importance of negative loading on windows and walls. Very rarely are windows blown into a building in high winds; nearly all of the wind clamage to buildings is due to glass being sucked out of its frame, or the roofing, cladding or awnings being blown away from the structure.

Unfortunately, few of the results of research, and not enough of the current knowledge regarding wind action has been translated into practical working guidelines for the architect's use. Generally, his only guidance in respect to design wind loads is the building code under which he is operating. There are four nationally recognized codes which govern most of our building other than in the largest cities, most of which have their own codes. These four are the so-called National Building Code, promulgated by the American Insurance Association, the BOCA Code, by the Building Official's Conference of America, the Southern Building Code and, on the west coast, the Uniform Building Code. Three of these codes call for design wind loads ranging, with height, from 15 to $40 \mathrm{psf}$, though the Southern Code specifies also loads 10 pounds higher for coastal regions. The Uniform Code, 1967 edition, specifies the same design loads as given in the 1955 edition of ASA Standard A58.1, ranging from 15 to 100 psf. Two of the codes specify that exterior walls shall be capable of withstanding these loads acting either inwardly or outwardly, one calls for negative loads somewhat less than positive loads, and one makes no mention at all of negative loads. The recently adopted code for New York City has perhaps wisely not attempted to completely define design wind load requirements. Although it calls for overall loads ranging, with height, from 15 to $40 \mathrm{psf}$, with a minimum of $30 \mathrm{psf}$ for glass and panels, the local loads specified are 30 psf positive or 20 psf negative for all heights up to 500 feet, with a requirement that local design loads for greater heights be specially investigated by engineering analysis. It's quite apparent that there are confusing inconsistencies in torlay's building corle requirements, especially with regard to local design loads, and it's understandable, I think, that the architect is often uncertain as to how exterior walls should be designed.

The building codes of some other countries are more explicit. The Canadian Code, by listing pressure coefficients for various building shapes, calls for negative pressures ranging from $7 / 9$ to 1.7 times the maximum positive pressures. The Danish Code specifies shape factors, or pressure coefficients, for rectangular buildings in terms of a formula which takes into account the proportions of the building, and also results in maximum negative loads, particularly near the corners of tall buildings, which are about $13 / 4$ times the maximum positive loads. I know of no codes in this country which provide similar helpful guidance to the designer of walls.

Due in large part to Professor Davenport's work, we have come to recognize that the rate of increase of wind velocity with height above the ground varies with the type of terrain over which the wind blows. Obviously there is an infinite variety of terrain conditions, but for practical reasons these must be reduced to a few categories representing major differences. I believe that generally three types of terrain, or types of exposure, as I prefer to think of them, are accepted as adequately representative:

Type A, which, in general terms, is an urban or protected area,

Type B, which is a suburban area, a wooded area or rolling terrain, and

Type C, which is flat open country or the shoreline of a large body of water, fully exposed to a long fetch of wind.

I'm sure that most of you are much more knowledgeable than I am about terrain effects and the theory associated with velocity profiles, so I don't propose to expound on these nratters. As a layman, however, I would like to offer several observations bearing on the subject. The first is that although this important concept has been amply proven and widely accepted for some time, no major building code, as far as I know, has yet recognized it. However, the fortheoming USASI Standard-the updated edition of ASA A58.1-will, I understand, specify design wind loads according to type of terrain, and the NAAMM Standard for Design Wind Loads on Walls, which I'll describe later, is also based on this concept. 
My second observation is that, although wind loads are undoubtedly affected by the type of terrain or building exposure, there may be some doubt, at least in my mind, as to whether we should be thinking in terms of three types of exposure or perhaps only two. The Type $\mathrm{C}$ exposure, which is the most severe, is easily identified, and any building erected along the seacoast is very likely to remain exposed to a long fetch of wind throughout its lifetime. But the distinction between Type A and Type $B$ exposure is rather vague, often debatable and perhaps less valid. Suburban locations are fast becoming urban in character, and a good many tall buildings are being built around airports. It's often impossible to prediet, in view of the surge of building activity which lies ahead, whether, within the next 30 or 40 years, many Type B locations will not become Type A, or even whether a building erected today in a Type A location, surrounded by other tall buildings, may not, during the course of urban renewal, demolition, and the creation of open spaces, eventually find itself in a relatively exposed Type $\mathrm{B}$ location. The architect is concerned with knowing what the maximum intensity of local loads will likely be during the life of the building. For major buildings, these critical loads may be determined in wind tunnel tests, using models which include all structures and other barriers to wind within a radius of several miles. I'm questioning whether these surroundings should be those currently existing and planned for the near future or, instead, should represent that combination of structures which will produce the highest local pressures, both positive and negative, on the building being designed.

Reference has already been made to the fact that failures of windows and other wall elements often do occur during high winds, indicating, without question, some weakness in either design or workmanship. In many cases it's problematical which is at fault. Few, if any failures of this kind are very dramatic. There are no widely publicized classic examples to compare, for instance, with the Tacoma bridge disaster. In fact local failures are usually hushed up, by the building owner, the architect, or both, for reasons that are rather obvious, and seldom do we hear much about them. I can, however, cite a few representative case histories, though I'm not at liberty to identify most of them.

Several years ago, when the wind was gusting to no more than $50 \mathrm{mph}$, several large sheets of plate glass, in windows near the corner, and about midheight of a large new office building in downtown New York City were broken out of their frames by

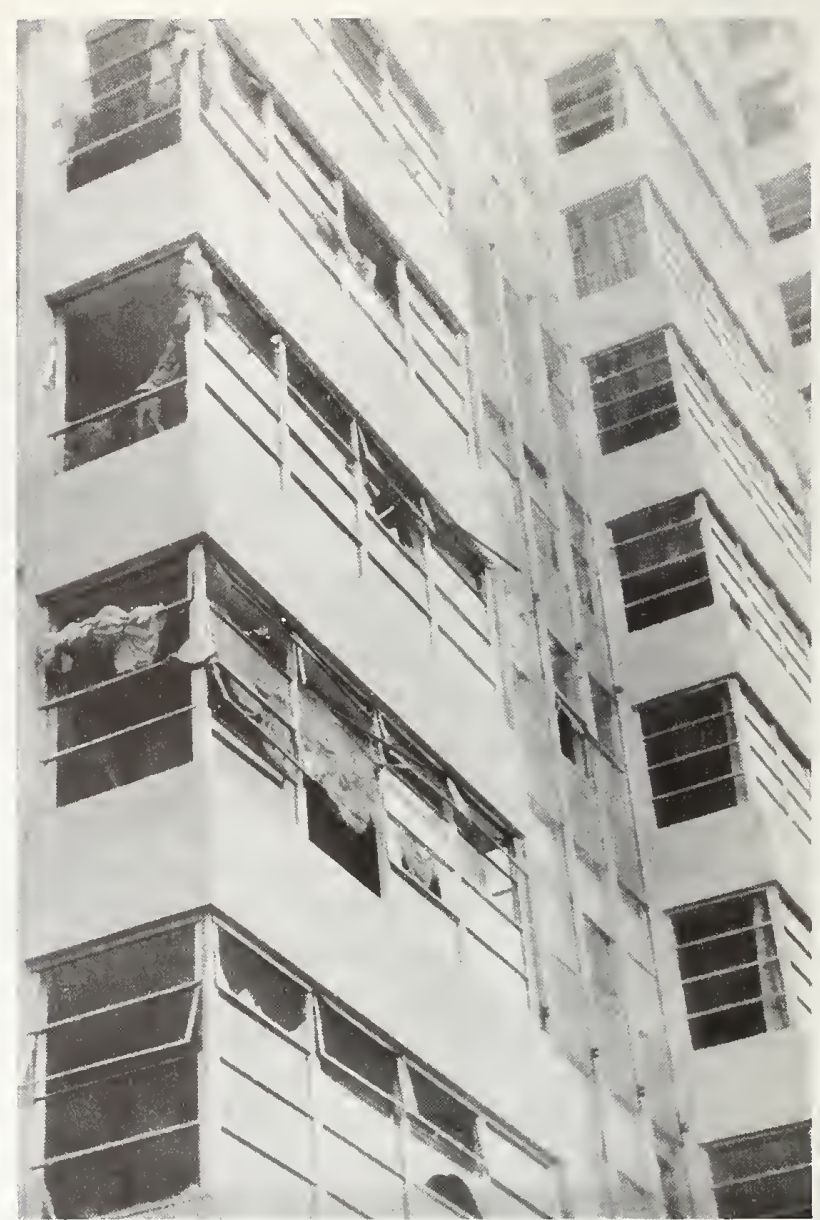

Figure 1. Wind damage to building on Florida Coast, August 1964.

suction forces and fell into a plaza area some $20 \mathrm{or}$ 30 stories below. Fortunately, no one was hurt.

On a gusty day in March 1967, the wind whistled around the corner of another major skyscraper in uptown Manhattan, ripping off a spandrel panel and sucking the glass out of corner winclows on the 18th and 21st floor's.

The third case, illustrated in Figures 1 and 2, involved mueh more extensive damage to a burlding on the Florida coast, during Hurricane Cleo, in August 1964. Several hundred windows were blown out, and many of the window frames themselves were damaged beyond repair. Note that in this instance, too, most of the damage occurred near the corners of the building.

The fourth ease, shown in Figure 3, occurred over 10 years ago, when a severe wind storm hit Philadelphia and blew several wall cladding panels off of the Sheraton Hotel. The architect, commenting on 


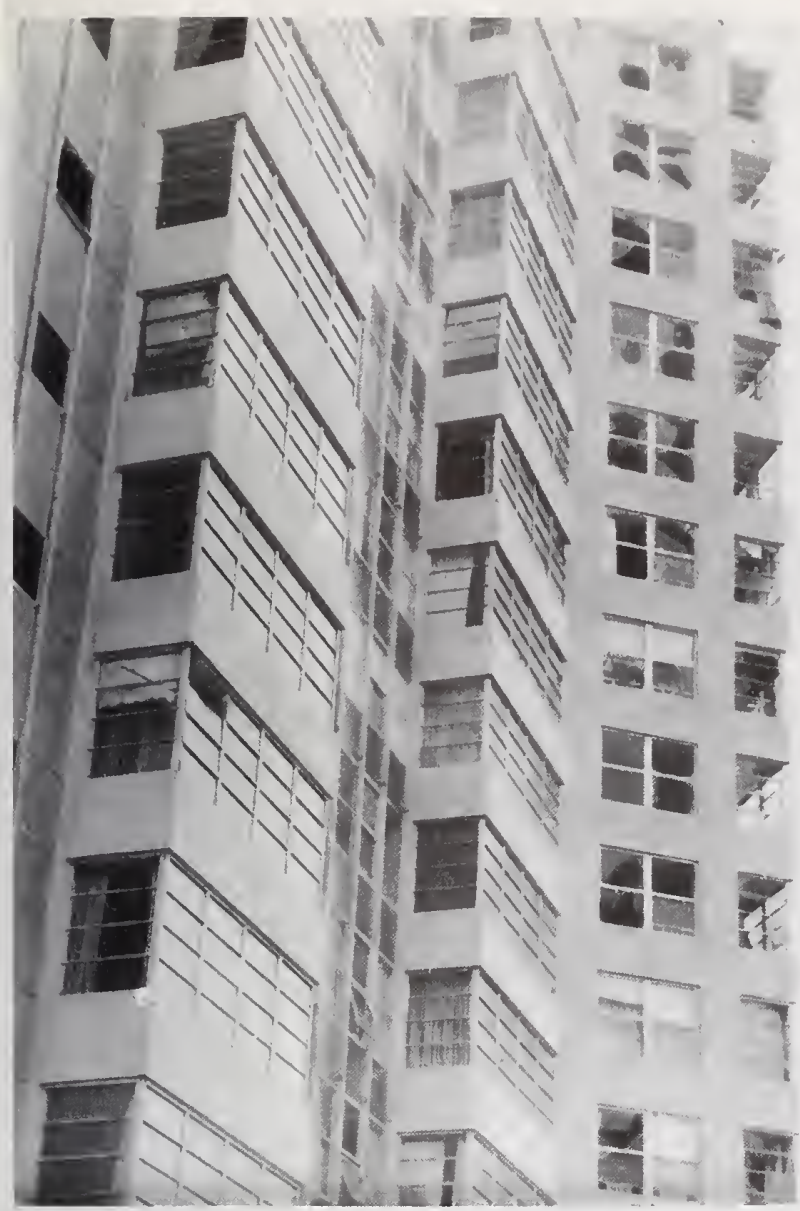

Figure 2. Wind damage to building on Florida Coast, August 1964.

this failure later at a technical meeting [1], reported in part as follows: "Two bays in from the west corner, at the 6 th and 8 th floors, steel clips holding the mullions of the aluminum grid to the concrete fireproofing failed dramatically. They had been cold formed at a sharp angle, introducing a flaw in the metal, and each sheared off at the bend as clean as if a hacksaw had been used to cut them. This allowed the mullions to bulge outward, the steel panels pulled away from the grid and one flew across the street and punched a hole in a church roof. Others fell on our own roof. Fortunately, no one was hurt. The occupants of the rooms were saved from being blown into the street by the concrete block back-up wall. On investigation, we found that the coldformed steel clips which had failed were Z-shaped, whereas the other clips in the area were U-shaped.

* Figures in brackets indicate literature references at the end of this paper.

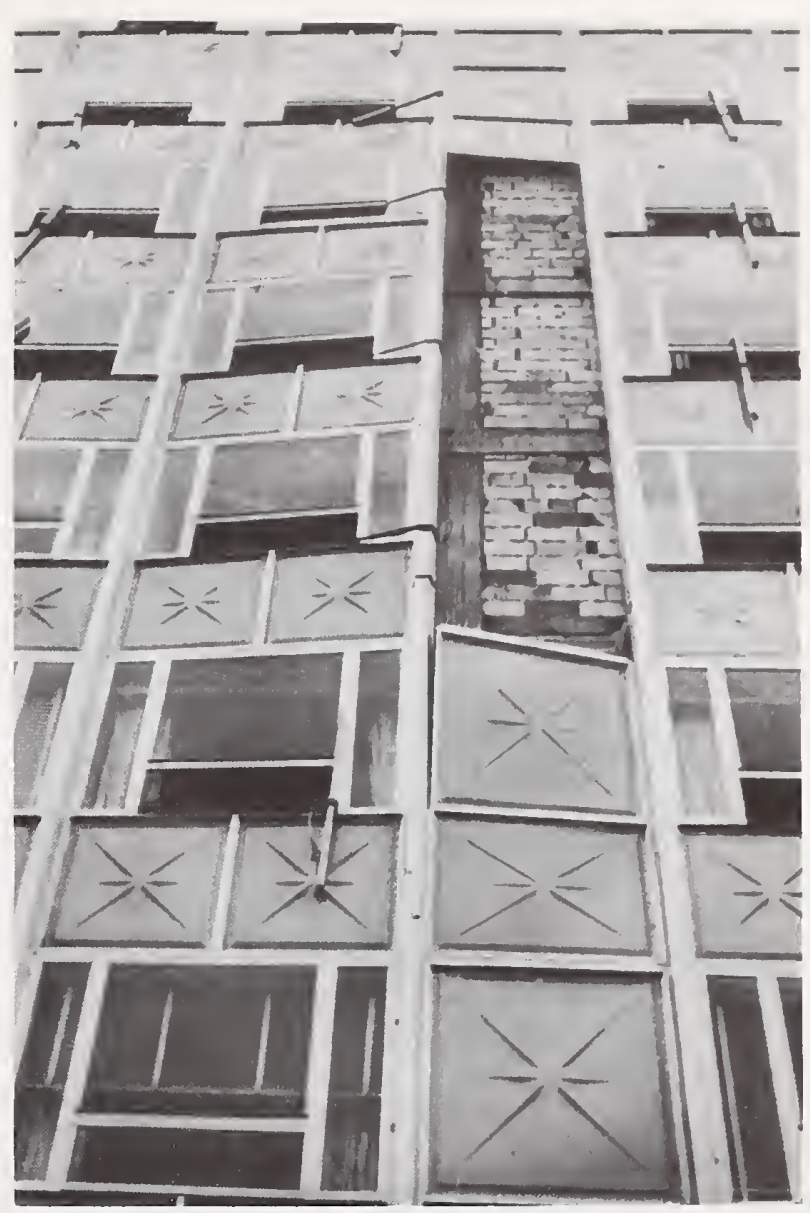

Figure 3. Wind damage to wall of Philadelphia Sheraton Hotel, June 1958.

Only the $\boldsymbol{U}$ clips had been shown on the shop drawings which we harl approved."

These are only four examples, but I'm sure that with diligent searching many more could be found. It's significant, I think, that in all of these cases, just as in others you may recall, the failures were completely local in character. In no case was there any damage, or even any indication of danger, to the structure as a whole.

Another aspect of local wind effects which is also of concern to knowledgeable architects, rather than to the engineer, is the funneling effect often experienced near the base of buildings. Many of you, I'm sure, have learned from experience why Chicago is called the "windy city." It's not only because it's located on a lakefront, but because of the surprising wind velocities that occur around the bases of many of its tall buildings. You may have experienced similar aggravations in the canyons of lower Manhattan. 


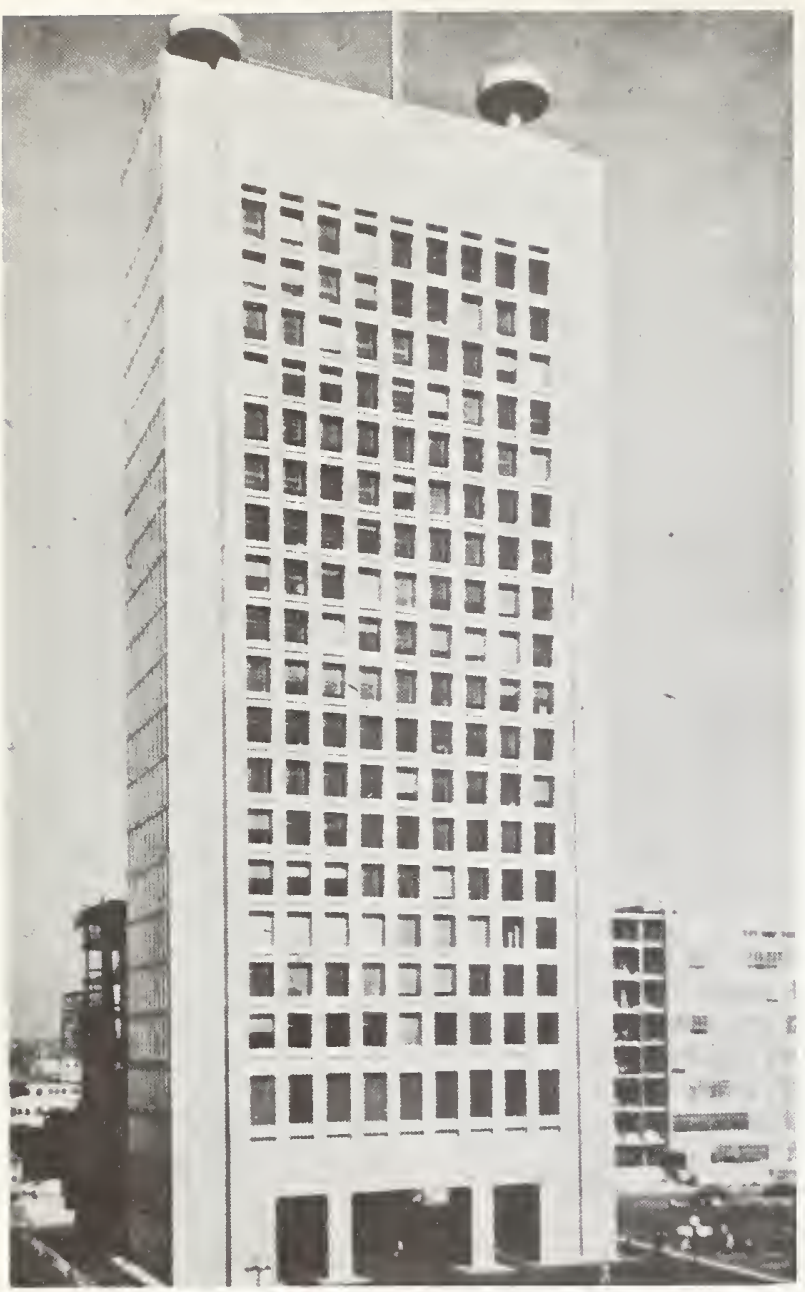

Figure 4, Earth Sciences Building, M.I.T

One building, in particular, where this funneling effect has been a serious problem, is, rather surprisingly, a building on the M.I.T. campus in Cambridge, Massachusetts. This building is the Earth Sciences Building (Figure 4), a $277 \mathrm{ft}$ high tower-like structure which is pierced at the ground level by an arcade $70 \mathrm{ft}$ wide by $21 \frac{1}{2} \mathrm{ft}$ high, where small cyclones are experienced frequently. Simultaneous anemometer readings on this building, on one occasion, showed wind velocities in the 80 to 90 mph range through this arcade, while velocities at the roof level were only 40 to $50 \mathrm{mph}$. Localized wind velocities in the area have been a hazard to pedestrians, and a substantial part of the university's physical plant maintenance fund has been spent on just keeping hardware on the entrance doors opening on to this arcade. To remedy the situation, a temporary plywood screen wall was erected, but of course this was an architectural eyesore.

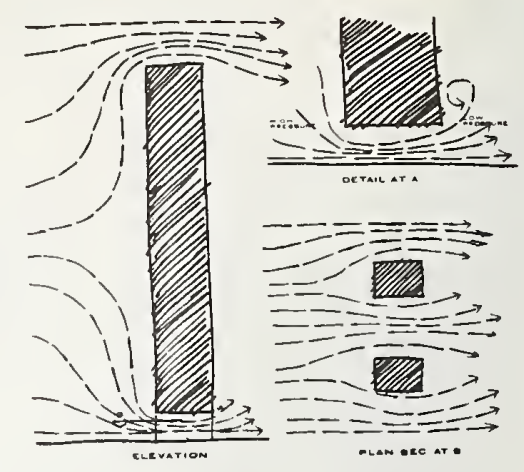

Figure 5. Diagram of wind flow, Earth Sciences Building. Source: Michael O'Hare.

Michale O'Hare, while still a graduate student at Harvard, made a detailed study of this phenomenon, using model and wind tunnel techniques [2, 3] and his findings were both interesting and informative. He also studied similar problems occurring in the plaza of Boston's Prudential Center, where it was found necessary to erect a series of permanent plate glass wind screens to keep pedestrians from literally being blown into the reflecting pool. The O'Hare studies showed that, as night be expected, in both of these cases, the disposition of the surrounding buildings, as well as the downward wind flow on the face of the tall building (Figure 5) both contribute to these high local wind velocities.

It's unlikely that provisions for unique wind effects such as funneling and venturi action can be made in codes or standards. These are phenomena which result from the nature and arrangement of masses affecting wind flow, and each situation requires its own analysis. The architect must be aware of their possibilities, however, in the planning stage, should be able to recognize potential problems, and should know how to cope with them.

Codes or standards should, however, provide information as to the amount and character of loading to which wall elements of typical buildings will likely be subjected. At present we have only one standard, as far as I know, which does this. This is the Tentative Standard for Design Wind Loads for Walls of Rectangular Buildings, published in 1967 by the National Association of Architectural Metal Manufacturers and designated as NAAMM Standard WL-10-67. This standard was developed over a period of about $2 \frac{1}{2}$ years, by a committee composed not only of representatives of several nember companies of NAAMM, but also representatives of ASA Committee A58, the National 
Research Council of Canada, the U.S. Environmental Data Service and other interestcd bodies, who served as advisors. The work was prompted chiefly by two considerations: the recognized need for information about wind loads on wall surfaces, and the acknowledged fact that the data on which ASA Standard A58.1-1955 was based had become obsolete. The intent, in providing this standard was not, in any sense, to question or compete with the work of Committee A58 of the then ASA, now USASI. Quite the contrary, in fact. The purpose was to supplement the work of that Committee, translating it into specific requirements for wall design. Members of Committee A58 actively assisted in the work, and every effort was made to reflect in the NAAMM Standard the same philosophies on which the long-awaited and much-needed USASI Standard was expected to be based.

NAAMM Standard WL-10-67, as stated in its foreword, recognizes that "appropriate wind loads to be used in the design of building walls and their component parts depend upon several variable factors: (1) the geographic location of the building, (2) the degree of exposure or protection provided by its surroundings, (3) the shape of the building,
(4) the height of the building, and in some cases, (5) the location on the building facade of the wall area in question." Accordingly, it includes the latest wind velocity map for the country, provided by the Environmental Data Service, and tabulates recommended design loads for various base velocities and various heights for cach of three types of terrain or exposurc. Each of these exposures, Type A, Type B and Type $\mathrm{C}$, is defined as I have previously indicated.

Figure 6 shows the table of design loads recommended for Type A exposure. The $K_{h}$ values in the second column are pressure cocfficients, which increase with the height $h ; T_{h}$, in the third column, is an exposure factor, decreasing with height, which is used in deriving the gust factor-or more correctly, the gust response factor, shown in the fourth column. The $K_{h}$ and $T_{h}$ valucs were provided by Mr. Vellozzi, in his work on behalf of Committee A58, and this work has since been the subject of an ASCE publication [4]. These values, I believe, are based on Professor Davenport's exposure categories and the power laws governing wind velocity profiles. The so-called gust factor values listed in the fourth column are equal, in the case of relatively small areas, to $0.65+4 T_{h}$.

\section{TABLE A DESIGN WIND PRESSURES (PSf) FOR TYPE A EXPOSURE}

\begin{tabular}{|c|c|c|c|c|c|c|c|c|c|}
\hline \multirow[b]{2}{*}{$\begin{array}{l}\text { Height } \\
\text { (feet) }\end{array}$} & \multirow[b]{2}{*}{$\mathbf{K}_{\mathbf{h}}$} & \multirow[b]{2}{*}{$T_{h}$} & \multirow[b]{2}{*}{$\begin{array}{l}\text { Gust } \\
\text { Factor }\end{array}$} & \multicolumn{6}{|c|}{ Wind Velocities and ( $\mathrm{q}_{30}$ values) } \\
\hline & & & & $\begin{array}{c}60 \\
(9.2)\end{array}$ & $\begin{array}{c}70 \\
(12.5)\end{array}$ & $\begin{array}{c}80 \\
(16.4)\end{array}$ & $\begin{array}{c}90 \\
(20.7)\end{array}$ & $\begin{array}{c}100 \\
(25.6)\end{array}$ & 100 \\
\hline 10 & 0.2 & .37 & 2.13 & $15^{*}$ & 15 & 15 & 15 & 15 & \\
\hline 20 & 0.2 & .37 & 2.13 & 15 & 15 & 15 & 15 & 15 & \\
\hline 30 & 0.2 & .37 & 2.13 & 15 & 15 & 15 & 15 & 15 & \\
\hline 40 & 0.24 & .337 & 2.00 & 15 & 15 & 15 & 15 & 15 & \\
\hline 50 & 0.28 & .315 & 1.91 & 15 & 15 & 15 & 15 & 15 & \\
\hline 100 & 0.44 & .253 & 1.66 & 15 & 15 & 15 & 17 & 21 & \\
\hline 200 & 0.69 & .201 & 1.45 & 15 & 15 & 18 & 23 & 28 & \\
\hline 300 & 0.90 & .175 & 1.35 & 15 & $\overline{17}$ & 22 & 28 & 34 & \\
\hline 400 & 1.08 & .160 & 1.29 & 15 & 19 & 25 & 32 & 39 & \\
\hline 500 & 1.27 & .149 & 1.25 & 16 & 22 & 29 & 36 & 45 & \\
\hline 600 & 1.43 & .140 & 1.21 & 18 & 24 & 31 & 39 & 49 & \\
\hline 800 & 1.73 & .127 & 1.16 & 20 & 28 & 36 & 46 & 57 & \\
\hline 1000 & 1.98 & .117 & 1.12 & 22 & 30 & 40 & 50 & 62 & \\
\hline \multicolumn{4}{|c|}{ over 1000} & \multicolumn{6}{|c|}{$\begin{array}{l}\text { Design load Values for heights and/gr velocities in shaded area should } \\
\text { determined by/special engineering analysis. }\end{array}$} \\
\hline
\end{tabular}

* 15 psf is minimum design load to be used in any case.

Figure 6. Design Wind Loads for Type A Exposure, NAAMM Standard WL-10-67. 


\begin{tabular}{|c|c|c|c|c|c|c|c|c|c|}
\hline \multirow[b]{2}{*}{$\begin{array}{l}\text { Height } \\
\text { (feet) }\end{array}$} & \multirow[b]{2}{*}{$\mathrm{K}_{\mathrm{h}}$} & \multirow[b]{2}{*}{$\mathrm{T}_{\mathrm{h}}$} & \multirow[b]{2}{*}{$\begin{array}{l}\text { Gust } \\
\text { Factor }\end{array}$} & \multicolumn{6}{|c|}{ Wind Velocities and ( $\mathrm{q}_{30}$ values) } \\
\hline & & & & $\begin{array}{c}60 \\
(9.2)\end{array}$ & $\begin{array}{c}70 \\
(12.5)\end{array}$ & $\begin{array}{c}80 \\
(16.4)\end{array}$ & $\begin{array}{c}90 \\
(20.7)\end{array}$ & $\begin{array}{c}100 \\
(25.6)\end{array}$ & Ove \\
\hline 10 & 1.0 & .167 & 1.32 & $15^{*}$ & 18 & 24 & 30 & 37 & \\
\hline 20 & 1.0 & .167 & 1.32 & 15 & 18 & 24 & 30 & 37 & \\
\hline 30 & 1.0 & .167 & 1.32 & 15 & 18 & 24 & 30 & 37 & \\
\hline 40 & 1.07 & .160 & 1.29 & 15 & 19 & 25 & 31 & 39 & \\
\hline 50 & 1.15 & .154 & 1.27 & 15 & 20 & 26 & 33 & 41 & \\
\hline 100 & 1.40 & .140 & 1.21 & 17 & 23 & 31 & 39 & 48 & \\
\hline 200 & 1.70 & .125 & 1.15 & 20 & 27 & 35 & 45 & 55 & \\
\hline 300 & 1.90 & .117 & 1.12 & 22 & 29 & 38 & 49 & 60 & \\
\hline 400 & 2.05 & .112 & 1.10 & 23 & 31 & 41 & 51 & 64 & \\
\hline 500 & 2.20 & .108 & 1.08 & 24 & 33 & 43 & 54 & 67 & \\
\hline 600 & 2.31 & .105 & 1.07 & 25 & 34 & 45 & 56 & 70 & \\
\hline 800 & 2.53 & .102 & 1.06 & 27 & 37 & 48 & 61 & 76 & \\
\hline 1000 & 2.60 & .098 & 1.04 & 27 & 37 & 49 & 62 & 77 & \\
\hline \multicolumn{4}{|c|}{ over 1000} & \multicolumn{6}{|c|}{$\begin{array}{l}\text { Design load balues for heights and /or velocities in shaded area should } \\
\text { determined by special engineering analysis. }\end{array}$} \\
\hline
\end{tabular}

*15 psf is minimum design load to be used in any case.

Figure 7. Design wind loads for type C exposure, NAAMM standard WL-10-67.

Note that the minimum load recommended is 15 psf, and that no loads are tabulated for wind velocities exceeding $100 \mathrm{mph}$ or heights exceeding 1,000 feet. Special engineering analysis is recommended for such conditions. All load values listed are to be used by the designer as both positive and negative loads, the two being considered equal.

Figure 7 shows the corresponding table for design loads to be used with Type $\mathrm{C}$ exposure. Of course there is also a similar table for Type B exposure, the values being intermediate between those for Type $\mathrm{A}$ and Type $\mathrm{C}$ exposure. The standard also includes a graph of the full range of $K_{h}$ and $T_{h}$ values, to facilitate determining intermediate values not listed on the tables.

This NAAMM Standard has been rather widely recognized and commended since its publication about two years ago and, I think, is serving a useful purpose. It should be emphasized, though, that it's intended as a tentative, being subject to revision as newly developed information may dictate.

Before concluding, I'd like to refer very briefly to several examples of recent and current work contributing to our information about critical local wind loads. As you know, our colleagues in Canada have been engaged for some time now, in recording wind pressure measurements on several high-rise buildings, and I'm looking forward to learning more about recent findings in that work later in this meeting. You are aware, too, that similar important field work of this nature is being done by the Building Research Station in England. The drawings of Figure 8, for example, from the report by Newberry,

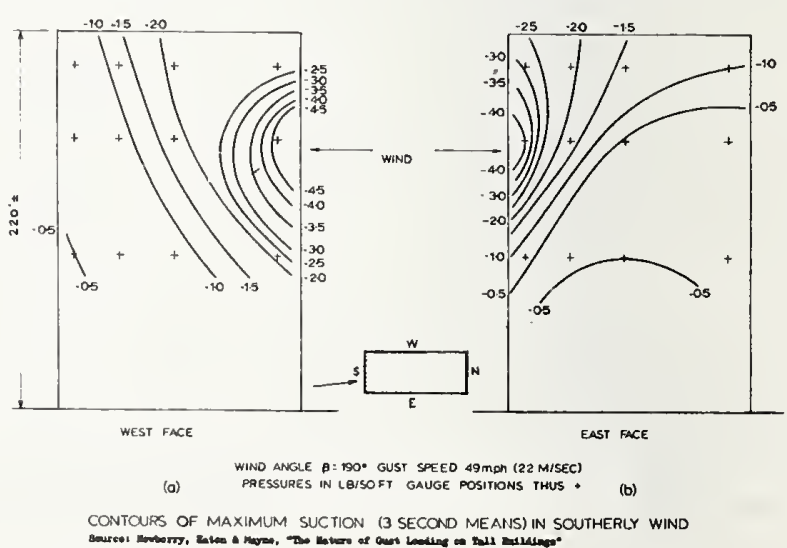

Figure 8. Measured wind pressures on walls of Royex Building, London. Source: Newberry, Eaton \& Mayne. 

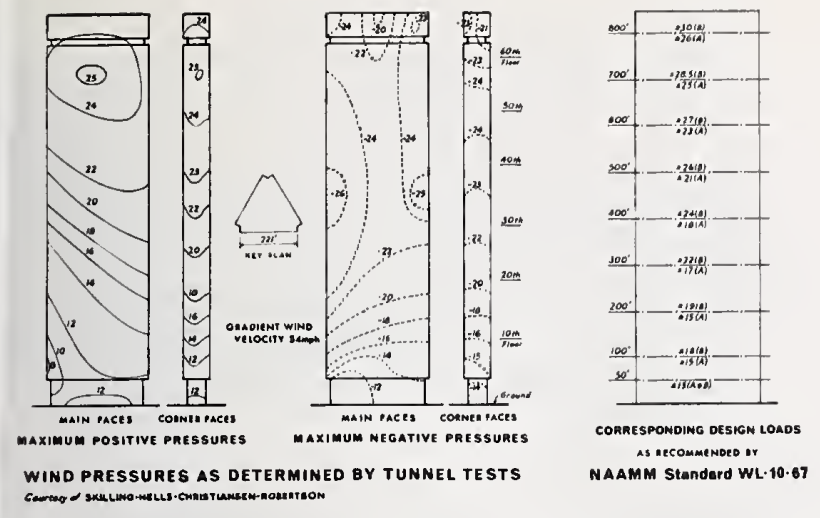

Figure 9. Wind loads on walls of U.S. Steel Building, Pittsburgh.

Eaton and Mayne [5], show the pattern of loads determined by measurements on the Royex Building in London several years ago. Boundary layer wind tunnel tests with models are also becoming more and more important, if not indispensible, as an aid to both engineers and arehiteets in the design of major high-rise buildings. The two lefthand drawings in Figure 9, for which I'm indebted to the firm of Skilling, Helle, Christensen, Robertson, show, for example, the distribution and intensity of wind loads, as determined by wind tunnel tests, on the walls of the new U.S. Steel building now under construction in Pittsburgh. For purposes of comparison, I have taken the liberty of including at the right a cliagram at the same scale, indicating the comparable wind loads prescribed by the NAAMM Standard. Loads for both Type A and Type B exposure are shown, $A$ below the lines and $B$ aloove, as this is one of those cases I referred to earlier, where proper elassification as to exposure scems questionable. There appear to be no glaring discrepancies between the predicted and the prescribed loads as to their magnitude, but the distribution pattern of loading eonsistently shown by model test, as well as by field measurements, indicates that we are likely in error in assuming, as all codes and standards currently do, that loads increase consistently with building height, with the maximum load oceurring at the very top of the building.

One more item of eurrent interest eoncerns the local wind loads being used for the walls of the new World Trade Center buildings in New York, which, as you probably know, will be the tallest buildings in the world. I am informed by a reliable souree that the loads specified for the testing of these walls are as follows: positive loads which range from 45 psf for the lower 41 stories to 55 psf for the next
67 stories, but are reduced to $40 \mathrm{psf}$ for the top 3 stories; negative loads which are nuch higher, ranging from $65 \mathrm{psf}$ for the lower 6 stories to 125 psf for the 101st through 109th stories, with four intermediate increments, and are $115 \mathrm{psf}$ for the top story.

I made reference at the outset to information gaps, and to me this is a matter of major concern. In many areas of building technology the time lag between the aequirement of information through research, and the dissemination of this knowledge for gencral use is far too great. This is particularly true in respect to design wind loads. The eollective knowledge in this field, represented in this room today, is tremendous. But it can be of small benefit to the great majority of practitioners until it is translated into practical working data for the designer's use. This process should be hastened. Although, some problems may still remain unsolved, and certain theories may not yet be fully verified, an organized effort should be made to see that codes and standards more promptly reflect current knowledge. There should be some central elearing house which would circulate eurrent technical information, in this field as well as other's, at frequent intervals to all concerned. If the Bureau of Standards could find a means of doing this, it would be providing a great service to the building industry.

To summarize, then, the more important concerns with respect to the influenee of wind on the design of wall elements are these:

1. Local wind loads acting on the walls of a building are not the same as the overall loads governing the design of its structure; usually their intensity is greater.

2. The maximum negative loads aeting on wall areas are usually as large as, if not greater than, the maximum positive loads, and may be several times greater.

3. The maximum loads on the faces of tall reetangular buildings probably do not occur at the top of the wall, but at some distance below the top.

4. In planning the arrangement of building units within a group, and the relation of roids to mass at the ground level, the possible creation of wind funneling should be carefully eonsidered.

5. Accumulated knowlerlge regarding wind loads should be more widely and more promptly disseminated to the design professions. 
6. Without question there is need for much more information regarding local wind effects, if designers of building walls are to steer a true course between the creation of hazardous conditions on the one hand and wasteful overdesign on the other.

\section{References}

[1] Dean, Robert C.: "Sheraton Hotel, Philadelphia," Publication 788 National Academy of Sciences-National Research Council (1960).
[2] O'Hare, Michael: "Wind Whistles Through M.I.T. Tower," Progressive Architecture, March 1967.

[3] O'Hare, Michael: "Designing with Wind Tunnels," The Architectural Forum, April 1968.

[4] Vellozzi, J. and Cohen, E.: "Gust Response Factors," Paper 5980, Proceedings of the American Society of Civil Enginecrs, Vol. 94, No. ST6, June 1968.

[5] Newberry, C. W., Eaton, K. J. and Mayne, J. R.: "The Nature of Gust Loading on Tall Buildings," Paper No. 15, Proceedings of the International Research Scminar, National Research Council, Ottawa, Canada, Septcmber 1967, Vol. 1. 
SESSION 2.

\section{A'TMOSPHERIC CONSIDERATIONS}

Chairman: Mr. H. C. S. Thon

Senior Research Fellow

Environmental Data Service-ESSA

Silver Spring, Md. 20910 



\title{
GUIDELINES IN SELECTING WIND MEASURING INSTRUMEN'TS AND 'TIEIR LOCATIONS FOR WIND LOADING STUDIES*
}

\author{
Gerald C. Gill \\ Professor of Meteorology \\ University of Michigan \\ Ann Arbor, Michigan 48104
}

\begin{abstract}
The different types of wind speed sensors (cup, propeller, pressure tube, pressure sphere, hot, wire, and sonie) will be discussed in relation to their suitability for wind loading studies. For very sound reasons the rotational types of anemometers (cup and propeller) have become the standard wind speed sensors used in North America today. Pressure type wind speed sensors will be eonsidered but they have inherent weaknesses as anemometers. In view of the versatility of the rotational anemometers and in view of all weather records and charts being based on observations with these instruments it appears advisable for wind loading studies to measure wind speed with these conventional anemometers.

Wind vanes of different types (flat plate, splayed vane, and aerodynamic cross section) will be discussed and the transducers to be used with them. Very light vanes of the flat plate type are recommended.

The dynamic response and fidelity of recording of both anemometes and wind vanes will be discussed.

The wind-shadow effect of towers on the wind sensors mounted thereon, and the orientation of the sensors will be discussed. For good exposure the sensors should be mounted one to two tower widths out from the tower, and, either into the prevailing wind, or, into the wind direction of maximum concern. The height and location of towers relative to the building or structure under study will be discussed.
\end{abstract}

Key words: Anemometers; instrumentation; tower interference; transducers; wind vanes; wind loads.

* Note.-Due to illness, the author was not able to complete the manuscript in time for publieation. 



\title{
THE ADEQUACY OF EXISTING METEOROLOGICAL IDA'TA FOR LVALUA'TING; STRUCTURAL PROBLEMS
}

\author{
Irving A. Singer and Maynard E. Smith \\ Brookhaven National Laboratory \\ Upton, Long Island, New York 11973
}

\begin{abstract}
Available meteorological data are usually unsuitable for application to structural problems for a variety of reasons. The most obvious deficiency involves instrument location in the threedimensional sense. Most meteorological observations have been obtained at low devation in open country, and they therefore do not reveal the wind structure at the typical heights of modern urban buildings. Furthermore, few of the data have been processed to reflect the turbulent structure of the flow. More subtle problems are involved with the failure of any current programs to define the horizontal structure of turbulence, although a small number of appropriate studies have been made in the vertical. Very important also is the lack of data taken under extreme meteorological conditions, such as thunderstorms and hurricanes. These deficicneies derive in part from the difference in objectives that have led to existing metcorological programs and those that would suit the structural engineer, but they are also associated with the failure of the latter to define his requirements. An experimental program that may fill some of the gaps is outlined for discussion and criticism.
\end{abstract}

Key words: Climatology; meteorology; spatial correlation; turbulence; wind observations; wind profiles.

\section{Introduction}

Ritter and Ordway, in a presentation before the New York Academy of Sciences [1]* noted that: "It is a peculiar fact that until now climate has been a minor factor in the design of our current urban renewal programs," and they cite a variety of problems that may be created by, and conversely influence, the construction of massive buildings of new and unusual configurations. It is true that there has been surprisingly little conversation among the aerodynamicists, architects, structural enginecrs and meteorologists, and it is imperative that such exchanges begin. In our own limited probing into this field, we have found that a need exists for a review of the available wind data that can be applied to such problems, and this paper includes such a review. Since our consideration of the problem reveals important gaps in the meteorological data, suggestions for remedying the deficiencies are also included.

\section{Standard ESSA Wind Observations}

By far the most complete wind data available in the United States reside in the records of the U.S. Weather Bureau (ESSA) stations, which number

* Figures in brackets indicate the literature refcrence at the end of this paper. more than 1,000. Many of these stations have been in operation for one or more decades, and ESSA has provided an excellent repository and processing center at Asheville, N.C. so that data can be retrieved and reprocessed readily.

The typical "surface" wind observation is usually obtained from a sluggish cup anemometer and vane assembly, mounted in a well-exposed location about $30 \mathrm{ft}$ above the ground or building structure. The vast majority of the sites are at airports, and urban observations are comparatively rare. The standard observation procedure is to note the indicated wind speed and direction for a brief period once each hour, and record them to $10^{\circ}$ (formerly 16 compass points) and the nearest knot or mile per hour. Normally a contacting device is associated with the anemometer, from which one can obtain the time required for one mile of wind flow to pass the instrument. Observations called PIBALS or RABALS are taken at many of the stations, using either visual or electronic tracking of a rising balloon several times per day to determine the variation of wind with height. These data are processed to reflect the wind at $1,000-\mathrm{ft}$ intervals above mean sea level.

Unfortunately, neither the surface nor upper wind data offers direct and substantial assistance to the structural engineer. One can obtain at best a crude impression of the change of wind with height, and statisties of the distribution of the "hourly" wind 
speeds and directions. It is possible to develop reliable estimates of the maximum winds at anemometer elevation, since the length of record is usually sufficient for the purpose and observations are made during extreme as well as typical meteorological conditions.

Obviously, no indication of short-term wind fluctuations other than the variable period "fastest mile" can be derived from these records, nor can one reach any valuable conclusions about the smallscale spatial distribution of the wind field.

\section{Specialized Data}

Most of our knowledge of the details of wind fluctuations comes from a limited group of tower observations, generally extending no more than 500 $\mathrm{ft}$ above ground, although there are a few reaching $1,000 \mathrm{ft}$ and more.

Unlike the standardized ESSA surface stations, these towers have been equipped with a variety of wind sensors, ranging from hot-wire anemometers to Bendix-Friez Aerovanes, and the recording and processing of the data are almost as varied as the number of towers themselves. Furthermore, and by no means trivial, is the fact that few of these installations have been operated during extreme meteorological conditions. Most of the research associated with these towers has been directed toward typical weather regimes, and equipment is frcquently removed if the probability of damage by lightning or strong winds is anticipated.

From these towers, however, we have obtained our only records of the detailed wind structure that is important in structural design. A fair amount of these data could be reduced to a useful form if this has not been done already. Certainly we are in a position to define typical vertical wind profiles up to $500 \mathrm{ft}$ above ground in open country at a minimum of 20 locations within the United States. At many of these locations spectra or other expressions of time and space correlation covering wavelengths of $10 \mathrm{~m}$ and longer can be developed for the $u$ and $v$ components of the wind, and in rarer instances for the $w$ component. In a few isolated instances, some of these data are available for extreme wind conditions. Spatial representation is confined almost exclusively to a single vertical line, and horizontal correlations are available only at very low elevations.

Above $500 \mathrm{ft}$ information becomes very sparse, both in terms of the number of sites and in the suitability of the data. Most of the information has been processed so that fluctuations having periods shorter than 5 minutes cannot be studied, and in only one case [2] are we aware of complete three-dimensional records up to $1,000 \mathrm{ft}$.

To the best of our knowledge we have no current source of information linking low and high-level fluctuations in a major urban area, nor do we have any indication of the horizontal distribution of wind fluctuations high above the ground, except by blind dependence on some theoretical transformation from time to space scales. The current vogue among meteorologists is Taylor's hypothesis which permits transfer from one coordinate system to another, but this approach has been tested only at low elevations and in the direction of the mean wind. Lack of thrce-dimensional observations extending to higher elevations is the most serious shortcoming of the existing data.

\section{Suggested Remedies}

Despite the foregoing gloomy remarks, there are many constructive steps that could be undertaken, and in which our organization is interested. The first is an intensification of exchanges of which the present meeting is an example. We must determine what meteorological data would be most valuable in analyzing structural problems and the form in which they could be most easily utilized. As meteorologists we suspect, for example, that turbulence measurements on a very finc scale may have no application to these problems, but we are not certain of this conclusion. The choice and location of instrumentation for new studies, however, is strongly dependent upon the time and space scales of interest.

Surely a climatology of mean and maximum velocity profiles can be established from the tower data we now have. These profiles should be developed to cover various averaging periods, meteorological conditions and topography (including cities wherever possible) and they should be expressed in probability statements such as recurrence intervals.

One can also attempt to relate the profile data to the standard ESSA observations. If this effort is successful, the huge body of standard data will increase greatly in value.

A climatology of the good data from existing towers should be made. The adjective "good" implies appropriate instrumentation, recording and processing, so that much of the existing record wouk be eliminated, but even the most meagre climatology, segregater into meteorological, topographic and height classifications should be valuable. 
New data obtained with the objectives of structural design as controlling criteria should obviously be collected, and appointment of an advisory committee for the program including represcntation from the fields of aerodynamies, instrumentation, meteorology, statisties and structural engineering should therefore be the first order of business. We have quite enough experimental data that arc not suitable for such purposes.

We belicve that the most valuable experimental effort should be aimed at determination of the full details of the threc-dimensional wind structure at a relatively simple, rural site. The kcy features of the program should be the utilization of instrumentation of appropriate response, durability and reliability, and the definition of the horizontal spatial correlations in the flow. An array of towers, one of which should reach $1,000 \mathrm{ft}$, would be needed. Several mobile units approximately $150 \mathrm{ft}$ high should be included to determine the horizontal variability adequatcly. Recording equipment must be directly suitable for computer analysis, and it is quite possible that an on-line computer should be assigned to the project to permit immediate analysis of the data and reorientation of inclividual experiments.

An open, uncomplicated site is suggested in preference to an urban installation, since we believe that the complexities of flow at the lower levels in citics will always renain unique functions of the particular locality and may never submit to theoretical analysis. On the contrary, it would seem more likely that correct judgements conceming the design of a new urban structure might better be achieved by wind tunnel reproduction of the profile and turbulence characteristics of the unimpeded flow coupled to scale modeling of the salient urban features.

A word of caution about tunnel modeling does seem in order, however. Logically, careful attention to detail should permit reproduction of many characteristics of the natural wind flow, but one wonders whether it will become possible to reproduce the larger scale (though relatively short period) variations in the $v$ and $w$ components of the natural flow. The wind tunnel provides a fixed mean wind direction, while the atmosphere is under no such restrictions.

Elevated measurements within eities should, however, be used in developing our understanding of urban turbulence, and the program should include study of data from structures within cities and the cstablishment of new measuring arrays as well.
The television industry has generously provirled a number of spires that may be useful in observing wind structure within urban areas, and neasurements on poles or morlest towers above the taller buildings may also be helpful in determining the differenees between urban and country wind structures.

One reasonably simple method of establishing the alterations in natural turbulence that may be induced by the introduction of new structures is by. the use of inflatable buildings. ('onsiderable experience with fixed structures has already been obtained in wind tumnels and some of this evaluation has been duplicated in the field, but the emphasis has been placed on the stresses on the buildings rather than the deformation of atmospheric strueture. Inflatable buildings pernit extension of wind tumnel "before and after" techniques to field experiments.

An important eriterion also is that the site of the field experiments be subject to extreme wind conditions with reasonable frequency. Towers such as those in Philadelphia, Cedar Hill and Savannah River, for example, are too far inland to experience maximum hurricane winds, but they certainly will be more frequently affected by squall lines and thunderstorms than coastal sites.

New field experimentation obviously implies more than a single site if it is to be comprehensive and effective. The desirability of duplicating certain measurements at different sites is already clear. The standard deviations of the $u$, and $v$ and $w$ components observed at the 500-1,000 ft levels on the Savannah River tower, for example, are not in agreement with the values that would be derived from theories that seem to describe lower-level measurements, but no one can say whether these data are incorrect, whether the site is somehow anomalous or whether the theory is defeetive.

Such a major program to remedy the defects in our knowledge of turbulence would obviously be expensive, but it would secm that the savings that might accrue in more intelligent structural codes and designs woukd greatly outwoigh the cost.

\section{References}

(1) Ritter, A. and Ordway, O. E.: "The Renaissance of Subsonic Acrodynamies," Annals, X.T. Acad. of Seiences V15t, Art. 2, pp. 777-791, Nov. 196s.

[2| Cooper, R. W. and Rusche, B. C.: The SIRI Meteorological Systems, Proc., US.AEC Met. Into. Mter, sept. 11-1t, 1967, Chalk River, Can., AlCL-27s7, pp. 51-5s. 



\title{
THE CHARACTERISTICS OF ATMOSPHERIC TURBULENCE AS RELA'TED 'TO WIND LOADS ON TALL STRUCTURES
}

\author{
George H. Fichtl, ${ }^{1}$ John W. Kaufman, ${ }^{2}$ and William W. Vaughan ${ }^{3}$ \\ Aerospace Environment Division \\ Aero-Astrodynamics Laboratory \\ NASA-Marshall Space Flight Center \\ Huntsville, Alabama 35812
}

\begin{abstract}
An engineering boundary layer wind model based upon data collected at the NASA 150-meter meteorological tower facility at the Kennedy Space Center, Florida is discussed. A statistical power-law peak wind profile is used to extrapolate peak wind statisties valid at the 10-meter to other levels. The relationship between the instantaneous extreme wind profile and the peak wind profile for various periods of exposure up to ten minutes is examined. The gust factor profilc, which depends on the peak wind speed, height, and averaging time, is applied to the peak wind profile to obtain a mean wind profile. A spectral model of the longitudinal and lateral components of turbulence for the neutral boundary layer (high wind speeds) is presented.
\end{abstract}

Key words: Atmospheric boundary layer; gust factors; peak values; power spectra; turbulence; wind profiles.

\section{Nomenclature}

$b \quad=$ parameter that characterizes the statistics of $k$

$C_{p} \quad=$ specific heat of dry air at constant pressure

$C \quad=$ empirically determined parameter that occurs in formulae of the longitudinal and lateral spectra

$f=n z / \bar{u}$

$f_{m} \quad=$ value of $f$ associated with peak of logarithmic spectrum

$g \quad=$ accelcration of gravity

$g_{0}=$ empirical function of $u_{18}$ and $t$ that occurs in the formula for the gust factor

$G \quad=$ gust factor

$k \quad=$ peak wind profile parameter

$k_{1} \quad=0.4=$ von Karman's constant

$L^{\prime} \quad=$ stability length

$n$ = frequency, $\mathrm{Hz}$

$p \quad=$ empirical function of $u_{18}$ that occurs in the formula for the gust factor

$=$ empirically determined parameter that occurs in formulae of the longitudinal and lateral spectra

$R i \quad=$ gradient Richardson number

$S(n)=$ longitudinal or lateral spectrum of turbulence $S_{2}(n)=$ spectrum of the square of the longitudinal or lateral components of the wind

2 Aerospace Engineel.

2 Chief, Atmospheric Dynamies Branch.

${ }^{3}$ Chief, Aerospace Environment Division. $t \quad=$ averaging time

$\tilde{T} \quad=$ time average mean temperature

$u(z)=$ peak wind speed at height $z$

$\bar{u}(z)=$ time averaged mean wind speed at height $z$

$u * \quad=$ surface friction velocity

$z_{0} \quad=$ surface roughness length

$\beta \quad=$ vertical collapsing factor

$\sigma \quad=$ variance of $k$ or variance of the longitudinal or lateral components of turbulence

$\psi \quad=$ logarithmic wind profile stability defect.

\section{Introduction}

The purpose of environmental wind criteria is to provide a model of the atmospheric boundary layer such that when it is used in design studies an acceptable structure results. How one should develop such a model is determined by the purpose and requirements of the structure. Thus, to develop these models so that they are meaningful from an enginecring viewpoint, it is necessary that the atmospheric scientist and the design engineer work as a team.

The National Aeronautics and Space Administration, Marshall Space Flight Center (MSFC), Huntsville, Alabama, is concerned with developing and operating launch vehicles like the Saturn $\mathrm{V}$, which placed three American astronauts into lunar orbit on December 25, 1968. The Saturn I stands 363 feet tall and weighs over 6 million pounds. Its height makes the vehicle especially vulnerable to ground wind loads. An artist's concept of this 


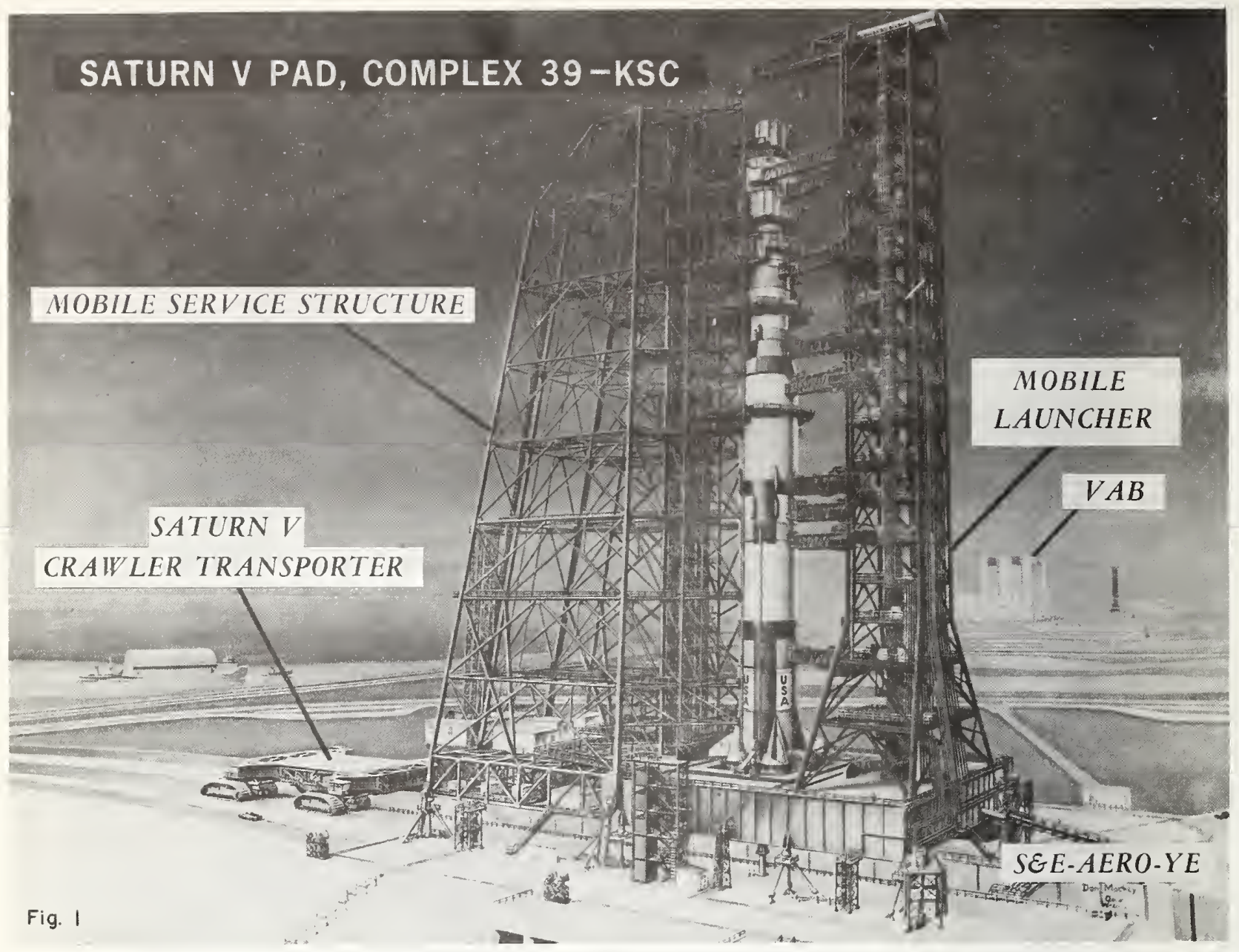

Figure 1. An artist's concept of the Saturn V space vehicle and the associated ground support equipment.

vehicle and its associated ground support equipment is shown in Figure 1. About 2 months before launch, the vehicle is erected in the Vertical Assenthly Building (see background of Figure 1) on the Mobile Launcher. The vehicle and launcher are then moved to the launch parl, about 3 miles away, on the Crawler Transporter. The Mobile Service Strueture is then moved to the vehicle to provide a platform from which the vehicle ean be serviced. Before launch, the Mobile Service Structure is rolled off the parl, and the volicle is launched from the Mobile Launcher:

During the design and fabrication stages of space vehicles, the design engineer must know the final weight of the launch configuration so that unnecessary weight penalties are avoided. Since the ground support equipment will remain on the ground at launch, the present design philosophy in the in- dustry is to design the auxiliary ground support equipment to alleviate, wherever practical, some of the loads on the vehicte due to the ground winds. However, it is conceivable that ground support equipment may have to be so sophisticated that providing this capability will become impractical. Thus, it is imperative that the ground wind environmont be defined in usable engineering terms as preciscly as possible so that the ongineer need not overdesign the ground support equipment and the vehicle strueture for a given value of risk. This is especially true if the vehicle is required to withstand ground wind loarls in the event the ground support equipment cannot be designed to completely alleviate the entire design ground wind loadings. Although there are various ways of presenting wind criteria for enginerring design, this paper describes only the principal methods used at the Marshall Space Flight Center: 


\section{Peak Wind Statistics}

The fundamental surface wind statistics for the Kennedy Space Center are based on an 8-year sample of hourly peak wind speeds measured at the 10-meter level for a period of record from September 1958 through June 1967. The sample was constructed for NASA at the National Weather Records Center, Asheville, North Carolina, by selecting the peak wind speed that occurred in each hour of record read from original wind rolls. Pcak wind statistics have three advantages over mean winci statistics. First, peak wind statistics do not depend on an averaging operation as do mean wind statistics. Second, to construct a sample of mean winds, a chart reader or weather observer must perform an "eye-ball" average of the wind data, thus, causing the averaging process to vary from day to day according to the mood of the observer and from observer to observer. Hourly peak wind speed readings avoid this subjective averaging process because "a peak is a peak is a peak." Third, to monitor winds during the countdown phase of a vehicle launch, it is easier and more objective to monitor the peak wind speed than the mean wind speed.

Smith et al. [1]* have performed extensive statistical analyses with the Kennedy Space Center peak wind speed sample. In the course of his work at the Marshall Space Flight Center, he has introduced the concept of exposure period probabilities into the design and operation of space vehicles. By determining the distribution functions of peak wind speeds for various periods of exposure (hour, day, month, year, etc.), it is possible to determine the probability that a certain peak wind-speed magnitude will occur during a prescribed period of exposure of a space vehicle to the natural environment. Thus, for example, if an operation requires, say, one hour to complete, and if the critical wind loads on the vehicle can be defined in terms of the peak wind speed, then it is the probability of occurrence of the peak wind speed during a 1-hour period that gives a measure of the probable risk of the occurrence of structural failure. Similarly, if an operation requires one day to complete, then it is the probability of occurrence of the peak wind during a 1-day period that gives a measure of the probable risk of structural failure.

Smith et al. [1] have also shown that the peak wind speeds at Cape Kennedy for various periods of exposure have a Fisher-Tippett Type I distribution

* Figures in brackets indicate literature references at the end of this paper.
[2], which is the one used by Gumbel [3]. Althougl the Gumbel distribution appears to give a good theoretical fit to the empirical peak wind speed distribution, it has the disadvantage that it is unbounded at both ends. Since wind speed has a physical lower bound at zero, it may be desirable to investigate other distribution functions. Snith points out that the Fisher-Tippett Type II distribution, which is indeed bounded from below at zero, would be such a function. Thom [4] has used the Fisher-Tippett Type II distribution for representing ground wind statisties.

Figure 2 shows an example of Sinith's peak wind spced statistics where the distributions for the month of October for different reference periods are illustrated, and the probabilities of the occurrences of peak wind speeds for the indicated reference periods can be determined. Thus, for example, the probability that the peak wind speed during the hour from 0530 to 0630 EST will be less than 32 knots is 0.977 .

Probability statements concerning the capabilities of the space vehicles developed at MSFC and launched at the Kennedy Space Center (KSC) are given in terns of Smith's peak wind speed exposure statistics. The statistics are valid at the 10 -incter level. However, to perform loading and response calculations resulting from steady-state and random turbulent drag loads and von Karman vortex shedding loads, the engineer requires information about the vertical variation of the mean wind speed and the structure of turbulence in the atmospheric boundary layer. The philosophy at MSFC is to extrapolate the peak wind statistics up into the

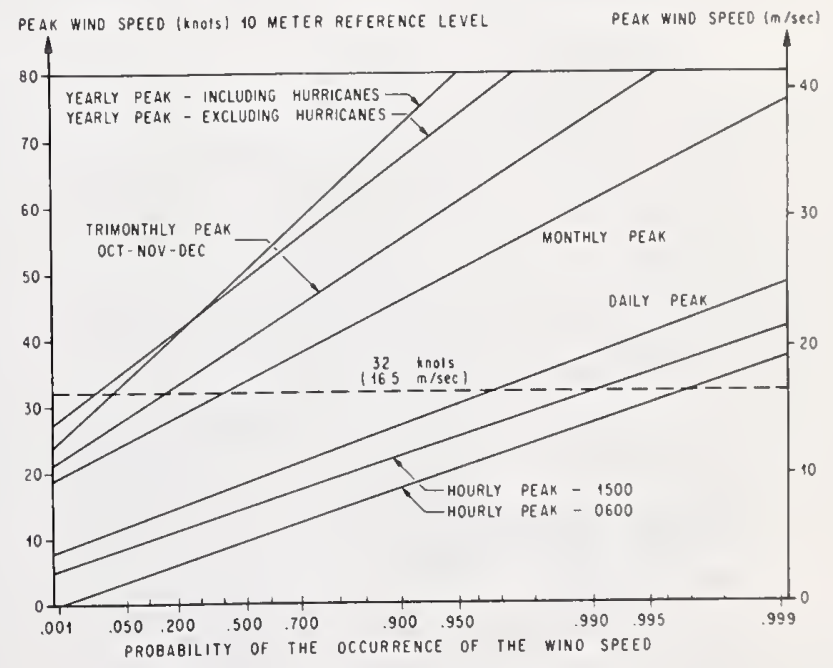

Figure: 2. Fisher-Tippett distribution collated to peak wind speed samples at Cape Kennedy, Florida, in Oetober. 
atmosphere using a peak wind profile, and to obtain the associated quasi-steady or mean wind speed profile by applying a gust factor, which is a function of wind speed and height. At this point, the engineer can calculate the steady-state loads resulting from the mean wind profile and the response due to discrete gusts in the form of a gust factor. The gust factor accounts for the loads beyond those resulting from the quasi-steady wind profile-in short, the turbulence. For some applications, depending on the response characteristics of the vehicle, the peak wind speed profile is used directly in the calculations. An alternative, and probably more meaningful, representation of the turbulence can be given in the form of a spectral model of the longitudinal and lateral components of turbulence.

\section{The NASA 150-Meter Meteorological Tower}

To obtain micrometeorological data representative of the Cape Kennedy area, especially in the vicinity of the Apollo/Saturn V launch pads, a 150-meter meteorological tower was constructed on Merritt Island at the Kennedy Space Center. The tower facility, discussed in detail in a report by Kaufman and Keene [5], is only briefly described here.

\subsection{Terrain Features}

Figure 3 shows the location of the facility with respect to the Saturn V space vehicle launch com-1

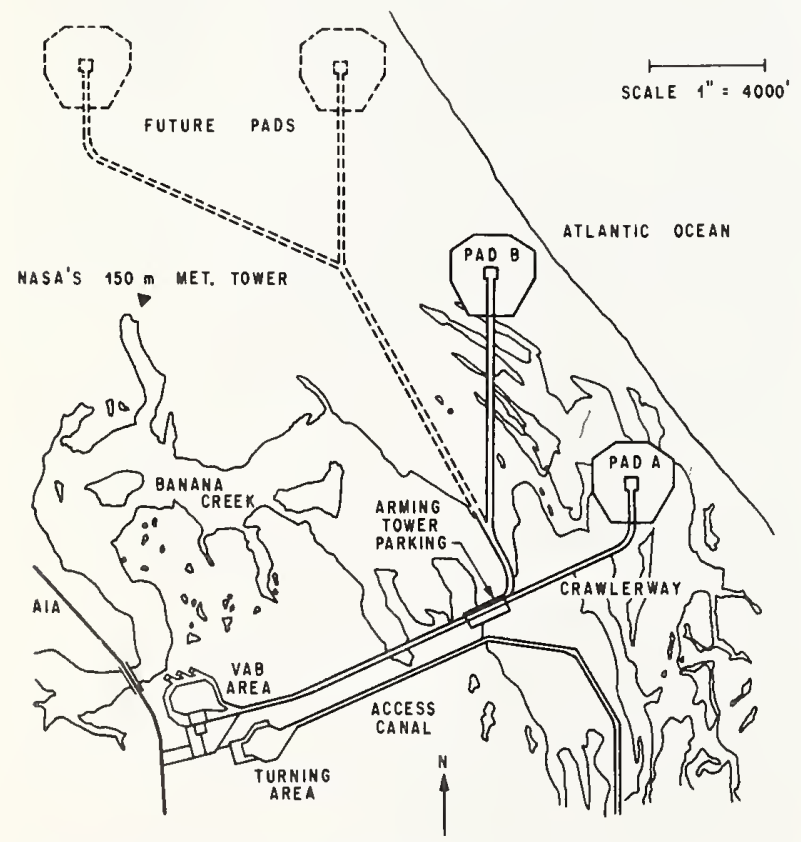

Figure 3. NASA Launch Complex 39, Kennedy Space Center, Florida. plex 39. Located about 3 miles from the Atlantic Ocean, the tower is situated in a well-exposed area free of near-by structures which could interfere with the air flow.

The aerial photograph (Fig. 4) of the terrain surrounding the tower (point $T$ ) was taken at $3,500 \mathrm{ft}$ above mean sea level. In the quadrant from approximately $300^{\circ}$ north azimuth with respect to the tower, clockwise around to $90^{\circ}$, the terrain is homogeneous and is covered with vegetation about $1 / 2$ to $1 \frac{1}{2}$ meters high. Another homogeneous fetch with the same type of vegetation occurs in the $135^{\circ}$ to $160^{\circ}$ quadrant. The areas $\mathrm{A}\left(230^{\circ}\right.$ to $\left.300^{\circ}\right), \mathrm{B}\left(90^{\circ}\right.$ to $\left.135^{\circ}\right)$, and $\mathrm{C}\left(160^{\circ}\right.$ to $\left.180^{\circ}\right)$ are covered with trees from about 10 to 15 meters tall. The fetch from the tower to areas $\mathrm{A}$ or $\mathrm{C}$ is about 200 meters, and the fetch to area $B$ is about 450 meters. The height of the vegetation over these fetches ranges from $1 / 2$ to $11 / 2$ meters, as in the area to the north of the tower. To the south-southwest in the $180^{\circ}$ to $230^{\circ}$ quadrant 225 meters from the tower, there is a body of water called Happy Creek.

\subsection{Instrumentation}

The complete tower facility comprises two towers, one 18 meters and the other 150 meters high (see Fig. 5). The levels on both towers are instrumented with Climet (Model Cl-14) wind sensors. Temperature sensors, Climet (Model-016) aspirated thermocouples, are located at the 3- and 18-meter levels on the small tower and at the 30-, 60-, 120-, and 150-meter levels on the large tower. Foxboro (Model F-2711AG) dewpoint temperature sensors are located at the 60 - and 150-meter levels on the large tower and at the 3-meter level on the 18-meter tower. Wind speed and direction data can be recorded on both paper strip charts and analog magnetic tapes with an Ampex FR-1200 fourteen-channel magnetic tape recorder which uses a 14 -in reel. The temperature and dewpoint data are recorded on paper strip charts. To avoid tower interference of the flow, the large tower is instrumented with two banks of wind sensors. The details of how and when one switches from one bank of instrumentation to the other bank is discussed by Kaufman and Keene in Reference 5 . During a test in which the wind data are stored on magnetic tape, only one bank of instrumentation is used. This avoids interruption of the wind data signals within any magnetic tape recording period, and thus avoids data-processing difficulties when converting analog tapes to digital tapes. 


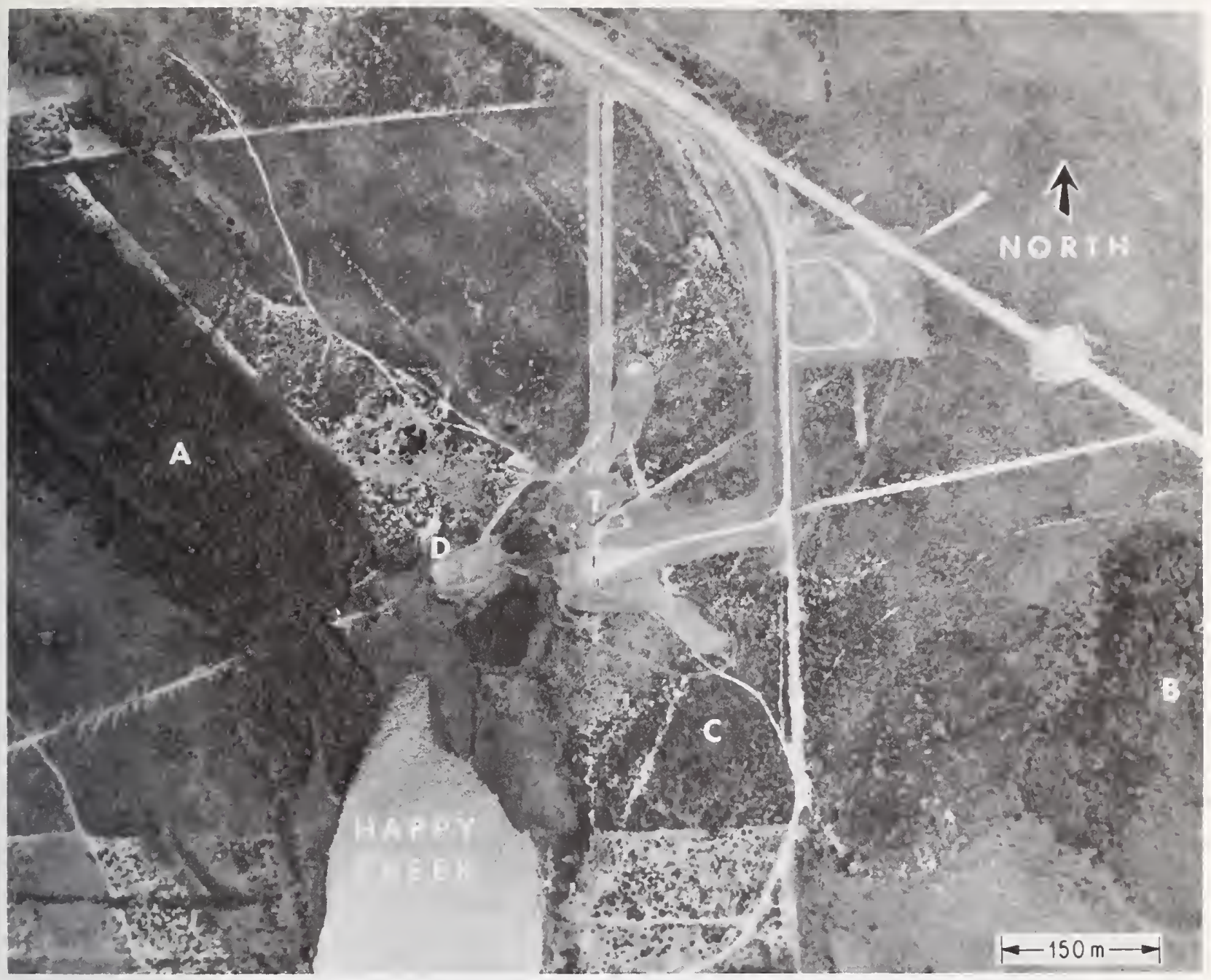

Figure 4. Aerial plan view of the terrain surrounding the NASA 150 -meter meteorological tower.

\subsection{Surface Roughness Length $\left(\mathrm{z}_{0}\right)$}

In an earlier report, Fichtl [6] discussed the surface roughness length configuration associated with the NASA meteorological tower. This analysis was based upon wind profile laws that are consistent with the Monin-Obukhov similarity hypothesis. The calculations of $z_{0}$ were based on wind data obtained at the 18- and 30-meter levels and on temperature data obtained at the 18 - and 60-meter levels. Most of the measurements were obtained during the hours of 0700 and $1600 \mathrm{EST}$, and the gradient Richardson numbers at 23 meters (geometric height between 18 and 30 meters) for the 39 cases ranged between -5.82 and +0.079 . The results of these calculations, shown in Figure 6, show the effect the terrain features (see Section IIIa) have upon the surface roughness.

\section{Design Wind Profiles}

To calculate wind loads on space vehicles, the engineer requires specific information about the wind profile. As pointed out in Section II, the fundamental wind statistics for the Kennedy Space Center are specified in terms of peak wind speeds for various periods of exposure (hour, day, month, etc.) at a reference height of 10 meters. A statistical peak wind profile model is used to extrapolate this information into the vertical, and the mean wind profile for various averaging periods is obtained by applying gust factors.

\subsection{Peak Wind Profiles}

To develop a peak wind profile model, about 6,000 hourly peak wind speed profiles measured during 1967 at the tower were analyzed. The data seemed 
BOOM HEIGHTS

(meters)

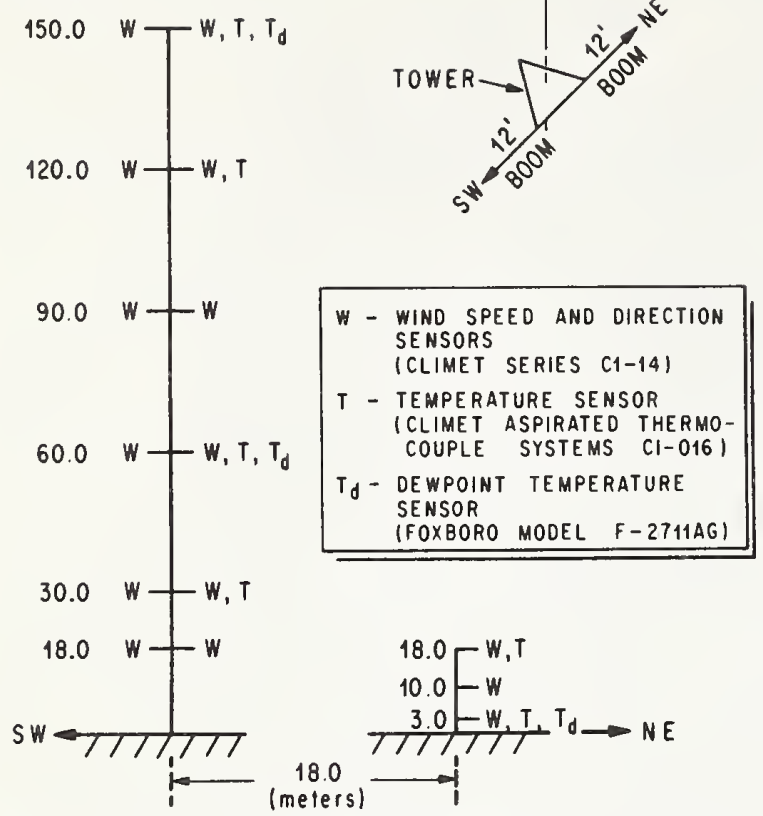

Figure 5. Sehematie diagram of the location of instrumentation of the NASA 150-meter meteorologieal tower at Kennedy Spaee Center, Florida.

to show that the variation of the peak wind speed in the vertical, below 150 meters, could be described with a power law relationship given by

$$
u(z)=u_{18}(z / 18)^{k},
$$

where $u(z)$ is the peak wind speed at height $z$ above natural grade and $u_{18}$ is a known peak wind speed at $z=18$ meters. The parameter $k$ was determined for each profile by a least-squares analysis of the data.

At low wind speeds on the order of $2 \mathrm{~m} \mathrm{sec}^{-1}$, the values varied from about -0.05 to 0.15 . Negative

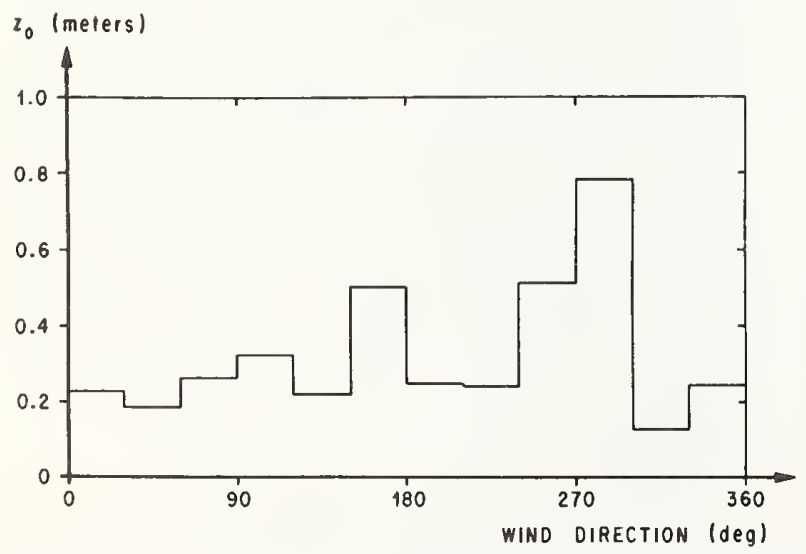

Figure 6. Distribution of the surface roughness length at the NASA 150-meter meteorologieal tower site. values of $k$ occurred for approximately $8 \%$ of the cases in the sample. One should keep in mind that we are analyzing peak wind profiles and that it is possible for the peak wind speed at some or all of the levels about 18 meters to be less than the 18-meter level peak wind speed resulting in negative values of $k$. This is not to imply that the associated mean wind speeds decrease in the vertical.

A statistical analysis of the data revealed that, for engineering purposes, $k$ is distributed normally for any particular value of the peak wind speed at the 18-meter level. Thus, for a given percentile level of occurrence, it was found that, for peak wind speeds at the 18-meter level less than approximately $2 \mathrm{~m} \mathrm{sec}^{-1}, k$ is equal to a constant, while for peak wind speeds greater than $2 \mathrm{~m} \mathrm{sec}^{-1}$,

$$
k=b u_{18}^{-3 / 4}
$$

where $b$ is a parameter that is distributed normally with mean value $\bar{k}$ and variance $\sigma$ equal to 0.52 and 0.36 and $u_{18}$ is in meters per second. The distribution of $k$ as a function of $u_{18}$ is shown in Figure 7 .

To apply Eqs. (1) and (2) to the peak wind statistics valid at 10 meters, Eq. (1) is evaluated at $z=10 \mathrm{~m}$, and it is assumed that the resulting relationship can be inverted to yield $u_{18}$ as a function of the 10-meter level peak wind speed $u_{10}$ for a

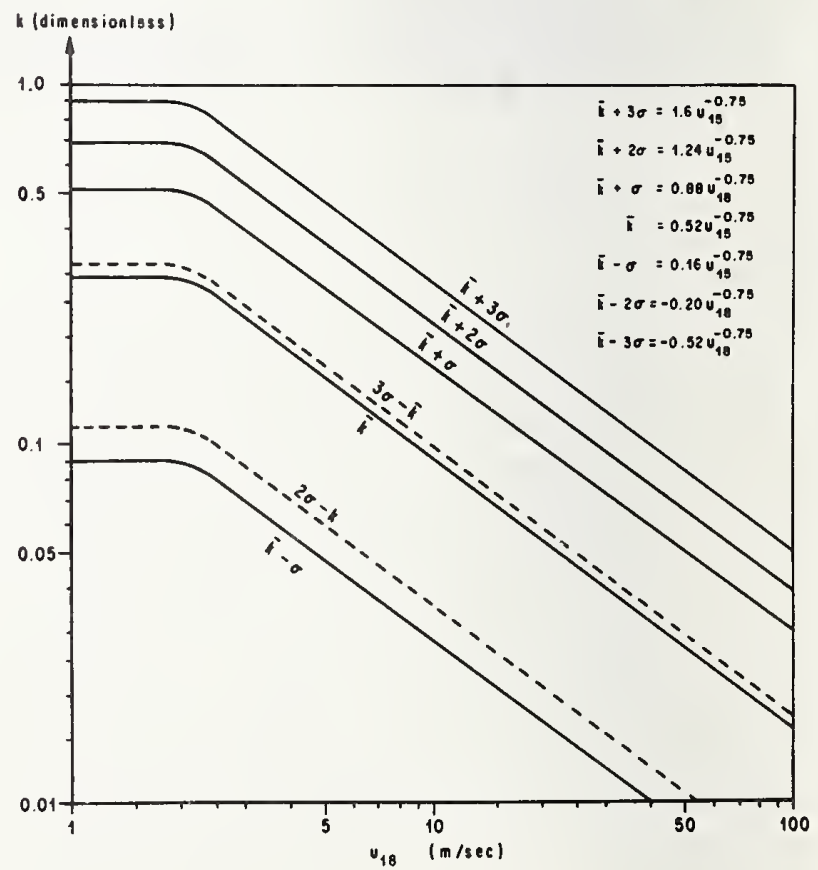

Figure 7. The mean, $\pm \sigma, \pm 2 \sigma$, and $\pm 3 \sigma$ values of $k$ as funetions of the peak wind speed $u_{18}$ at the 18-meter level. 
fixed value of $b$. This function is then combined with Eq. (2) to yield $k$ as a function of $u_{10}$ for a given value of $b$. The validity of this inversion process is open to question because Eq. (1) is a stochastic relationship. However, preliminary analyses of profiles that include peak wind information obtained at the 10-meter level seem to show that this inversion is valid.

The current design practice at MSFC is to use the $\bar{k}+3 \sigma$ value of $k$ to determine operational limits for space vehicles. Thus, if a space vehicle designed to withstand a particular value of the peak wind speed at the 10-meter level is exposed to that peak wind speed, the vehicle has at least a 99.87 percent chance of withstanding the associated peak wind speed profile.

\subsection{Alternative Approach}

At the present time, we are developing procedures to extrapolate peak wind speed distributions valid at the 10 -meter level to other levels. Consider a peak wind speed probability density function (p.d.f.) $p_{1}\left(u_{10}\right)$ valid at the 10 -meter level for a given period of exposure, say, one hour. The probability that $u_{10}$ is less than $u_{10 c}$ is given by

$$
p\left(u_{10}<u_{10 c}\right)=\int_{0}^{u_{10 c}} p_{1}\left(u_{10}\right) d u_{10} .
$$

To obtain the peak wind speed p.d.f., at level $z$, we express Eq. (1) in the form

$$
u_{10}\left(u_{z}, b\right)=u_{z}(10 / z)^{b\left[u_{18}\left(u_{z}, b\right)\right]-3 / 4},
$$

where we assume that Eqs. (1) and (2) can be inverted to yield $u_{18}$ as a function of $b$ and the peak wind at level $z$ denoted by $u_{z}$. Eq. (4) permits us to express Eq. (3) in the form

$p\left(u_{z}<u_{z c} \mid b\right)=\int_{0}^{u_{z c}} p_{1}\left(u_{10}\left(u_{z}, b\right)\right)\left(\frac{\partial u_{10}}{\partial u_{z}}\right)_{b} d u_{z}$

where $p\left(u_{z}<u_{z c} \mid b\right)$ is the conditional probability that $u_{z}$ is less than $u_{z c}$, given $b$ and $p\left(u_{z}<u_{z c} \mid b\right)=$ $p\left(u_{10}<u_{10 c}\right)$. Thus, the integrand in Eq. (5) is the conditional p.d.f. of $u_{z}$, given $b$, and is given by

$$
p_{2}\left(u_{z} \mid b\right)=p_{1}\left(u_{10}\left(u_{z}, b\right)\right)\left(\frac{\partial u_{10}}{\partial u_{z}}\right)_{b} .
$$

The quantity $\left(\partial u_{10} / \partial u_{z}\right)_{b}$, a function of $u_{z}$ and $b$, can be calculated by differentiating Eq. (4). The joint p.d.f. of $u_{z}$ and $b$ is given by

$$
p_{2}\left(u_{z}, b\right)=p_{1}\left(u_{10}\left(u_{z}, b\right)\right)\left(\frac{\partial u_{10}}{\partial u_{z}}\right)_{b} p(b),
$$

where $p(b)$ is the p.d.f. of $b$, a known function. Upon integrating Eq. (7) over the range of $b$, we obtain the marginal p.d.f. of $u_{z}$ :

$$
p_{2}\left(u_{z}\right)=\int_{-\infty}^{\infty} p_{1}\left(u_{10}\left(u_{z}, b\right)\right)\left(\frac{\partial u_{10}}{\partial u_{z}}\right) p(b) d b .
$$

At the present time, we are calculating these intcgrals numerically for the annual hourly peak wind speed data. To calculatc the monthly and seasonal peak wind speed statistics, we require the associated monthly and seasonal distributions of $k$. Programs are now being written to calculate thesc statistics.

Once we have determined the p.d.f. of the peak wind speed as a function of height, we can then calculate design peak wind-speed profile envelopes for various percentile levels of occurrence.

\subsection{Instantaneous Extreme Wind Profiles}

Because the probability that the hourly peak wind speeds at all levels occur simultaneously is small, the practice of using peak wind profiles introduces some conservatism into the design criteria. This section estimates the amount of conservatism involved.

To gain some insight into this question, about 35 hours of digitized magnetic tapc data were analyzed. The data were digitized at 0.1 -second intervals in real time and partitioned into $0.5-, 2-, 5-$, and $10-$ minute samples. The vertical average peak wind speed $\bar{u}_{P}$ and the 18 -meter mean wind $\bar{u}_{18}$ were calculated for each sample. In addition, the instantaneous vertical average wind speed time history at 0.1-second intervals was calculated for each sample, and the peak instantaneous vertical average wind speed $\bar{u}_{I}$ was selected from each sample. The quantity $\bar{u}_{T} / \bar{u}_{P}$ was then interpreted to be a measure of how well the peak wind profile statistics at the 10 -meter level approximate the instantaneous extreme wind profile statistics.

In Figure 8 , a plot of $\bar{u}_{I} / \bar{u}_{P}$ as a function of $\bar{u}_{18}$, the data points tend to scatter about a mean value

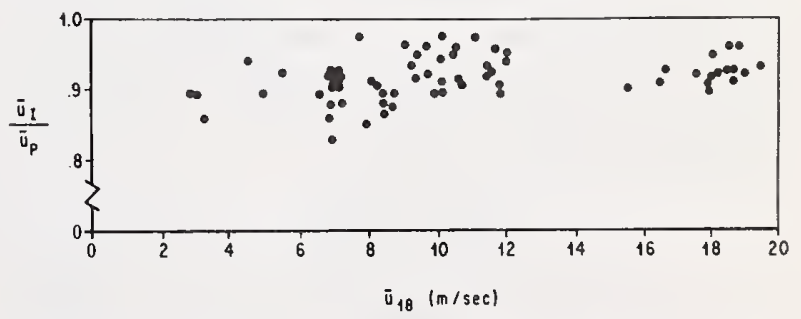

Figure 8. The ratio $\bar{u}_{I} / \bar{u}_{P}$ as a function of the mean wind speed $\bar{u}_{18}$ at the 18-meter level for an averaging time equal to 10 minutes. 


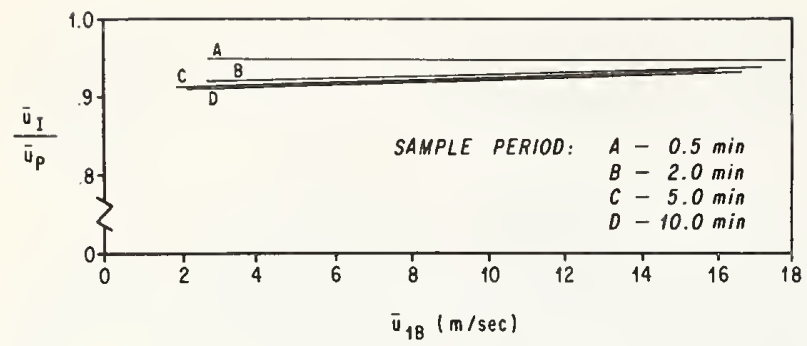

Figure 9 The ratio $\bar{u}_{I} / \bar{u}_{P}$ as a function of the mean wind speed $\bar{u}_{18}$ at the 18 -meter level for averaging times equal to $0.5(A), 2(B), 5(C)$, and 10 minutes $(D)$.

of $\bar{u}_{I} / \bar{u}_{P} \simeq 0.93$. Since drag loads are proportional to the square of the velocity, this mean value implies that the peak wind profile may be overestimating the loads by about $14 \%$. However, the fact that some of the data points have values equal to $0.9 \mathrm{~S}$ could mean an overestimate of the loads by only $4 \%$. Figure 9 gives the average values of $\bar{u}_{I} / \bar{u}_{P}$ as a function of $\bar{u}_{18}$ for different averaging times $(0.5,2$, 5 , and 10 minutes).

\subsection{Gust Factor}

The gust factor $G$ is defined as

$$
G=u / \bar{u},
$$

where $u$ is the peak wind speed within a data record of length $t$ in time and $\bar{u}$ is the mean wind speed associated with the data record. A simple theory can be constructed to aid in understanding the behavior of the gust factor. If $\sigma$ denotes the variance of the fluctuations of velocity about the mean wind specd, then $\bar{u}+3 \sigma$ is an estimate of the peak wind speed; therefore,

$$
G=1+\frac{3 \sigma}{\vec{u}} .
$$

The variance is related to the friction velocity, $u_{*}$, through the relationship

$$
\sigma=A(R i, t) u_{*},
$$

where $A$ is a function of the Richardson number, $R i$, and the averaging time, $t$. The Richardson number is given by

$$
R i=\frac{\frac{g}{\bar{T}}\left(\frac{g}{C_{p}}+\frac{d \bar{T}}{d z}\right)}{\left(\frac{d \bar{u}}{d z}\right)^{2}},
$$

where $\bar{T}$ and $\vec{u}$ denote the mean Kelvin temperature and wind speed at height $z, g$ is the acceleration of gravity, and $C_{p}$ is the specific heat of dry air at constant pressure $\left(g / C_{p} \simeq 9 . S^{\circ} \mathrm{K} / \mathrm{km}\right)$. In approximately the first 30 meters of the boundary layer, the wind profile is given by

$$
\bar{u}=\frac{u_{*}}{k_{1}}\left(\ln \frac{z}{z_{0}}-\psi(R i)\right)
$$

where $k_{1}$ is von Karman's constant with numerical value approximately equal to $0.4, z_{0}$ is the surface roughness length and $\psi(R i)$ is a universal function of $R i$.

Combining Eqs. (9), (10), (11) and (13), we find that

$$
G=1+\frac{3 k_{1} A(R i, t)}{\ln \frac{z}{z_{0}}-\psi(R i)}
$$

For a neutral atmosphere, $R i=0$, and $\psi$ vanishes, so that

$$
G=1+\frac{3 k_{1} A(0, t)}{\ln \frac{z}{z_{0}}}
$$

We may conclude from this relationship that the gust factor decreases as the height increases. This result is also qualitatively true for unstable air $(R i<0)$. As the averaging time decreases, the variance will decrease so that $A$ is a decreasing function of the averaging time and thus, we may conclude from (14) that $G$ is an increasing function of the averaging time.

The functions $\psi$ and $A$ are monotonically decreasing functions of the Richardson number; $\psi$ vanishes in neutral $(R i=0)$ air, while $A$ is positive definite. Thus, as the Richardson number decreases, or rather, as the air becomes more unstable, the gust factor increases.

Let us now consider a typical daytime situation at Cape Kennedy. At low wind speeds the air is unstable and $G$ is large. However, as the wind speed increases, the wind shear $(d \bar{u} / d z)$ increases, causing the Richardson number to tend to zero from the unstable side of $R i=0$. Thus, an increase in the wind speed will tend to lower the gust factor in view of the dependence of the gust factor upon stability. In a typical nighttime situation, the stratification is stable, and the Richardson number is usually positive. As the wind speed increases, the 
Richardson number tends to zero from the stable side of $R i=0$. This means that the gust factor will increase as the wind speed increases. In both eases the limiting value of the gust factor will be that of a neutral atmosphere $(R i=0)$ as given by Eq. (15).

In view of these considerations, a gust factor model for the Kennedy Space Center was developed with 181 hours of afternoon turbulence data encompassing a broad range of wind speed conditions. Gust factors were calculated for averaging times $(t)$ equal to $0.5,1,2,5,10$ and 60 minutes. It was assumed that the gust factor is a function of the averaging time $t$, and the peak wind speed $u_{18}$ at the 18-meter level. The peak wind speed at the 18meter level plays the role of a stability parameter. It was found that the expected value of the gust factor at any level between 18 and 150 meters can be represented as

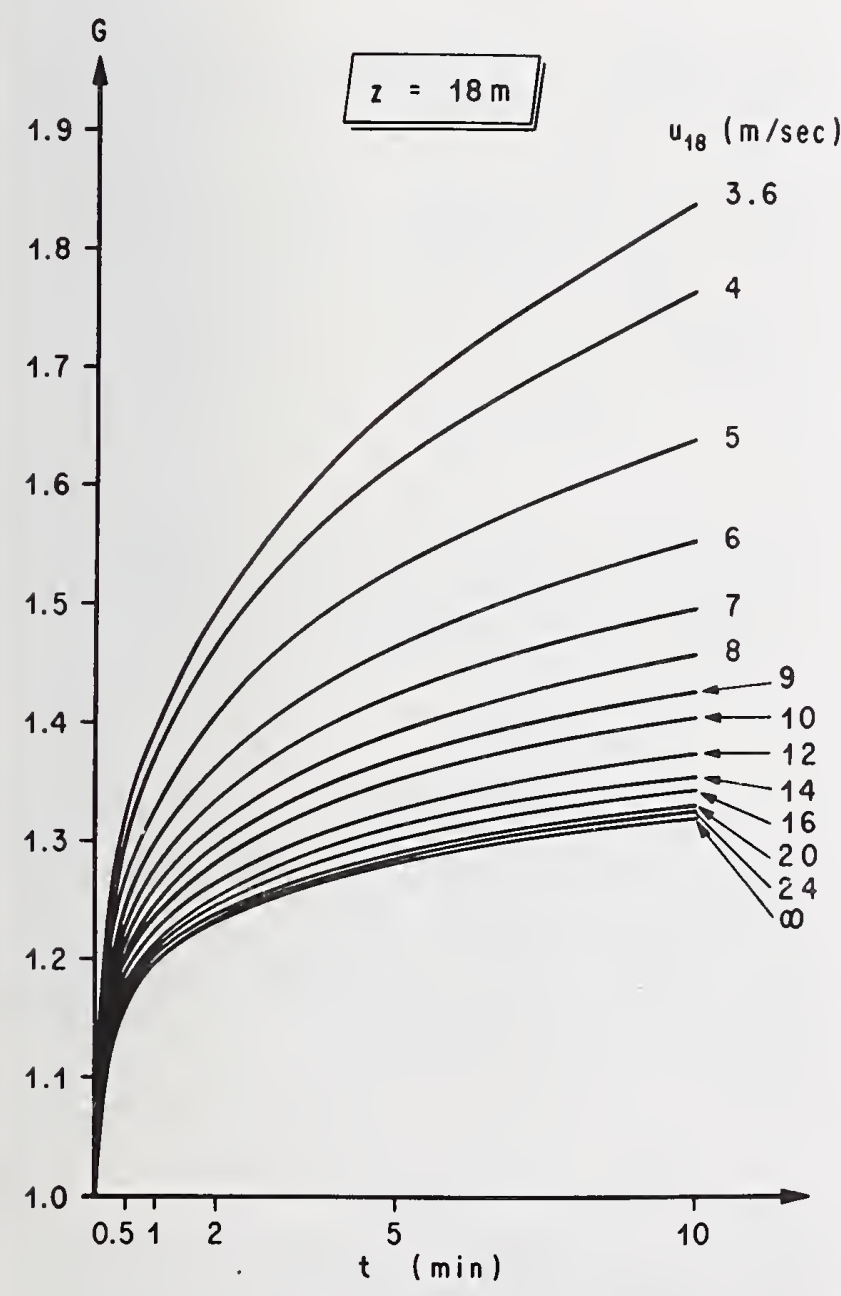

Figure 10. The gust factor $G$ at the 18-meter level as a function of the averaging time for various peak wind speeds at the 18-meter level.

$$
G=1+\frac{1}{g_{0}}(18 / z)^{n},
$$

where $z$ is the height in meters. In this equation the parameters $p$ and $g_{0}$ are given by

$$
p=0.283-0.435 e^{-0.2 u_{18}}
$$

and

$g_{0}=1.98+0.085\left(\ln \frac{t}{10}\right)^{2}-0.329 \ln \frac{t}{10}-1.887 e^{-0.2 u 18}$,

where $t$ and $u_{18}$ have the units of minutes and meters per second. The dependence of the 18-meter level gust factor on the averaging time and the peak wind speed is shown in Figure 10, and the dependence of the 10-minute gust factor on the peak wind speed and height is given in Figure 11.

Within the range of variation of the data, the 1hour gust factor and the 10-minute gust factor were approximately equal, because the spectrum of the horizontal wind speed near the ground is characterized by a broad energy gap centered at a frequency approximately equal to 1 cycle $\mathrm{hr}^{-1}$ and typically extends over the frequency domain 0.5 cycles

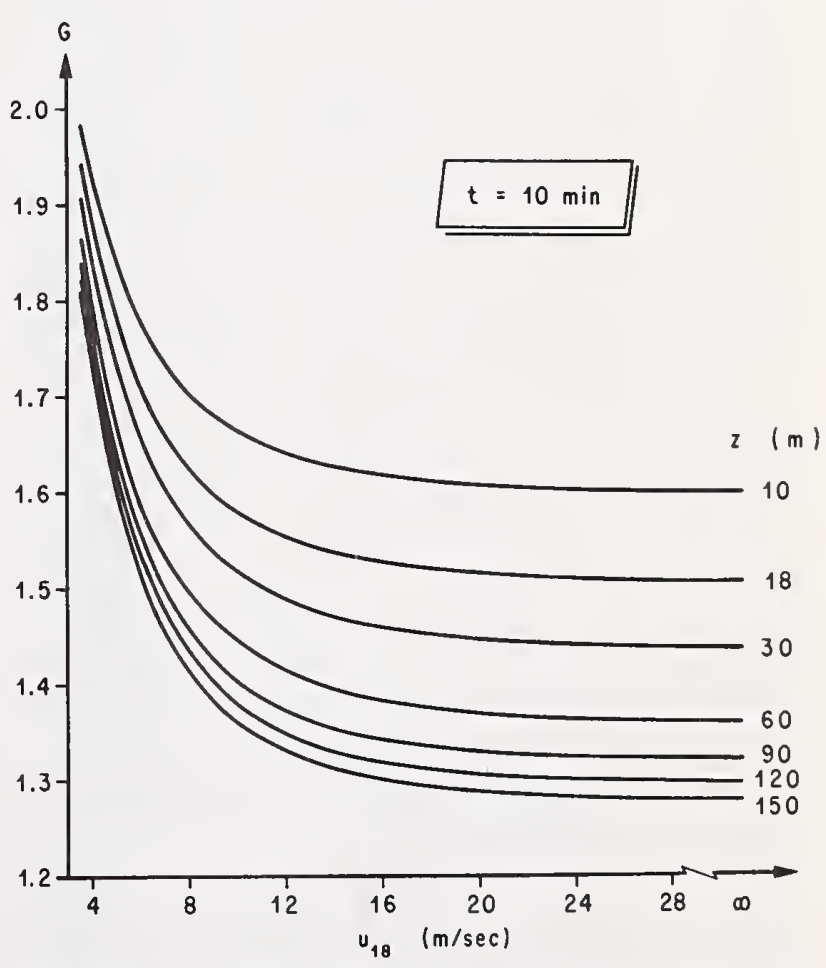

Figure 11. The gust factor $G$ as a function of the peak wind $u_{18}$ at the 18 -meter level for various heights above natural grade associated with a 10 -minute grand average. 


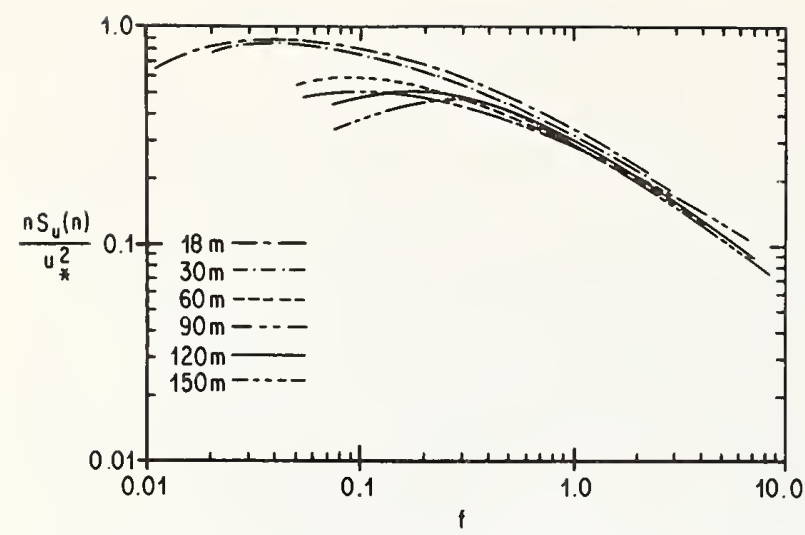

Figure 12. Dimensionless logarithmic longitudinal spectra for neutral wind conditions plotted in Monin coordinates.

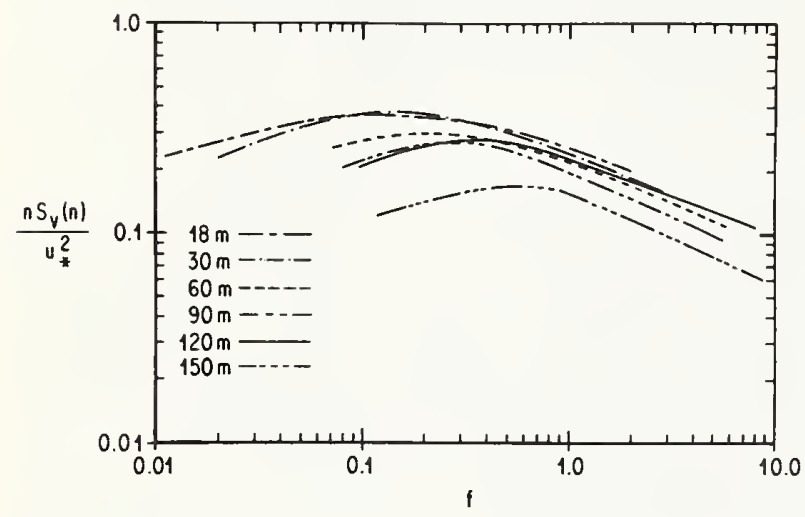

Figure 13. Dimensionless logarithmic lateral spectra for neutral wind conditions plotted in Monin coordinates.

the longitudinal and lateral components of turbulence are decreasing functions of $z$, so that by scaling the spectra with the surface value of the friction velocity, the scaled spectra at the upper levels fall below the 18-meter spectra.

By scaling the wave number with $z$, we have assumed that the integral scales of the longitudinal and lateral components of turbulence are proportional to $z$. This might be true in the Monin layer; however, in the Ekman layer one might suspect from the behavior of eddy coefficients [14] that, if the local integral scales have a vertical variation, then they should increase at a rate slower than $z$. In addition, we have no knowledge that the integral scales of the longitudinal and lateral spectra should have the same vertical variation. However, the data appear to show that Monin coordinates will collapse spectra with various turbulence intensities at any particular level in the vertical.

To produce a vertical collapse of the data, it was assumed, for engineering purposes, that the spectra in Monin coordinates are shape-invariant in the vertical. This hypothesis appears to be reasonable and permits a practical approach to developing an engineering spectral model of turbulence.

\subsection{The Longitudinal Spectrum}

The vertical variation of the dimensionless wave number $f_{m u}$ associated with the peak of the logarithmic spectrum $S_{u}$ scaled in Monin coordinates is given in Figure 14. A least-squares analysis of the data in this figure yields the result

$$
f_{m u}=0.03(z / 18),
$$

where $z$ is in meters. A plot of $n S_{u}(n) / u_{*}{ }^{2}$ versus $f / f_{m u}$ will shift the spectra at the various levels, so that all the peaks of the logarithmic longitudinal spectra are located at $f / f_{m u}=1$. Values of $f_{m u}$ from other tower sites are indicated in Figure 14.

The average ratio $\beta_{u}$ of the shifted spectrum at level $z$ and the 18-meter spectrum,

$$
\left(S_{u}\left(f / f_{m u}, z\right) / S_{u}\left(f / f_{m u}, 18\right)\right),
$$

is shown in Figure 15. A least-squares analysis of these data yielded the result

$$
\beta_{u}=(z / 18)^{-0.63},
$$

where $z$ is in meters. A plot of $n S_{u}(n) / \beta_{u} u_{*}{ }^{2}$ versus $f / f_{m u}$ will collapse the longitudinal spectra. The collapsed longitudinal data are plotted as a function

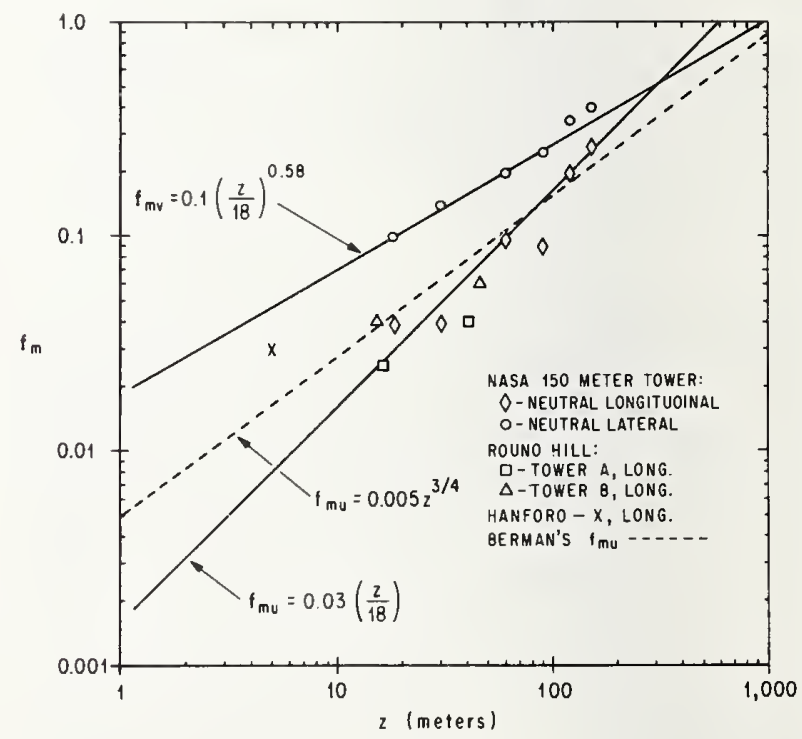

Figure 14. Vertical distributions of the dimensionless frcquencies $f_{m u}$ and $f_{m v}$ associated with the peak of the logarithmic longitudinal and lateral spectra for neutral stability conditions. 


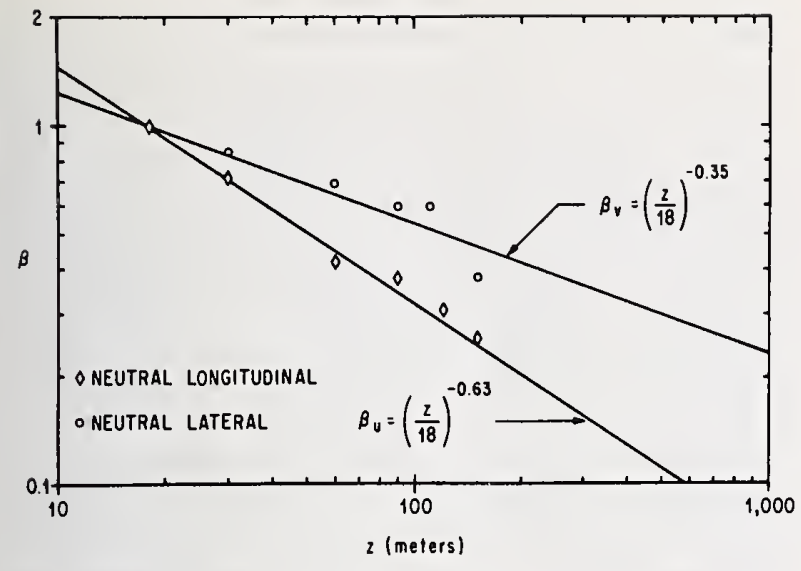

Figure 15. The vertical distribution of the collapsing factors $\beta_{u}$ and $\beta_{v}$ for neutral stability conditions.

of $0.03 f / f_{m u}$ in Figure 16 .

The function

$$
\frac{n S_{u}(n)}{\beta_{u} u_{*}^{2}}=\frac{C_{u} f / f_{m u}}{\left(1+1.5\left(f / f_{m u}\right)^{r_{u}}\right)^{5 / 3 r_{u}}}
$$

was selected to represent the longitudinal spectrum, where $C_{u}$ and $r_{u}$ are positive constants, determined by a least-squares analysis. For sufficiently small values of $f, n S_{u}(n) / \beta_{u} u *^{2}$ asymptotically behaves like $f / f_{m u}$ which is the correct behavior for a onedimensional spectrum. At large values of $f$, $n S_{u}(n) / \beta_{u} u_{*}{ }^{2}$ asymptotically behaves like

$$
\left(f / f_{m u}\right)^{-2 / 3} \text {, }
$$

consistent with the concept of the inertial subrange. The maximum value of (32) occurs at $f=f_{m u}$. Various authors have suggested formulae like (32) to represent the longitudinal spectrum. However, most of the representations have only one adjustable parameter available, while Eq. (32) has two: $C_{u}$ and $r_{u}$. In this light (32) appears to be superior. Upon setting $r_{u}=5 / 3$, we obtain the form of the longitudinal spectrum suggested by Panofsky [7] to represent the strong wind spectra of Davenport [15]. Von Karman's longitudinal spectrum [16] can be obtained by setting $r_{u}=2$. A least squares analysis of the longitudinal data in Figure 16 revealed that $C_{u}=8.641$ and $r_{u}=0.845$.

\subsection{The Lateral Spectrum}

The lateral spectra $S_{v}$ can be collapsed with a procedure like the one used for the longitudinal spectra. However, to determine an analytical expression for the lateral spectrum, special attention must be paid to the inertial subrange to guarantee that $S_{u} / S_{v}=3 / 4$ [17]. This requirement can be derived from the mass continuity equation for incompressible flow subject to the condition that the eddies are isotropic in the inertial subrange. The experimental values of $f_{m v}$ and $\beta_{v}$ are given in Figures 14 and 15. These data show that $f_{m v}$ and $\beta_{v}$ can be represented as power laws as for the longitudinal spectra. The function

$$
\frac{n S_{v}(n)}{\beta_{v} u_{*}^{2}}=\frac{C_{v} f / f_{m v}}{\left(1+1.5\left(f / f_{m v}\right)^{r_{v}}\right)^{5 / 3 r_{v}}}
$$

was used to represent the scaled spectra, where $C_{0}$ and $r_{v}$ are positive constants. This function behaves like the one chosen for the longitudinal spectrum.

For sufficiently large values of $f$, the asymptotic behavior of the ratio between Eqs. (32) and (33) is given by

$$
\frac{S_{u}}{S_{v}} \sim \frac{C_{u}}{C_{v}} \frac{\beta_{u}}{\beta_{v}}\left(\frac{f_{m u}}{f_{m v}}\right)^{2 / 3}(3 / 2)^{5 / 3\left(1 / r_{v}-1 / r_{u}\right)} .
$$

In the inertial subrange we must have $S_{u} / S_{v}=3 / 4$, so that upon substituting this ratio into (34) we obtain a relationship that can be used as a constraint in the determination of values of $C_{v}$ and $r_{v}$ and functions to represent $\beta_{v}$ and $f_{m v}$. The values $C_{v}=8.686$ and $r_{v}=0.512$, and the functions

$$
f_{m v}=0.1(z / 18)^{0.58}
$$

and

$$
\beta_{v}=(z / 18)^{-0.35}
$$

along with the longitudinal parameters will satisfy condition (34) and simultaneously give a good fit to the data ( $z$ is in meters). The collapsed lateral spectra and the functions given by (32) and (33) are shown in Figure 16.

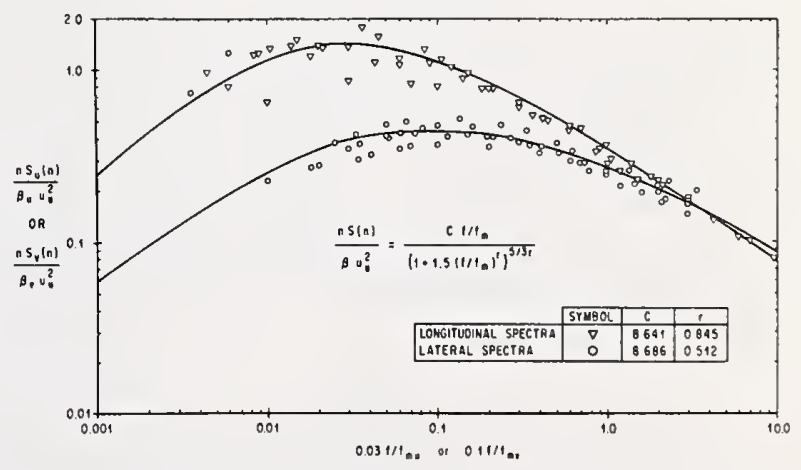

Figure 16. Dimensionless logarithmic longitudinal and lateral spectra as functions of $0.03 f / f_{m u}$ and $0.1 f / f_{m v}$ for neutral stability conditions. 


\subsection{Engineering Application}

To apply the spectrum of turbulence to engineering problems, we first select the design peak wind speed for a prescribed level of risk. The peak wind speed profile is established by selecting the 99.57 percentile (mean $+3 \sigma$ ) value of $k$, and the mean wind profile is obtained by "backing off" from the peak wind profile with the appropriate gust factor profile. The friction velocity $u_{*}$ is calculated with Eq. (21) for neutral wind conditions ( $R i=0$, $\psi(0)=0)$. Once the mean wind speed profile and the friction velocity are known, the longitudinal and lateral spectra are completely specified. This is the procedure presently used at MSFC.

Thus far, we have been talking about the spectra of the longitudinal and lateral components of turbulence. Actually, it is the spectra of the square of these components that are important from a loads viewpoint. If we assume that these components individually constitute Gaussian processes, then it is possible to express the power spectra of $\left(\bar{u}+u^{\prime}\right)^{2}$ or $v^{\prime 2}$ with the Wiener-Khintchine theorem in the form

$$
\begin{aligned}
S_{2}(n)=\left[\left(\bar{u}^{4}+2 \bar{u}^{2} \sigma^{2}\right) \epsilon+\sigma^{4}\right] \delta(n)+4 \epsilon \bar{u}^{2} S_{1}(n) & \\
& +2 \int_{-\infty}^{\infty} S_{1}(n-\xi) S_{1}(\xi) d \xi,
\end{aligned}
$$

where $S_{2}(n)$ is defined in the interval $-\infty<n<\infty$, $\epsilon=1$ (longitudinal loads) or 0 (lateral loads) and $\delta(n)$ is the Dirac delta function. In this equation, the variance of the turbulence is given by

$$
\sigma^{2}=\int_{0}^{\infty} S(n) d n,
$$

where $S(n)$ is the longitudinal or lateral spectrum as defined by Eqs. (32) and (33) and

$$
S_{1}(n)=\frac{S(|n|)}{2}
$$

The details of the derivation of Eq. (37) can be found in a paper by Wood and Berry [18].

To produce the convolution integral in Eq. (37) by numerical procedures is straightforward. The right-hand side of Eq. (37) is directly proportional to the spectrum of the longitudinal or lateral drag forces, which are the required inputs for vehicle response studies.

\section{Conclusions}

Development of space vehicle wind loads design criteria is not simple, but is a rather complicated procedure requiring the designer and the atmospheric scientist to work as a team. The engineer must specify the risks he is willing to accept, while the atmospheric scientist must produce a wind model from which it is possible to determine the appropriate forcing functions for that accepted risk. The model presented here for Cape Kennedy, Florida, serves this purpose in that risk values can be applied to the occurrence of peak wind speeds at a reference level for a given period of exposure; and if these design peak wind speeds occur, an upper bound risk value of structural failure can be estimated from statistical information about the wind profile shape $(k)$. By applying gust factors for an appropriate averaging period, a peak wind speed environment can be partitioned into mean wind and turbulence environments.

\section{References}

[1] Smith, O. E., L. W. Falls, and S. C. Brown, "A Statistical Analysis of Winds for Aerospace Vehicle Design, Mission Planning, and Operations," Research Achievements Review, Vol. II, Report No. 10, Terrestrial and Space Environment at MSFC, NASA TM X-53706, George C. Marshall Spacc Flight Center, Huntsville, Alabama, 1967.

[2] Fisher, R. A., Contributions to Mathematical Statistics, John Wiley and Sons, Inc., New York, 1950.

[3] Gumbel, E. J., Statistics of Extremes, Columbia University Press, New York, 1958,

[4] Thom, H. C. S., "Distribution of Extreme Winds in the United States," Journal of the Structural Division, Proceedings of the American Society of Civil Engineers, April 1960.

[5] Kaufman, J. W. and L. F. Keene, "NASA's 150-Meter Meteorological Tower Located at Cape Kennedy Florida " NASA TM X-53259, George C. Marshall Space Flight Center, Huntsville, Alabama, May 12, 1965.

[6] Fichtl, G. H., "An Analysis of the Roughness Length Associated with the NASA 150-Meter Metcorological Tower," NASA TM X-53690, George C. Marshall Space Flight Center, Huntsville, Alabama, January 3, 1968.

[7] Lumley, J. L. and H. A. Panofsky, The Structure of Atmospheric Turbulence, John Wiley and Sons, 1964.

[8] Davenport, A. G., "The Relationship of Wind Structure to Wind Loading," presented at the Meeting on Ground Wind Load Problems in Relation to Launch Vehicles, NASA Langley Research Center, June 7-8, 1966.

[9] Blackman, R. B. and J. W. Tukey, The Measurement of Power Spectra, Dover, New York, 1958.

[10] Pasquill, F., Atmospheric Diffusion, D. van Nostrand Company, Ltd., New York, 1962.

[11] Monin, A. S., "On the Similarity of Turbulence in the Presence of a Mean Vertical Temperature Gradient," J. Geophys. Res., Vol. 64, 1959, pp. 2196-2197.

[12] Berman, S., "Estimating the Longitudinal Wind Spectrum Near the Ground," Quart. J. Roy. Meteor. Soc., 91, 1965, pp. 302-317.

[13] Busch, N. E. and H. A. Panofsky, "Recent Spectra of Atmospheric Turbulence," Quart. J. Roy. Meteor. Soc., 94, 1968, pp. 132-148. 
[14] Blackadar, A. K. et al., "Flux of Heat and Momentum in the Planetary Boundary Layer of the Atmosphere," The Pennsylvania State University Mineral Industries Experiment Station, Dept. of Meteorology, Rept. under AFCRL Contract No. AF9604-6641, 1965.

[15] Davenport, A. G., "The Spectrum of Horizontal Gustiness Near the Ground in High Winds," Quart. J. Roy. Meteor. Soc., Vol. 87, 1961, pp. 194-211.

[16] Pritchard, F. E., C. G. Casterbrook, and G. E. McVehil, "Spectral and Exceedance Probability Models of Atmos- pheric Turbulence for Use in Areraft Design," TR AFFDL-TR-65-122, Air Force Flight Dynamics I aboratory, Rescarch and Technology Division, Air Force Systems Command, Wright-Patterson A PB, Ohio, 1965.

[17] Batchelor, C. K., The Theory of IIomogencous Turbulence, Cambridge University Press, New York, 1953.

[18] Wood, J. D. and J. G. Berry, "Random Excitation of Missiles Duc to Winds," Proc. of National Symposium on Winds for Acrospace Vehicle Design, Vol. 1, USAF Geophysics Res. Dir. A.F. Surveys in Geophysics, No. 140, Vol. 1, AFCRL-62-273(I), March 1962, pp. 125-138. 
SESSION 3.

\title{
EXPERIMENTAL AND THEORETICAL DETERMINATION OF AERODYNAMIC FORCES
}

\author{
Chairman: Professor A. G. Davenport \\ Faculty of Engineering Science \\ University of Western Ontario \\ London, Ontario, Canada
}





\title{
FLUCTUATING MOMENTS ON TALL BUILDINGS PRODUCED BY WIND LOADING
}

\author{
J. E. Cermak, ${ }^{*}$ W. Z. Sadeh, ${ }^{* *}$ and G. Hsi*** \\ Fluid Dynamics and Diffusion Laboratory \\ College of Engineering \\ Colorado State University \\ Fort Collins, Colorado 80521
}

\begin{abstract}
Wind loading on a 1:384 scale model of a building $666 \mathrm{ft}$ high was studied experimentally in a thick-boundary-layer wind tunnel. Measurements of mean velocity and turbulence intensity upstream of the model building verified that the wind tunnel flow was an adequate simulation of atmospheric-surface-layer flow over an urban area.

Mean pressure distributions and local pressure fluctuations were measured for a variety of upstream roughness conditions and wind directions. Use of a high frequency response pressuremeasuring system permitted rms and peak values of the local pressure fluctuations to be determined at numerous points on the building surface.

Emphasis was placed on direct measurement of mean and fluctuating overturning moments by means of a strain-gage dynamometer. A stiff model, $U / D n<1$, was used to obtain moments due to wind action alone. Peak values of the moment fluctuations were found to have a magnitude of $\pm 34 \%$ of the mean moment. Root-mean-square values of the moment fluctuations were also determined in an effort to relate the moment fluctuations to the measured pressure fluctuations.
\end{abstract}

Key words: Boundary layer; buildings; pressure fluctuations; instrumentation; overturning moments; wind loads; wind tunnel modeling.

\section{Introduction}

The study of aerodynamic forces on tall buildings produced by strong turbulent winds can now be accomplished through the use of scale models placed in a wind tunnel capable of simulating the atmospheric surface layer. Existing wind tunnels capable of simulating the mean velocity variation and turbulence structures with height up to about $1,600 \mathrm{ft}$ because of their special long test sections are located at Colorado State University [1] $]^{1}$ and the University of Western Ontario [2]. Past studies have been devoted primarily to determination of pressure coefficients for the mean wind, local pressure fluctuations, and in the case of an aeroelastic model, deflections of the buildings, e.g., Marshall and Cermak [3], Davenport and Isyumov [2] and Ostrowski, Marshall and Cermak [4].

Knowledge of the distribution of mean pressure coefficients permits calculation of mean overturning moments and mean shear while rms and peak values

* Professor-in-Charge, Fluid Mechanics Program.

** Assistant Professor of Engineering.

*** Research Associate.

${ }^{1}$ Figures in brackets indicate literature references at end of this paper. of local pressure fluctuations enable a proper choice of window glass and outer skin panelling to be made. However, these data do not give adequate information on a quantity of prime importance to the structural engineer, the fluctuating overturning moment. Mean pressure coefficients yield the mean overturning moment but the local pressure fluctuation data do not yield the fluctuations in overturning moment because the space correlation of pressure fluctuations on the building surface is not known.

The research reported in this paper emphasizes an exploratory effort to measure directly the fluctuating overturning moment produced by a turbulent wind. By using a high-frequency-response system the total moment, i.e., mean and fluctuating moment, was measured. The natural frequency of the strain-gage dynamometer-building shell system was about $200 \mathrm{~Hz}$. Furthermore, the structure was stiff enough, i.e., the reduced velocity $U / D n<1$, such that the measured moments are determined completely by the wind characteristics and not by structural dynamic action.

\section{Experimental Apparatus}

The main objective of this study was the direct measurement of the total overturning moment, i.e., 
mean and fluctuating moment. This was accomplished by using a suitable dynamic balance. The latter was designed to respond only to this moment.

Prior to moment measurement an investigation to obtain the local and overall wind loading on a rigid tall rectangular building was conducted. The latter is defined by both the mean and fluctuating values of pressure acting on the structure. Furthermore, for certain structural considerations the instantaneous peak value of pressure is also of interest. Therefore, an exploration of pressure distribution, i.e., mean, fluctuating (rms) and peak pressure, over a scaled building model was carried out in a wind tunnel.

The experimental objective could not be attained by simply placing an appropriate model in a regular aerodynamic wind tunnel $[5,6]$. A rather detailed discussion of this problem is reported in References 1, 2 and 7. It was desired to obtain a mean velocity profile similar to the natural wind. Moreover, a suitable thick turbulent boundary layer was necessary for simulating the real flow conditions. Consequently, the experiments were performed in an appropriate wind tunnel which is described later.

The flow around structures can be considered similar to flow around bluff bodies at relatively large Reynolds numbers. When the flow is simulated in a wind tunnel, dynamic similarity needs to be satisfied. For relatively large Reynolds numbers and sharp edged structures, i.e., Reynolds number on the order of $10^{5}$, the drag coefficient and, thus, the pressure distribution and the resulting forces are independent of the Reynolds number $[1,8]$. Since the Reynolds number of the experimental flow was of this order it follows that the flow pattern and, hence, the pressure distribution and the overturning moment are Reynolds-number independent. The former was based on the largest dimension of the building model cross section and/or on the equivalent (hydraulic) diameter of the model.

For these reasons, it was decided to use a simple model placed in a relatively thick turbulent boundary layer formed over a surface with roughness (upstream of buildings) scaled to the same scale as the structure of interest. A schematic diagram of the experimental arrangement and of the low-speed wind tunnel used is displayed in Figure 1 which also shows all important dimensions.

\subsection{The Model Building}

A scaled model of the two towers planned for the Atlantic-Richfield Plaza in Los Angeles was made of "Lucite" 0.375 in thick. The buildings are of

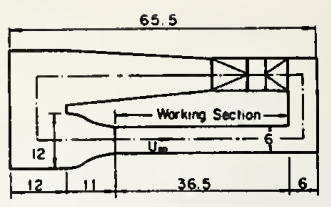

Tap View of the Low Speed Wind Tumel

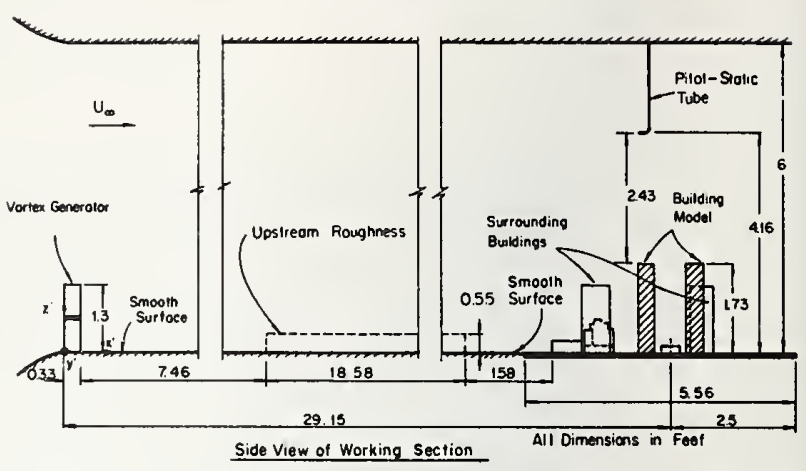

FigURE 1. Sketch of the experimental arrangement and the low-speed wind tunnel.

rectangular shape and $666 \mathrm{ft}$ high. A geometrical scale of 1:384 was employed. This scale was chosen for obtaining a meaningful simulation of the natural wind over a built-up urban area. The atmospheric boundary layer is about $1600 \mathrm{ft}$ thick, thus, about 2.4 times the building height. Therefore, a boundary layer about 50 in. in thickness was desired.
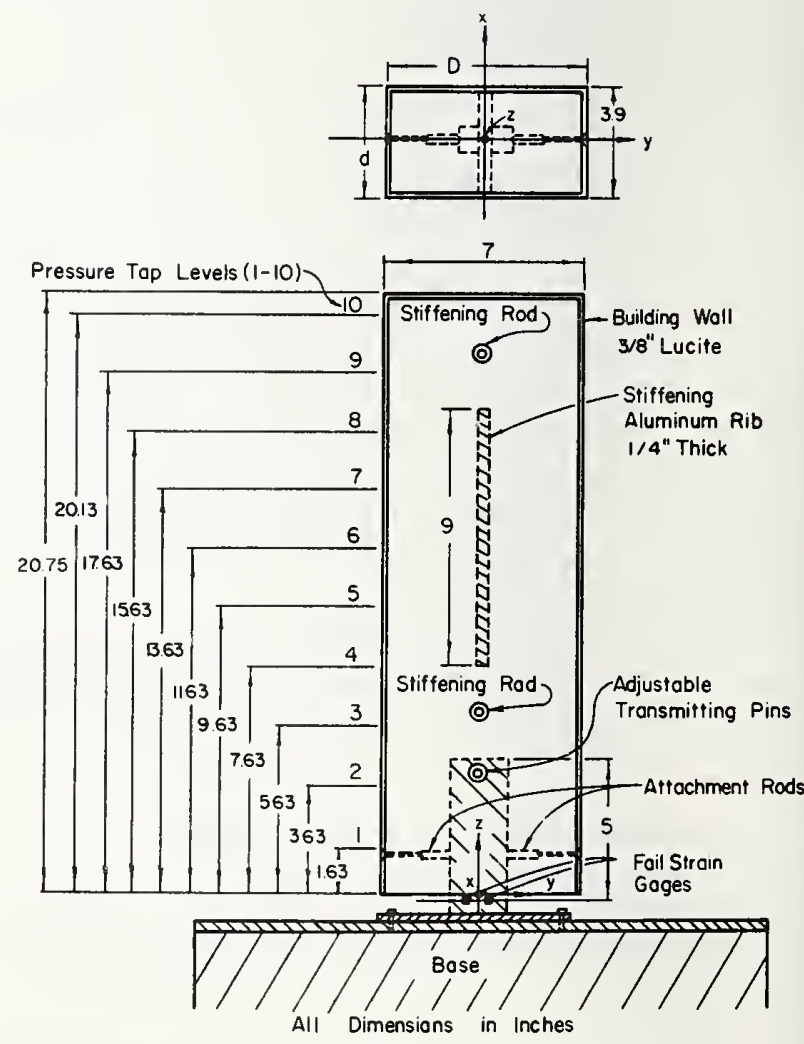

FIGURE 2. Sketch of the building model. 


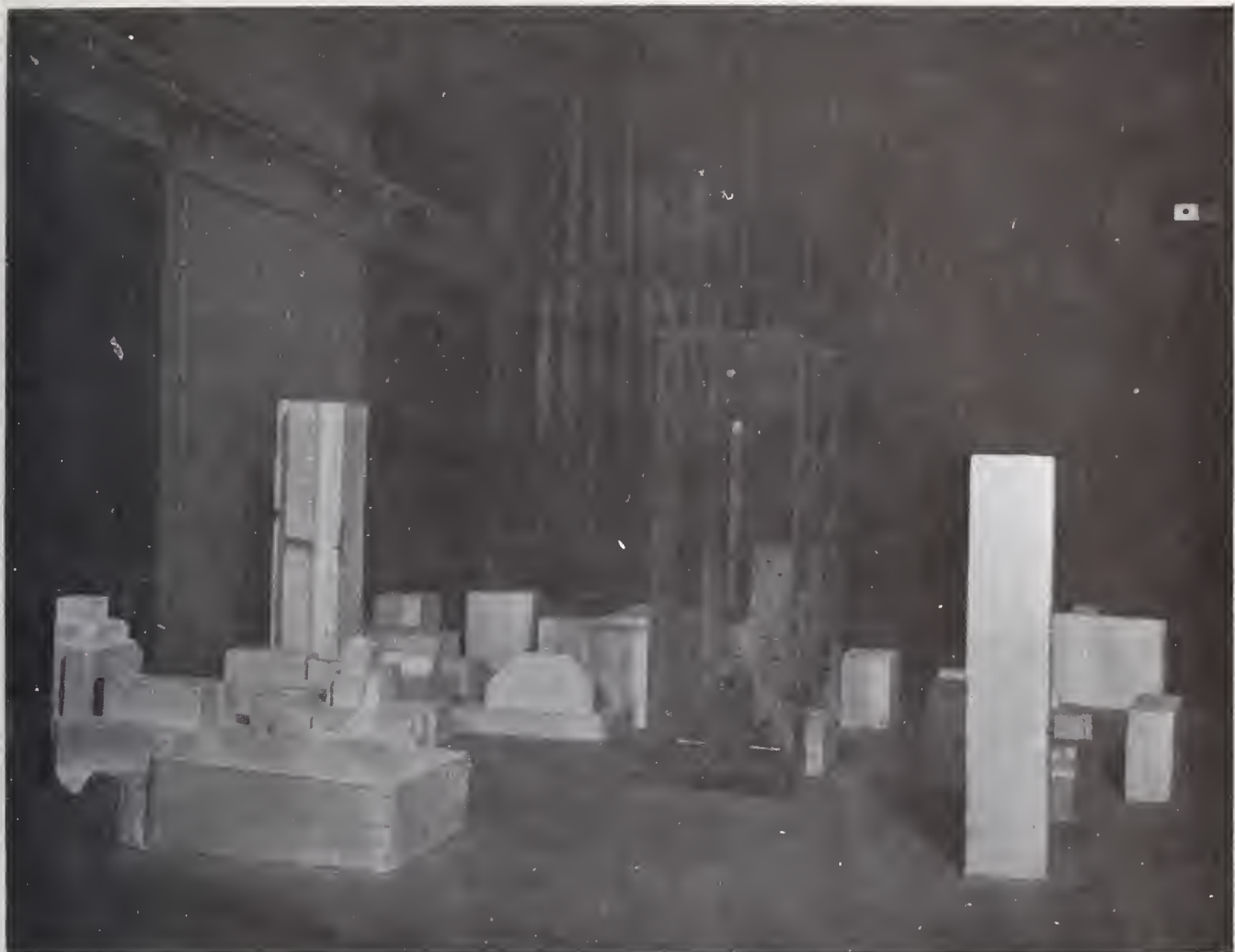

Figune 3. Overall view of the building model.

A sketch of the model is portrayed in Figure 2 which also shows the system of coordinates used and all important dimensions. On its faces 54 static pressure taps of 1/16-in. diameter were drilled. The taps were located as follows: 32 pressure taps on its wide face and 22 on its narrow face. The taps were bored only on two faces of the model. For measuring the pressure over the other two faces it was necessary to rotate the model by $180^{\circ}$. Furthermore, 13 pressure taps were also drilled on the building roof. Hence, it was possible to monitor the pressure at 121 locations for each building. The static pressure tap stations are also shown in Figure 2.

The surrounding buildings in the immediate vicinity were also simulated using a scale of $1: 38 t$. The former were constructed of styrafoam. Both the towers and these buildings were mounted on a rotatable plywood sheet base. For investigating the pressure-distribution dependence on the wind direc- tion it was possible to rotate the table $360^{\circ}$. The rotating table permitted visualization studies of the flow pattern for various wind directions. A photograph of this arrangement is provided by Figure 3 .

\subsection{Wind Tunnel}

The experimental investigation reported herein was conducted in the Colorado State University, Fluid Dynamics and Diffusion Laboratory, lowspeed wind tunnel. This tunnel is of closed circuit type and has a working section about $36.5 \mathrm{ft}$ long. Its axial-flow blower is driven by a $75 \mathrm{hp}$ constant speed motor. The fan is capable of generating air speeds up to $65 \mathrm{ft} / \mathrm{sec}$ in a $6 \times 6 \mathrm{ft}$ test section. The air speed can be changed continuously by varying the fan pitch. The model was placed at about $29 \mathrm{ft}$ downstream of the entrance section. Therefore, it was located in the thick boundary-layer region.

The removable side panels of the working section are made of glass in order to allow visualization 


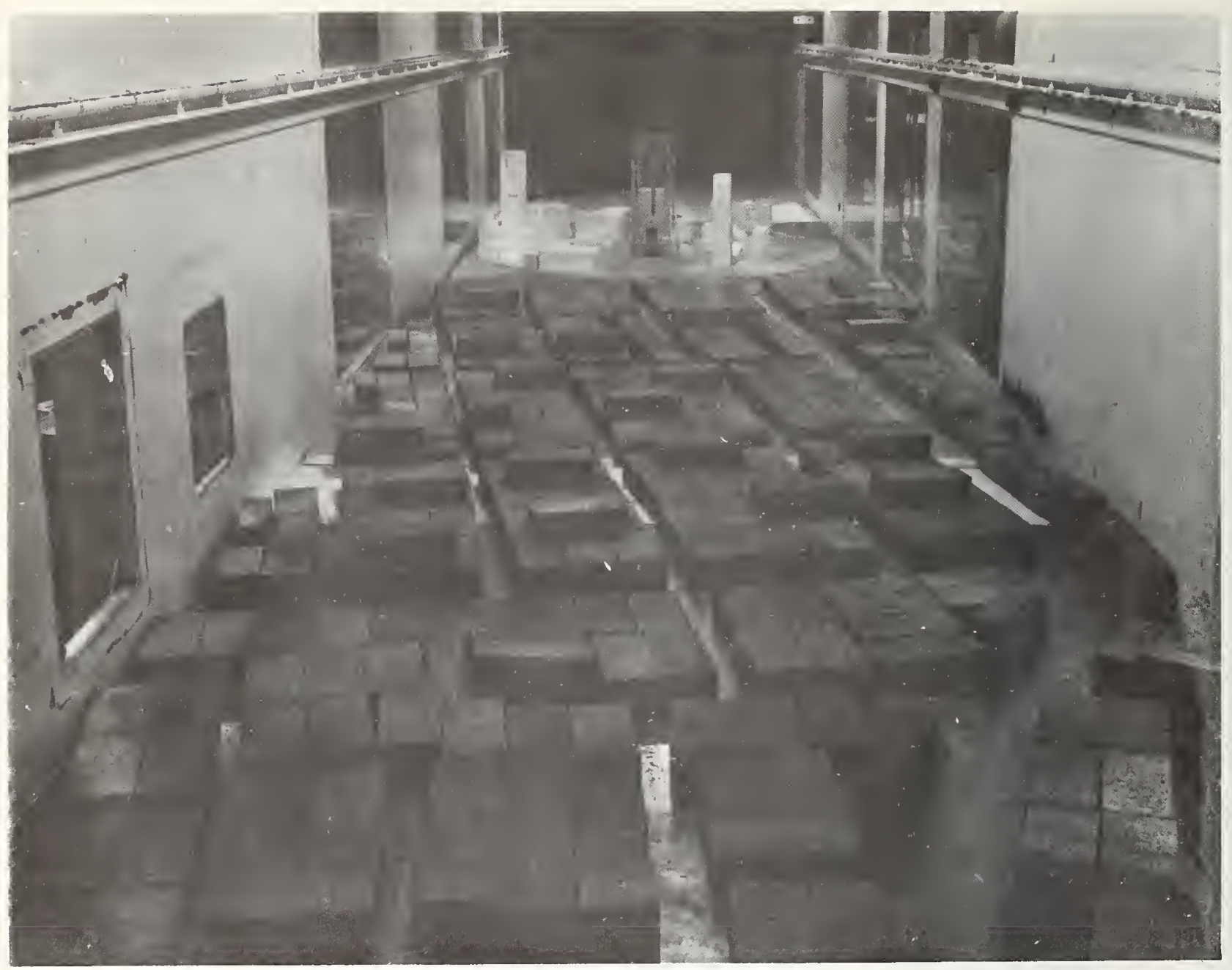

Figure 4. Overall view of the test section.

studies of the flow. Along the center line of the top panel a Pitot-static tube was inserted above the upstream building for continuous monitoring of the free-stream velocity, i.e., outside the boundary layer. Its location is shown in Figure 1.

An electrically operated traversing and positioning mechanism was used for continuous movement of the Pitot-static tube and/or hot-wire probe. This mechanism permitted fine control of position within $0.005 \mathrm{in}$. along lines parallel to the $x, y$ and $z$-axes.

\subsection{Upstream Conditions}

A complete simulation of the natural wind characteristics includes the generation of a suitably thick and turbulent boundary layer [1, 2]. Furthermore, the modeling of the upstream urban configuration is necessary for simulating the real flow conditions. As a result, the upstream urban topography was appropriately modeled using the same scale as for the building. This model extended over a distance of more than 1 mile (see Fig. 1) upstream of the studied buildings. It was simulated by covering the tunnel floor with an adequate configuration of modular bricks $\left(2 \frac{1}{4} \times 3 \frac{5}{8} \times 7 \frac{5}{8}\right.$ in. $)$. A photograph of the latter for the NE wind direction is shown in Figure 4. For every particular wind azimuth, i.e., free-stream velocity direction, an appropriate upstream roughness was employed.

Next, for generating a higher turbulence level, a vortex generator $[9]$ was installed at the entrance of the working section (see Fig. 1). This generator also caused an initial thickening of the boundary layer. For this purpose an asymmetric arrangement of two rows of modular briclis $1.3 \mathrm{ft}$ total height was used. Both the upstream roughness, i.e., the upstream modeled city, and the vortex generator caused the necessary thickening of the boundary layer and the desired turbulence intensity level. 


\section{Experimental Technique and and Instrumentation}

\subsection{Flow Visualization}

Two visualization methods were employed, paper tufts and thread tufts. The former, attached to wire grids and placed upstream and/or downstream of the buildings, were used to obtain a qualitative picture of the flow pattern for various wind directions.

The thread tufts were glued directly on the model faces. They permitted an acceptable qualitative indication of the flow pattern along the building faces to be obtained. As will be seen later both visualization methods established clearly the overall flow pattern and the existence of vortices downstream of the buildings. Moreover, the influence of the surrounding buildings on the flow was also visualized.

\subsection{Pressure and Velocity Measurement}

Average static pressures on the model faces were measured by means of an electronic pressure meter (Trans-Sonic, Type 120) with a resolution of $0.0001 \mathrm{~mm} \mathrm{Hg}$. The overall range of this manometer is $30 \mathrm{~mm} \mathrm{Hg}$.

A Pitot-static tube located $2.43 \mathrm{ft}$ above the model as shown in Figure 1 was employed to measure the static pressure and the mean velocity in the uniform flow. A Prandtl standard Pitot-static tube with a hemispherical impact head was utilized [10]. Its impact orifice is $1 / 32$-in. in diameter. This probe was also used to measure the velocity profile upstrcam of the building. The velocity change along the $z$-axis was measured at $1 \mathrm{ft}$ upstream of the model. In performing these measurements the Trans-Sonic pressure meter was utilized. Furthermore, the velocity change along the vertical direction was also recorded on an $x-y$ plotter (F. L. Moseley Co., Model 135). When the latter was carried out, the Pitot-static probe was moved continuously by means of the traversing mechanism.

The fluctuating pressures, rms and instantaneous peak pressure, on the model faces were measured at all the pressure taps by means of low-pressurc differential pressure transducers (Statham, Model PM 283). Six similar transducers were utilized. These transducers were installed inside the model and were connected closely to the pressure taps. The transducers with the associated tubing ( $\frac{3}{8}$-in. I.D. vinyl tubing) had a frequency response larger than about $200 \mathrm{~Hz}$. The transducers exhibited a reasonable linear calibration curve. The latter was carried out utilizing a precise micromanometer (The Meriam Instrumentation Co., Model 34FB2). The pressure differ- ence was measured with respect to the static pressure of the uniform free-stream. The latter was obtained from the Pitot-static tube located above the model (see Fig. 1). The reproducibility of the calibration curves was within $1 \%$ to $3 \%$. In comnection with these measurements the following auxiliary equipment was used:

(1) A variable range amplifier (Dana, Model 3500 ). Usually, an amplification of 200 was utilized;

(2) A seven channel tape-recorded (Mincom, Type 100) for recording and storing the amplified signal for further analysis;

(3) An electronic voltmeter (Bruel \& Kjaer, Type 2416) for measurement of $\mathrm{rms}$ and peak values;

(4) A recording wave analyzer (General Radio, Recording Sound and Vibration Analyzer, Type 1911-A) for frequency spectra measurement;

(5) A dual-beam oscilloscopc (Tektronix Storage Oscilloscope, Type 564) for quick assessment of the output signal pattern;

(6) A Polaroid camera (Type C-12) for taking oscillograms of the output signal;

(7) A digital DC voltmeter (Hewlett-Packard, Model 3440 A) for monitoring various output signals.

A simplified block diagram of this system is shown in Figure 5 and, a general view of the auxiliary equipment is provided by Figure 6 .

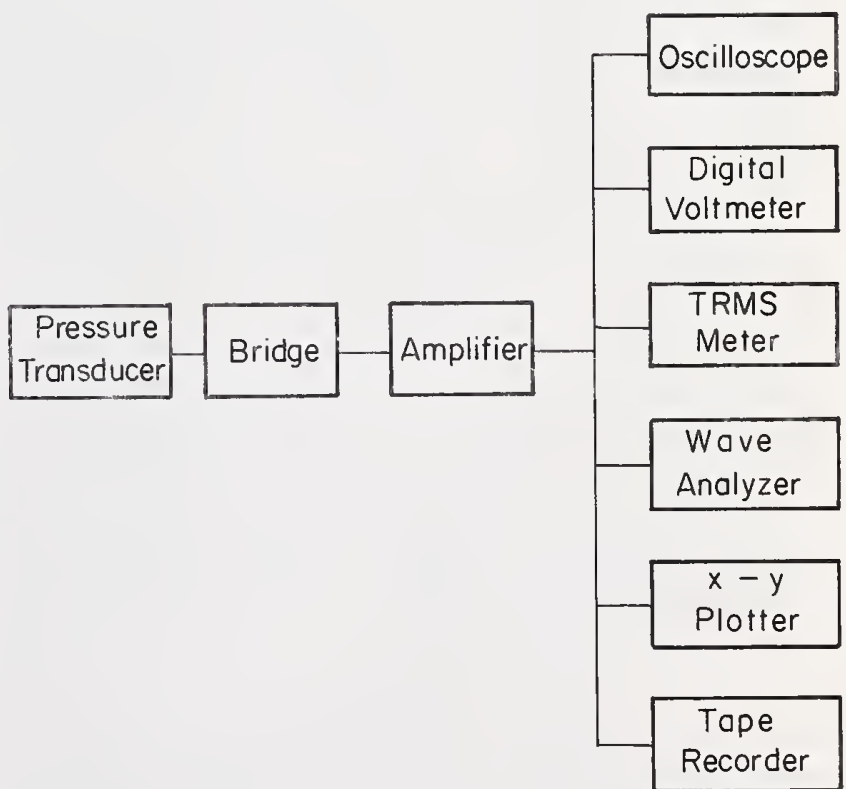

Figure 5. Simplified block diagram of the pressure-transducer system. 


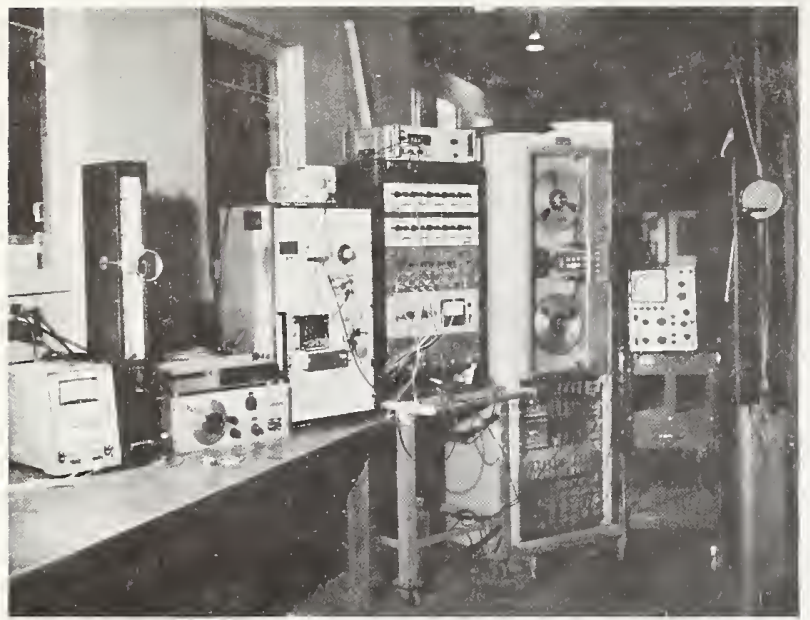

Figure 6. General view of the equipment.

\subsection{Turbulence Intensity Measurement}

The longitudinal turbulence intensity was measured upstream of the model by means of a hot-wire anemometer. The measurements were carried out at the same distance from the model as for the mean velocity, i.e., at $1 \mathrm{ft}$ upstream of the model, along the z-axis. When performing these measurements the hot-wire probe was positioned by means of the traversing mechanism. The hot-wire anemometer unit used in the present experiment is a constanttemperature fully transistorized system conceived, designed and built at the Fluid Dynamics and Diffusion Laboratory. Its output noise level is less than about $200 \mu V$ and its frequency response is beyond $100 \mathrm{KHz}$. A detailed report about this unit, its characteristics and performances, is under preparation. A copper-plated tungsten wire of 0.00025 -in nominal diameter and 0.06-in effective length $(1 / d=240)$ was employed.

The hot-wire calibration was effected by locating it in the free-stream above the model. It was carried out employing a Pitot-static tube. The calibration curves obtained revealed that the so-called Iing's law [11], i.e., the $\frac{1}{2}$ power linear relation $\left(E^{2} \propto \sqrt{U}\right)$ was satisfied reasonably well for the velocity range of interest. The latter ranged from about 10 to 60 $\mathrm{ft} / \mathrm{sec}$. It was reproducible within about $3 \%$. The turbulence intensity, which is commonly defined as $u_{\mathrm{rms}} / \bar{U}$, is given by [12]

$$
\frac{u_{\mathrm{rms}}}{\bar{U}}=\frac{4 \bar{E}^{2}}{\bar{E}^{2}-E_{0}^{2}} \frac{e_{\mathrm{rms}}}{\bar{E}},
$$

where the subscript rms denotes square-root of mean (time-averaged) square values, i.e., $\sqrt{\overline{u^{2}}}$ and $\sqrt{\overline{e^{2}}}$, and, the over bar denotes time-averaged (or mean values). In the above relationship the mean velocity is designated by $\bar{U}$ and $\bar{E}$ stands for the time-averaged voltage drop across the wire, i.e., the DC voltage necessary to balance the bridge under steady conditions. The fluctuating velocity is denoted by $u$ and the corresponding instantaneous voltage drop by $e$, i.e., the instantaneous $\mathrm{AC}$ voltage. The voltage drop across the wire in still air (zero velocity or shielded hot-wire) is denoted by $E_{0}$. It is constant for a chosen resistance ratio (overheating ratio). In connection with the hot-wire unit, a true RMS meter, a digital DC voltmeter and a dual-beam oscilloscope were used. This equipment is described in Section 3.2.

\subsection{Moment Measurements}

As mentioned earlier, the direct measurement of the total overturning moment, i.e., mean and fluctuating, was particularly stressed. In order to eliminate all the other force components a suitable strain-gage dynamometer system was conceived and built. Hence, the model was mounted in the wind tunnel by attaching it rigidly to an aluminum beam $(2 \times 1 \times 5$ in. $)$ by means of four rods $90^{\circ}$ apart. In turn, the beam was rigidly anchored to a massive base located underneath the wind tunnel floor. Thus, the dynamometer-building model system constituted a cantelever beam with end-load. The aerodynamic force acting on the model building was transmitted to the beam by two adjustable pins $180^{\circ}$ apart. The pins were positioned close to the beam's free-end and they were tightened manually to the former. At the lower end of the beam four foil strain gages (Micro-Measurement, Type EDDY-250B6-350) were cemented. They constituted the four arms of a Wheatstone bridge. Thus, the overall sensitivity of the strain-gage system was increased four times. This balance was sensitive only to the overturning moment about the weak axis of the building. The response of the strain-gage for any lateral load was about 20 times smaller than for the frontal force producing the overturning moment. In order to stiffen the model and to increase its natural frequency an aluminum rib and stiffening rods were mounted rigidly between its two wider faces. The balance system is portrayed in Figure 2 .

For obtaining reliable and dependable measurements of the moment due only to aerodynamic forces in a stationary structure the natural frequency of the entire aeroelastic system, i.e., the beam and the building model, should be larger than any forcing frequency expected. The pressure survey revealed that the maximum frequency of interest was smaller 
than about $200 \mathrm{~Hz}$. Consequently, the balance system was conceived such that its natural frequency was $200 \mathrm{~Hz}$. The strain for a moment of about 25 lb-in. was of the order of $6 \mu \mathrm{in} / \mathrm{in}$.

As a result, a relatively stiff model was obtained. For a free-stream velocity of $50 \mathrm{fps}$ the reduced velocity [2] $U_{\infty} / D n$ was about 0.43 . In the latter, $d$ is the largest dimension of the model cross-section and $n$ is the natural frequency of the dynamometer system. Thus, the tip deflections of the model were negligible.

A simplified block diagram of the moment measuring system is provided by Figure 7. Essentially the same auxiliary equipment as employed for the pressure measuring system was utilized. The excitation and balance network of the strain-gage is also shown in Figure 7.

The balance was calibrated by applying various forces at different heights along the building. A sample of the kind of calibration curves obtained is shown in Figure 8. A satisfactory linear change was obtained. The reproducibility of the calibration curves was within $2 \%$ to $5 \%$.
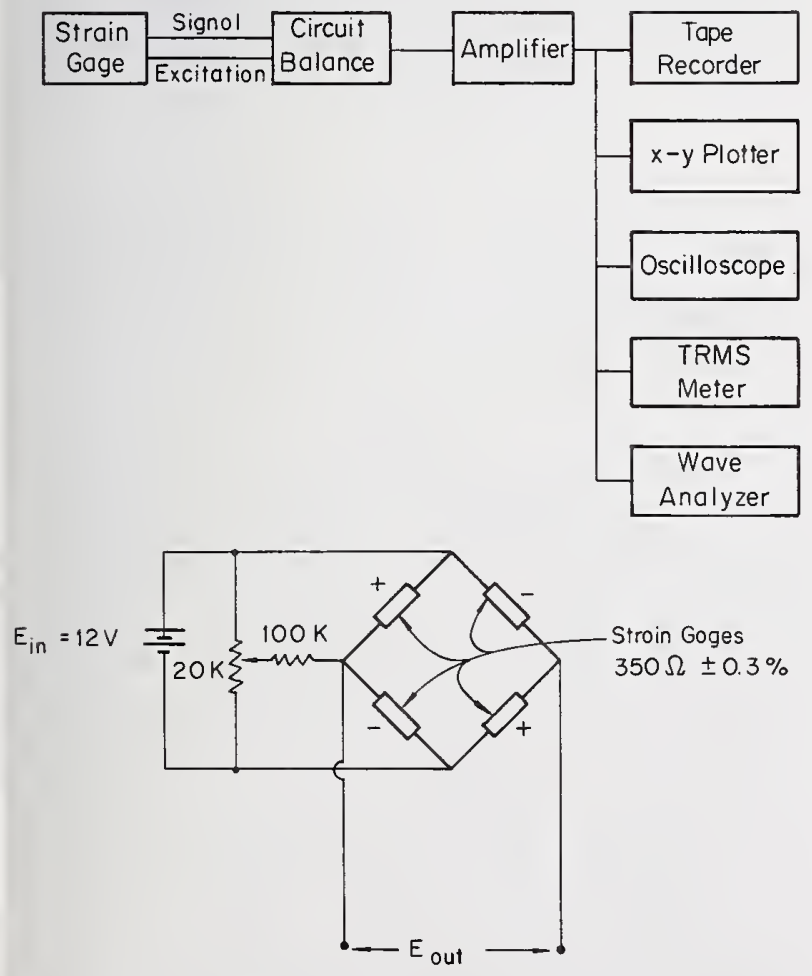

Figure 7. Simplified block diagram of the moment measuring system.

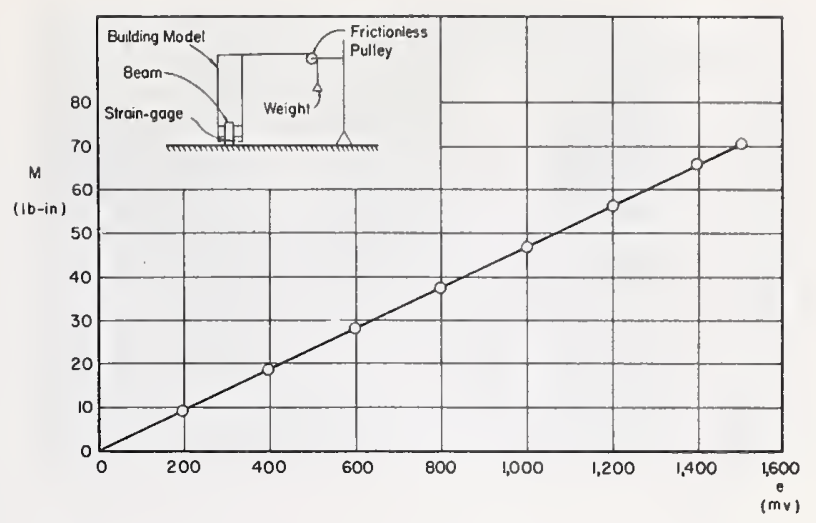

Figure 8. Typieal ealibration eurve of the balanee.

\section{Results}

The measurements were carried out for three different wind directions (free-stream velocity directions)-NE, N and NIV. This was achieved by suitable rotation of the turnable base. However, only the results for the NE direction are presented with emphasis on overturning moment of the upwind building. No significant basic changes, except for the absolute value of the pressure distribution, were obtained for the other two directions. Moreover, it was found that this particular direction was the most suited for performing the direct measurement of the overturning moment. This choice was determined by structural consideration of the aerodynamic balance design.

The system of coordinates used in the presentation of the results is portrayed in Figures 1 and 2. For generality, the results are presented in dimensionless form. Dimensional variables, wherever employed, are denoted by an asterisk. As the experimental results are presented below, some pertinent discussions are interspersed wherever it is deemed helpful for the proper interpretation of the results.

\subsection{Establishment of the Flow}

An extensive series of flow visualization trials were carried out for the purpose of studying the overall flow pattern upstream and, principally, downstream of the building. Visualization of the flow by means of paper tufts gave a clear picture of the flow pattern. Particularly, the tufts indicated the flow within the wake. Its strongly turbulent character and its vortex structure were clearly observed. The wake extended vertically above the model up to about $10 \%$ of its height. The longitudinal extent of the wake was not evaluated due to the interference of the two model buildings. The 

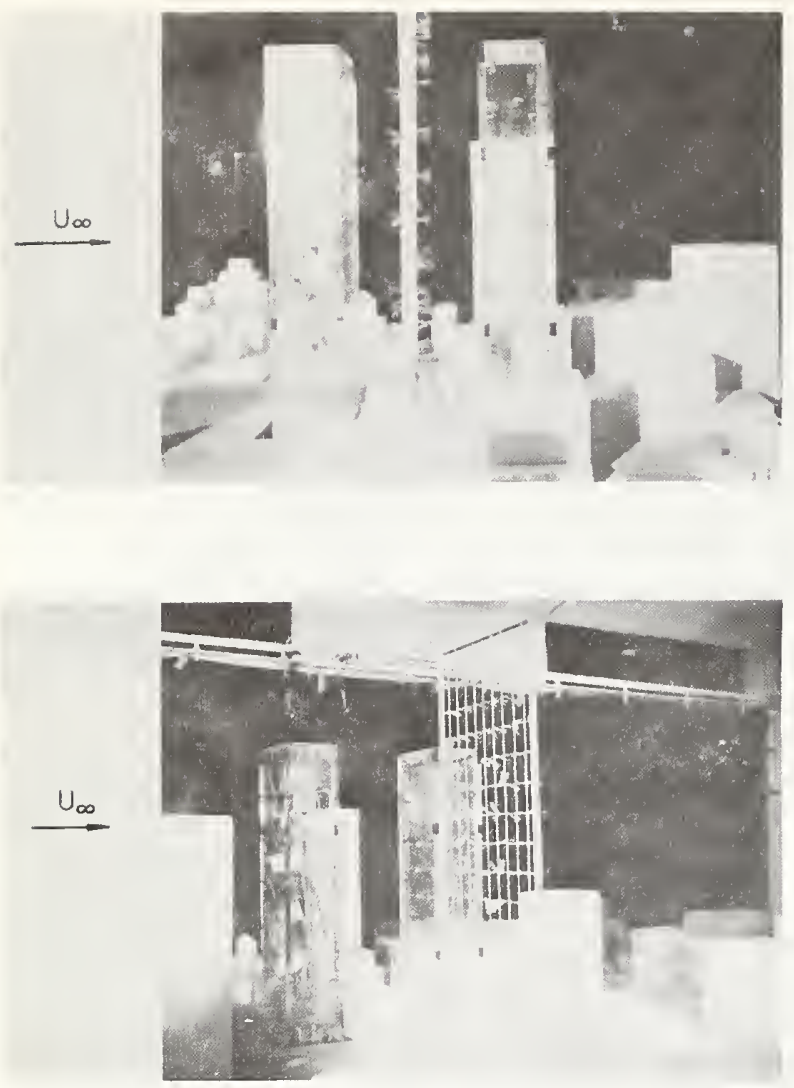

Figure 9. Visualization of the flow by means of paper tufts.

second tower was situated in the wake of the front building. The vertical extent was similar for both buildings. Figure 9 is a photograph of paper tufts showing the wake.

The thread tufts glued to the model walls showed distinctly the flow pattern along them. Generally, a down draft was discerned along the front wall. Near its bottom a clear rotational trend was observed. On the other hand, along the leewind wall an updraft and a rather turbulent rotational pattern were indicated by the tufts. Photographs of these patterns are shown in Figure 10. A similar flow pattern is reported in Reference $S$.

\subsection{Mean Velocity Survey}

The wind velocity gradient upstream of the building is of utmost importance in determining the flow characteristics. Its variation with height depends on the particular configuration of the roughness structure [13]. Furthermore, for finding the Reynoldsnumber dependence, the velocity gradient and the resulting average pressure distribution should be practically independent of the upstream velocity variation.

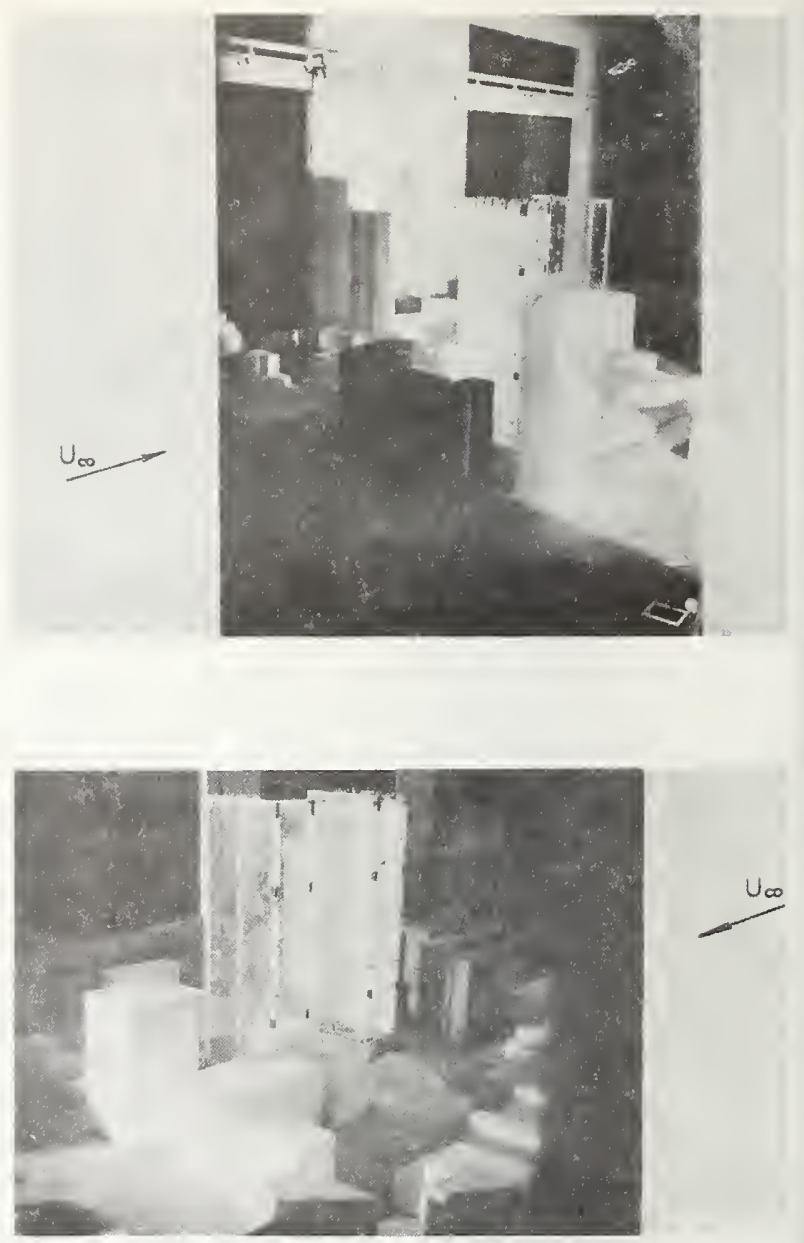

FIGURE 10. Visualization of the flow pattern along the model walls by means of thread tufts.

Generally, the mean velocity profile over terrains of differing roughness configuration is given either by a logarithmic law or a power law [13]. The latter may be described by

$$
U^{*} \propto z^{* \alpha},
$$

where $z^{*}$ denotes the height and the value of the exponent $\alpha$ depends on the particular roughness structure. This representation was employed in the present work.

The mean velocity variation along the $z$ direction was measured upstream of the model, i.e., at $1 \mathrm{ft}$ upstream of the building model, at more than 15 stations over a distance of about 55 in. Concurrently, it was also recorded for continuous traverses along the $z$ axis. All the measurements were performed at a uniform free-stream velocity of $50 \mathrm{ft} / \mathrm{sec}$. At this velocity, denoted by $U_{\infty}{ }^{*}$, a sufficiently thick boundary layer at the building model location could be obtained. The Reynolds number based on the 
largest dimension of the cross-section was about 182,000 .

Next, the mean velocity was measured for various upstream conditions. This investigation was performed for verifying the adequacy of the atmospheric surface-layer flow simulation in the wind tunnel. The measurements were carried out for the following cases: (1) clear wind tunnel; (2) vortex generator installed; (3) vortex generator and upstream roughness, i.e., the scaled model of the upstream urban configuration, installed (real flow conditions). In all these three cases the scaled model of the building and of the adjacent buildings were located in the wind tunnel (see Fig. 1). It was found that the velocity change is strongly affected by the upstream conditions. The power-law variation was satisfied for all the cases, but the value of the exponent differed for each case.

Hereafter, all the results are presented in dimensionless form. The dimensionless coordinates are defined by

$$
x, y, z=x^{*} / h, \quad y^{*} / h, \quad z^{*} / h,
$$

where $h$ is the building height, and the dimensionless velocity by

$$
\mathbf{H}=\mathbf{H} / U_{1}{ }^{*} \text {, }
$$

where $U_{1}{ }^{*}$ is the mean velocity at $z^{*}=h$. Hence, the dimensionless mean velocity is given by

$$
U=z^{\alpha} \text {. }
$$

The measured mean velocity distributions along the $z$ direction for the above three cases are displayed in Figure 11. The results were reproducible within $3 \%$ to $5 \%$. The field wind profile is also shown in Figure 11 (case 4). The latter is based on 100-year wind data at $250 \mathrm{ft}$ height above the surface. For obtaining the wind variation a $\frac{1}{7}$-power law was utilized for selected heights between 250 and 1,000 ft. On the other hand, a $\frac{1}{3}$-power law was employed for heights below $250 \mathrm{ft}$ [14].

For the sake of comparison the results for all the three cases and the field wind (case 4) are summarized below:

$\begin{array}{ccc}\text { Case } & U_{\mathbf{L}^{*}}(\text { feet/sec }) & \alpha \\ 1 \ldots \ldots \ldots- & 47 & 0.107 \\ 2 \ldots \ldots \ldots- & 45 & 0.206 \\ 3 \ldots \ldots \ldots- & 45 & 0.446 \\ 4 \ldots \ldots & 96.8 & 0.157\end{array}$

Note that $h=20.75$ in. for cases (1), (2) and (3) whereas $h=666$ feet for case (4). The values of the exponent are in general agreement with repre-

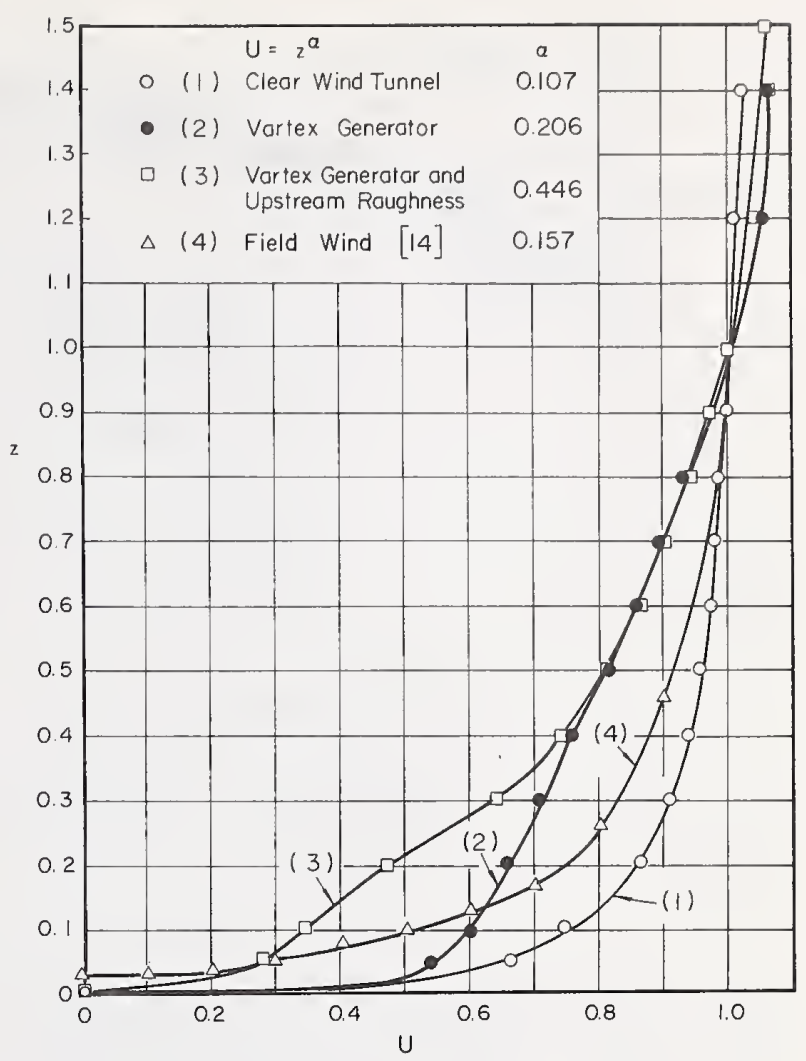

Figure 11. Mean velocity profile upstream of the model.

sentative values reported by Davenport [13]. The influence of the upstream conditions, i.e., vortex generator and/or upstream roughness is easily observed. Thus, the atmospheric surface-layer flow simulation was acceptable.

In the following, the results for case (3) are presented. This is the case of interest since the upstream urban configuration was appropriately modeled, i.e., the case of the real flow conditions.

As mentioned earlier, a suitably thick boundary layer is desired for a meaningful simulation of atmospheric-surface-layer conditions. Consequently, it was important to find out the vertical extent of the boundary layer immediately upstream of the model. The boundary-layer thickness was defined, as is commonly done, as the distance from the wind tunnel floor where $U^{*} / U_{\infty}{ }^{*}=0.99$. The recorded velocity profile, i.e., dynamic head, at $1 \mathrm{ft}$ upstream of the model is displayed in Figure 12. This record was obtained by continuously moving the Pitotstatic tube using the traversing mechanism. The probe was moved at a sufficiently small speed to allow a suitable response. A boundary-layer thickness of about $49.7 \mathrm{in}$. was measured. Thus, the dimensionless boundary-layer thickness $\delta^{*} / h=2.4$. This corresponds to a real boundary layer about $1,600 \mathrm{ft}$ thick. 


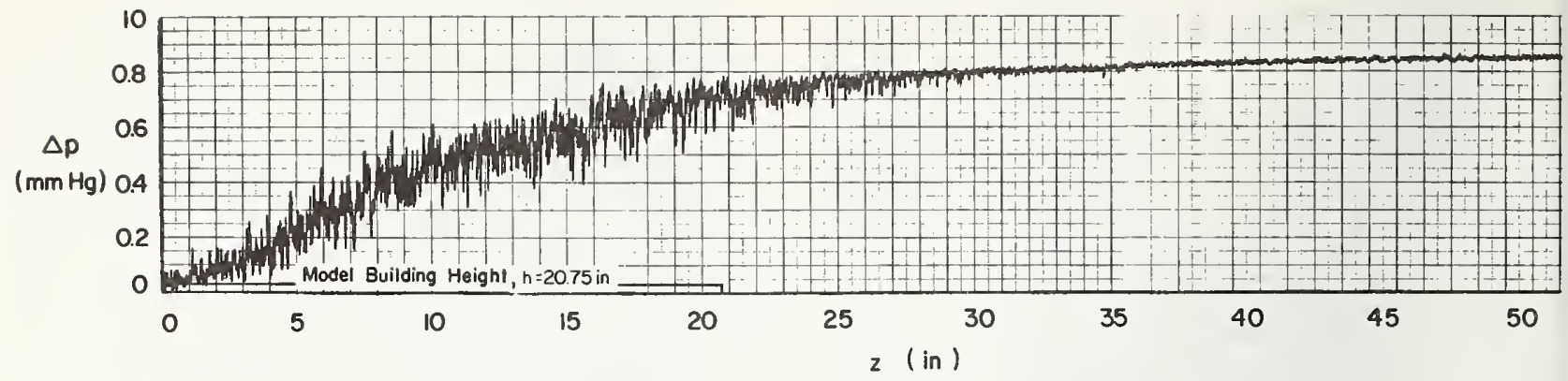

Figure 12. Record of velocity profile upstrcam of the model.

As a result, a sufficiently thick boundary layer was obtained. Hence, the simulation conditions were fulfilled.

\section{3. 'Turbulence Intensity Measurement}

Simultaneously with mean velocity measurements the turbulence intensity based on local mean velocity,

$$
T u_{x}=\frac{u_{\mathrm{rms}}(z)}{\bar{U}(z)}
$$

was monitored. In this relation $u$ is the fluctuating velocity parallel to the mean flow velocity $\bar{U}$. The subscript rms denotes square-root of mean (timeaveraged) square value, i.e., $\sqrt{\bar{u}^{2}}$.

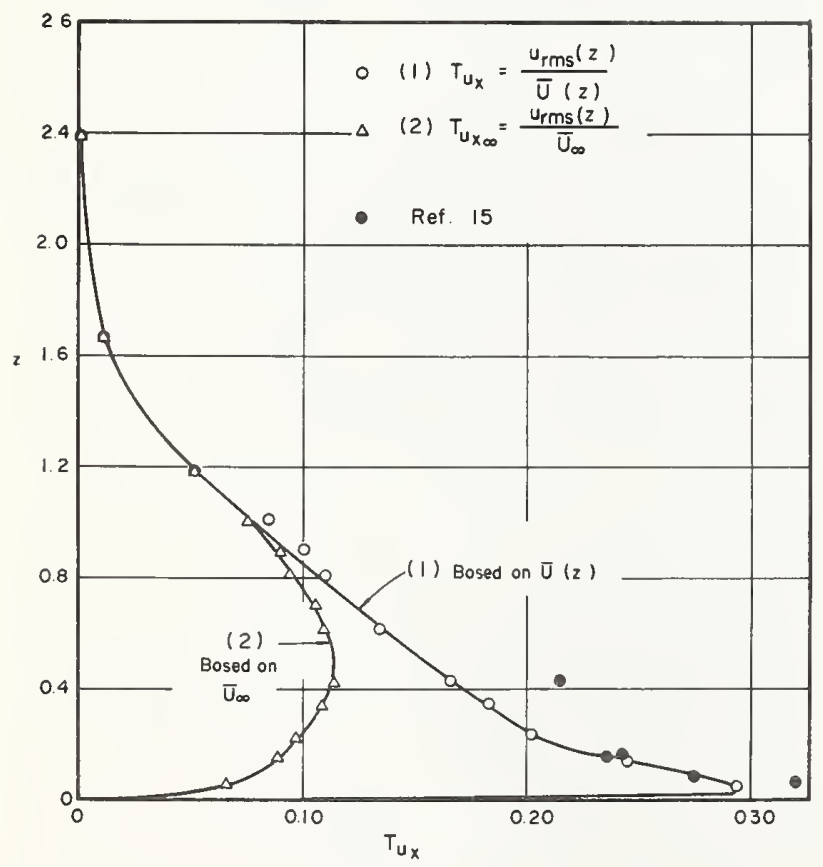

Figure 13. Turbulence intensity variation upstream of the model.
The results for case (3) are displayed in Figure 13. They were reproducible within 2 to $3 \%$. Both the turbulence intensity based on local and on freestream mean velocity, i.e., based on $\bar{U}(z)$ and $\bar{U}_{\infty}$, are shown. Based on the local mean velocity a maximum turbulence intensity of about $29 \%$ was measured at $z=0.05$. When it was based on the freestream velocity a maximum of about $12.5 \%$ was monitored at $z=0.4$. The different location of the maximum is caused by the local mean velocity variation. As the free-stream region is approached the difference between them decreases. It can be observed that, at a distance of about $0.2 \mathrm{~h}$ above the building model, they are practically equal. The turbulence intensity at the outer edge of the boundary layer, i.e., at $z=2.4$, was about $0.2 \%$. The latter is practically the free-stream turbulence intensity. The relatively high turbulence level throughout the boundary layer was caused by the upstream conditions, i.e., vortex generator and upstream roughness. The high level of turbulence within the boundary layer can also be observed from the recorded velocity profile along the $z$-axis. The latter is portrayed in Figure 12.

Unfortunately, no field data are available for comparing the turbulence intensity distribution. On the other hand, the results exhibit a reasonable agreement with the measurements in the lowest atmosphere reported by Singer [15].

\subsection{Pressure Survey}

The aerodynamics forces and moments acting on a structure are determined by the wind characteristics. Due to the velocity gradient and the turbulence structure within the atmospheric surface layer (the boundary layer) both mean and fluctuating forces are of importance in finding the structural response to wind loading. Furthermore, the local instantaneous peak force together with the fluctuating force is also important in designing outer skin panelling and 
window glass. Knowledge of the pressure distribution, i.e., mean, fluctuating and peak pressure, permits computation of these forces.

A detailed survey of the pressure distribution along the building model was carried out. The local pressure was measured with respect to the static pressure of the uniform free-stream above the model, i.e., the static pressure outside the boundary layer. The latter was monitored by means of a Pitot-static tube located as shown in Figure 1.

The total local pressure at any point along the model wall is

$$
p-p_{\infty}=\left(\bar{p}+p^{\prime}\right)-p_{\infty},
$$

where $p$ is the total local pressure, $\bar{p}$ the local mean pressure, $p^{\prime}$ the local fluctuating pressure and $p_{\infty}$ is the free-stream static pressure. The overbar denotes time-averaged (or mean) values. Next, Eq. (7) can be written

$$
\Delta p=\Delta \bar{p}+p^{\prime}
$$

where

$$
\Delta \bar{p}=\bar{p}-p_{\infty} .
$$

The fluctuations of the free-stream static pressure are completely negligible with respect to the local fluctuating pressure. Then, by taking the meansquare of the total local pressure we obtain

$$
\overline{\Delta p^{2}}=\overline{\Delta p^{2}}+\overline{p^{\prime 2}} \text {. }
$$

Next, for incompressible flow at small velocities the local mean pressure coefficient is defined by

$$
C_{p}=\frac{\overline{\Delta p}}{\frac{1}{2} \rho U_{\infty}{ }^{* 2}},
$$

whereas the local fluctuating pressure coefficient

$$
C_{p_{f}}=\frac{p_{\mathrm{rms}}}{\frac{1}{2} \rho U_{\infty}^{* 2}}
$$

where $p_{\text {rms }}$ denotes square-root of mean (time-

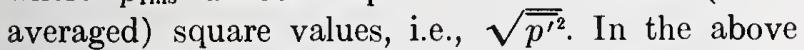
two relationships $U_{\infty}^{*}$ represents the free-stream velocity above the model and $\rho$ is the air density. In a similar fashion the local instantaneous peak pressure coefficient is

$$
C_{p_{\max }}=\frac{p_{\max }^{\prime}}{\frac{1}{2} \rho U_{\infty}^{* 2}}
$$

where $p_{\text {max }}^{\prime}$ is $\frac{1}{2}$ of instantaneous peak-to-peak pressure fluctuation. In terms of the mean pressure coefficient, the local fluctuating and peak pressure coefficients are

$$
\begin{aligned}
C_{p_{f}} & =C_{p} \frac{p_{\mathrm{rms}}}{\overline{\Delta p}}, \\
C_{p_{\max }} & =C_{p} \frac{p_{\max }^{\prime}}{\overline{\Delta p}} .
\end{aligned}
$$

In order to test the variation in time of the pressure fluctuations at every position on the model, the pressure transducer signal was monitored on an oscilloscope over a period of several minutes. The observed changes were completely negligible. A typical oscillogram of the transducer output signal is displayed in Figure 14.

As mentioned earlier, the results reported herein were obtained for $U_{\infty}{ }^{*}=50 \mathrm{ft} / \mathrm{sec}$ and for a NE wind. Consequently, the flow had an incident angle of $6^{\circ}$ with respect to the $x$-axis (see Fig. 15). For purposes of calculating the mean force the flow can be considered normal to the wider face of the model. The mean pressure coefficient distribution and the isobars of the ratio of the fluctuating and the peak pressure coefficient to the former on the front face of the model are shown in Figure 15. On the leewind face negative mean pressure coefficients were measured. Generally, the fluctuating pressure coefficient (rms) is smaller than the mean coefficient. Only near the model base is the former larger or of the same order as the latter. Within this domain the mean pressure is relatively small. On the other hand, over most of the face, the peak pressure is larger than the mean pressure. Peak pressures as large as 4 times the mean pressure were measured. It is, further, important to notice that the maximum peak

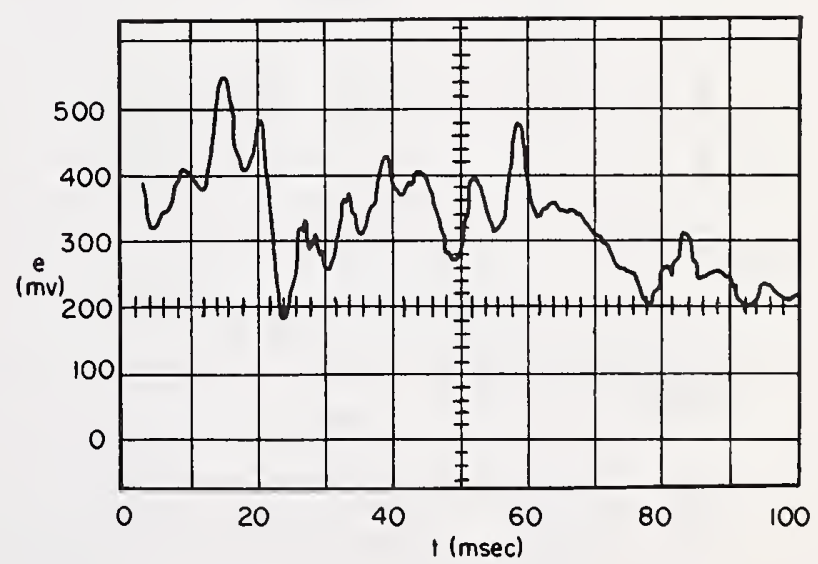

Figure 14. Oscillogram of the fluctuating pressure at $x=0.07, \quad y=0.17, \quad z=0.75, \quad\left(x^{*}=1.45 \mathrm{in}\right.$, $y^{*}=3.5$ in., $z^{*}=15.6 \mathrm{in}$.) ; sweep $10 \mathrm{msec} / \mathrm{cm}$, sensitivity $100 \mathrm{mv} / \mathrm{cm}$. 


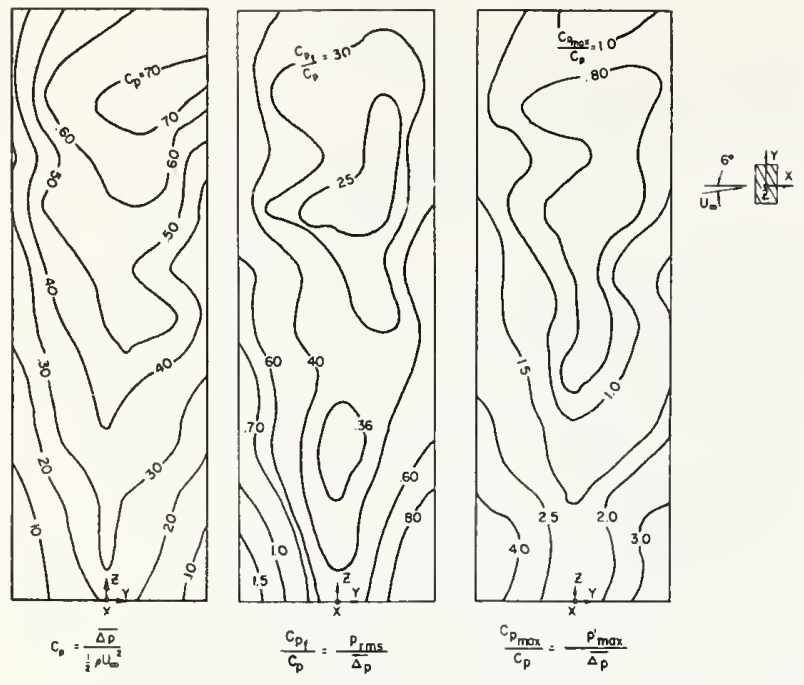

FIgURe 15. Mean, fluctuating and peak pressure coefficient distribution on the front face.

pressure occurred within regions where the fluctuating pressure was the largest. The large increase in both fluctuating and peak pressure near the building base may be attributed to the upstream roughness and the concomittant large turbulence intensity.

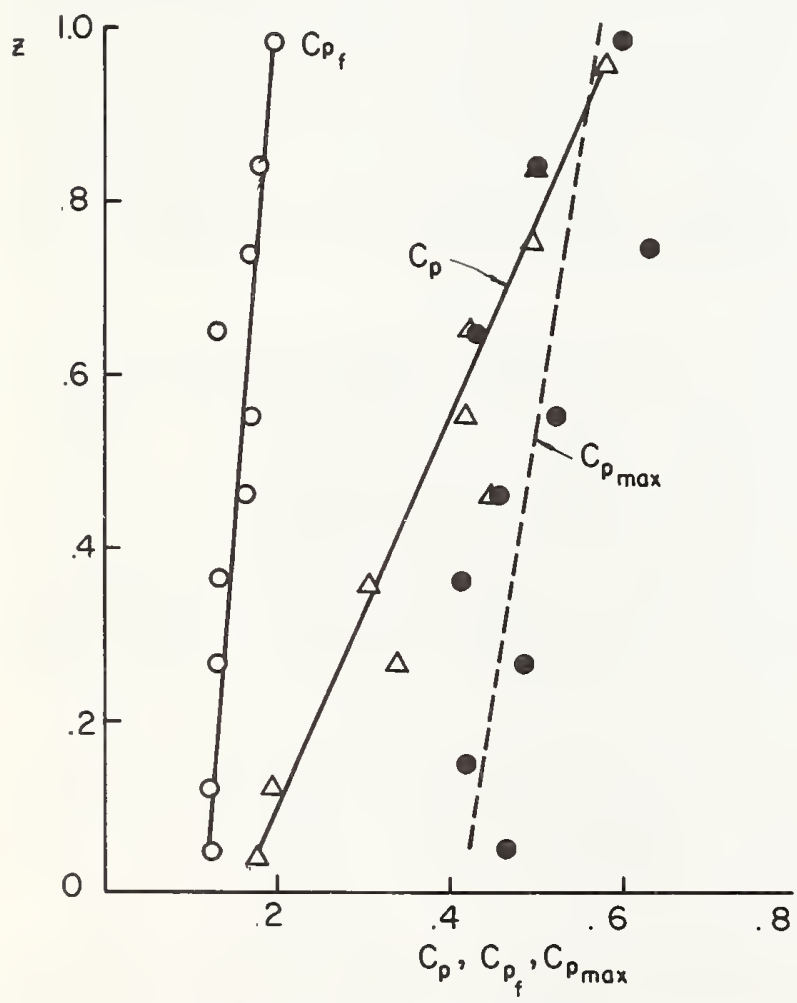

Figure 16. Variation of average mean, fluctuating and peak pressure cocfficicnt as a function of height.
For each cross-wise area element (area element normal to the mean velocity) it was possible to compute an average pressure coefficient, i.e., pressure coefficient per unit area. The change of the average mean, fluctuating and peak pressure coefficient with height is depicted in Figure 16. The mean and fluctuating pressure exhibit a linear variation while the peak pressure reveals a periodical change.

Simultaneously with pressure measurements a survey of pressure fluctuating-energy was carried out. The frequency spectrum at each station was recorded by a wave analyzer. The signal to noise ratio $(S / N)$ was larger than 10 for all the transducers employed. A characteristic frequency spectrum is shown in Figure 17. It was taken at the same station as the oscillogram displayed in Figure 14. Most of the pressure fluctuating-energy was concentrated at low frequencies. No significant contributions

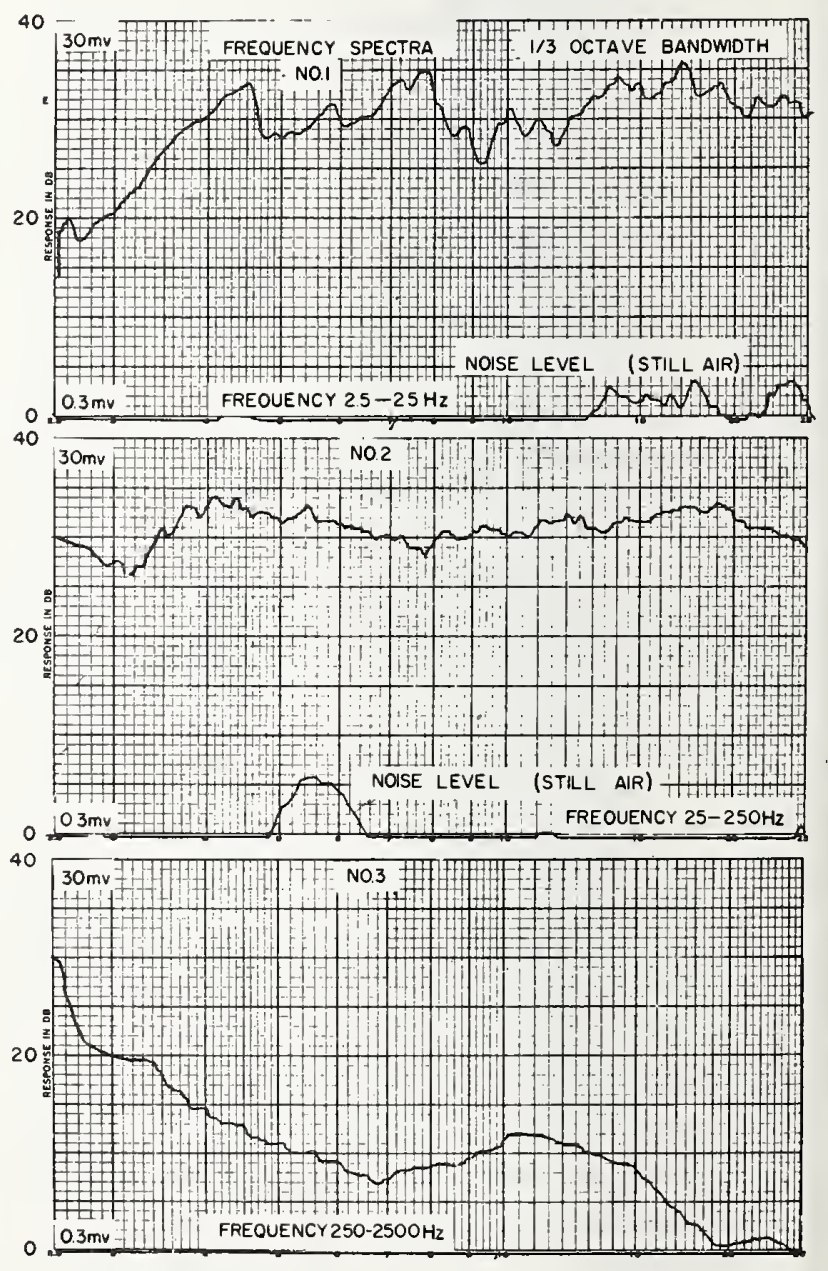

Figure 17. Typical frequency spcctrum of pressure fluctuating-energy; $x=0.07, y=0.17, z=0.75, \quad\left(x^{*}=\right.$ 1.45 in., $y^{*}=3.5$ in., $z^{*}=15.6$ in.). 
were obtained at frequencies larger than about 200 to $250 \mathrm{~Hz}$. A predominant frequency of about 17 $\mathrm{Hz}$ was observed. On the other hand, the oscillogram indicated a predominant frequency of about $20 \mathrm{~Hz}$. A report on the pressure fluctuating-energy survey is under preparation.

It was important to check the pressure distribution invariance with Reynolds number. For this purpose the mean pressure coefficient was measured for two different upstream velocities, i.e., for $U_{\infty}{ }^{*}=30$ and $50 \mathrm{feet} / \mathrm{sec}$, while the other conditions were unchanged. Then, a normalized pressure coefficient difference

$$
\gamma=\left|\frac{C_{p}(50)-C_{p}(30)}{C_{p}(50)}\right|,
$$

was computed at about 97 sample points. In Figure 18 the number of points for a constant value of $\gamma$ are displayed. At 77 sample stations $\gamma<0.2$. Furthermore, at 41 locations $\gamma=0.025$. On the other hand, only at 1 sample station $\gamma>0.8$. Hence, at most of the stations the change in pressure coefficient is negligible.

\subsection{Moment Measurement}

The direct measurement of the total overturning moment was particularly emphasized. The main objective of this investigation was to obtain an accurate measurement of the fluctuating moment. As mentioned earlier, the local fluctuating and peak

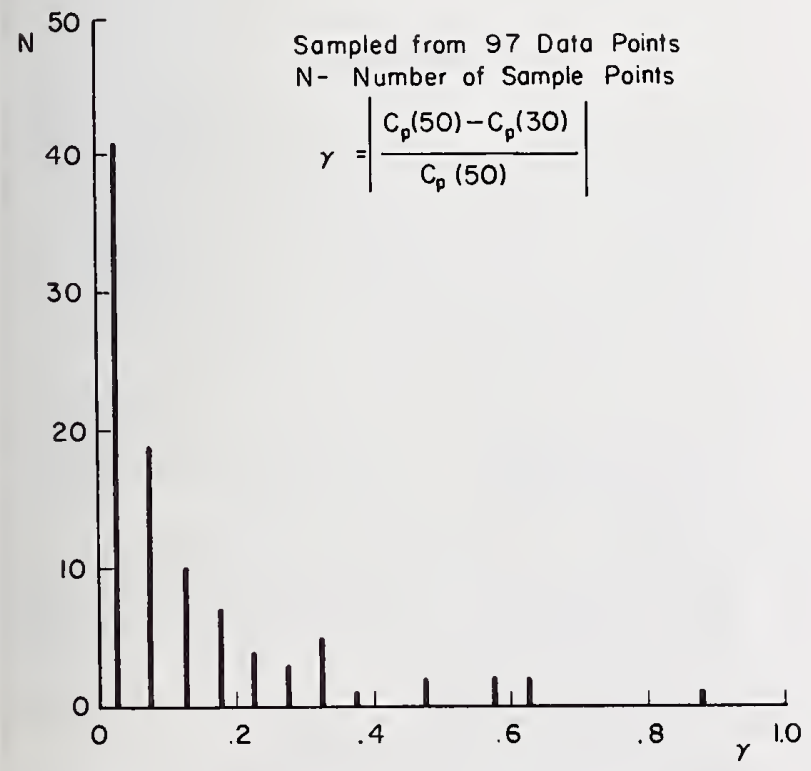

Figure 18. Histogram of normalized pressure coefficient difference. pressure do not yield sufficient information for appropriate estimation of the fluctuating overturning moment. The reason for this is that the correlation of pressure fluctuations on the structure is not known.

The total overturning moment is defined by

$$
M=\bar{M}+m^{\prime},
$$

where $\bar{M}$ is the mean (time-averaged) moment and $m^{\prime}$ is the fluctuating moment. It was measured with a relatively high-frequency response dynamometer system sensitive only ${ }^{+}$) this moment (see Sec. 3.4). Its natural frequency vas about $200 \mathrm{~Hz}$. Based on the pressure fluctuating-energy spectrum most of the energy was concentrated at low frequencies, i.e., up to about $30 \mathrm{~Hz}$.

The measurements were carried out under similar conditions as for the pressure survey, i.e., NE wind of $50 \mathrm{ft} / \mathrm{sec}$. Initially, the moment for smooth upstream conditions, i.e., without the upstream roughness, was measured. Its variation is shown in Figure 20 . The mean moment was about $37 \mathrm{lb}$-in and the peak value of the fluctuating moment reached a maximum of $8 \%$ of the former. All the measurements were reproducible within less than $4 \%$.

Next, a thorough survey of the overturning moment for the real flow conditions, i.e., the upstream roughness installed, was performed. An oscillogram of the fluctuating moment is shown in Figure 19. It reveals a predominant frequency of about $25 \mathrm{~Hz}$. On the other hand, the predominant frequency monitored during the pressure measurement ranged roughly from 15 to $30 \mathrm{~Hz}$ depending on the position along the model. Thus, both measurements indicated the existence of definite predominant frequencies, i.e., a predominant eddy size.

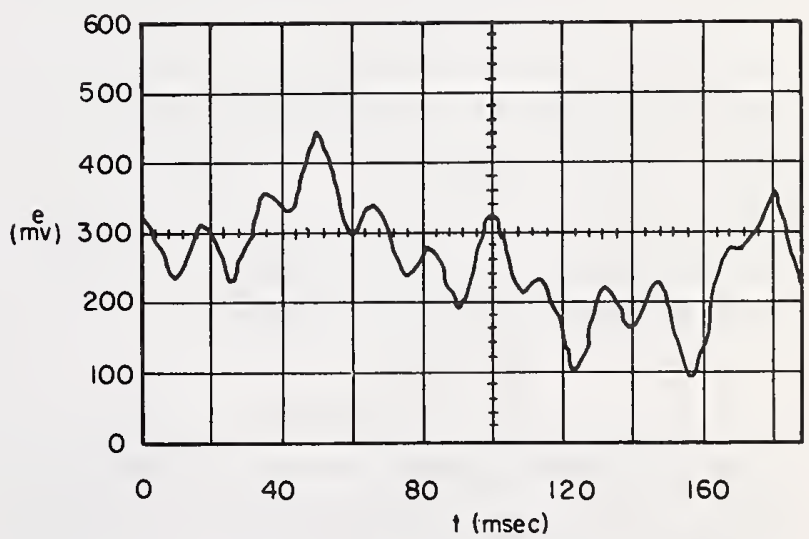

Figure 19. Oscillogram of the fluctuating moment; sweep $20 \mathrm{msec} / \mathrm{cm}$, sensitivity $100 \mathrm{mv} / \mathrm{cm}$. 


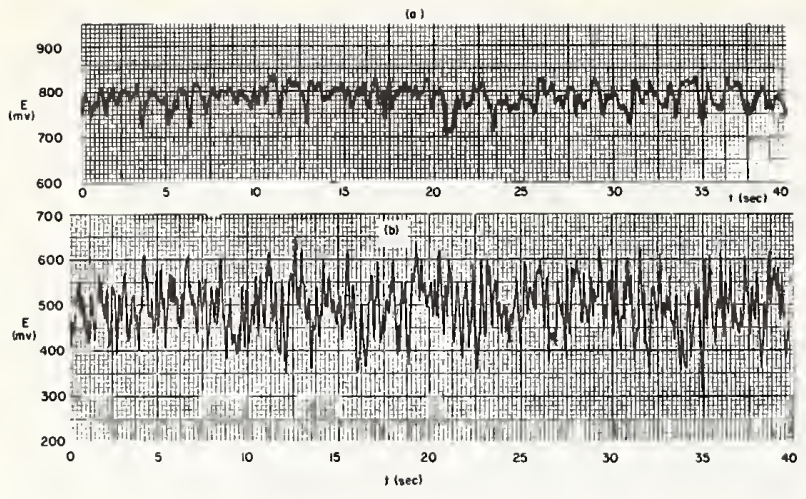

FIgURE 20. Total overturn ing moment variation: (a) without upstream roughness; (b) with upstream roughness.

In Figure 20 the total overturning moment time variation for both cases, i.e., with and without the upstream roughness, is portrayed. The mean moment for the former was found to be about 23.6 lb-in., $37 \%$ smaller than without the upstream roughness. It is smaller since the wind load does depend on the upstream mean velocity distribution.

The instantaneous peak values of the fluctuating moment were found to be of the order of $\pm 34 \%$ of the mean moment. Thus, it was about $8 \mathrm{lb}$-in. This large increase with respect to the smooth upstream conditions is produced by the turbulence caused by the upstream roughness. The rms-value of the fluctuating moment was found to be about $4.8 \mathrm{Ib}-\mathrm{in}$., about $20 \%$ of the mean moment. It is important to notice that the turbulence intensity averaged over the length of the building was roughly of the same order of magnitude.

Lastly, we note that the important result remains that the aerodynamic balance yields immediately a direct and rapid measurement of the total overturning moment. Furthermore, the overall fluctuating moment can be measured accurately and directly. Since the results are obtained for a stationary model structure they may be used as a standard reference loading in numerical analysis for dynamic response of a building with specified elastic and mass distribution characteristics.

\section{Conclusions}

The experimental results presented indicate quite clearly that the direct measurement of the total overturning moment is feasible and desirable. In order to obtain the moment due to the wind action a relatively stiff model has to be used. Both the mean and the fluctuating moment depend strongly on the upstream conditions. Particularly, a strong dependence of the fluctuating moment on the upstream turbulence intensity was observed. A systematic study of this correlation is intended.

Use of the balance technique will permit accurate and rapid measurement of the mean and fluctuating moments. Moments, mean and fluctuating, obtained for a stationary model provide integrated wind loading data which can be utilized in conducting numerical studies of a building with a variety of elastic and mass distribution characteristics. Thus, a standard reference loading is obtained. Furthermore, the use of an aerodynamic balance for measuring other wind loading components is also practicable.

The flow was found to be Reynolds-number independent. No significant changes in the pressure distribution were observed for ambient wind speeds of 30 and $50 \mathrm{ft} / \mathrm{sec}$. The surveys of the mean velocity, turbulence intensity and boundary-layer structure showed that the wind-tunnel flow provided an acceptable simulation of the atmospheric surfacelayer flow conditions.

The peak pressure fluctuation ranged up to about 4 times the mean pressure. Knowledge of the instantaneous local pressure fluctuation is essential for adequate "skin" design of buildings.

The partial support of this work by Metronics Associates, Inc. is gratefully acknowledged.

\section{References}

[1] Cermak, J. E. et al., "Simulation of atmospheric motion by wind tunnel flow," TR CER66JEC-SI17, FDDL, Colorado State Univ., Fort Collins, Colorado (1966).

[2] Davenport, A. G. and Isyumov, N., "The application of the boundary layer wind tunnel to the prediction of wind loading," Proceedings of the Intern. Res. Seminar on Wind Effects on Buildings and Structures, Vol. 1, N.R.C., Ottawa, Canada (1967).

[3] Marshall, R. D. and Cermak, J. E., "Wind studies of Bank of America World Headquarters Building," TR CER66-67RDM-JEC19, FDDL, Colorado State Univ., Fort Collins, Colorado (1966).

[4] Ostrowski, J. S., Marshall, R. D. and Cermak, J. E., "Vortex formation and pressure fluctuations on buildings," Proceedings of the Intern. Res. Seminar on Wind Effects on Buildings and Structures, Vol. 1, N.R.C., Ottawa, Canada (1967).

[5] Jensen, M., "The model-law for phenomena in natural wind," Ingenioren, Int. Ed. 2 (1958).

[6] Jensen, M. and Franck, N., "Model-scale tests in turbulent wind," Part I and II, The Danish Tech. Press, Copenhagen (1965). 
[7] McVehil, G. E., Ludwig, G. R. and Sundaram, T. R., "On the feasibility of modeling small scale atmospheric motions," TR ZB-2328-P-1, Cornell Acro. Lab, Buffalo, N.Y. (1967).

[8] Leutheusser, H. J. and Baines, W. D., "Similitude problems in building aerodynamies," Proceedings ASCE, J. Hyd. Div., HY3, 35-49 (1967).

[9] Armitt, J., "The simulation of the atmospheric boundary layer in a wind tunnel," C.E.G.B., Lab. Note No. $\mathrm{RD} / \mathrm{L} / \mathrm{N} 83 / 66$ (1966).

[10] Pankhurst, R. C. and Holder, D. W., Wind Tunnel Technique (Sir Isaac Pitman \& Sons, Ltd., London, 1952).

[11] Landenburg, R. W. et al., (editors) Physical Measurements in Gas Dynamics and Combustion, Article F, 2, Kovasznay, L.S.G., "Hot-wire method," 219-285 (High
Speed Aerodynamies and Jet Propulsion, Vol. 12, Princeton Univ. Press, Princeton, N.J., 1954).

[12] Sadch, W. Z., Sutera, S. P. and Maeder, P. F., "An investigation of vorticity amplification in stagnation flow," TR AF 1754/4, WT50, Brown Univ., Div. of Ing., Providence, R.I. (1968).

[13] Davenport, A. G., "Gust loading factors," Proceedings ASCE, J. Struc. Div., ST3, 11-34, (1967).

[14] Pavelka, B. R., "Wind study, Atlantic-Richfield Plaza, Los Angeles, California," Met. Report, TR 151, Aerosol Lab., Metronics Associated Ine., Palo Alto, Calif., (1968).

[15] Singer, I. A., "A study of the wind profile in the lowest $400 \mathrm{ft}$ of the atmosphere," PR 5, Brookhaven Nat. Lab. (1960). 


\title{
EXPERIENCE WITH WIND PRESSURE MEASUREMENTS ON A FULL-SCALE BUILDING
}

\author{
W. A. Dalgliesh \\ National Research Council of Canada \\ Division of Building Research \\ Ottawa \%, Ontario, Canada
}

\begin{abstract}
Wind pressure measurements made over a 4-year pcriod on a 34-story building in downtown Montreal werc used to obtain data for chceking and improving wind tunnel techniques of modcling flow characteristics of wind and acrodynamic bchavior of buildings. It had been hoped that the measurements could be applied dircetly to certain problems of design such as evaluation of pcak suction load over small wall areas. The small number of pressures recorded, however, combincd with limitations of field measurements made direct application of the data extremcly difficult.

The major problems involved in making field measurcments and in comparing them with wind tunnel measurements were found to be:

(a) difficulty of establishing a static reference pressure and its relation to the static pressure in the wind tunnel;

(b) inadequacy of wind velocity information, which in this case consisted of one anemometer and wind vane located $1,500 \mathrm{ft}$ southwest of the building;

(c) lack of stationarity and homogeneity of the velocity field as compared with the wind tunnel situation.

Comparisons with model measurements are made on the basis of mean pressures, rms pressures, power spectra, and the correlation between selceted pairs of pressures measured at various points on the building. Examples have been found of cxcellent agreement in almost all respects, but for some wind directions the comparisons gave unsatisfactory eorrelation. The lack of agreement is attributed mainly to differences between indicated and actual on-site wind dircetion, but this cannot be shown conclusively because of incomplete wind information.

The total eost of the project over the 4-year period was of the order of $\$ 100,000$. A greater expenditure would have been advisable, primarily for instrumentation to permit a better definition of the wind velocity around the building. Measurements are now under way on a $600-\mathrm{ft}$ office building using a much more sophisticated data acquisition system by mcans of which it should be possible to acquire the desired information more efficiently and in a shorter period of time.
\end{abstract}

Key words: Buildings; full-scale tests; power spectra; pressure fluctuations; wind loads; wind tunnel modeling.

\section{Development of Methods for Wind Research}

Investigation of wind effects on buildings and structures by the Division of Building Research, National Research Council of Canada, began in 1958 when an extensive survey of the available literature [1]* was made in connection with the revision of design wind load information for the 1960 edition of the National Building Code. In this code, as in most other building codes, the conversion from design wind speeds to design wind pressures and suctions on various building surfaces was given in the form of pressure coefficients determined experimentally by testing small-scale models in wind tunnels.

\footnotetext{
* Figures in brackets indicate the literature references at the end of this paper.
}

\subsection{Need for Full-Scale Data on Pressure Coefficients}

Study of the literature revealed serious discrepancies among pressure coefficients for geometrically similar models tested by different researchers. Modeling laws were obviously a matter for debate, which brought into serious question the applicability of the results to full-scale structures [2].

A need clearly existed for field measurement of wind pressures on full-scale buildings to answer the questions of applicability to full-scale situations, and the correctness of similitude rules. There was surprisingly little full-scale information available, however, up to 1960 , and the few comparisons with model results that had been made were at best inconclusive. 


\subsection{Objectives for Field Measurements in Canada}

A project was therefore set up within the Structures Seetion of DBR/NRC to measure pressures and suetions on full-scale buildings. The main objeetives were to eheek wind tunnel data and possibly to indieate how to improve modeling techniques. It had been thought at first that such field information might also prove directly useful to designers in assessing, for example, peak suctions over small areas or for similar problems of detailed eonditions in whieh wind tunnel results seemed inadequate. The limited instrumentation, however, coupled with other problems to be discussed in Section 3, made direet design application of these particular measurements very difficult.

\subsection{Full-Scale Measurements in Other Countries}

In eontrast with the limited aetivity before 1960 , interest in field investigations of wind effects on structures sinee that time has been very great. In England an extensive program of full-seale measurements of wind pressures has been under way for nearly 10 years, and interim results from measurements on a 200 -ft tall slab-like office building have been reported $[3,4,5]$. The program for the future involves measurements on the new 600-ft G.P.O. tower in London.

Full-scale measurements of wind pressure were also earried out on a $150-\mathrm{ft}$ slab-like university building in Melbourne, Australia. Results were eompared with wind tumel tests of a conventional type and with those using a turbulent boundary-layer flow [6]. Dissatisfaetion with the eorrelation achieved led to more fundamental laboratory work on the flow around wall-mounted bluff objeets (buildings on the ground).

Wind loading of a 145 -ft slab-like apartment building in Delft, Holland, was investigated without the use of pressure taps, using instead a single deformation gage mounted on one of the steel columns [7]. These results were correlated to some extent with calculations based on the statistical approach to wind loading proposed by Davenport [8].

The widespread interest in full-seale measurements shows no sign of slaekening. Construetion is now well advaneed on a 10-story welded steel-frame building in Hong Kong to be used exclusively for full-seale research on wind forces during typhoons [9].

\subsection{New Developments in Wind Tunnel Techniques}

The speeial problem of building aerodynamies as opposed to eonventional aeronautieal wind tunnel work has also been receiving attention [10], with great emphasis on simulating the turbulent shear flow usually found during strong winds. One method of ereating a turbulent shear flow that has been used with considerable success involves the socalled boundary-layer wind tumnel, in which a thiek boundary layer is allowed to grow over the long, specially roughened floor of the tunnel. Over the past 3 or 4 years the boundary-layer wind tunnel has been applied as a design tool for predicting wind loading on several major struetures [11].

The parallel development of laboratory and field techniques, and the availability of full-scale results for comparison with model results have already explained some of the discrepaneies of earlier wind tunnel work, but much checking and improvement remains to be done. The measurement of wind pressures and suctions on a full-scale building in downtown Montreal, Canada, eonstitutes the most reeently completed portion of the investigations under way in the Structures Seetion of DBR/NRC. These results have been compared with wind tunnel tests done in a boundary-layer wind tunnel. A brief description of the field measurements, of the problems of analysis and interpretation of the results, and of the correlation with wind tunnel results follows.

\section{Instrumentation of 34-Story Office Building}

Arrangements were made with the owner of a 34story office building in Montreal to permit the instrumentation of the two mechanieal floors for wind pressure measurements. Most of the offiee floors of the building had already been rented at the time of instrumentation (spring, 1964), and this made it impossible to instrument other levels without seriously inconveniencing the tenants. The mounting of an anemometer and tower on the building itself was not praeticable, and wind speed and direetion signals were transmitted through telephone wires from instruments mounted in the fall of 1964 on an existing $200-\mathrm{ft}$ mast atop a $600-\mathrm{ft}$ building to the southwest.

\subsection{Influence of Surrounding Terrain}

The location of the two buildings involved and the nature of the surrounding terrain are shown in Figure 1. As may be seen from the ground contours, 


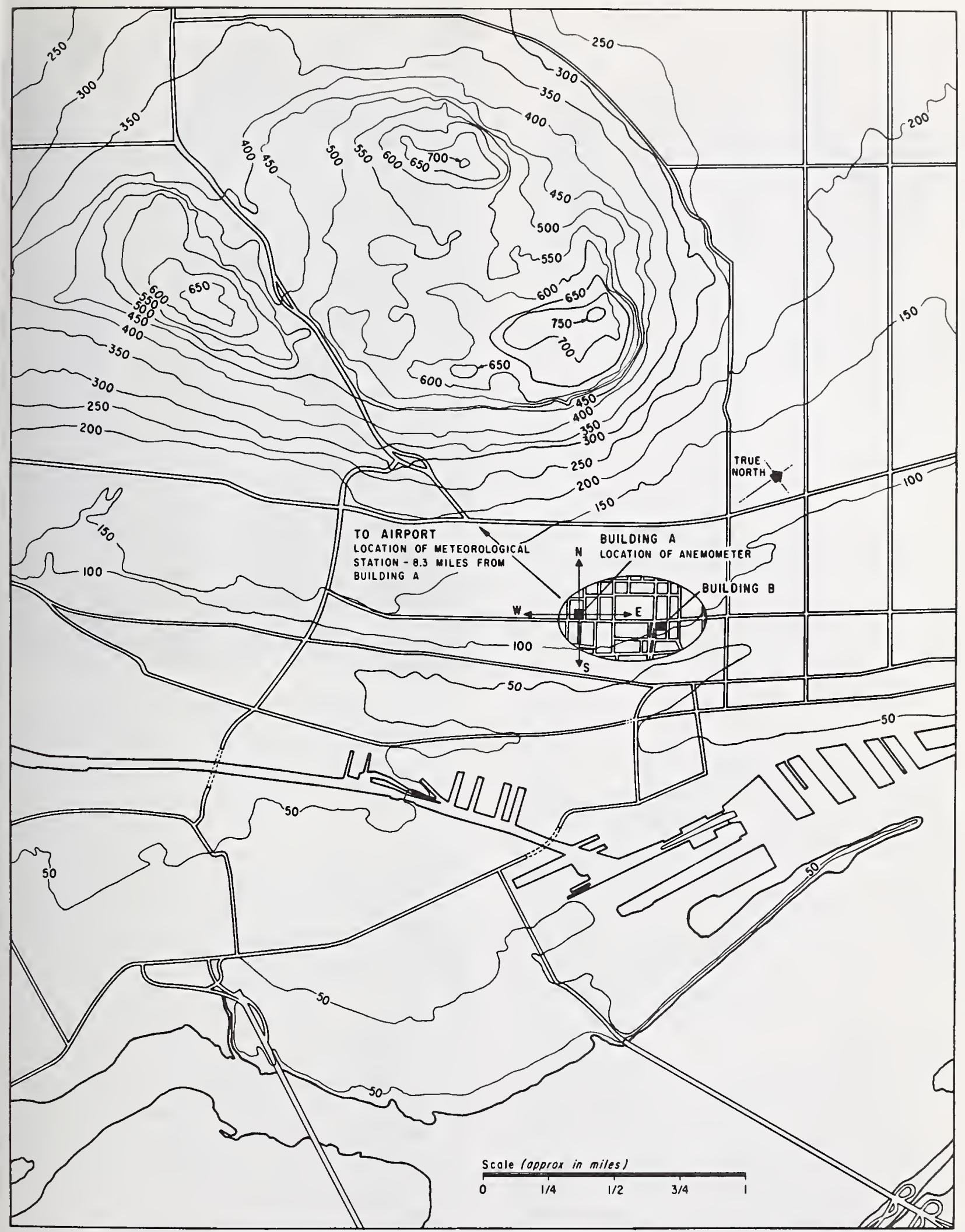

Figure 1. Contour map of the terrain surrounding test buildings. 


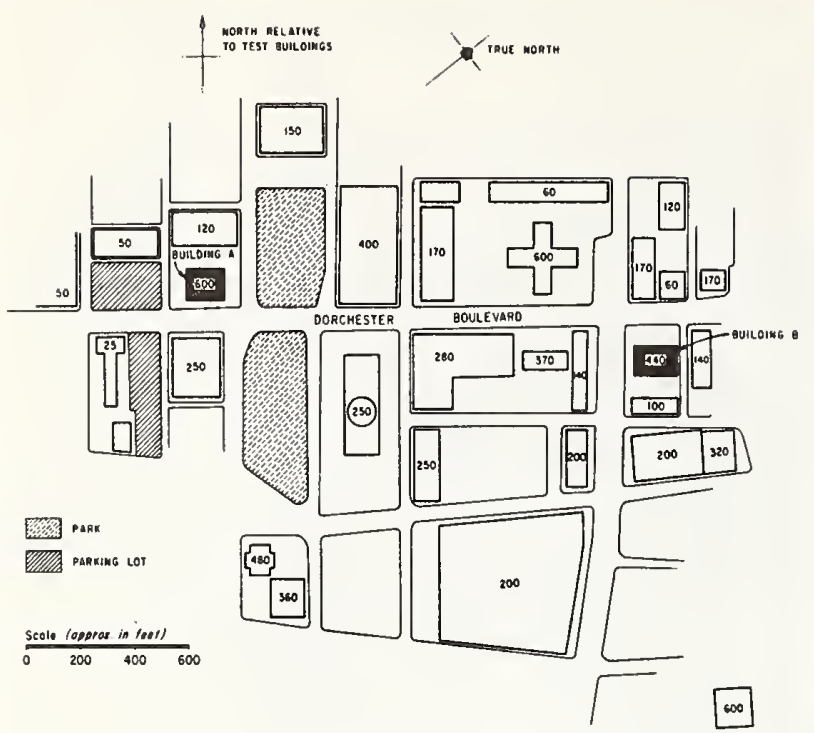

FIgURe 2. Plan showing heights of adjacent buildings.

the area is fairly level except for Mount Royal to the northwest. Figure 2 is an enlarged view of the immediate surroundings. Wind pressures on the 34story building (building B) were found to be significantly influenced by the presence of the nearby tall buildings, particularly the $600-\mathrm{ft}$ cruciform building to the northwest.

\subsection{Installation of Pressure Taps}

The instrumented floors are the 10th and the $33 \mathrm{~d}$, at heights, respectively, of 134 and $413 \mathrm{ft}$ above the street. The total height of the building is $440 \mathrm{ft}$ and the plan dimensions are 119 by $173 \mathrm{ft}$. The exterior wall surface is plane except for 8-in. deep mullions, $4 \mathrm{ft} 8 \mathrm{in}$. apart, which run the full height of the building. At the mechanical floors, however, there are horizontal louvers, and the curtain wall is set back about $5 \mathrm{in}$. from the plane of the exterior wall. The louvers show up as dark bands on the wall surfaces in figure 3 .

Holes $1 / 8$-in in diameter were drilled through the curtain wall into the space behind the louvers about $2 \mathrm{ft}$ above floor level at intervals of approximately $24 \mathrm{ft}$ all around the building at the two levels, except where locations were inaccessible because of equipment placed in the way. Any 12 of a total of 49 holes could be used as outside pressure taps for the 12 transducers, which were used to convert pressure differences into electrical signals for convenient transmission to a central recording location.

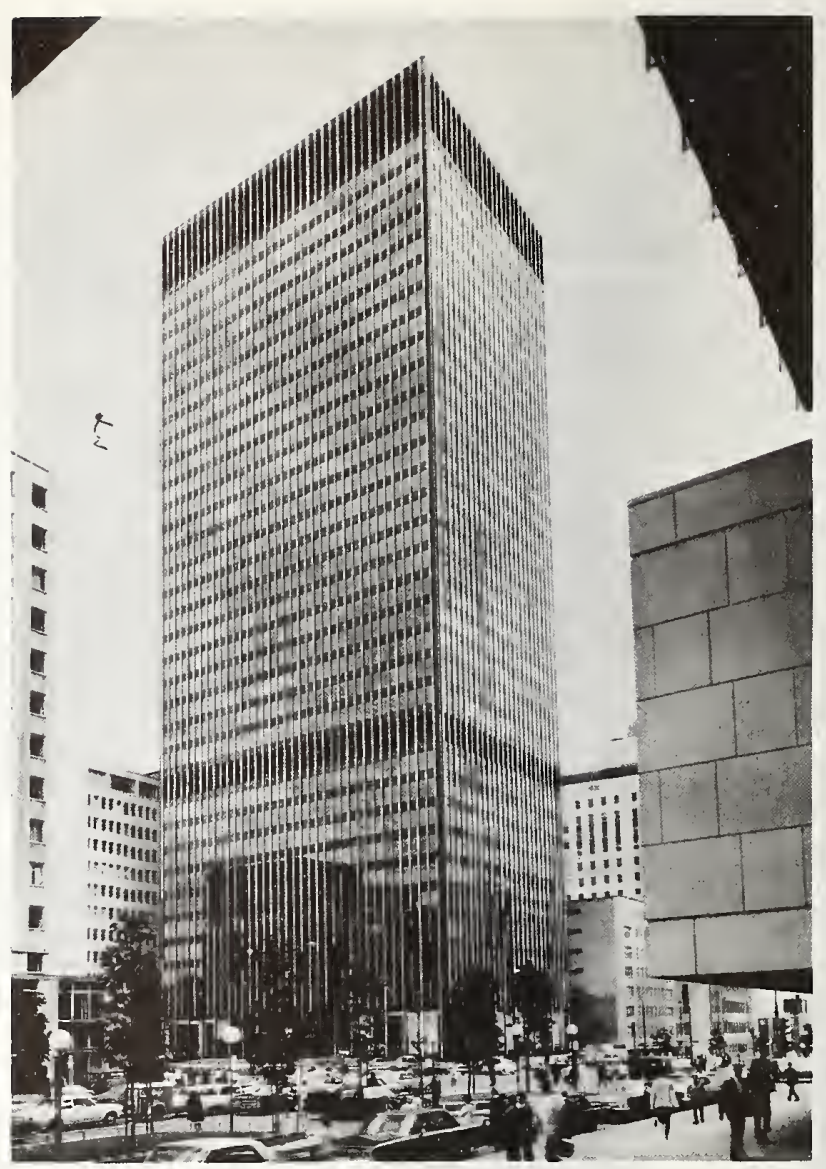

Figure 3. Thirty-four-story office building looking southeast (relative to building). Dark bands are at 10th and $33 \mathrm{~d}$ floors, where mechanical services are located and wind pressure measurements were taken.

\subsection{Sensors and Recording Equipment}

The turbulent nature of wind requires that speed, direction, and pressure at several locations on the building be recorded simultaneously and continuously for periods preferably longer than 10 or 15 minutes at a time. The response of the sensors and the capacity of the recording equipment should be sufficiently high to record fluctuations with frequencies up to several cycles per second. The equipment used for the instrumentation of the 34-story office building met most of the requirements, but in the final analysis of the records an upper frequency limit of 0.5 cycle/sec was imposed to keep the task of manually preparing digitized records within reasonable bounds.

The pressure sensor, or transducer, of which there were 12 , comprises an elastic steel diaphragm dividing a chamber connected, on one side, by about $2 \mathrm{ft}$ 
of plastic tubing to the outside air pressure tap, and on the other side by up to $200 \mathrm{ft}$ of plastic tubing to the air pressure at a central location inside the building. The pressure difference between the two sides of the chamber is measured in terms of the diaphragm deflection by an unbonded 4-arm resistance strain gage bridge. The power and signal are transmitted by strain gage cables over distances of up to $600 \mathrm{ft}$ to the central recording location on the 10th floor.

The wind vane and 3-cup anemometer are of the type U-2A used by the Meteorological Branch of the Canadian Department of Transport and were located about 1,500 ft away at a height of $800 \mathrm{ft}$ above street level on building A (see Figs. 1, 2). Electrical wind speed and direction signals, transmitted via telephone lines, were recorded with the 12 pressure signals on an 18-channel ultraviolet light beam type oscillograph. Chart speed was limited to $4 \mathrm{in} . / \mathrm{min}$ for most of the recording, and the full-scale deflection of each signal was made \pm 2 in. ( \pm 10 psf).

\subsection{Field Trips and Processing of Records}

Trips were made from Ottawa to Montreal (120 miles) whenever strong winds seemed imminent. It was necessary upon arrival to select the 12 most desirable tapping locations (depending on the wind direction), set up transducers, and begin recording. Set-up time was usually about 1 hour and recording usually continued for 1 to 3 hours longer. Of the results obtained on eight such trips between 5 March 1964 and 23 September 1966 five sets were selected for detailed study.

After visual examination of each strip chart record, portions represcnting recording intervals from $15 \mathrm{~min}$ to over an hour were selected and digitized, using a semiautomated procedure, at time intervals of 1 sec. The digitized values, either on punched cards or digital magnetic tape, were then processed by a digital computer.

\section{Differences Between Field Measurements and Wind Tunnel Measurements}

The model scale investigations were madc in a boundary-layer wind tunnel rather than in a conventional low-turbulence aeronautical wind tunnel because of the undoubted importance of modeling the gustiness of real wind. Care was taken to simulate field conditions as closely as possible, but in spite of this certain differences remain. As an example of the precautions taken, the model of build- ing $B$ was carefully machined from plastic to a scalc of 1:400, including the mullions. Pressurc taps were made at locations corresponding to those where full-scale taps on the two instrumented levels were situated, and three additional levels were tapped to give a more complete picture of pressure distribution than was possible in the field measurcments. All major structures within a $1,600-\mathrm{ft}$ radius were modeled to the 1:400 scale from wood, and upwind land contours and surface roughness were also simulated, as is shown in Figure 4.

A fundamental difference between the field measurements and the wind tunnel measurements is related to control of flow conditions and the reproducibility of an experiment. The main advantage of wind tunnel testing over full-scale testing is the fact that investigations can procced systematically and efficiently. Full-scalc measurements, on the other hand, are dependent on the random behavior of weather, making systematic investigations impractical. This is particularly true for such features as the phenomenon of extreme suctions near the corners of the buildings, for which wind angle is fairly critical.

\subsection{Stationarity and Homogeneity of Flow}

The basic difficulty associated with field measurements arises bccause of the random nature of wind. Not only is the acquisition of useful records made difficult and time-consuming, but the interpretation and comparison with laboratory results may become confusing. Much of the confusion can be avoided if a distinction is made between "weather" and "gustiness" on the basis of the time scales involved. The shortest period associated with weather changes and, in particular, strong winds is usually an hour or more, except for thunderstorms, whereas the longest period associated with gustiness is about 5 minutes. Thunderstorms may have to be treated somewhat differently from other types of strong wind storms.

The randomness of the gusts superimposed on the mean wind speed can be analyzed using statistical procedures developed over the past 20 year's by communications engineers and others [12, 13]. These statistical procedures have been applied recently to wind effects on structures [8]. The application of established methods for measurement and analysis of random data is greatly simplified if it can be assumed that the wind is at least weakly stationary and homogeneous. This implies that the means and variances of wind velocity and pressures are con- 


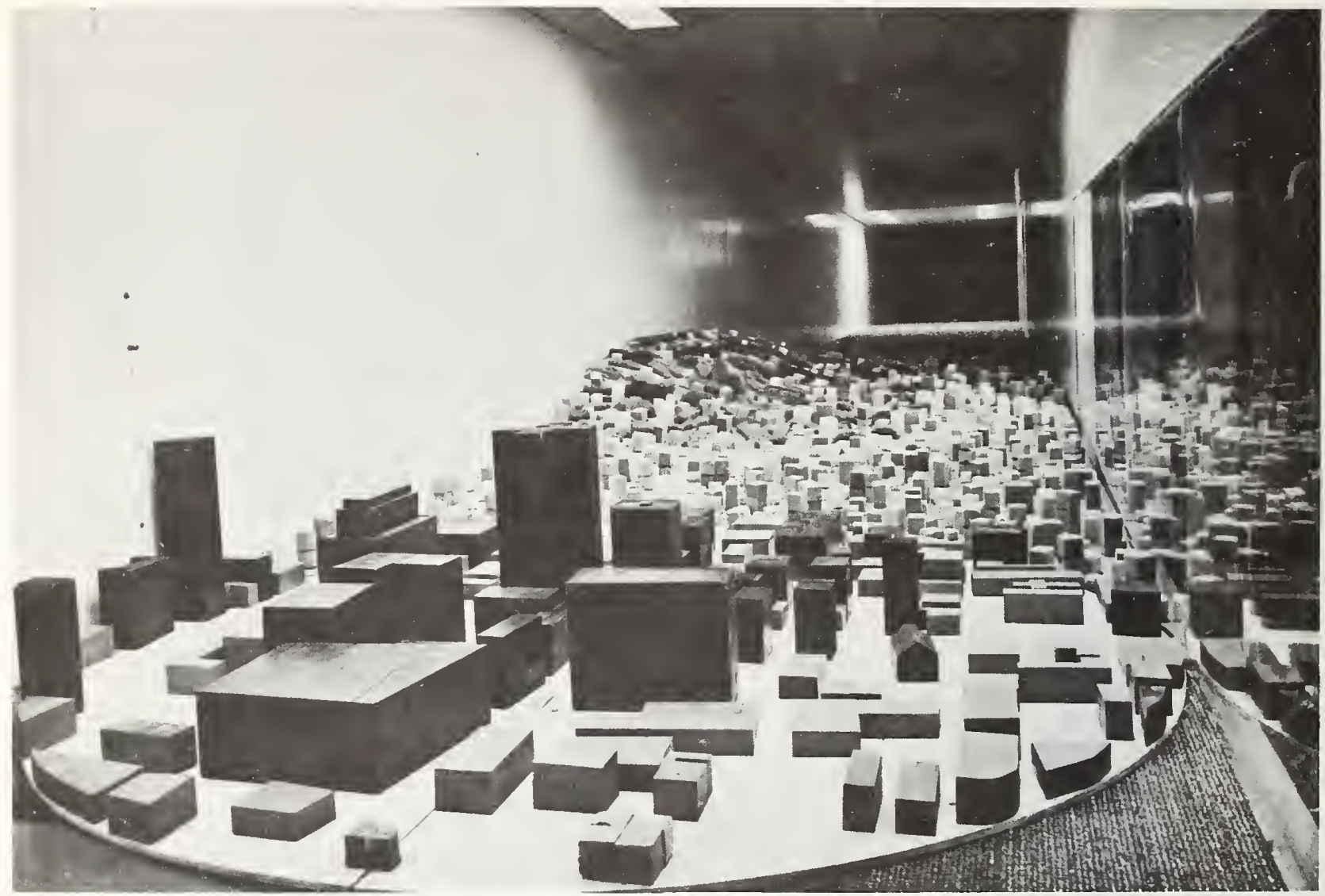

Figure 4. Model of downtown Montreal with major topographical features in the boundary-layer wind tunnel at the University of Western Ontario for comparison with full-scale measurements.

stant with respect to time and with respect to position in space. Stationarity and homogeneity hold only approximately for field measurements, if at all, and then only if care is used in selecting suitable portions of the total record for detailed analysis. In principle, it would be best to use as long a record as possible to improve the reliability of estimates of means, variances, and distributions of variances according to frequency (power spectral density). It was found, however, that portions of record much over 5 to 10 minutes often contained "trends" or variations in the mean value, and these complicated the analysis.

There was no particular difficulty in satisfying the requirements of stationarity in the wind tunnel, but in consideration of the objective of simulating field conditions it was thought more important to reflect the nonhomogeneities of the prototype situation rather than to have the theoretical advantages of the homogeneous flow. The land contours of the eastern end of Mount Royal were, therefore, modeled in order to introduce the same sort of spatial variation of velocity as might be found in the field (Fig. 4).

\subsection{Time Scaling}

The time interval over which mean velocities and pressures were averaged was chosen to distinguish between (a) the random fluctuations about the mean, and (b) the much slower variation of the mean wind velocity and the corresponding pressures in response to changing weather conditions. A scaling parameter with respect to time must therefore be considered when comparing field and model measurements.

For purposes of comparing averaging times the nondimensional parameter to be kept the same for model and full scale is:

$$
\frac{V t}{L}
$$

where $V=$ mean velocity in $\mathrm{ft} / \mathrm{sec}$,

$t=$ time in sec,

$L=$ characteristic length in $\mathrm{ft}$.

The ratio of mean velocity in the tunnel to mean velocity in the field ranged from about 0.50 to 0.75 , and the length scale for the model was 1:400. An averaging time of $30 \mathrm{~min}$ in field measurements 
would therefore be represented by a tunnel time of 6 to 9 sec. The averaging time actually used in the tunnel was $30 \mathrm{sec}$, and the length scale of the gusts proved to be approximately 1:700; the corresponding full-seale averaging time would be about 175 to $260 \mathrm{~min}$, based on the scale of turbulence. A much longer averaging period can be used in the wind tunnel because the slow-moving trends assoeiated with the weather system in the field situation are not present.

\subsection{Reference Static Pressure}

Wind tunnel measurements of pressures on a tapped model are expressed in terms of differenees from a reference static pressure, divided by a reference dynamic pressure. The resulting nondimensional ratio, the pressure eoeffieient, is defined as follows:

$$
C_{p_{i}}=\frac{P_{i}-P_{o}}{1 / 2 \rho V^{2}}
$$

where $C_{p_{i}}=$ pressure coefficient,

$P_{i}=$ pressure at $i$ th tap,

$P_{o}=$ referenee statie pressure, $\mathrm{lb} / \mathrm{ft}^{2}$,

$\rho=$ mass density of air, slugs $/ \mathrm{ft}^{3}$,

$V=$ reference mean velocity, $\mathrm{ft} / \mathrm{sec}$.

The usual referenee static pressure $P_{o}$ is the annbient barometric pressure inside the tunnel, measured either at a flush wall tap or at the statie side of a pitot tube mounted upwind of the model.

The reference side of each transdueer in the field measurements was connected by a long plastic tube to the ambient barometric pressure at a point near the eenter of the 9 th floor of the 34-story building. The reference tubing was present to ensure that all transdueers were at least measuring with respeet to a eommon referenee pressure, even though it was not neeessarily eompletely statie.

The difference between the referenee statie pressure in the field and that used in the tunnel proved to be one of the most troublesome aspects of the comparison of results. The difference, of the same order of magnitude as the measurements themselves, was caused by a combination of factors:

(a) ehimney aetion-the temperature differential during cold months, eombined with the very considerable effeetive stack height, caused pressure differences of the order of 2 to 5 $\mathrm{lb} / \mathrm{ft}^{2}$;

(b) operation of the air-handling equipmentbuilding pressurization of as mueh as 1 or 2 $\mathrm{lb} / \mathrm{ft}^{2}$ was common;

(e) wind effect-assuming an internal pressure coefficient of -0.3 , the action of the wind could produce a lowering of the internal pressure by 1 or $2 \mathrm{lb} / \mathrm{ft}^{2}$.

Simulation of these effects would be extremely complicated in the wind tunnel because of the very eomplex system of flow resistances and leakages throughout the building. As a result, no adjustment was made to the wind tunnel technique; a eorreetion was made, instead, in the comparison of the pressure eoefficients.

\subsection{Reference Dynamic Pressure}

The dynamic pressure of the reference mean velocity of equation (1) was measured in the wind tunnel at a height of 24 in. midway between building A and building B (see Figs. 1 and 2). This height corresponds to $800 \mathrm{ft}$ in actual field measurement, but the pitot tube had to be moved away from building A to avoid the interferenee effect of the tunnel wall.

The use of this dynamie pressure as a reference in the wind tunnel should not seriously affeet eomparison, provided the spatial variation of velocity is small from building $\mathrm{A}$ to the pitot tube loeation, and similar in model and full scale.

\section{Comparison of Ficld and Wind Tunnel Results}

When the mean pressure eoeffieients, $C_{p_{i}}$, derived from the field measurements, were first eompared with the $C_{p_{i}}$ from the wind tunnel, allowance had not yet been made for the difference in reference statie pressure. The comparison was consequently quite unsatisfactory. The effects of chimney action and building pressurization by the air-handling system were frequently suffieient to overeome the wind pressure on the windward wall. The transducers at all locations around the building therefore registered suctions in relation to the referenee internal pressure.

A eorreetion was then applied to the reference static pressure at eaeh level for each of the reeords of full-scale measurements. Corrections were calculated using the least squares prineiple to produce the best fit of the full-seale pressure distribution to the model pressure distribution. This procedure seems reasonable as long as there is a definite eorrelation between the two sets of pressure coefficients and the deviations that remain after fitting appear to be small and random. It is interesting to note that cor- 
rections made in this way to the reference static pressure are independent of the dynamic reference pressure. The same correction of the reference static pressure (in $\mathrm{lb} / \mathrm{ft}^{2}$ ) is obtained even if completely different dynamic reference pressures are used in the wind tunnel and in full scale. The corrections arrived at by least squares fitting were checked in each instance and found to be consistent with approximate calculations of chimney action and building pressurization.

\subsection{Comparison of Mean Pressure Distribution on 34-Story Building}

The relation between full-scale and model mean pressure distributions in terms of the nondimensional pressure coefficients, $C_{p_{i}}$, is plotted for two different wind directions in Figures 5 and 6 . The agreement in the first case (wind at right angle to building), particularly at the $33 \mathrm{~d}$ floor level, is very good with regard to both distribution and scale. Many similar portions of record for approximately the same indicated wind angle gave equally good

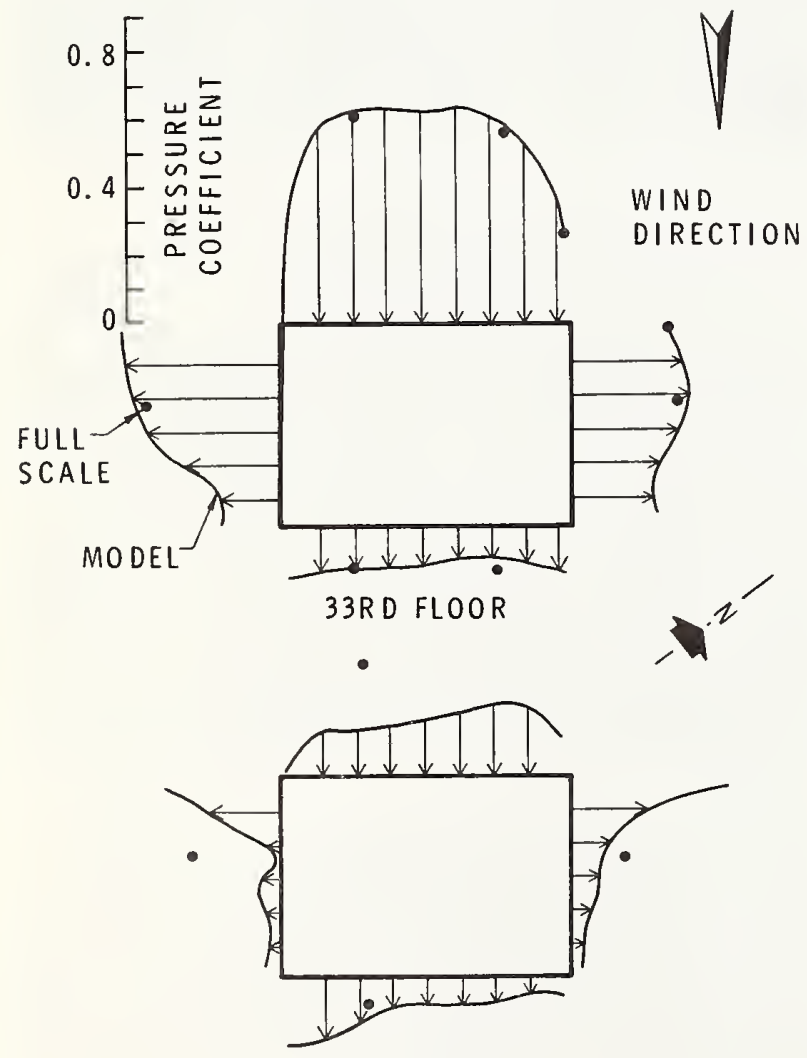

10TH FLOOR

Figure 5. Comparison of full scale with model results; mean pressure coefficients on 34-story office building, northwest wind. agreement in terms of pressure distribution, but no other sample was as close to the model results in terms of dynamic pressure, which governs the scale of the pressure distribution.

Figure 6 does not show the same agreement for distribution. The probable explanation for suction on the southwest wall of the $33 \mathrm{~d}$ floor in the fullscale result is that the indicated wind direction at building $\mathrm{A}$ (where the anemometer and vane are located) may have differed by perhaps as much as $10^{\circ}$ from the actual wind direction at the building. If such a difference existed it was evidently not simulated by the model.

\subsection{Comparison of Mean Pressure Distribution in the Empire State Building}

Agreement between wind tunnel tests and fullscale tests, unless it is either uniformly good or com-
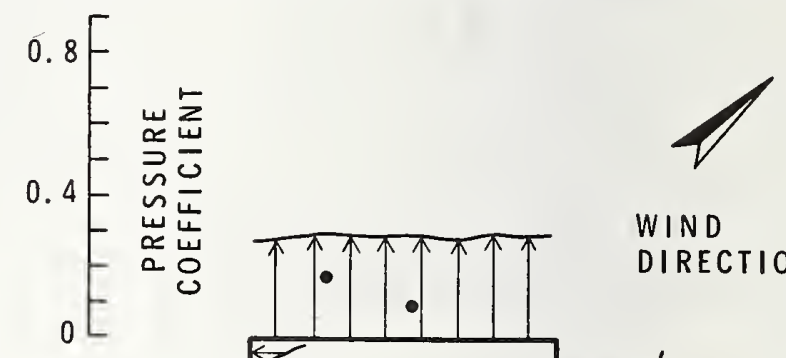

WIND

DIRECTION
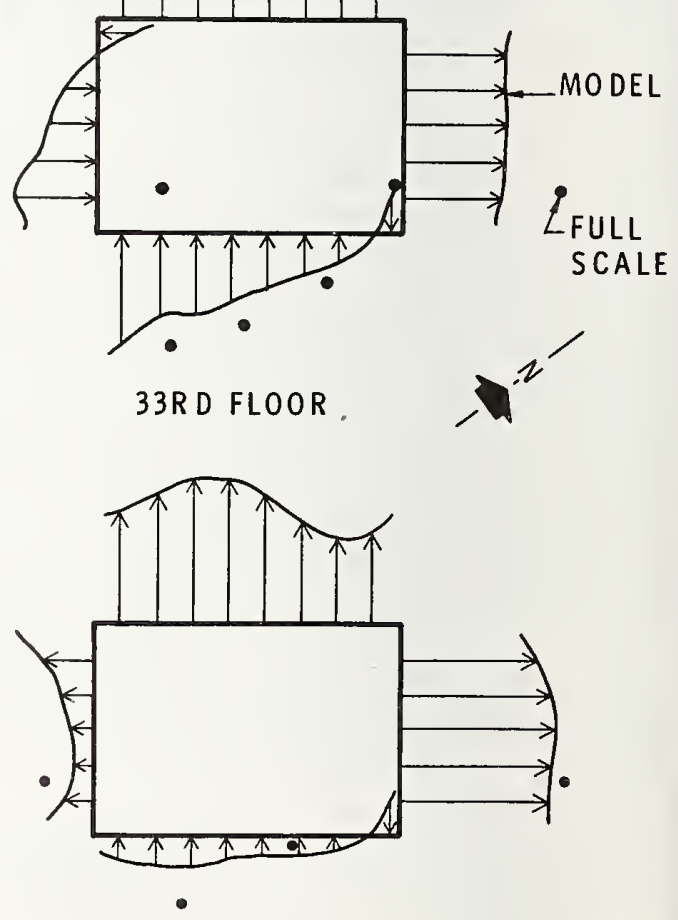

10TH FLOOR

Figure 6. Comparison of full scale with model results; mean pressure coefficients on 34-story office building, south wind. 


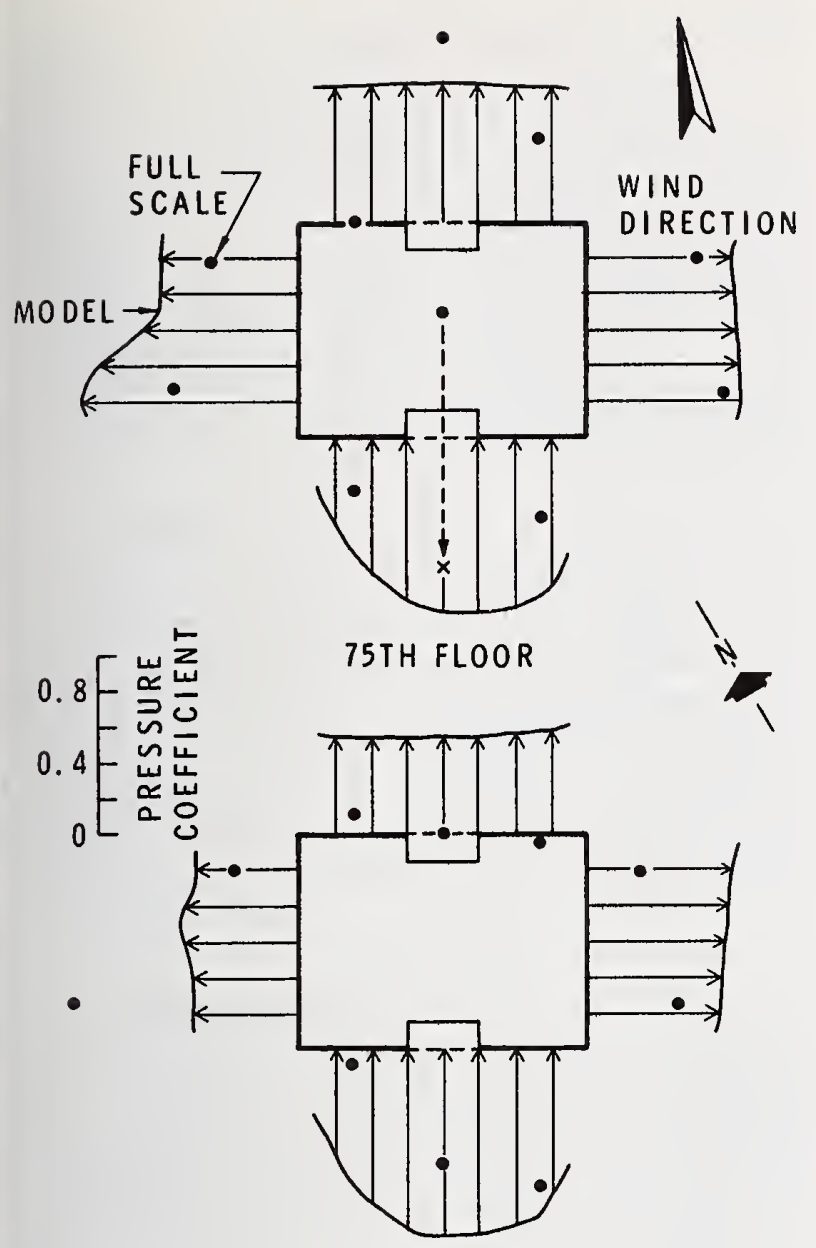

36TH FLOOR

FIgure 7. Comparison of full scale with model results; mean pressure coefficients on Empire State Building, SSW wind (from Rathbun, Dryden, and Hill).

pletely unsatisfactory, is hard to assess in objective terms. To help establish some perspective for making at least a subjective assessment of the results the author studied comparisons made by others who had done full-scale tests $[5,6]$.

Of particular interest were measurements on the Empire State Building, reported nearly 30 years ago by Rathbun [14]. The wind tunnel tests for this building were carried out at the National Bureau of Standards and the results were presented in 1933 by Dryden and Hill [15]. No comparison of the pressures on the model and those on the building was presented in the paper by Rathbun, presumably because there appeared to be very little agreement.

The author therefore made several comparisons similar to the two illustrated in Figures 5 and 6 for the 34-story building. Agreement was poor for
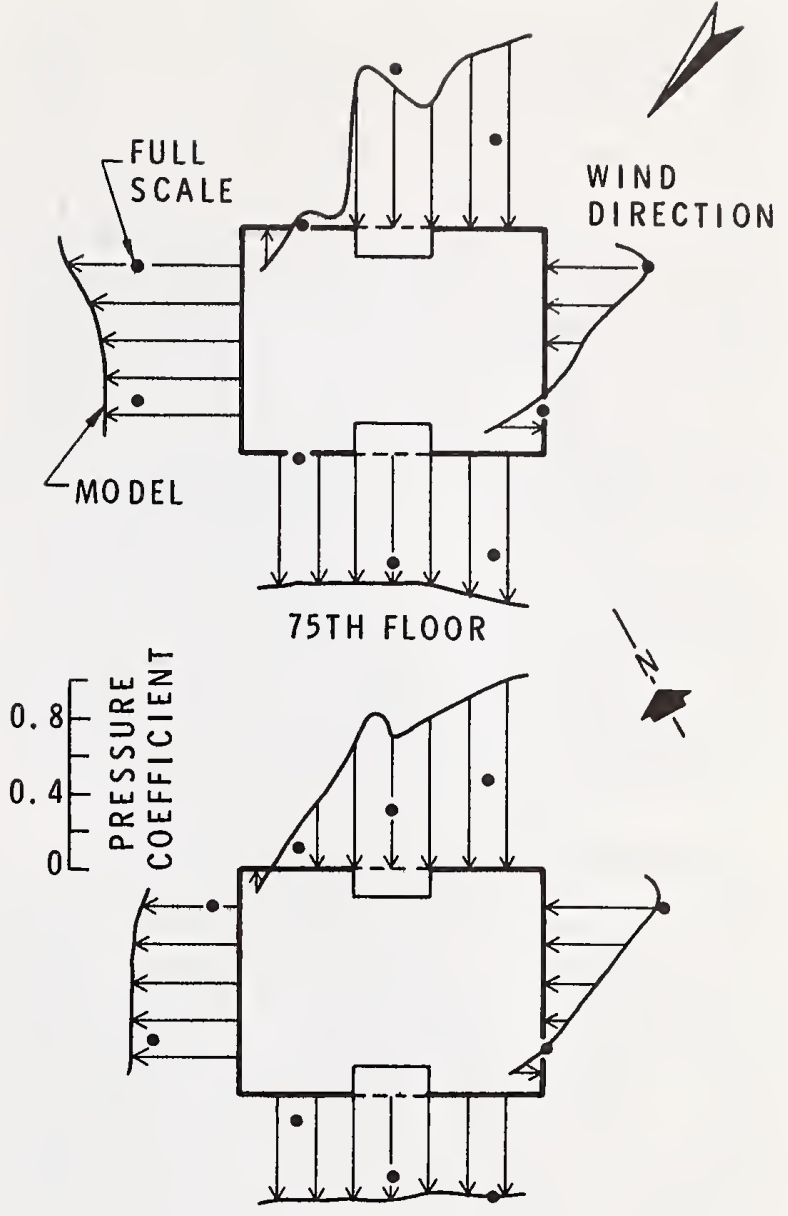

36TH FLOOR

Figure 8. Comparison of full scale with model results; mean pressure coefficients on Empire State Building, ENE wind (from Rathbun, Dryden, and Hill).

some of the "items" tabulated by Rathbun. There were, however, other items for which the agreement in pressure distribution was good, except that the scale (i.e. the reference dynamic pressure) seemed considerably smaller for the full-scale results. The two examples given in Figures 7 and 8 were chosen to parallel as closely as possible the examples of Figures 5 and 6 . One reading on the 75th floor in Figure 7 seems to be a clear case of mistaken sign, and has been plotted at its probable value as a cross.

The deviations from the model pressure distributions in Figures 7 and 8 are rather larger than those in Figures 5 and 6 , and the scale is consistently smaller. The improved agreement of the recent comparison can be attributed to improvements in both the instrumentation of the full-scale building and the modeling techniques used in the wind tunnel. 
The disparity in scale of the work on the Empire State Building probably results from use of a constant velocity profile and the absence of terrain roughness or the shelter of other buildings in the wind tunnel. A similar difference in scale would no doubt have been found for the Montreal building if the effects of terrain roughness and the other tall buildings nearby had not been simulated. Thirty years ago pressures were measured by $\mathbf{U}$-tube manometers, either by observers or by photographing the manometer banks; in either case it was difficult to get an accurate record of mean pressures. Much of the scatter and disagreement of the results must have been caused by gustiness in the wind that could not be accounted for in the analysis of the records. Modern data acquisition systems now make it possible to distinguish between mean and gust readings to a large extent and to treat mean and fluctuating components separately.

\subsection{Analysis of Fluctuating Components}

For purposes of analysis the fluctuating component of a record of wind speed or pressure versus time is treated as a stationary random process. The parameters estimated by analysis of the records are useful for determining equivalent static loads or for estimating the probable number of load cycles at different stress levels. It is consequently a matter of considerable importance to demonstrate agreement between full-scale and model results for parameters relating to the fluctuating component such as standard deviation, or rms pressure coefficients, and power spectral density as a function of frequency.

Agreement of shape and location on the frequency axis of the power spectral density curve implies a proper scaling of the turbulence in the wind tunnel. Figure 9 is a combined plot of both full-scale and

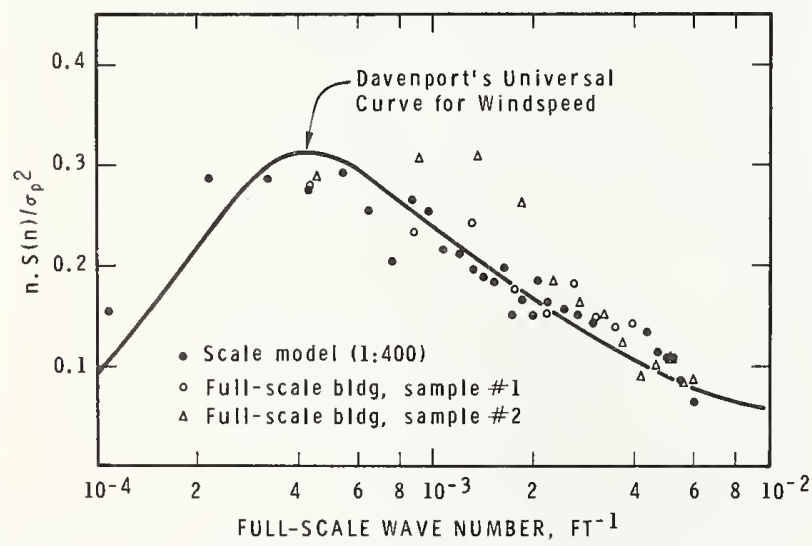

Figure 9. Sample power spectral density curves obtained from both model and full-scale experiments on an office building in Montreal. model information for the pressure records obtained at a corresponding tap location on the $33 \mathrm{~d}$ floor on the windward wall. An empirical curve based on wind speed data, suggested by Davenport, is also shown. The scale of turbulence was probably about 1:700 in this particular model test, and did not therefore agree with the length scale of the building $(1: 400)$. The ratio of velocities happened to compensate for the difference to a large extent, and as a result the spectral peaks appear to occur at approximately the same reduced frequency.

\section{Evaluation of Field Measurements}

The sample comparisons in Section 4 are fairly representative of the sort of agreement that has been found between wind tunnel tests in a boundarylayer type wind tunnel and measurements of wind pressure on the full-scale building in Montreal. The need for considerably more full-scale information is indicated although the results can be considered encouraging in many respects.

\subsection{Suggested Improvements}

Several improvements in instrumentation and data processing can be suggested on the basis of experience with that part of the project now completed. There is merit in recording data in a visual form, such as an oscillograph chart, for pilot studies or short-term projects. For the main part of an investigation, however, data processing should be handled by computer. The data acquisition should therefore employ punched cards, paper tape or magnetic tape storage, compatible with computer input requirements.

A second improvement would be an automated system for initiating recording whenever suitably strong winds occur. Approximately five of eight trips to Montreal produced successful runs in the project just completed but many good storms were undoubtedly missed because of insufficient forewarning.

The third suggestion for improved procedure concerns the scheduling of wind tunnel tests. One of the weaker areas of field installations is often the acquisition of wind velocity records. On the one hand, one can strive for more and better anemometer sites; on the other, maximum use should be made of the wind tunnel in searching for those conditions, particularly angle of attack, that best simulate a particular field experiment.

Measurements of building accelerations, strains in columns, or deflections can provide valuable information about the integrated effect of wind over 
the whole structure, and comparisons can be made on modcls articulated at the base and suitably acljusted for damping and period of vibration.

The separation of wind effects into a mean component and a superimposed fluctuating component made the analysis and the comparison between model and full scale much easier to understand. It was necessary to apply a correction to the reference static pressure for the mean component of the pressure, and the difference in time scales had to be taken into account in dealing with fluctuating components.

\subsection{Approximate Costs}

The part of the project involving measurements on the 34-story building covered the period from spring 1964 to approximatcly December 1967. The overall cost for the 4 ycars was approximatcly $\$ 100,000$. Of this, instrumentation, including installation, maintenance, and field trips accounted for $\$ 30,000$, engineers' and technicians' time about $\$ 60,000$, and computer processing at standard commercial rates $\$ 10,000$.

\section{Conclusion}

Wind pressure measurements are now under way in the building marked $\mathrm{A}$ in Figures 1 and 2. This is a 600 -ft high office building with a much more open exposure, particularly to the southwest. A new data acquisition system incorporating most of the suggested improvements has been installed at a cost of nearly $\$ 40,000$, and arrangements are being made to record the particle velocity of the top of the building. The main objective, as in the previous measurements, is the gathering of essential field data for the development and checking of wind tunncl techniques so that eventually they can be used with confidence for the determination of wind effects on buildings and structures.

Field measurcments on the 34-story office building could not have been made without the permission of the owners. The Division of Building Rescarch is grateful to Dorchester University Holdings Limited for their cooperation. Many pcople have contributed in various ways to the project; all are appreciated; in particular, the efforts of $\mathrm{Mr}$. W. von Tobel deserve special recognition.

The wind tunncl tests werc made at the University of Western Ontario Boundary-Laycr Wind Tunnel under the direction of Dr. A. G. Davenport and N. Isyumov. Their interest and assistance is gratefully acknowledged.
This paper is a contribution from the Division of Building Research, National Research Council, Canarla, and is published with the approval of the Director of the Division.

\section{References}

[1] Davenport, A. G. "Wind Loads on Structures." Division of Building Research, National Research Council, Canada, NRC 5576, March 1960.

[2] Jensen, M. "The Model-law for Phenomena in Natural Wind." Ingeniфren, International Edition, Vol. 2, No. 4, 1968.

[3] Newberry, C. W. "The Measurement of Wind Pressures on Tall Buildings." p. 113-150, NPI, Symposium on Wind Effects on Buildings and Structures, Vol. 1, London, HMSO, 1965.

[4] Newberry, C. W., K. J. Faton, and J. R. Mayne. "The Nature of Gust Loading on Tall Buildings." p. 339-428, Proc. International Research Scminar on Wind Effects on Buildings and Structures, Ottawa, Canada, September 1967, Vol. 1, University of Toronto Press, 1968.

[5] Newberry, C. W. and J. R. Mayne. "Wind Loading of a Tall Building in an Urban Environment; A comparison of Full Scalc and Wind Tunnel Tests." Paper 3, Symposium on Wind Effects on Buildings and Structures, April 1968, Loughborough University of Technology, Vol. 1, England, 1968.

[6] Joubert, P. N. et al. "The Drag of Bluff Boclies Immersed in a Turbulent Boundary Layer." p. 297-336, Proc., International Research Scminar on Wind Effects on Buildings and Structures, Ottawa, Canada, September 1967, University of Toronto Press, 1968.

[7] Van Koten, H. "Wind Measurements of High Buildings in the Netherlands." p. 685-704, Proc., International Research Seminar on Wind Effects on Buildings and Structures, Ottawa, Canada, September 1967, University of Toronto Press, 1968.

[8] Davenport, A. G. "The Application of Statistical Concepts to the Wind Loading of Structures." Proc., Institution of Civil Engincers, August 1961.

[9] "Tenantless Building Will Rise in Path of Typhoons." Engineering News Record, March 14, 1968, p. 29.

[10] Leutheusser, H. J. and W. D. Baines. "Similitude Problems in Building Aerodynamics." Journal of Hydraulics Division, Proc., American Society of Civil Engineers, p. 35-49, May 1967.

[11] Davenport, A. G. and N. Isyumov. "The Application of the Boundary Layer Wind Tunnel to the Prediction of Wind I oading." p. 201-230, Proc., Interuational Research Seminar on Wind Effects on Buildings and Struetures, Ottawa, Canada, September 1967, University of Toronto Press, 1968.

[12] Bendat, J. S. and A. G. Piersol. Measurement and Analysis of Random Data. New York, John Wiley and Sons, 1966.

[13] Lee, Y. W. Statistical Theory of Communication. New York, John Wiley and Sons, 1960.

[14] Rathbun, J. C. "Wind Forces on a Tall Building." Trans. American Society of Civil Engineers, Paper No. 2056, Vol. 105, p. 1-41, 1940.

[15] Dryden, H. L. and G. C. Hill. "Wind Pressure on a Model of the Empire State Building." Journal of Research, National Burcau of Standards, Vol. 10, p. $493-$ $523,1933$. 



\title{
INFLUENCE OF ARCHITECTURAL FEATURES ON THE S'TATIC WIND LOADING OF BUILDINGS
}

\author{
Hans J. Leutheusser \\ Department of Mechanical Engineering \\ University of Toronio \\ Toronto 5, Ontario, Canada
}

\begin{abstract}
The paper deals with the exploration, by model tests, of the effects of roof parapets and roof projections on to the roof wind-loading, and of wall mullions (or ribs) onto the wall wind-loading, of bluff building forms. Results are presented which suggest that parapets are effective in equalizing the pressure distribution over the roof surface while slightly reducing the uplift force. Roof projections, on the other hand, have the opposite effects. The presence of wall mullions causes sawtooth like deviations from the peripheral wall pressure-distribution for the no-rib case. The deviations are particularly significant in regions of attached flow and tend to become more pronounced with an increase in rib spacing.
\end{abstract}

Key words: Architectural features; buildings; mullions; parapets; roof projections; static pressures; wind effects; wind tunnel modeling.

\section{Introduction}

For the majority of conventional buildings knowledge of the time-averaged, or static, wind loading (as opposed to the dynamic loading due to vortex shedding and turbulence) is usually sufficient to allow adequate design of exterior walls, roofs and cladding, and to test the stability of a structure as a whole against overturning. Because of its great utility, hence, information on the static wind loading is usually summarized for a wide range of building configurations in the building codes of most countries.

The bulk of these code data originates from model tests which are performed, with various degrees of sophistication, in wind or water tunnels of laboratories specializing in experimental building aerodynamics. Since the physical size of models in relation to their full-scale counterparts is very small (linear scale ratios of $1: 200$ to $1: 400$ are common) it proves rarely possible to reproduce in a model with sufficient detail all of the architectural features of a prototype structure which would assure exact geometric similarity between the two. As a consequence, it has long been recognized that models may be inadvertently oversimplificd and, hence, that tests may yield incompletc or even misleading results.

In order to ascertain the influence which architcctural features might have on the static wind loading of buildings a number of systematic investigations have been performed in the Fluid Mcchanics Laboratory of the Department of Mechanical Enginecring, University of Toronto. In particular, tests were undertaken to study the effects of roof parapets and roof projections onto the roof wind-loading of flat-topped, block-type structures and circular cylinders. Furthermore, an investigation has now been initiated to cxplore the cffects of wall mullions (or ribs) on the peripheral wall pressure-distribution of very tall structures of rectangular plan. This latter study is still in progress, but some results for the square-plan building configuration arc already available. All of the various investigations, which are described in some detail in the following, were stimulated by the keen interest taken in the subject matter by the Division of Building Research of the National Research Council of Canada.

\section{Investigative Procedure}

Wind action on buildings and structures is a consequence of what in Fluid Mechanics is referred to as flow about bluff bodies at large Reynolds number. This statement implies that the flow field can be divided into a potential flow zone, a boundary-layer region and a turbulent wake. Although the field can be described by equations of motion, the complexity of them precludes mathematical solution. Hence, the experimental approach must be adopted for the solution of most practical problems such as the determination of the static wind loading on buildings.

The investigations under consideration here were variously carried out in one of two essentially identical micrometcorological wind-tunnel facilities. These tunncls are of the opcn-return typc, have test sections $8 \mathrm{ft}$ wide, $4 \mathrm{ft}$ high and $36 \mathrm{ft}$ long and arc cquipped with continuously variable speed 
drives at their downstream ends. At its upstream end, each tunnel contains a honeycomb followed by a stilling chamber with screens and a gradual constriction. The maximum air speed realizable in the two tunnels is, respectively, about 25 and about $60 \mathrm{ft}$ per second.

All tests were performed with the models installed in the uniform velocity field of the potential flow core of the wind-tunnel test-section. This was done although it is well recognized that this classical experimental technique can yield results of only limited practical utility [1].* However, the method was judged to be applicable in the present instance since the main concern of the various studies was to explore relative changes. In other words, the investigations were based on the premise that relative effects on pressure distributions brought about by minor modifications in building geometry are sensibly independent of the mean flow and turbulence characteristics of the velocity field.

Throughout the testing program special precautions were taken to effectively seal the leeward, low-pressure, building wake against falsifying entrainment of ambient-pressure fluid [1]. This was accomplished by using ground and separating plates of sufficient size, and by carefully blocking all possible leakage paths between high and low pressure zones on the models.

For the purpose of determining the pressure distribution, selected regions of the model exteriors were provided with closely spaced and carefully finished static pressure taps. Local pressurcs $(p)$ were determined with respect to the ambient static pressure $\left(p_{o}\right)$ prevailing in the field of undisturbed flow velocity $\left(v_{0}\right)$ immediately upstream of the model, as indicated by the static holes of a strategically arranged Pitot-static tube. Pressure differences evaluated from the readings of a sensitive micromanometer were rendered nondimensional by division with the dynamic pressure of the undisturbed airstream to yield nondimensional local pressure coefficients, viz.

$$
c_{p}=\frac{p-p_{o}}{\rho v_{o}^{2} / 2},
$$

where $\rho$ is the fluid density. Average pressure coefficients were deduced by numerically integrating the local pressure coefficients over appropriate building surfaces, i.e.,

\footnotetext{
* Figures in brackets indicate references at the end of paper.
}

$$
\overline{c_{p}}=\frac{\sum\left(c_{p} \Delta A\right)}{A}
$$

where $A$ is the total area, and $\Delta A$ is the area element contributory to an individual pressure tap.

\section{Study of Roof Parapets}

\subsection{Scope and Experimental Details}

A very common feature of flat-roofed buildings is the parapet, a low wall around the edges of the roof. Parapets are usually integral parts of the outside walls of buildings and are designed to provide positive anchorage of roof to structure. However, as first suggested by Nacy [2], parapets may also be beneficial in reducing local extremes of wind suction by equalizing the wind pressure distribution over the roof surface. Since the occurrence of local pressure extremes, or more specifically, of the associated steep pressure gradients, often entails wind damage to cladding, parapets would thus appear to represent an appealing combination of accepted architectural feature and wind safeguard.

In an effort to explore the promising characteristics of parapets in some greater detail, a study program was devised encompassing representative ranges of both building configuration and parapet height [3]. The general configurations of the building models investigated are illustrated in the definition diagrams of Figure 1. In particular, for the block-type structure, the aspect ratio of the building plan $b / l$ was either $1 / 2$ (oblong) or 1 (square), and for both the block-type and the cylindrical structures the relative building height $h / b($ or $h / d)$ varied between $1 / 2,1$, and 2 . Similarly, the relative parapet height $p / h$ ranged from 0 to $1 / 6$ for both building configurations.

The models were manufactured from clear acrylic plastic at an actual size of $b=d=6 \mathrm{in}$. and with a uniform parapet thickness of $1 / 8$ in. Each of the three basic building shapes (i.e. oblong, square and round) comprised three main components, viz. three interchangeable wall shells, five interchangeable parapet rings, and the roof proper which was provided with pressure taps. The top photograph of Figure 2 represents an "exploded" view of the oblong block-type structure, and the bottom photograph shows this structure mounted on a ground plate and installed in the wind tunnel.

All tests were performed at a free-stream velocity $v_{o}$ of about $20 \mathrm{ft}$ per second (corresponding to a Reynolds number, based on the minimum model dimension $b$ or $d$, of about $6 \times 10^{4}$ ), and each building 


\section{Definition Diagram of ROOF PARAPETS}

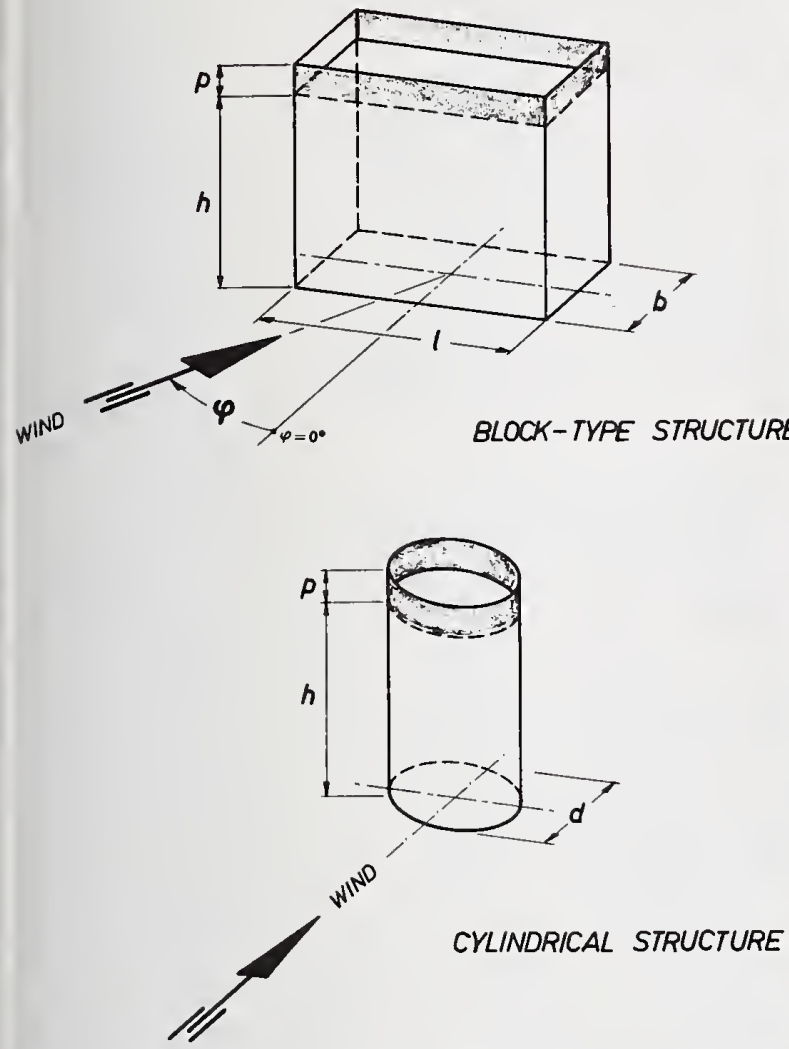

FIGURE 1. Definition diagram of roof parapets.

configuration was tested at all of the principal wind directions.

\subsection{Results and Discussion}

By way of example, figures $3 \mathrm{a}$ and $3 \mathrm{~b}$ depict experimentally determined distributions of nondimensionalized relative pressure over the roof surface of the square-plan block-type structure $(h / b=1)$ for six values of relative parapet height and for the two principal wind directions applicable in this case. The information conveyed by the various plots is representative of the sum total of general findings of the investigation which can be summarized as follows:

In general, the nonuniformity of pressure distribution is greatest for a $p / h$-ratio equal to zero. Increasing the height of parapets tends to equalize the pressure over the roof surface. This effect becomes more pronounced with an increase in building height.

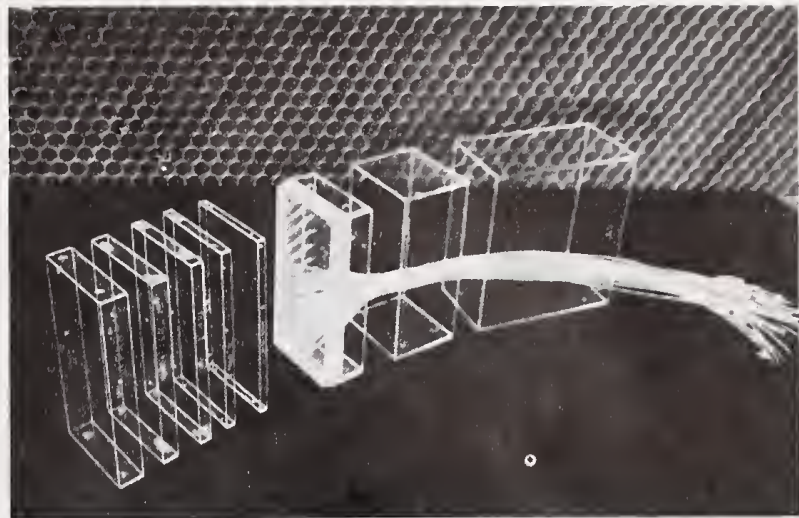

Complete Set of Model Componets of Structure with $b / \tau=1 / 2$

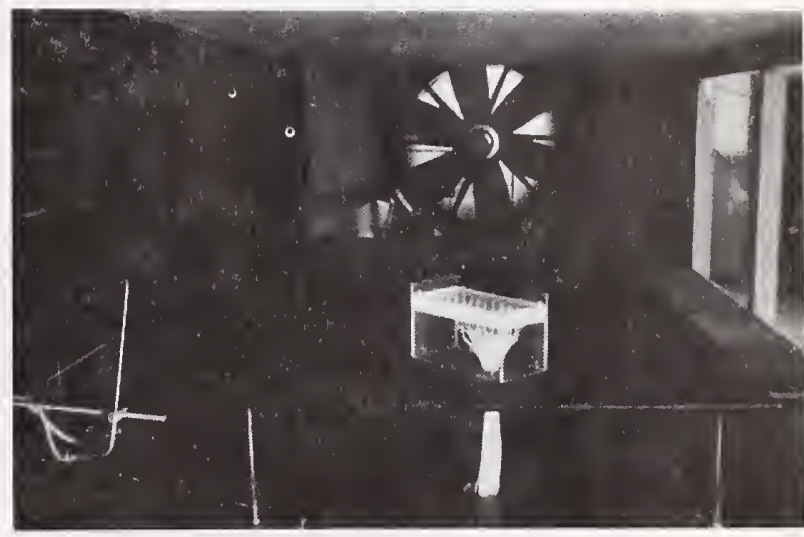

Model Installed in Wind Tunnel $\left(\mathrm{b} / \tau=1 / 2, \mathrm{~h} / \mathrm{b}=1 / 2, \mathrm{p} / \mathrm{h}=1 / 6, \varphi=45^{\circ}\right)$

Figure 2. Parapet study, model components and model installation.

For block-type structures, $45^{\circ}$ winds result in large negative pressures close to the leading corner. In the case of low buildings, parapets of small height make these pressures even more severe. Similarly, these extreme negative pressures increase with decreasing aspect ratio $b / l$ of the building plan.

Addition of parapets may result in some instances in locally positive pressures. These will occur mainly in leeward roof areas of low block-type structures equipped with high parapets and subjected to cornering winds.

A more concise summary of the test results than can be provided by plots of isobars is offered in Figure 4 . Here the effectiveness of parapets in relation to pressure equalization is expressed in terms 
Archit'l Feature: ROOF PARAPETS ISOBARS ( $c_{p}$-Contours) on ROOF BLOCK-TYPE STRUCTURE

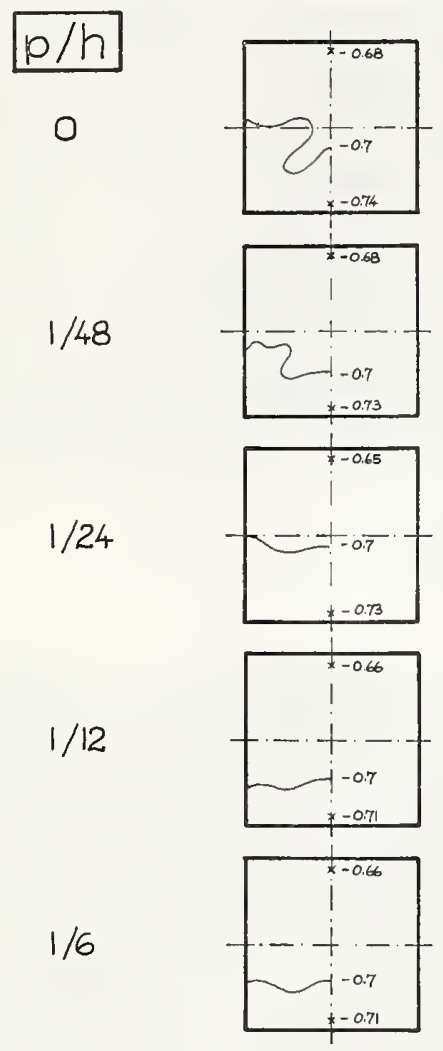

Figure 3a. Effects of parapets on roof pressure-distribution for frontal wind.

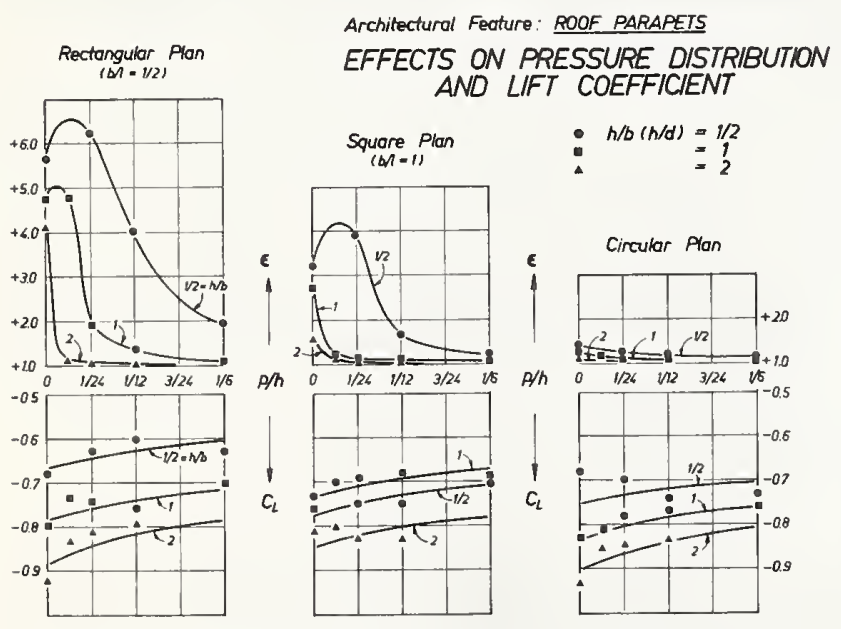

Figure 4. Neteffects of roof parapets on pressure distribution and on lift coefficient.

of an index (tending toward unity for complete uniformity)
Archit'l Feature: ROOF PARAPETS

ISOBARS ( $c_{p}$-Contours) on ROOF

BLOCK-TYPE STRUCTURE

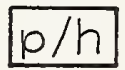

○
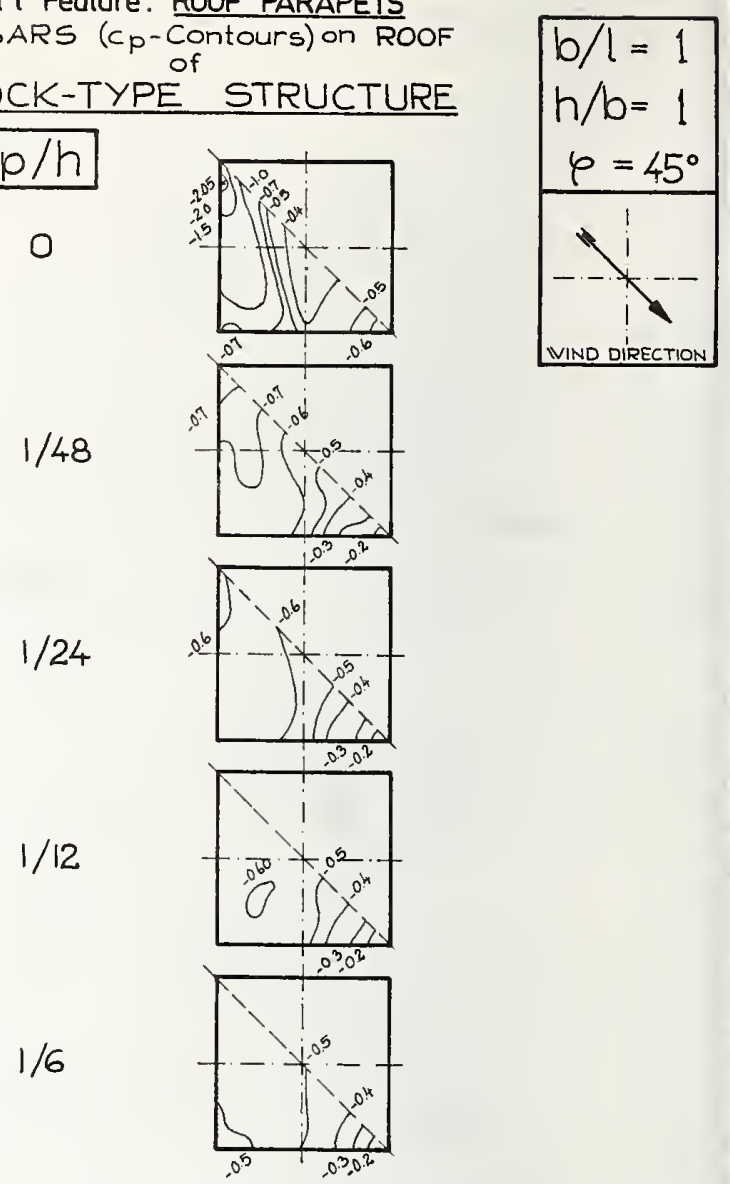

Figure 3b. Effects of parapets on roof pressure-distribution for cornering wind.

$$
\epsilon=\frac{\left(c_{p}\right)_{\mathrm{extr}}}{C_{L}}
$$

in which $\left(c_{p}\right)_{\text {extr }}$ is the largest measured local pressure coefficient of a particular pressure distribution, and $C_{L}$ is the lift coefficient or, what is the same in this case, the average pressure coefficient for the whole roof as defined by Eq. 2, where $A$ is the total roof area inside of the parapet. Since for design purposes, at least, distinction between wind directions is irrelevant, both $\epsilon$ and $C_{L}$ are plotted in Figure 4 as function of relative parapet height $p / h$ with relative building height $h / b($ or $h / d)$ as a parameter, but irrespective of wind angle. As a consequence, the curves in Figure 4 actually represent the envelopes of the most critical values of $\epsilon$ and $C_{L}$ for all of the wind directions tested.

The systematic variation of $\epsilon$ in Figure 4 is remarkable, particularly in view of the somewhat more erratic behavior of the corresponding lift coefficients. It is immediately apparent that 
for all structures not only relative parapet height but also relative building height has a pronounced effect on the nonuniformity of pressure distribution as measured by $\epsilon$. The latter decreases in general with increasing values of $p / h$ and $h / b$ (or $h / d$ ). As suggested also by the isobar plots, addition of parapets of insufficient height to low block-type structures may cause the nonuniformity of pressure distribution to become more severe. It is noteworthy that this effect is particularly pronounced in the case of the oblong block-type structure. It is also apparent that, for the purpose of pressure equalization, parapets are not likely to be very useful on tall block-type structures and on cylindrical structures of any height.

The variation of lift cocfficient with relative parapet height depicted in Figure 4 shows a generally decreasing trend for all three types of structure and for all building heights. Over the range of relative parapet heights tested, this load relicving effect of parapets increases to about $10 \%$ of the uplift force on an unprotected roof (i.e., one for which $p / h=0$ ). It is also apparent that lift coefficients becomc numerically larger with an increase in building height whether or not parapets are present. Indeed, from the data available it is clear that it is relative building height rather than relative parapet height which is the significant parameter in determining the applicable roof lift coefficient for a particular building. However, somewhat inconclusive experimental evidence [3] suggests that the lift coefficient will become independent of relative building height at values of $h / b$ (or $h / d$ ) of about 10, in which case then relative parapet height would assume the role of governing parameter.

\section{Study of Roof Projections}

\subsection{Scope and Experimental Details}

The term "projections" refers to those portions of the roof which overhang the side walls of a building. Roof projections are a normal and, indeed, necessary feature of practically all residential buildings insofar as they serve to protect outside walls against the direct roof runoff of precipitation, and provide shade. Even clementary aerodynamic considerations suggest that roof projections will have an adverse effect on the static wind loading of both roof and walls, particularly in the case of flat-topped buildings. However, no concerted attempt at clarifying their role seems to have ever been made.
In an attempt to provide some quantitative information on the influence of projections onto the roof wind-loading of flat-topped buildings, a study program similar in scope to that of the previously reported parapet study was exceuted [4]. The general configurations of the various building models investigated are depicted in Figure 5. As before, the aspect ratio $b / l$ of the plan of the block-type structures was either $1 / 2$ (oblong) or 1 (square), and the relative building height $h / b$ (or $h / d$ ) varied between $1 / 2,1$, and 2 . Similarly, the relative roof projection $e / h$ ranged in uneven increments between 0 and $1 / 6$.

The models were manufactured out of plastic and built at an actual size of $b=d=6$ inches. The overhanging portions of the roofs had a uniform thickness of $3 / 16$ inch. Each of the three basic building shapes, i.e., oblong, square, and round, comprised two main components, viz. threc interchangable

\section{Definition Diagram of ROOF PROJECTIONS}
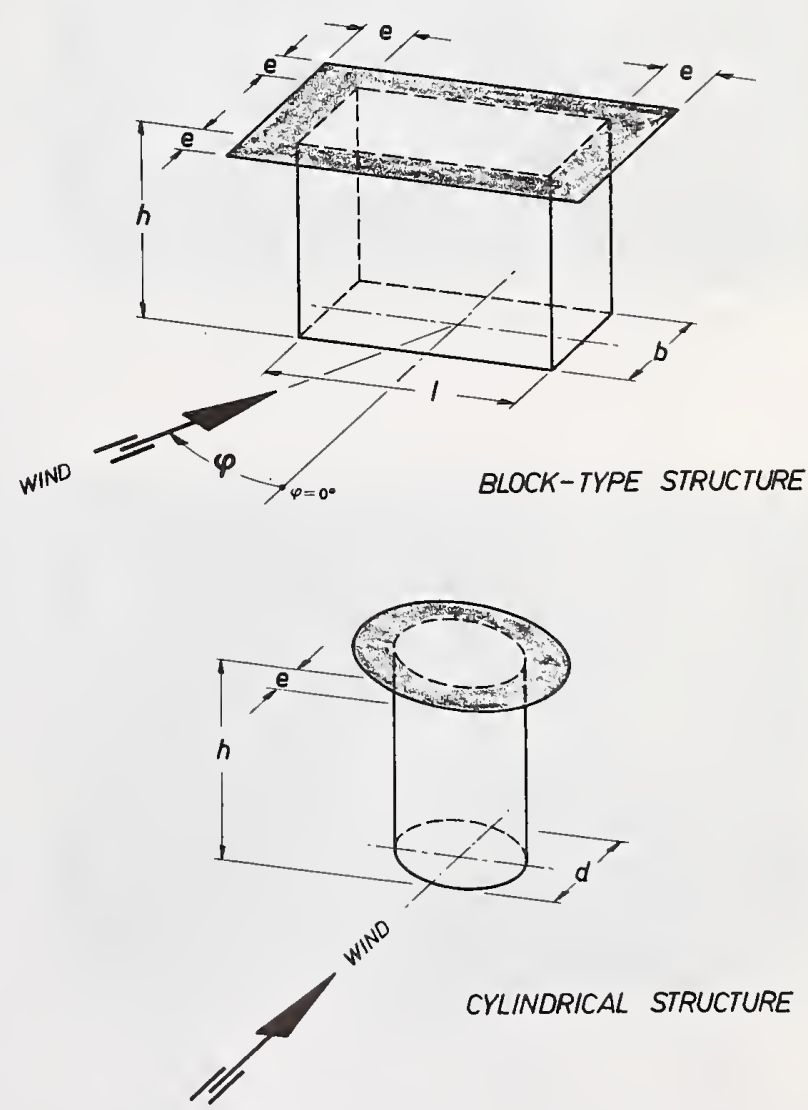

CYLINDRICAL STRUCTURE

Figure 5. Definition diagram of roof projections. 
wall shells and four intercliangable roofs. Each roof consisted of three laminated layers of sheet plastic. The bottom layer was $1 / 4$-inch thick, fitted into the appropriate wall shell and accommodated the manometer-lead receptacles for pressure taps strategically arranged in bow the top surface of the whole roof and the underside of the roof projections. The $1 / 8$-inch thick center layer and the 1/16-inch top layer together constituted the roof proper. Its two-layer construction was necessitated by the requirement for having internal connections between the pressure taps in the projecting portions of the roof and their corresponding manometer-lead receptacles in the interior of the model. The internal connections were realized by milling shallow channels into the upper surface of the center layer of the roof, and by

\section{MODEL STUDY OF ROOF PROJECTIONS}

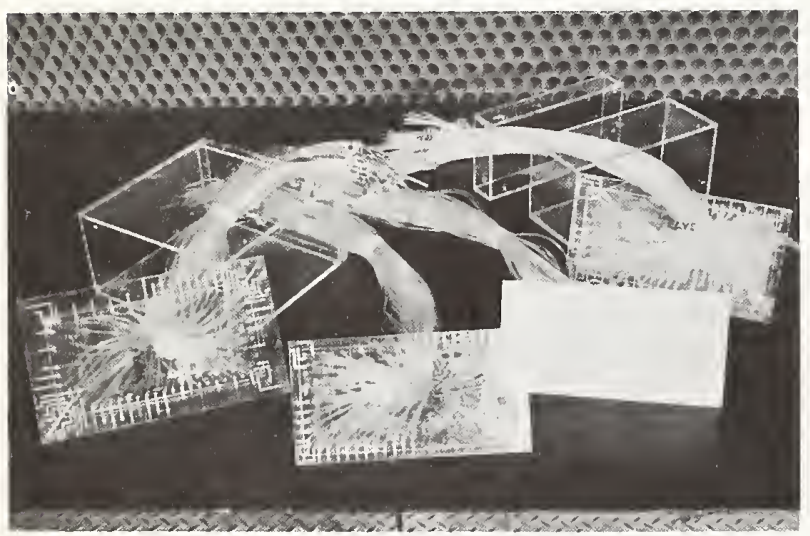

Complete Set of Model Components of Structure with $b / \tau=1 / 2$

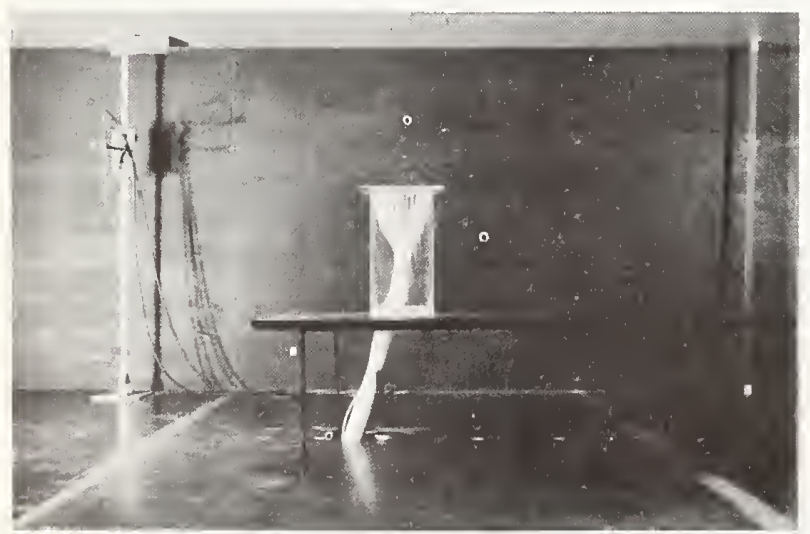

Model Installed in Wind Tunnel $\left(b / \tau=1, h / b=2, e / h=1 / 12, \varphi=0^{\circ}\right)$

Figure 6. Projection study, model components, and model installation. subsequently cementing the top layer to this surface. Static pressure holes were drilled after the three layers had been laminated together, and all internal channels, as well as all straight-through taps, were carefully pressure-tested for cross-leakage. By way of example, the top photograph of Figure 6 portrays the complete complement of components for the oblong block-type structure, and the bottom photograph shows the square-plan block-type structure installed in the wind tunnel.

As in the case of the parapet study all tests were run with a free-stream velocity of about 20 feet per second, and each building configuration was tested at all of the principal wind directions.

\subsection{Results and Discussion}

Figures $7 \mathrm{a}$ and $7 \mathrm{~b}$ show the pressure distributions over the upper and lower roof surfaces of the squareplan block-type structure $(h / b=1)$ for six values of relative roof projection and for the two principal wind directions applicable to this building shape. These diagrams are representative examples of all of the isobar plots prepared in the course of the data evaluation.

Inspection reveals that an increase in relative roof projection causes the pressure distributions to become more nonuniform, particularly for frontal winds. However, the changes do not appear to be very drastic, at least not for the range of $e / h$-values studied. As would be expected, $45^{\circ}$ winds acting on the block-type structures give rise to local suction extremes on the top surface of the roof in the immediate vicinity of the leading corner. To be sure, the measured values of these pressure extremes for the various $e / h$-values pertaining to a particular combination of $b / l$ and $h / b$ do not permit direct comparison because the members of such a series of models are not geometrically similar. However, in overall view of the experimental evidence available it would appear that also local suction extremes ought to be relatively insensitive to changes in $e / h$.

Of particular interest are the pressure distributions over the underside of roof projections. Positive pressures prevail generally over all eaves overshadowing windward building walls. In the case of cylindrical structures the positive pressure region is restricted to the upwind $60^{\circ}$ sector. The balance of the eaves is subject to relatively weak suction as indicated by measured negative pressure coefficients which nowhere exceed an absolute value of 0.9 .

Integration of the various pressure distributions over the roof surfaces of all structures tested showed 
Architectural Feoture: ROOF PROJECTIONS

ISOBARS ( $c_{p}$-Contours) on ROOF of

BLOCK-TYPE STRUCTURE

TOP SURFACE
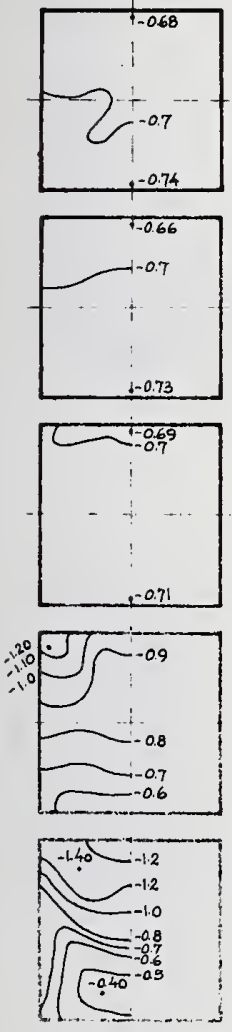

$1 / 48$

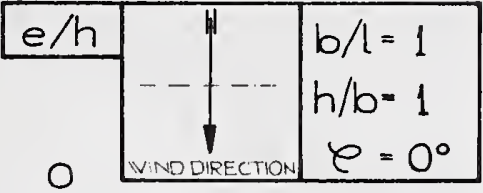

(as seen from above)

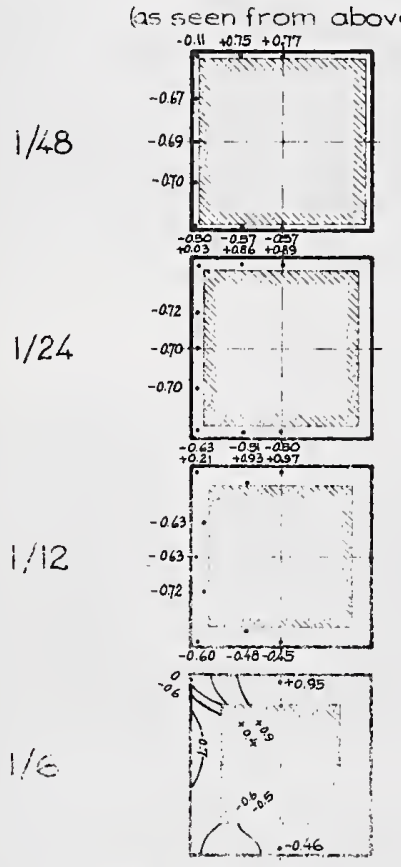

FigURe 7a. Effects of projections on roof pressure-distribution for frontal wind.

that both the average pressure coefficient $\left(\overline{c_{p T}}\right)$ for the top surface of the roof $\left(A_{T}\right)$, and the average pressure coefficient $\left(\overline{c_{p U}}\right)$ for the underside of the roof projection $\left(A_{U}\right)$ are invariably negative, i.e. that there is always an upward directed uplift force acting on the top surface, and a downward directed downpull force acting on the eaves. As a consequence, the net lift coefficient for the whole roof, i.e.,

$$
C_{L}=\frac{L / A_{T}}{\rho v_{0}^{2} / 2}=\overline{c_{p T}}-\overline{c_{p U}}\left(A_{U} / A_{T}\right)
$$

where $L$ is the net lift force, is found to vary little and, indeed, may even tend to decrease with an increase in relative roof projection.

However, by its very definition $C_{L}$ is not a direct measure of the net uplift force aciing on the roof of a given structure since it depends on $A_{T}$ and, hence, on $e / h$. More meaningful for judging the effects of variable relative roof projection on the net lift force is the "specific" lift coefficient defined as
Architectural Feature: ROOF PROJECTIONS
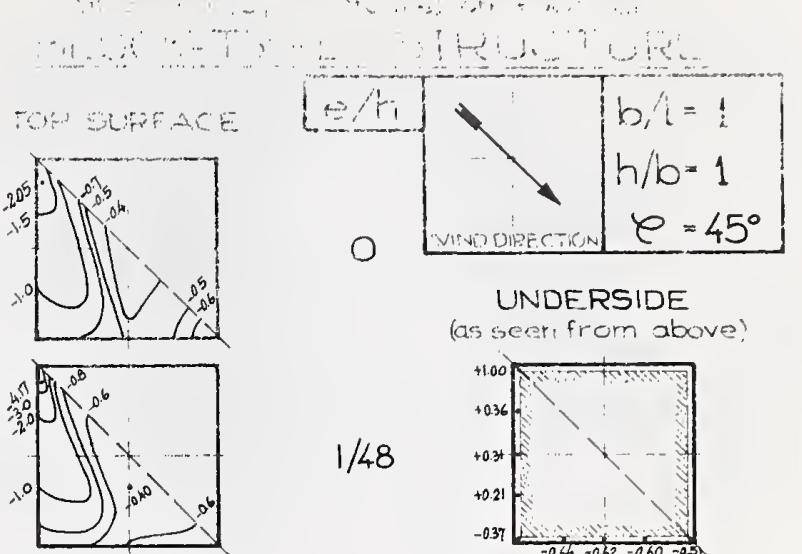

$1 / 48$
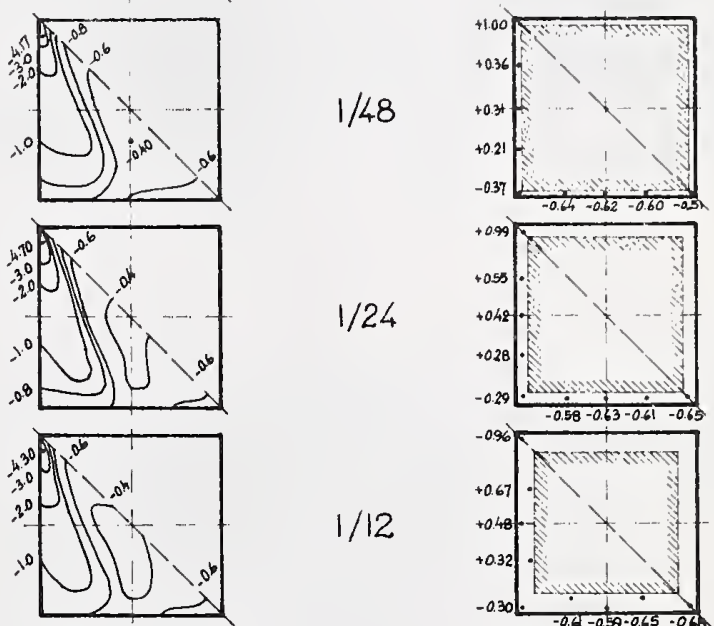

$1 / 24$

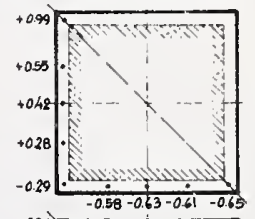

$1 / 12$

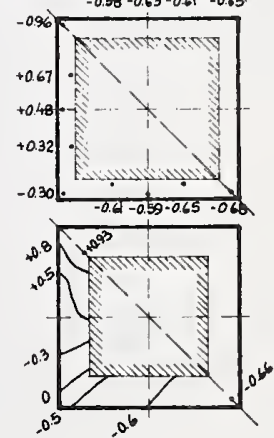

Figure 7b. Effects of projections on roof pressurc-distribution for cornering wind.

$$
C_{L}^{+}=C_{L}\left(A_{T} / A^{+}\right)=\frac{L / A^{+}}{\rho v_{0}^{2} / 2}
$$

which is referred to $A^{+}$, the plan area (i.e., $b \times l \mathrm{or}^{\circ}$ $\left.d^{2} \pi / 4\right)$ of the structure proper. The variation of the maximum (i.e., irrespective of wind direction) specific lift coefficient as a function of $e / h$ with $h / b$ (or $h / d)$ as a parameter is plotted in Figure 8 . Inspection of the curves leads to the interesting conclusion that the net uplift force on roofs of cylindrical structures is, over a certain range of the argument, practically independent of $e / h$ and, hence, of the roof diameter. The range is longest for the lowest structure and decreases quite systematically with increasing building height. On the other hand, only the two lowest of the block-type structures exhibit a similar characteristic, but the behavior is less pronounced particularly in the case of the oblong configuration. It is further apparent from Figure 8 that for all of the 


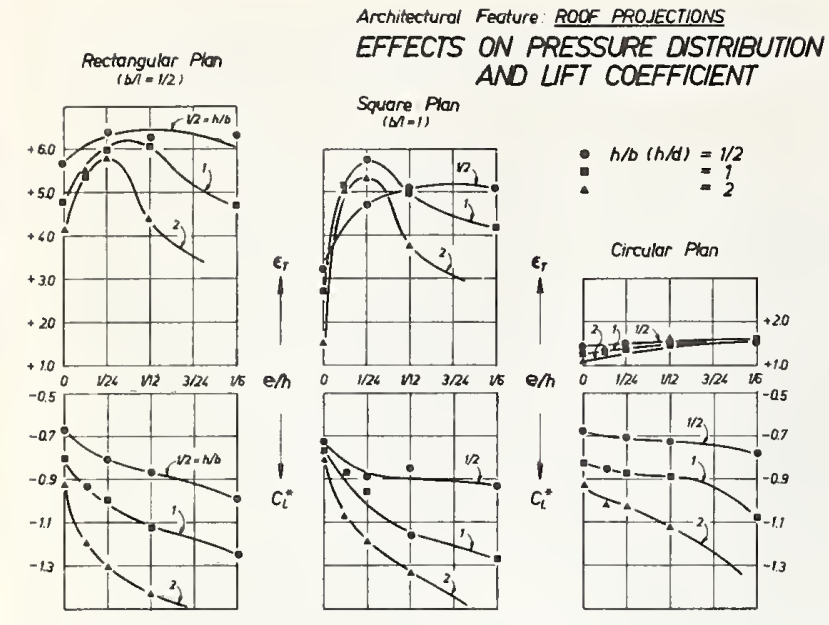

Figure 8. Net effects of roof projections on top-surface

building shapes investigated, $C_{L}+$ (and hence, the net uplift force) increases strongly with building height.

Also plotted on Figure 8 is an index illustrating the degree of nonuniformity existing on the top surface of the roof. This index is defined similar to Eq. 3, i.e.,

$$
\epsilon_{T}=\frac{\left(c_{p T}\right)_{\text {extr }}}{\overline{c_{p T}}}
$$

where $\left(c_{p T}\right)_{\text {extr }}$ is the largest measured local pressure coefficient on the top surface. The variation of $\epsilon_{T}$ with $e / h$ and $h / b$ (or $h / d$ ), though rather erratic, certainly suggests that no benefit can be derived from roof projection with regard to pressure equalization.

\section{Study of Wall Mullions}

\subsection{Scope and Experimental Details}

Mullions, or vertical ribs, protruding from the exterior surface of outside building walls seem to become increasingly popular with architects designing high-rise office towers and apartment buildings. More often than not these ribs have no functional purpose and are used strictly for appearance's sake. Some limited information on the pressure distribution over the outside walls of cylindrical tanks with vertical fins [5] suggests that there may occur significant pressure differences across wall projections and, hence, that mullions may be subjected to possibly very large acrodynamic forces due to wind action. However, no pertinent design data which would be directly applicable to angular buildings with plane walls appears to be presently available in the literature.
In an effort to at least partially close this information gap a study program was devised around the concept of a very tall, i.e., essentially two-dimensional, building of rectangular plan configuration equipped with mullions of identical cross-section geometry but of variable spacing between fixed corner arrangements.

The general characteristics of this building are indicated in Figure 9. Although the model of this structure permits changing the aspect ratio $b / l$ of the building plan from $1 / 2$ (oblong) to 1 (square), only tests on the square-plan configuration have so far been undertaken. Similarly, the model is actually designed in such a way that a wide variety of types of ribbed building walls can be studied. However, during the investigation here under consideration only mullions of square plan arranged with variable spacings $s / b$ of $1 / 27,3 / 27$ and $5 / 27$ have been used.

The square-plan model tested has an actual physical size of $b=13.5$ inches. It consists of four main components, viz. model stand, one set of (square) end plates, one set of separating plates (attached to the end plates and serving as wake seals), and four sets of wall panels. Each set of wall panels (three provided with ribs at different spacings and one without ribs for the purpose of comparison)

\section{Definition Diagram of WALL MULLIONS}

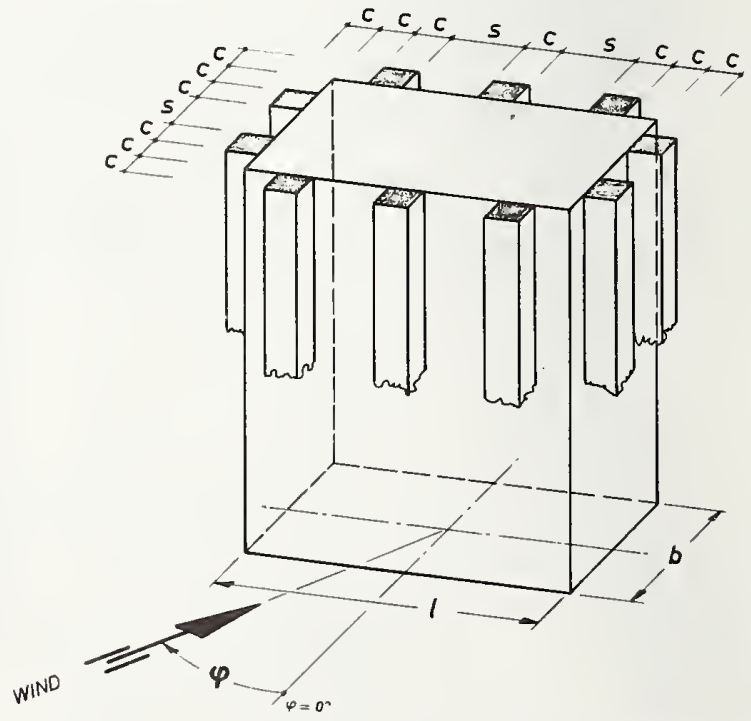

BLOCK-TYPE STRUCTURE

Frgure 9. Definition diagram of wall mullions. 


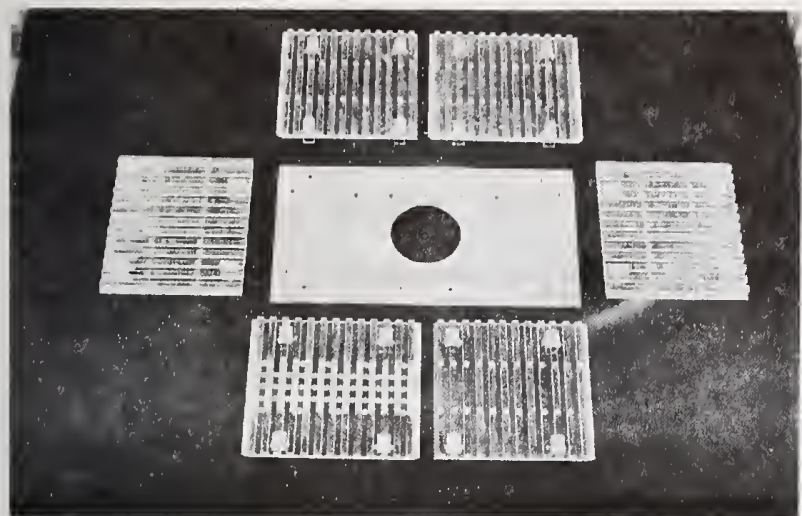

Complete Set of Model Walls with $s / b=1 / 27$

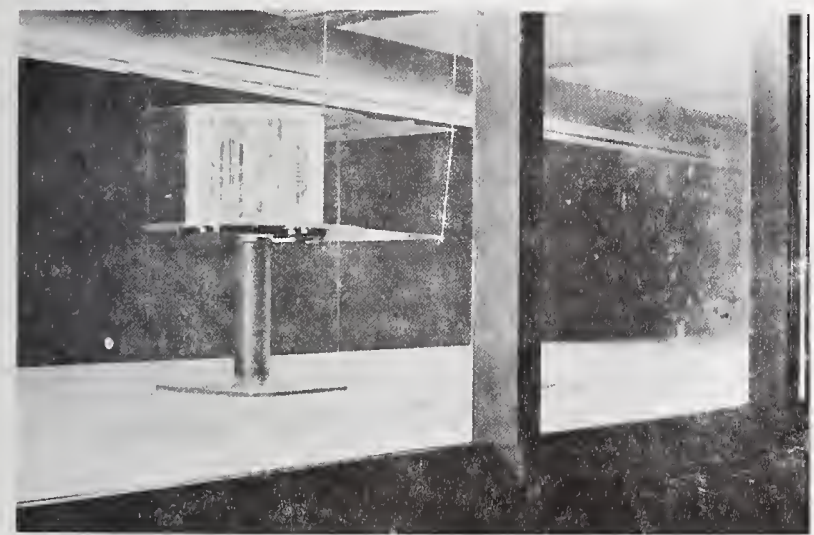

Model installed in Wind Tunnel $\left(b / \tau=1, e / b=1 / 27, s / b=1 / 27, e=45^{\circ}\right)$

Figure 10. Mullion study, model components and model installation.

comprise four panels of which one only is provided with pressure taps.

The arrangement of the pressure taps is staggered over the center third of the 12-in. high model, and there are a minimum of three taps in each half-inch of peripheral length including each mullion face. Hook-up of the pressure taps to a bundle of manometer leads which is permanently installed in the hollow shaft of the model stand is effected by bolting a lead-terminal contact plate leak-proof against the interior surface of the tapped wall panel.

The top photograph of Figure 10 portrays a complete set of wall panels for the oblong structure surrounding one of the corresponding model end-plates, and the bottom photograph shows the square-plan

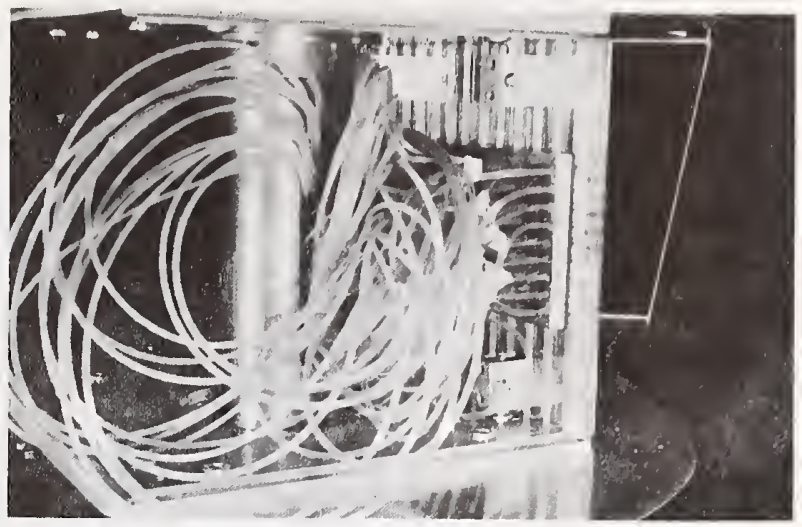

Model Interior Showing Contact Plate
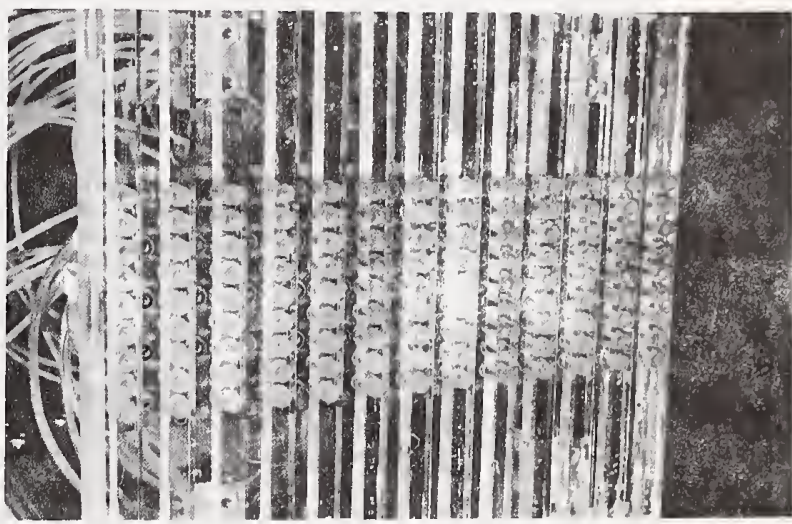

\section{Close-Up of Exterior Model Surface} $(s / b=1 / 27)$

Figure 11. Mullion study, model details.

model installed in the wind tumnel. Details of the pressure-tap hook-up, and the outside appearance of the tapped wall panel with the closest mullion spacing are shown in the two photographs of Figure 11.

All tests of the investigation were run at a freestream velocity of about $50 \mathrm{ft}$ per second (corresponding to a Reynolds number, based on the dimension $b$, of about $2.3 \times 10^{5}$ ), and each mullion configuration was tested for both frontal and cornering (i.e., $45^{\circ}$ ) winds.

\subsection{Results and Discussion}

In the order to assure two-dimensional flow about the model, the building wake had to be perfectly 

ON PERIPHERAL PRESSURE DISTRIBUTION
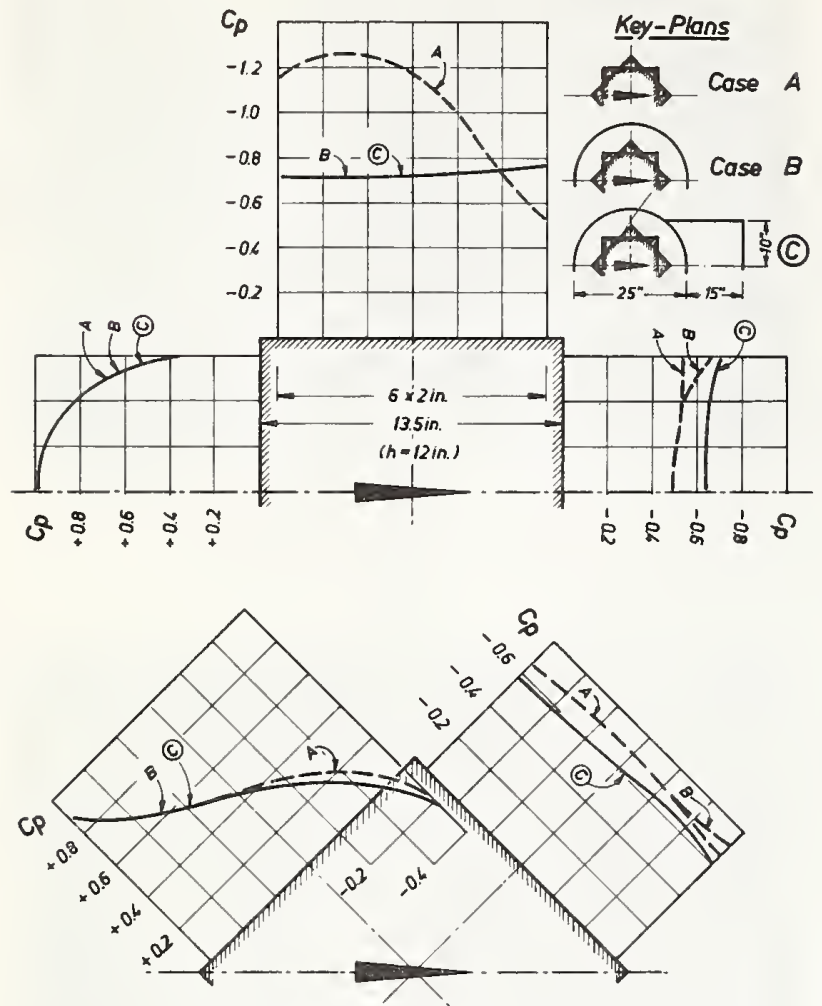

FIGURE 12. Pressure distribution on a square-plan cylinder.

sealed against inflow from above and below. Results of an incidental series of tests with various designs of separating plates attached to the plain structure are depicted in Figure 12. In this, cases A, B and C pertain to: absence of any plates; circular separating plates; and circular separating plates with tails; respectively. From a study of the flow behavior in the wake region with the aid of a tuft probe, case C was confirmed as providing complete wake sealing, and the corresponding distribution of peripheral wall pressure forms the basis of comparison to which are referred the results of the mullion study proper. Throughout this study the model was always equipped with separating plates corresponding to case C.

Figures 13a through $14 \mathrm{c}$ are plots of the nondimensional peripheral wall pressure-distribution on the square-plan structure for three cases of mullion spacing and two wind directions. In these, the solid lines correspond to pressures measured normal to the basic shell (i.e., $b \times l$ ) of the model, and the dots measure the average pressure difference across individual mullions. The latter type of data representation is made conveniently possible by the experimentally established fact that the pressure distribution over the mullion side-walls is essentially uniform.

Inspection of the various diagrams reveals that mullions may, indeed, cause serious modifications of the peripheral pressure distribution for the no-rib case shown as a dashed line. The modifications consist essentially of saw-tooth like deviations from the plain-wall case which increase in magnitude with an increase in mullion spacing. The effects of mullions on the pressure distribution are particularly significant in those regions of the building plan where, in the case of no ribs, the flow is attached to the structure. The static loading on mullions can be severe as indicated by the pressure conditions recorded for the windward corner shown in Figure 13c. Here the measurements indicate the existence of pressure differences in the neighborhood of twice the dynamic pressure in both directions across the mullion section. It appears, that still more critical conditions could result from larger mullion spacings than were tested so far.

\section{Conclusions}

True appreciation of the effects which architectural features might have on the static wind loading of buildings can only come through carefully performed model tests. While the paper summarizes a considerable body of hopefully useful information in this regard, the data presented actually pertain only to a very small number of features, viz. roof parapets, roof projections, and wall mullions. There are many other common architectural details which deserve to be studied in a similar systematic manner and much remains to be done before a really significant advance in the understanding of wind effects in this field of building aerodynamics will become apparent. 


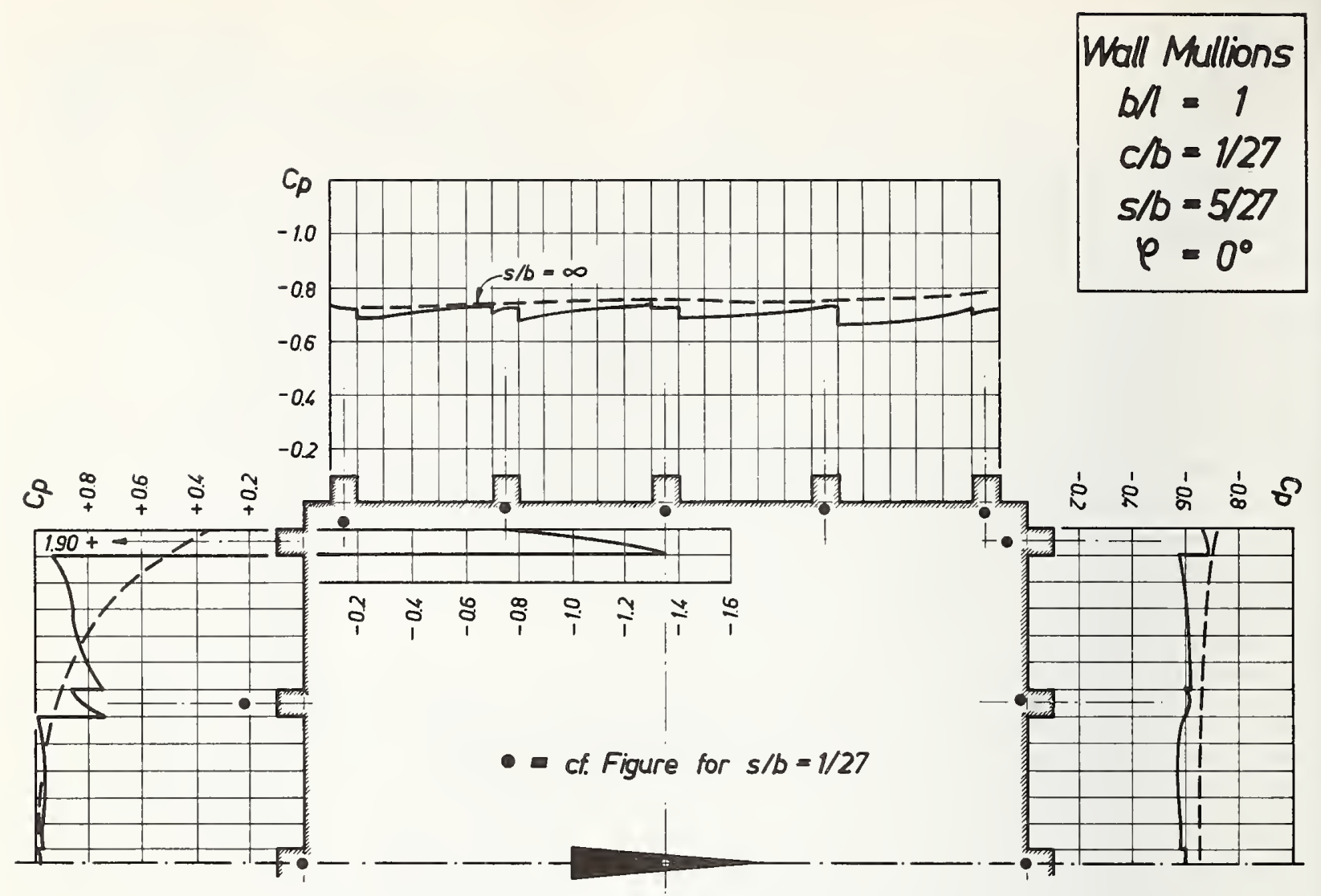

Figure 13c. Effects of wall mullions on peripheral pressure distribution for frontal wind $(s / b=5 / 27)$.

- Evg. loteral press. difference across mullions; positive if tending to turn clockwise

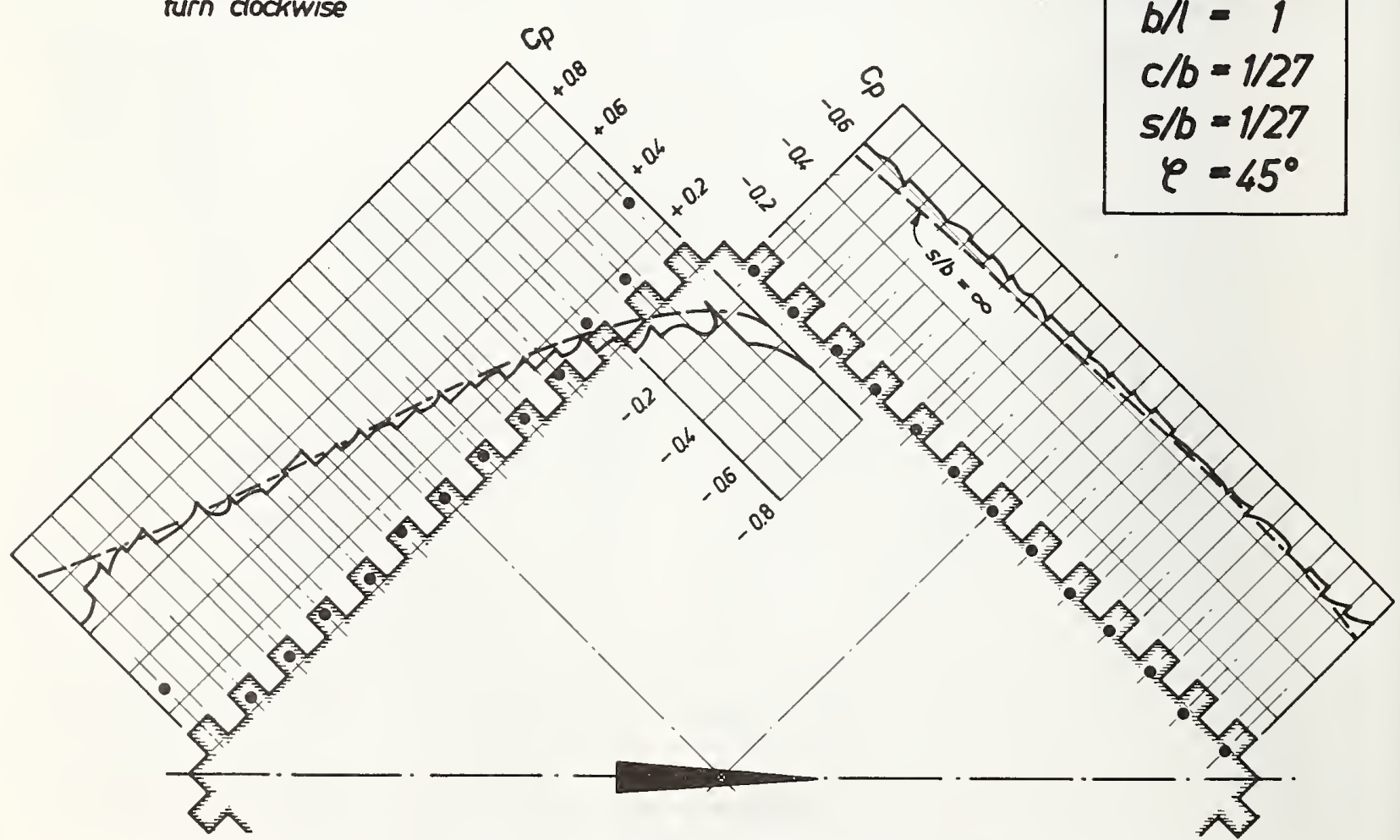

FIGURE 14a. Effects of wall mullions on peripheral pressure distribution for corncring wind $(s / b=1 / 27)$. 


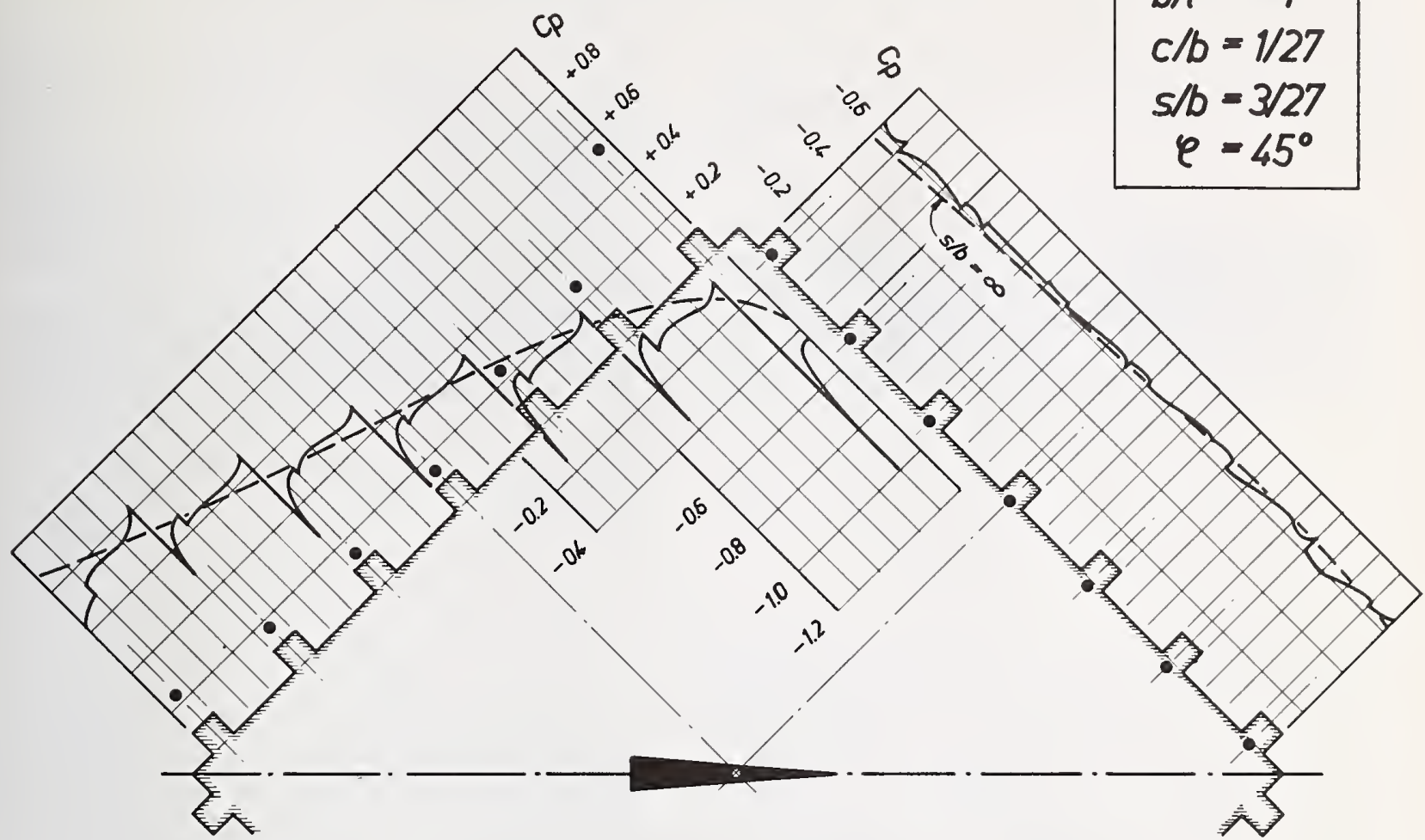

FIgURE $14 \mathrm{~b}$. Effects of wall mullions on peripheral pressure distribution for cornering wind $(s / b=3 / 27)$.

- of. Figure for $s / b=1 / 27$

Wall Mullions
$b / l=1$
$c / b=1 / 27$
$s / b=5 / 27$
$\varphi=45^{\circ}$

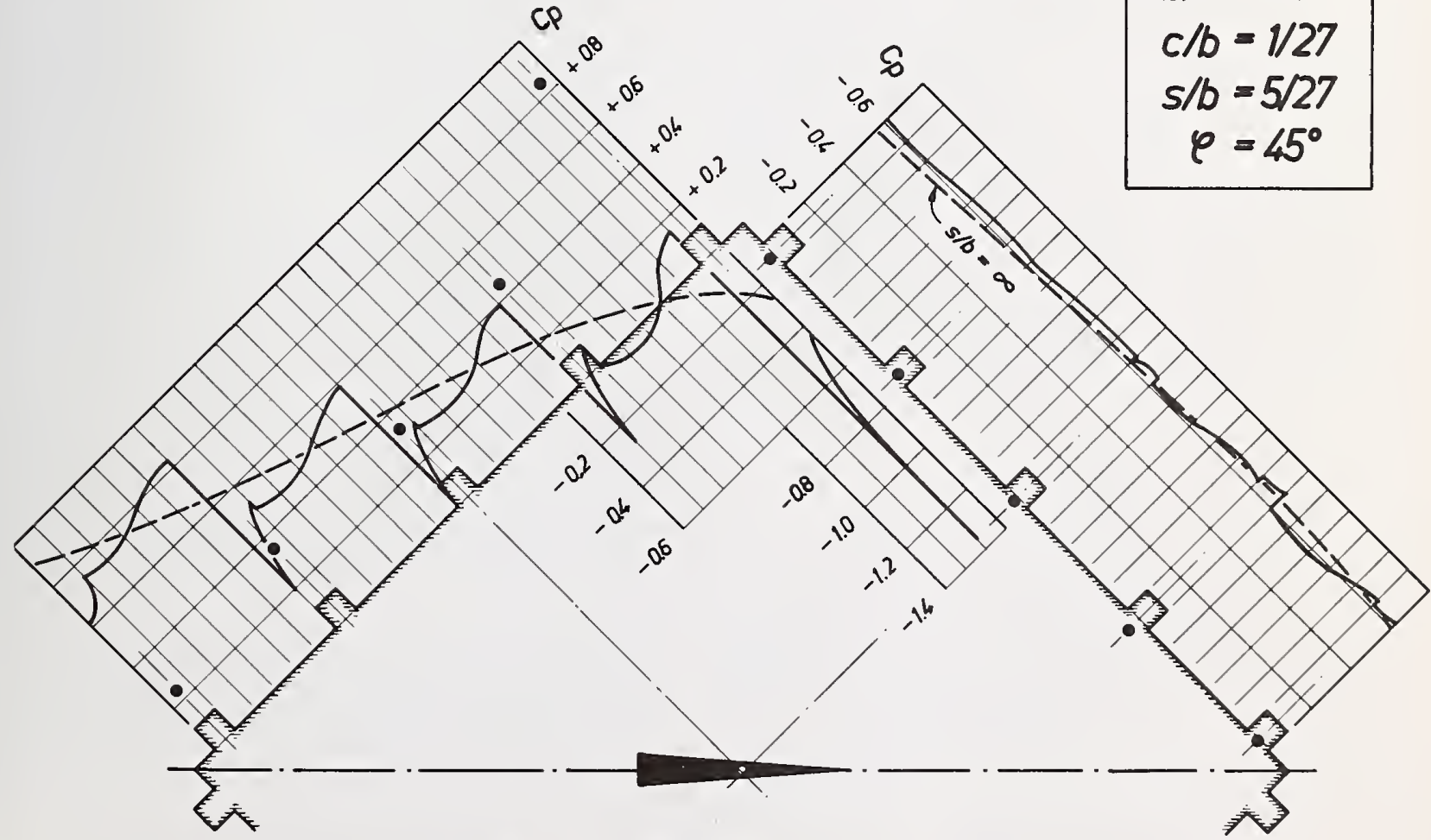

Figure 14c. Effects of wall mullions on peripheral pressure distribution for cornering wind $(s / b=5 / 27)$. 
The work described herein covers several years and was made possible through grants by the $\mathrm{Na}$ tional Research Council of Canada. The writer is indebted to his many co-workers who, at various times, rendered able assistance to his research program.

\section{References}

[1] Leutheusser, H. J. and Baines, W. D., "Similitude Problems in Building Aerodynamics," Journal of the Hydraulics Division, Proceedings of the American Society of Civil Engineers, Volume 93, No. HY3, May 1967.

[2] Nacy, P. S., "Modification of Pressure Distribution around Buildings due to Parapets," M.S.-Thesis, unpub- lished, Department of Mechanics and Hydraulies, Graduate College, State University of Iowa, June 1951.

[3] Leutheusser, H. J., "The Effects of Wall Parapets on the Roof Pressure-Coefficients of Block-Type and Cylindrical Structures," University of Toronto, Department of Mechanical Engineering, Technical Publication Series, TP 6404, April 1964.

[4] Leutheusser, H. J., "The Effects of Eaves on the Roof Pressure-Coefficients of Block-Type and Cylindrical Structures," University of Toronto, Department of Mechanical Engineering, Technical Publication Series, TP 6503, April 1965.

[5] Hoerner, S. F., "Fluid-Dynamic Drag, Practical Information on Acrodynamic Drag and Hydrodynamic Resistance," published by the author, Midland Park, N.J., 1965. 


\title{
THE UNSTEADY SURFACE PRESSURE AROUND CIRCULAR CYLINDERS IN TWO-DIMENSIONAL FLOW*
}

\author{
Wayne E. Simon \\ Martin Marietta Corporation \\ Denver Division \\ Denver, Colorado 80201
}

\begin{abstract}
The unsteady surface pressure around circular cylinders in two-dimensional flow has been measured in the subcritical, supercritical, and transeritical flow regions. A representation of the crosspower spectral density has bcen developed which is a reasonable representation of the data and which applies to all three regions. The most important result is the essential similarity of the subcritical and transcritical regions.
\end{abstract}

Key words: Circular cylinders; power spectra; pressure fluctuations; subcritical flow; supercritical flow; transcritical flow; turbulence.

\section{Introduction}

The original flow field parameter analysis was, in essence, a semiempirical representation of the unsteady aerodynamic force exerted on a boost vehicle in the vertical launch position. It was so constructed that the calculated response of the Saturn $\mathrm{V}$ wind tunnel model was a reasonably good representation of the wind tunnel data. In this work, it was found necessary to postulate a relationship between Strouhal frequency and the second derivative of the steady pressure at the separation point [1].**

An experimental program was then planned to investigate this postulate. The work reported in Chapter II, Section C of Reference 2 showed that this postulate was valid.

Another portion of the flow field parameter analysis postulated an exponential decay in the crosscorrelation of the unsteady force as a function of axial displacement. In addition, an effect of vehicle motion on the correlation length was also assumed. Although these assumptions were necessary to give a mathematical model that agreed with response measurements in the wind tunnel, data were not available to establish their validity. One fact indicated that some factor was still missing in the analysis. This fact was the observation that when

* This work was performed under Contract NAS8-5322, from the National Aeronautics and Space Administration, George C. Marshall Space Flight Center, Huntsville, Alabama.

** Figures in brackets indicate references at the end of this paper. the Strouhal frequency and the model frequency were the same, thus giving very high response, the observed motion of the model was approximately periodic and ncarly sinusoidal. The flow field parameter analysis computations gave a response of the right magnitude, but with a narrow-band random response that was not periodic.

Since the purpose of the flow field parameter analysis is to predict vehicle response based on data from a wind tunnel, it is very important that the analysis correetly reflects the physical situation.

An experimental program was then planned to investigate the unsteady surfaee pressure around a circular cylinder in two-dimensional flow. This program was designed to obtain data on the unsteady pressure for a relatively simple flow condition. To incorporate the unsteady pressure into the flow field parameter analysis, it will be necessary to obtain additional data for the effeet of three-dimensional flow (change of section and presence of the tip of the vehicle) and for the effect of vehicle motion on the unsteady pressure.

\section{Nomenclature}

$C_{P_{b}} \quad$ base pressure coefficient

$C_{D_{v}} \quad$ viscous drag coefficient

$q \quad$ free stream dynamic pressure

$F_{p_{1} p_{1}}(\omega)$ power spectral density of $p_{1}$

$F_{p_{1} p_{2}}(\omega)$ cross power spectral density of $p_{1}$ and $p_{2}$

$p \quad$ unsteady pressure

$\sigma_{p} \quad$ standard deviation of unsteady pressure $=$ RMS 


\section{Data Analysis}

The experimental program used two tunnelspanning cylinders (10 in. and 24 in. diameters) with smooth and rough surfaces and with low and high tunnel turbulence. It is then possible to produce subcritical flow by using the smooth 10-in. cylinder in a clean tunnel. Supercritical flow is produced either by using a smooth 10-in. cylinder with a turbulence grid in the tumnel, or by using the smooth 24 in. cylinder in a clean tunnel. Finally, transcritical flow is produced by using a rough 24-in. cylinder with a turbulence grid.

The first regime to be analyzed was the supercritical Reynolds Number range. Examination of the RMS unsteady pressure indicated a sharp peak at the separation point, with a rapid and reasonably symmetric drop in both directions. Figure 1 presents the representation which was developed. Note that the factor

$$
\left[q\left(1-C_{P_{b}}\right)\right]
$$

is just the local free stream dynamic pressure at the separation point. Next a representation of the power

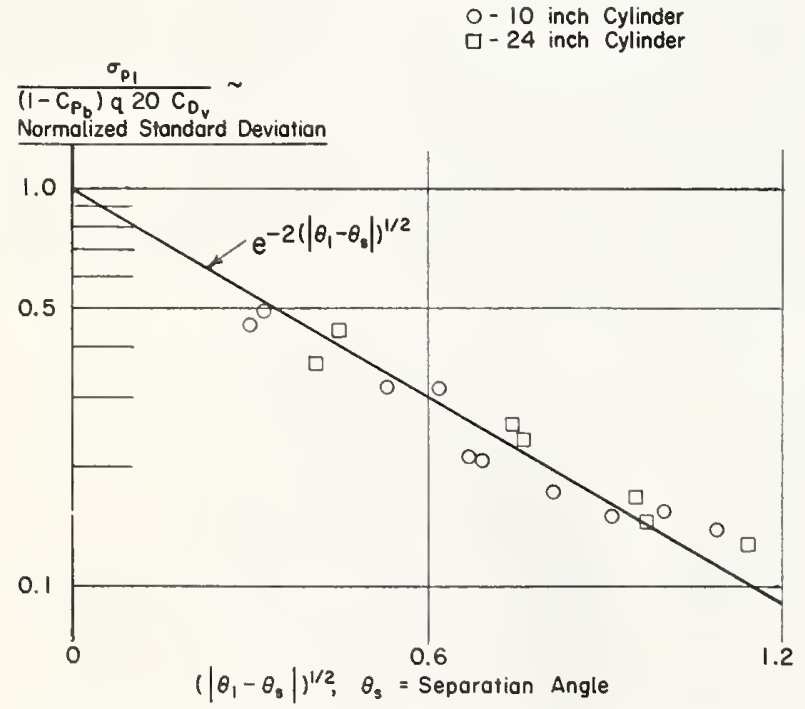

FIgURE 1. Normalized standard deviation of surface pressure on 10-in. and 24-in. cylinders in supercritical flow. spectral density is obtained, as presented in Figure 2. Finally, a representation of cross-power spectral density is obtained as shown in Figure 3. Note that the representation is real. The data analysis showed small imaginary components (phase angle $<10 \mathrm{deg}$ ) with no observable trends. Combining these results, Eq (1) is obtained for the cross-power spectral density of the unsteady pressure in supercritical flow.

$$
\begin{aligned}
F_{p_{1} p_{2}}\left(\omega ; x_{1}, x_{2}, \theta_{1}, \theta_{2}\right)= & \frac{2 D}{U}\left[\left(1-C_{P_{b}}\right) \cdot q \cdot 20 C_{D_{v}}\right]^{2} \\
& \cdot \exp \left\{-2\left[\left(\left|\theta_{1}-\theta_{s}\right|\right)^{1 / 2}\right.\right. \\
& +\left(\left|\theta_{2}-\theta_{s}\right|\right)^{1 / 2}+\left(\frac{\omega D}{U}\right)^{1 / 2} \\
& \left.\left.+\left(\frac{\omega \ell}{U}\right)^{1 / 2}\right]\right\}
\end{aligned}
$$

$\left[F_{P I P 1}(\omega)\right] /\left[\sigma_{P 1}^{2}(2 D / U)\right]$, NORMALIZED

POWER SPECTRAL DENSITY

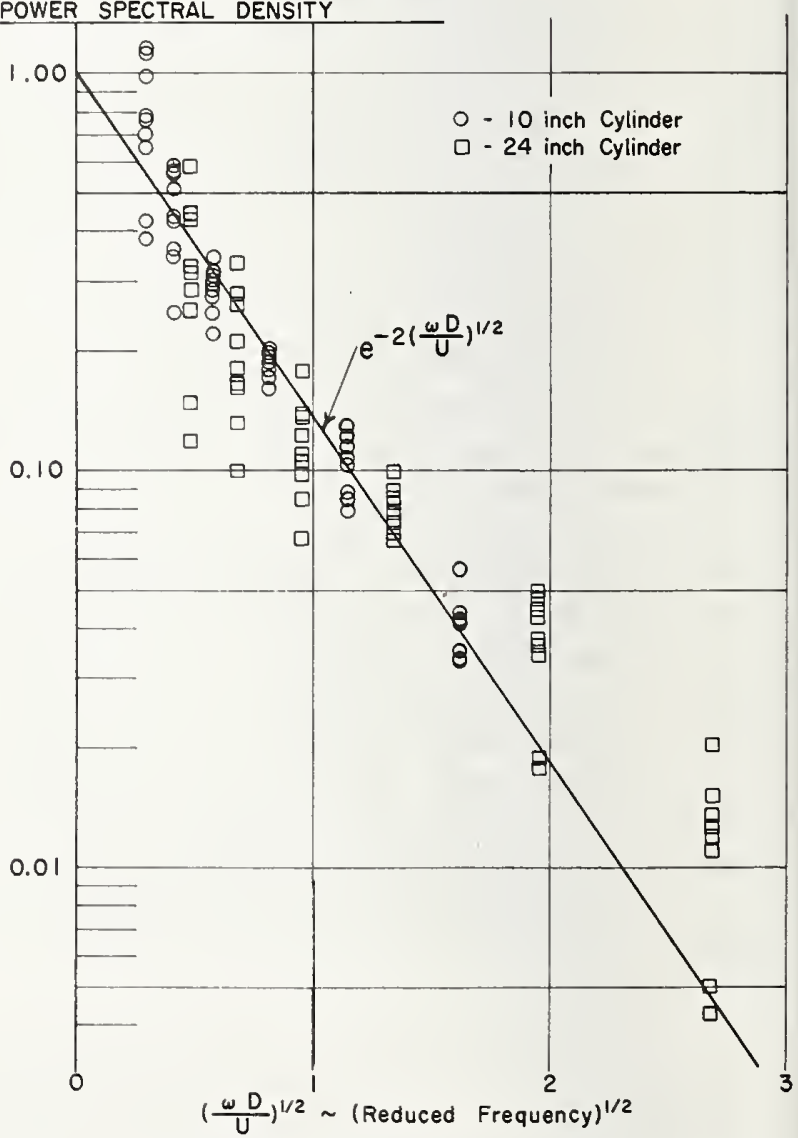

Figure 2. Normalized power spectral density of surface pressure on 10-in. and 24-in. cylinders in supercritical flow. 
10 Rodions $/ \mathrm{sec}=5.45-21.8=43.6-174.5$

24 inch Cylinder - $0-\square=8-\Delta$

$\left[\left|F_{p_{1 P 2}}(\omega)\right|\right] /\left[F_{P_{1 P}}(\omega) F_{p_{2 P 2}}(\omega)\right]^{] / 2}$ Normalized

Cross Power Spectral Density
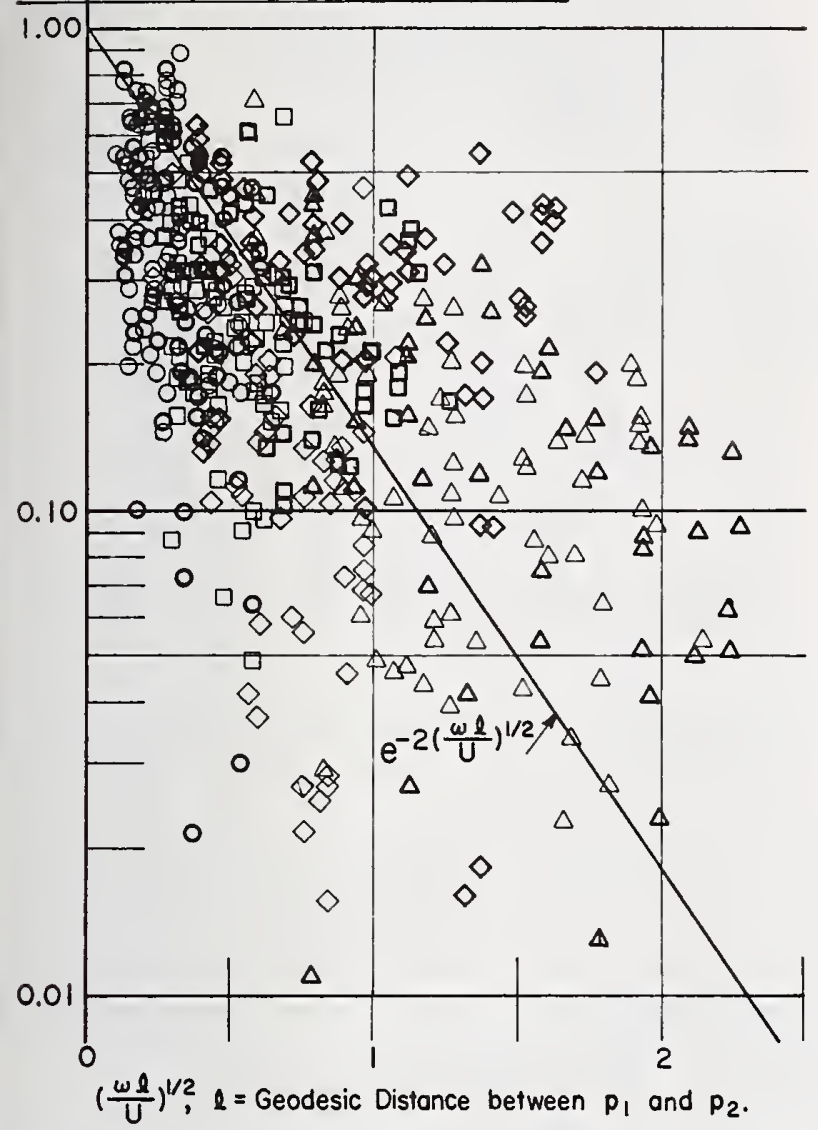

Figure 3. Normalized cross power spectral density of surface pressure on 10-in. and 24-in. cylinders in supercritical flow.

where

$$
\ell=\sqrt{\left(x_{1}-x_{2}\right)^{2}+\left[\frac{\left|\theta_{1}-\theta_{2}\right| \cdot D}{2}\right]^{2}}
$$

Next the data for subcritical and transcritical flow were analyzed. It had originally been intended to consider the regimes separately, but it soon became evident that no qualitative difference could be found between the two regimes. Examining the RMS unsteady pressure (and excluding the region around the separation point where the random unsteady pressure would be significant, it was seen that the important variable was the local dynamic pressure. Figure 4 presents the representation of RMS unsteady pressure which was developed. Figure 5 presents power spectral density computations for the two regimes. It is obvious that the results are essentially identical except for a difference in fre- $\sigma_{p_{1}} /\left(\mathrm{q} 10 \mathrm{C}_{\mathrm{D}_{\mathrm{V}}}\right) \sim$ Normalized

Standard Deviation

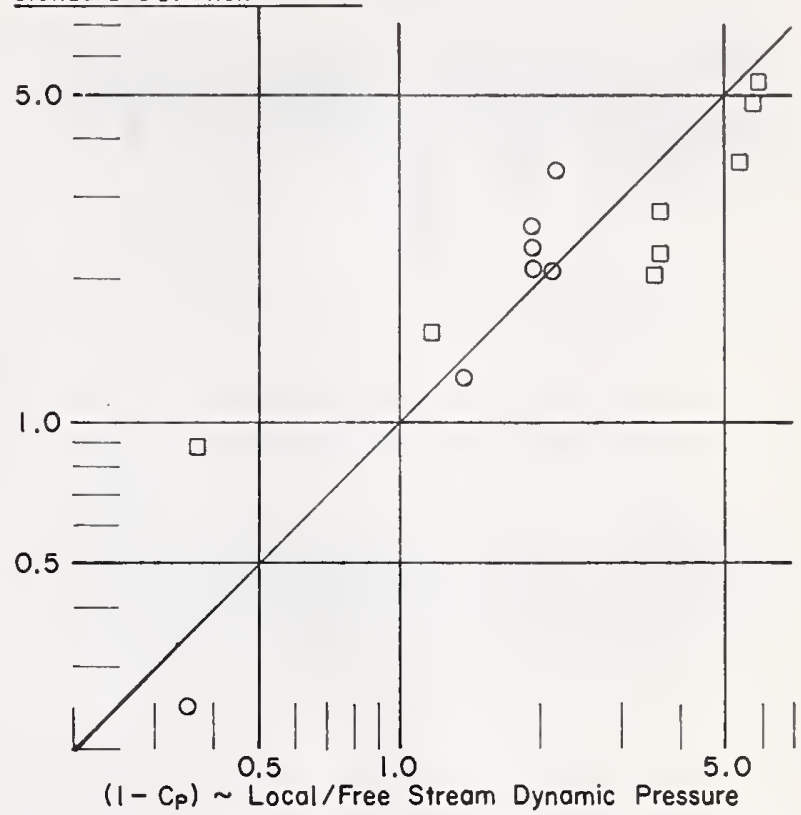

Figure 4. Normalized standard deviation of surface pressure on 10-in. cylinder in subcritical flow and 24-in. cylinder in transcritical flow.

quency, and that the principal component of the signal has a bandwidth less than the analysis bandwidth of either the wideband or the narrow band analysis. The technique developed in Chapter II of Reference 3 is then used to indicate the nature of the signal. Figure 6 presents the results. It is indicated that the signals are a combination of wide band random with a strong periodic component, with no discernible difference between the subcritical and transcritical regimes. It is interesting to note that the oscillator response amplitude distributions show the same characteristics as response of the wind tunnel model. That is, when the oscillator frequency coincides with the Strouhal frequency the amplitude distribution approaches a sinusoidal distribution. Away from the Strouhal frequency, the amplitude distribution approaches a Gaussian distribution. Figure 7 presents plots of surface pressure time history for the two regimes. If allowance is made for the fact that the sampling rate per cycle for transcritical flow is double that for subcritical flow, the two signals have the same appearance. Note that although the signals are periodic, they are not sinusoidal.

Finally, when the cross-power spectral density at the Strouhal frequency is computed, the result is 
extremely simple. All signals on each side of the cylinder are in phase, and one side is just $180 \mathrm{deg}$ out of phase with the other (again within about $\pm 10 \mathrm{deg})$. The correlation is essentially unity $(>0.95)$ for all signals. Combining these results with $\mathrm{Eq}$ (1), Eq (2) is obtained for the unsteady surface pressure on a circular cylinder in two-dimensional flow.

$$
\begin{aligned}
F_{p_{1} p_{2}}\left(\omega ; x_{1}, x_{2}, \theta_{1}, \theta_{2}\right)= & \frac{2 D}{U}\left[\left(1-C_{p_{b}}\right) q \cdot 20 C_{D_{v}}\right]^{2} \\
& \cdot \exp \left\{-2\left[\left(\left|\theta_{1}-\theta_{s}\right|\right)^{1 / 2}\right.\right. \\
& +\left(\left|\theta_{2}-\theta_{s}\right|\right)^{1 / 2}+\left(\frac{\omega D}{U}\right)^{1 / 2} \\
& \left.\left.+\left(\frac{\omega \ell}{U}\right)^{1 / 2}\right]\right\}+\frac{\theta_{1} \theta_{2}}{\left|\theta_{1} \theta_{2}\right|} \\
& \cdot\left[1-C_{P}\left(\theta_{1}\right)\right]\left[1-C_{P}\left(\theta_{2}\right)\right] \\
& \cdot\left[q \cdot 10 C_{D_{v}}\right]^{2} \cdot \delta\left(\delta-\frac{2 \pi \omega D}{U}\right)
\end{aligned}
$$

where

$$
-\pi<\theta<\pi
$$

Note that the range of $\theta$ has set so the cross-power spectral density of the periodic pressure has the proper phase angle. Since $\delta=0$ in the supercritical flow regime, the periodic component vanishes in that regime but is otherwise present. It is interesting to note, in this connection, that satellite pictures of islands in ocean currents show a vortex system in the wake behind the island.

\section{Comparison with Work Under Contract NAS8-11277}

Direct comparisons with the work under Contract NAS8-11277 [4] are difficult since Reference 4 reports measurements of the unsteady aerodynamic force on a segment of a tunnel-spanning cylinder. However, qualitative comparisons can be made. First, Reference 3 presents data indicating that the Strouhal Number increases from 0.15 at $R=2 \times 10^{6}$ to 0.3 at $R=7 \times 10^{6}$ and then is constant at 0.3 at higher Reynolds Number. Reference 1, presenting the original flow field parameter analysis, computes a Strouhal Number for the Saturn V model which
Note: Amplifier Gain Adjusted to Give Approx. 0.2 Volt Signal RMS to Minimize Error in Digitizing

10 inch 24 inch

Cylinder Cylinder

$0-\square$ - Wide Band ( 600 Samples of Signal)

$\triangle----\diamond \quad$-Narrow Band (4000 Samples of Signal)

\section{POWER SPECTRAL DENSITY \\ (Volt) $)^{2}$ - sec/Rodian}

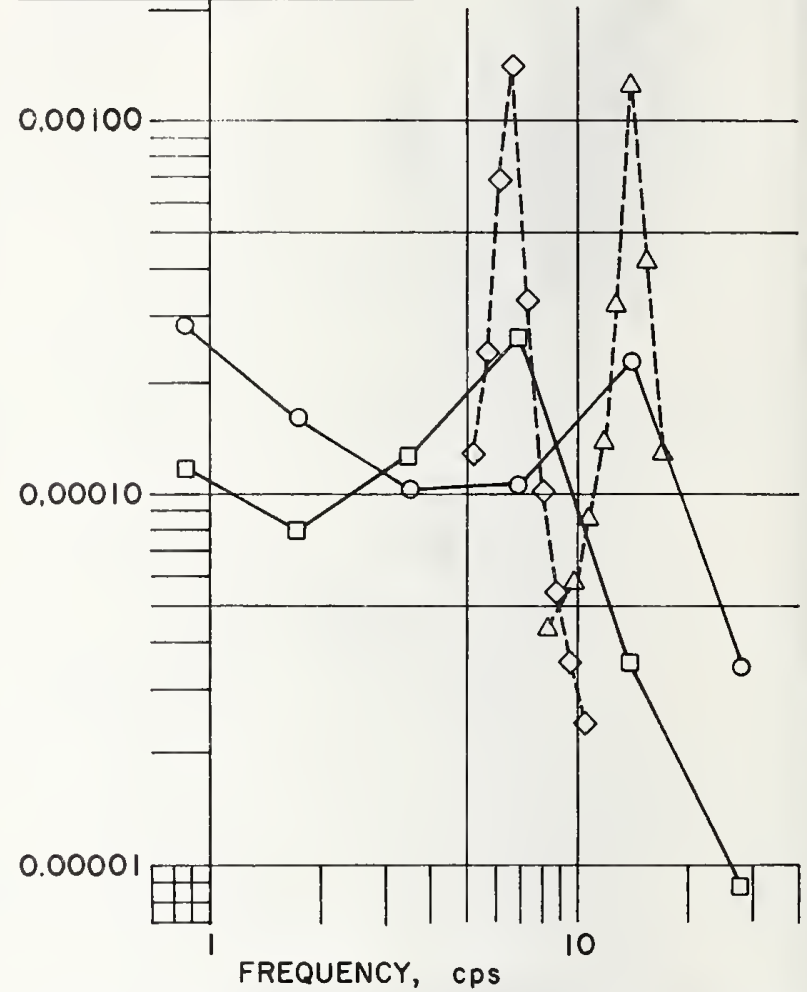

FigURE 5. Effect of bandwidth on computed power spectral density of surface pressure signal for 10-in. cylinder in subcritical flow and 24-in. cylinder in transcritical flow.

is 0.14 at $R=3 \times 10^{6}$, increases to .30 at $R=7 \times 10^{6}$, and is approximately constant at higher Reynolds Numbers.

Second, Reference 4 interprets the observed data to indicate a wide-band random aerodynamic force in the supercritical range, a narrow-band random force in the low transcritical range, and finally a periodic plus random force in the high transcritical range. The flow field parameter analysis, [1], assumed a wide-band random force in the supercritical and a narrow-band random force in the transcritical Reynolds Number range. The present results indicate that the aerodynamic force is wide-band random in the supercritical range, and random plus periodic in both the subcritical and transcritical Reynolds Number regimes. Comparisons with the conclusions 
10 inch Cylinder Acceleration of Wide Band Oscillotor (600 Samples of Signal, $C_{8}=1.01$ )

$\Delta----$ Accelerotion of Norrow Band Oscillator (4000 Somples of Signal, $C_{8}=1.00$ )

24 inch Cylindsr

$\square$ Acceleration of Wide Band Oscillotor (600 Samples of Signol, $C_{8}=0.943$ )

$\checkmark$---- Acceleration of Narrow Band Oscillator

(4000 Samples of Signol, $C_{8}=0.97$ )

$C_{8}$,

NORMALIZED EIGHTH MOMENT COEFFICIENT

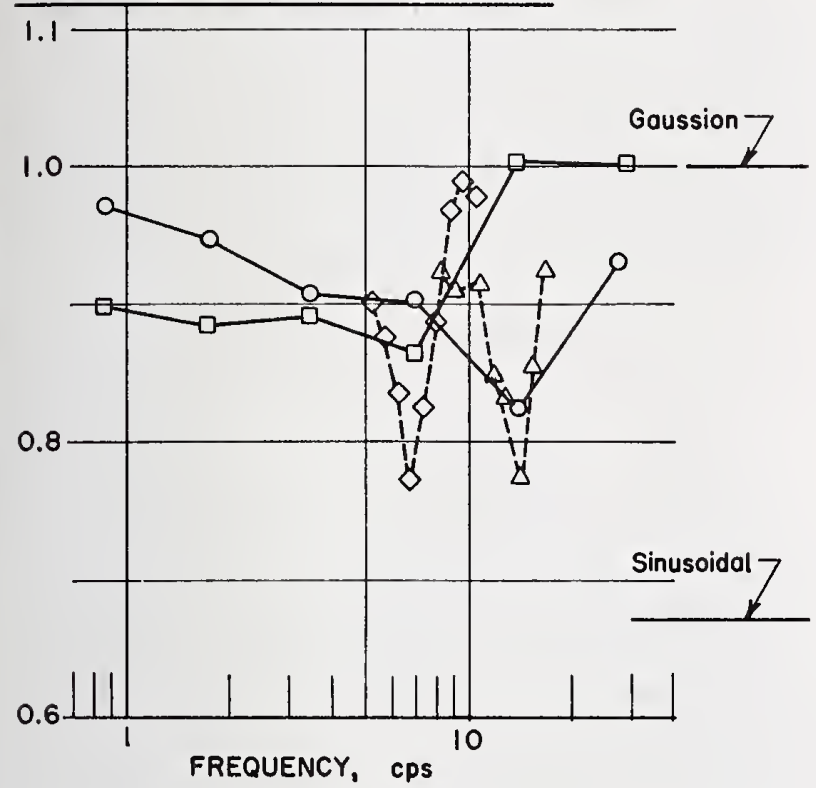

Figure 6. Effect of bandwidth on amplitude distribution of acceleration of oscillator driven by surface pressure signal for 10-in. cylinder in subcritical flow and 24-in. cylinder in transcritical flow.

of Reference 4 concerning the motion dependence of the force and the presence of negative aerodynamic damping cannot be made until measurements of unsteady surface pressure on a moving model can be performed.

\section{Conclusions}

An extremely simple representation of the cross power spectral density of the unsteady surface pressure on a circular cylinder in two dimensional flow has been developed that is a reasonable approximation to measured data. The most important result is
SURFACE PRESSURE SIGNAL, Volis
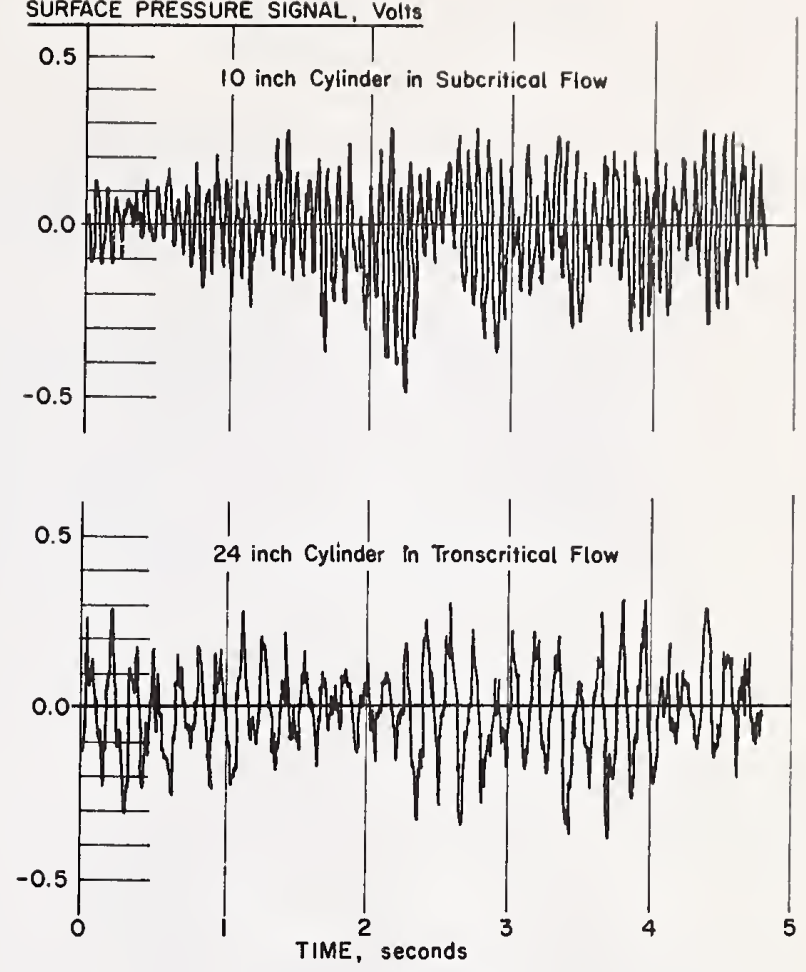

Figure 7. Time history of surface pressure signal for 10-in. cylinder in subcritical flow and 24-in. cylinder in transcritical flow.

the essential similarity between the subcritical and transcritical Reynolds Number regimes.

\section{References}

[1] Petcrson, H. C. and W. E. Simon: Investigations Relative to Wind Induced Oscillations of the Saturn Boosters in the Vertical Launch Position (1 June 1963 thru 30 June 1964), September 1964.

[2] Simon, W. E., C. R. Howard, and H. C. Peterson: Investigations Relative to Wind-Induced Oscillations of Saturn Boosters in the Vertical Launch Position. Annual Report, Contract NAS8-5322, March, 1967.

[3] Simon W. E.: Investigations Relative to Wind-Induced Oscillations of the Saturn Boosters in the Vertical Launch Position. Final Report, Contract NAS8-5322, March, 1968.

[4] Cincotta, J. J.: Experimental Investigation of WindInduced Oscillation Effects on a Circular Cylinder in Two Dimensional Flow. Contract NAS8-11277 (no date). 


\title{
ON THE RELIABILITY OF GUST LOADING FAC'TORS
}

\author{
Barry J. Vickery \\ Faculty of Engineering Science \\ University of Western Ontario \\ London, Ontario, Canada
}

\begin{abstract}
The accuracy of the simplified gust factor approach to the determination of wind loads is examined. An expression for the gust factor is derived which treats the scale of turbulence, mode shape of vibration, and parameters defining the vertical and crosswind cospectra as variables. The method formulated in this paper differs from previous methods primarily in the sizc reduction factor and in the form of the spectrum of velocity fluctuations. Predicted values of gust factors are compared with those observed on eight different aeroelastic models tested in a boundary layer wind tunnel. The gust factor relates only to overall loads in the mean wind direction. Where basic data are well defined, the ratio of expected peak values of wind-induced stress or deflection to their average values can be predicted to an accuracy of typically 5 percent to 10 percent.
\end{abstract}

Key words: Buildings; dynamies; gust factor; mode shape; probability theory; wind loads.

\section{Introduction}

While the dynamic action of wind on tall buildings, towers and other structures has been of some concern to structural engineers for many years, there have been few references to this action in the various building codes. This omission can be explained, in part, by the absence of comparatively simple analyses or rules which would provide a reliable indication of the severity of dynamic action. Attempts to formulate such a set of rules have recently been made by Davenport [1],* Vickery [2], and Vellozzi and Cohen [3]. The aim of all three of these attempts has been the definition of a "gust factor" to be applied to the steady wind forces in order to account for dynamic action.

It is the object of this paper to examine the accuracy of the simplified "gust factor" approach to the assessment of wind loads. In Section 2, an expression for a gust factor is derived. The formulation of the gust factor is similar to that presented by Davenport [1] but an endeavor has been made to reduce the necessary simplifications to a minimum. The parameters involved in the computation of the gust factor are more flexible than those adopted by Davenport and allow the user to take advantage of meteorological data which might be available for a particular site. The scale of turbulence, the parameters defining the vertical and cross-wind cospectra and also the shape of the mode of vibration are

* Figures in brackets indicate literature references at the end of this paper. treated as variables. In previous formulations of a gust factor, these quantities have been assigned specific values.

In Section 3, the validity of the basic assumptions used in the derivation are examined in the light of recent experimental data obtained from both models and full scale structures. The experimental data are employed to obtain a measure of the accuracy of the gust factor approach when used in conjunction with well defined meteorological, aerodynamic, and structural parameters. The loss of accuracy associated with the use of available meteorological data, which may often be poorly defined, is examined in Section 4.

A comparison of gust factors computed by the methods proposed by Davenport, Vickery, and Vellozzi and Cohen with those computed in accordance with this paper is presented in Section 5 .

\section{Derivation of a Gust Factor for a Linear Elastic Structure in Turbulent Shear Flow}

The analysis which follows is based on numerous assumptions and simplifications, these are identified in the analysis and in subsequent sections; the major points will be examined more closely. The basic assumptions on which the analysis is based are as follows:

(a) The load per unit area, projected on a plane normal to the mean wind can be expressed in the form,

$$
q(y, z)=C_{p}(y, z) \rho \frac{u^{2}}{2}(y, z)
$$


in which

$C_{p}(y, z)$ is a pressure coefficient,

$u(y, z)$ is the instantaneous velocity at $(y, z)$ in the absence of the structure.

(b) The response of the structure in the fundamental mode is dominant for both mean and fluctuating deformations.

(c) The intensity of turbulence, $\sqrt{\overline{u^{\prime 2}} / \bar{u}}$, is much less than unity and terms of order $\overline{u^{\prime 2}} / \overline{u^{2}}$ may be neglected.

(d) The wind velocity fluctuations constitute a stationary random process and are distributed according to the normal error law (Gaussian).

The generalized force $Q$ for the fundamental mode of vibration may now be expressed in terms of its mean and time dependent components. If $\mu(y, z)$ is the fundamental mode shape and $\bar{u}(y, z), u^{\prime}(y, z)$ the mean and time dependent components of the "along-wind" velocity, then the mean force may be written as:

$$
\bar{Q}=\int^{A} C_{p}(y, z) \rho \frac{\bar{u}^{2}}{2}(y, z) \mu(y, z) d A
$$

and the time dependent component as:

$Q^{\prime}=\int^{A} C_{p}(y, z) \rho \bar{u}(y, z) u^{\prime}(y, z) \mu(y, z) d A$

The spectral density function $\left(S_{Q}(f)\right)$ of $Q^{\prime}$ may be expressed as:

$$
S_{Q}=\rho^{2} \bar{u}_{o}^{2} \iint S_{u}\left(y_{1} z_{1} y_{2} z_{2} f\right) \psi\left(y_{1} z_{1}\right) \psi\left(y_{2} z_{2}\right) d A_{1} d A_{2}
$$

in which

$$
\psi(y, z)=C_{p}(y, z) \mu(y, z) \frac{\bar{u}(y, z)}{\bar{u}_{o}}
$$

where $\bar{u}_{o}$ is a reference velocity and $S_{u}\left(y_{1} z_{1} y_{2} z_{2} f\right)$ is the cross-spectral density function of the fluctuating along-wind components of velocity at $\left(y_{1}, z_{1}\right)$ and $\left(y_{2}, z_{2}\right)$.

In order to reduce the expression for $S_{Q}(f)$ to a form suitable for the derivation of a gust factor, certain simplifications and restrictions are necessary. The simplifications to be made at this stage are as follows:

(e) The structure is free-standing with a fundamental mode shape that is adequately approximated by power law variation in displacement from zero at the base to a maximum at the tip, i.e. $\mu(y, z)=\left(\frac{z}{h}\right)^{\beta}$,

(j) The projected area of the structure is rectangular in shape with a height $h$ and breadth $b$.

(g) The mean velocity is a function of $z$ only and can be expressed in the form,

$$
\frac{\bar{u}(z)}{\bar{u}(h)}=\left(\frac{z}{h}\right)^{\alpha}
$$

(h) The cross - spectral density function, $S_{u}\left(y_{1} z_{1} y_{2} z_{2} f\right)$, can be expressed in the form:

$$
\left(y_{1} z_{1} y_{2} z_{2} f\right)=S_{u}(f) \cdot R\left(y_{1} y_{2} z_{1} z_{2} f\right)
$$

in which,

$S_{u}(f)$ is the spectral density function of $u^{\prime}$ and is invariant with $y$ and $z$,

(i) The function $R\left(y_{1} y_{2} z_{1} z_{2} f\right)$ can be expressed in the form:

$$
R=\exp \frac{-f \cdot \sqrt{C_{y}{ }^{2}\left(y_{1}-y_{2}\right)^{2}+C_{z}{ }^{2}\left(z_{1}-z_{2}\right)^{2}}}{1 / 2\left(\bar{u}\left(z_{1}\right)+\bar{u}\left(z_{2}\right)\right)}
$$

in which $C_{y}$ and $C_{z}$ are constants, invariant with $y$ and $z$.

( $j) C_{p}(y, z)$ is constant over the structure and equal to $C_{D}$ where:

$$
C_{D}=\frac{\bar{F}}{\int 1 / 2 \rho \bar{u}^{2}(z) d A}
$$

The spectral density function $S_{Q}(f)$ may then be expressed as follows:

$$
S_{Q}(f)=\left\{\frac{C_{D} \rho \bar{u}^{2}(h) b h}{2(1+\beta+\alpha)}\right\}^{2} \frac{4 \overline{u^{\prime 2}}}{\overline{u^{2}(h)}} \cdot S_{u}{ }^{\prime}(f) \cdot \chi^{2}(F, \lambda)
$$

in which,

$$
\begin{aligned}
& S_{u}{ }^{\prime}(f)=\frac{1}{\overline{u^{\prime 2}}} S_{u}(f) \\
& \chi^{2}(F, \lambda)=(1+\beta+\alpha)^{2} \int^{1} \int^{1} \int^{1} \int^{1} z_{1}^{\beta+\alpha} z_{2}{ }^{\beta+\alpha} \\
& \cdot \exp \left(\frac{-F \sqrt{\left(z_{1}-z_{2}\right)^{2}+\lambda^{2}\left(y_{1}-y_{2}\right)^{2}}}{1 / 2\left(z_{1}^{\alpha}+z_{2}{ }^{\alpha}\right)}\right) \\
& d y_{1} d y_{2} d z_{1} d z_{2}
\end{aligned}
$$

and

$$
\lambda=\frac{C_{y} b}{C_{z} h}, \quad F=\frac{C_{z} f H}{\bar{u}(H)}
$$


The function $\chi^{2}(F, \lambda)$ is also a function of the power-law exponents $\alpha, \beta$ but the variation with $\alpha$ and $\beta$ about average values of these quantities is small and may be ignored. The function has been computed by numerical integration and is presented in Figure 1. For the limiting cases of $F \rightarrow 0$ and $F \gg 1$ the function can be evaluated analytically:

$$
\begin{aligned}
& \chi^{2}(F \rightarrow 0, \lambda) \rightarrow 1 \\
& \chi^{2}(F \gg 1, \lambda) \rightarrow \frac{2 \pi}{\lambda} \cdot \frac{1}{F^{2}}\left\{\frac{(1+\beta+\alpha)^{2}}{1+2 \beta+4 \alpha}\right\}
\end{aligned}
$$

The mean deflection of the structure $\bar{a} \mu(z)$ and the root-mean-square deflection $\sqrt{\overline{a^{\prime 2}}} \cdot \mu(Z)$ may be expressed as follows:

$$
\begin{aligned}
& \bar{a}= \frac{1}{1+\beta+\alpha}\left\{C_{D} \rho \frac{\bar{u}^{2}(H)}{2} b h\right\} /\left(2 \pi f_{o}\right)^{2} \cdot M(11) \\
& \sqrt{\overline{a^{\prime 2}}}=\frac{1}{(1+\beta+\alpha)}\left\{C_{D} \rho \frac{\bar{u}^{2}(H)}{2} b h\right\} \frac{2 \cdot \overline{u^{\prime 2}}}{\bar{u}(H)} \\
& \cdot\left\{\int_{0}^{\infty} S_{u}{ }^{\prime}(f)|H(f)|^{2} \chi^{2}(F, \lambda) d f\right\}^{1 / 2} \\
& \frac{\left(2 \pi f_{o}\right)^{2} \cdot M}{}
\end{aligned}
$$

in which,

$$
M=\int_{0}^{H} m(z) \mu^{2}(z) d z
$$

$m(z)=$ mass per unit height

$$
f_{o}=\text { natural frequency of vibration }
$$

$$
|H(f)|^{2}=\frac{1}{\left(1-\left(\frac{f}{f_{o}}\right)^{2}\right)^{2}+4 \eta^{2}\left(\frac{f}{f_{o}}\right)^{2}}
$$

$\eta=$ damping ratio (fraction of critical).

The ratio of the RMS value to the mean value (the coefficient of variation) of the response is then,

$$
\begin{aligned}
\frac{\sqrt{\overline{a^{\prime 2}}}}{\bar{a}}= & \frac{1+\beta+2 \alpha}{1+\beta+\alpha} \cdot \frac{2 \sqrt{\overline{u^{\prime 2}}}}{\bar{u}(h)} \\
& \cdot\left\{\int_{0}^{\infty} S_{u}{ }^{\prime}(f) \cdot|H(f)|^{2} \cdot \chi^{2}(F, \lambda) d f\right\}^{1 / 2}
\end{aligned}
$$

The integral with respect to frequency can conveniently be approximated by the following expression:

$$
\begin{aligned}
\int_{0}^{\infty} S_{u}{ }^{\prime}(f) \cdot|H(f)|^{2} \chi^{2}(F, \lambda) d f \\
=\int_{0}^{\infty} S_{u}{ }^{\prime}(f) \chi^{2}(F, \lambda) d f \\
+S_{u}{ }^{\prime}\left(f_{o}\right) \frac{\pi f_{o}}{4 \eta} \chi^{2}\left(F_{o}, \lambda\right)
\end{aligned}
$$

in which,

$$
\begin{aligned}
f_{o} & =\text { natural frequency, } \\
F_{o} & =C_{z} f_{o} h / \bar{u}(h) .
\end{aligned}
$$

The form of $S_{u}{ }^{\prime}(f)$ after Davenport [4] is:

$$
S_{u}{ }^{\prime}(f)=\frac{\frac{2}{3} \cdot \frac{f L(h)}{\bar{u}(h)} \cdot \frac{L(h)}{\bar{u}(\mathrm{~h})}}{\left\{1+\left(\frac{f \cdot L(h)}{\bar{u}(h)}\right)^{2}\right\}^{4 / 3}}
$$

in which $L(h)$ is a length scale and is equal to $\sqrt{3}$ times the wavelength which the function $f \cdot S_{u}{ }^{\prime}(f)$ is a maximum. Davenport originally suggested that

$$
L(h)=4000 \frac{\bar{u}(h)}{\bar{u}(33)},
$$

however, there are advantages in retaining the more flexible form of Equation 15. If a reduced frequency

$$
n=\frac{f L(h)}{\bar{u}(h)}
$$

is introduced, then Equation 15 may be re-written as follows:

$$
\begin{aligned}
\frac{\sqrt{\overline{a^{\prime 2}}}}{\bar{a}}= & \left\{\frac{1+\beta+2 \alpha}{1+\beta+\alpha} \frac{2 \sqrt{\overline{u^{\prime 2}}}}{\bar{u}(h)}\right\}\left\{\frac{2}{3} \int_{0}^{\infty} \frac{n}{\left(1+n^{2}\right)^{4 / 3}} \chi^{2}\right. \\
& \cdot\left(F=\frac{n C_{z} h}{L(h)}, \lambda\right) d n+\frac{\pi}{6} \cdot \frac{n_{o}^{2}}{\left(1+n_{o}^{2}\right)^{4 / 3}} \\
& \left.\cdot \frac{1}{\eta} \cdot \chi^{2}\left(F_{o}, \lambda\right)\right\}^{1 / 2} \\
= & r\left\{B+\frac{S \cdot F}{\eta}\right\}^{1 / 2}
\end{aligned}
$$

where

$$
r=\frac{1+\beta+2 \alpha}{1+\beta+\alpha} \cdot \frac{2 \overline{u^{\prime 2}}}{\bar{u}(h)} \quad \text { (roughness factor) }
$$


$=$ Function terrain roughness, building height mode shape

$S=\chi^{2}\left(F_{o}, \lambda\right) \quad$ \{size reduction factor

= Function $\left\{\frac{C_{z} f_{o} h}{\bar{u}(h)}, \frac{C_{z} h}{C_{z} h}\right\}$

$F=\frac{\pi}{6} \cdot \frac{n_{o}{ }^{2}}{\left(1+n_{o}^{2}\right)^{4 / 3}} \quad$ \{gust energy factor

= Function $\left\{\frac{f_{o} L(h)}{\bar{u}(h)}\right\}$

$B=$ Function $\left\{\frac{C_{Z} h}{L(h)}, \frac{C_{y} B}{C_{z} H}\right\} \quad \begin{aligned} & \text { (Background } \\ & \text { excitation factor) }\end{aligned}$

The parameters $S, B, F$ and $r$ have been computed and are presented in Figures 1 to 4 .

A gust factor $G$ is defined as the ratio of the expected peak value of displacement in a period $T$ to

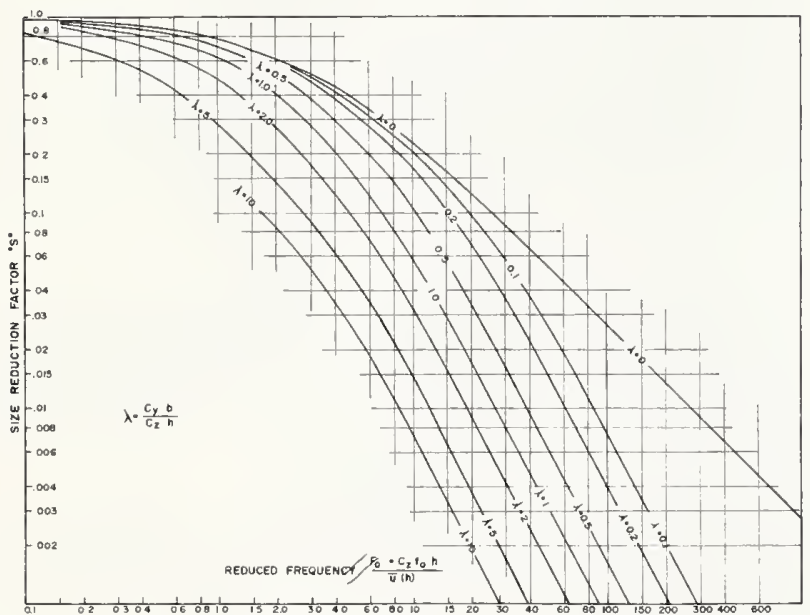

Figure 1. Size reduction factor "S."

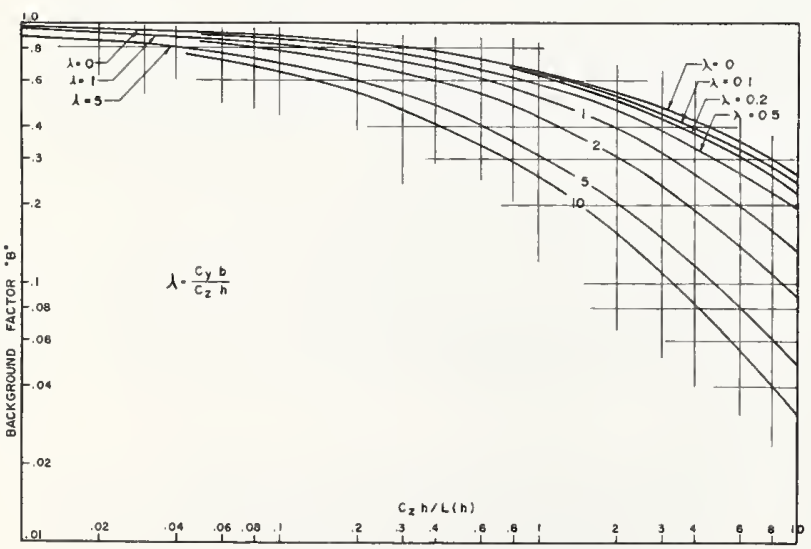

Figure 2. Backround factor " $B$."

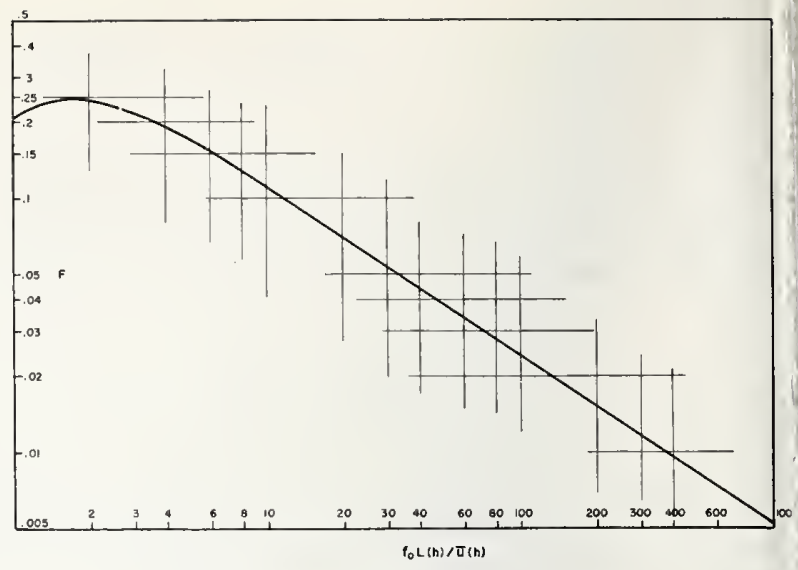

Figure 3. Gust energy factor " $F$."

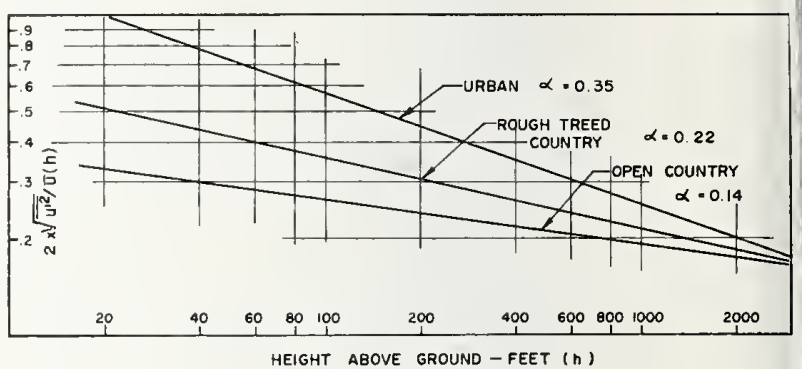

FIGURE 4. Suggested values of $\frac{2 \sqrt{\overline{u^{\prime 2}}}}{\bar{u}(h)}\left(=r \cdot \frac{1+\beta+\alpha}{1+\beta+2 \alpha}\right)$.

the mean value. The gust factor may now be written as:

$$
G=1+\bar{g} r\left\{B+\frac{S F}{\eta}\right\}^{1 / 2}
$$

in which $\bar{g}$ is a "peak factor" and is equal to the ratio of the expected maximum value of the dynamic displacement to the root-mean-square value of $\mathrm{dy}$ namic displacement. Davenport [5] has derived the relationship:

$$
\bar{g}=\sqrt{2 \log _{e} \nu T}+\frac{0.577}{\sqrt{2 \log _{e} \nu T}}
$$

$\bar{g}$ is the average or expected value (over a large number of periods each of duration $T$ ) of $g$, the ratio of the maximum to root-mean-square values in each period of duration $T$. The standard deviation of $g$ has also been derived by Davenport and is:

$$
\sigma_{g}=\frac{\pi / \sqrt{6}}{\sqrt{2 \log _{e} \nu T}}
$$

In Equations $1 S$ and $19, \nu$ is the equivalent frequency and is given by the relationship: 


$$
v^{2}=\frac{\int_{0}^{\infty} S(f) \cdot f^{2} d f}{\int_{0}^{\infty} S(f) d f}
$$

$S(f)=$ spectral density function of the dynamic response.

Equations (18) and (19) are valid only for large values of $\nu T$, for lightly damped low frequeney structures the value of $\nu T$ is not sufficiently great (for $T \simeq 20$ min. say) and the use of Equations (18) and (19) will result in an overestimate of $\bar{g}$ and underestimate $\sigma_{\theta}$.

In the work which follows, it will be assumed that $\bar{g}$ is equal to 3.5 . The value of 3.5 is, on average, slightly conservative; for values of $T$ between 10 and 30 minutes and for a wide range of structures $\bar{g}$ will usually lie between 3.0 and 3.7 .

\section{Experimental Confirmation of Gust Factor Computations}

Basic to the derivation of a gust factor is the assumed relationship between pressure and velocity. In Section 2, it is taken that the force per unit projected area is proportional to momentum flux (in the absence of the structure). In detail this assumption is far from adequate, however when the concern is with total effects (e.g. total force on a building) many of the inadequacies in detail vanish during the integration. Pressure measurements on both model $[6,7]$ and full scale $[8]$ buildings in turbulent shear flow indicate that both mean and fluctuating pressures on the windward face do reflect the variations in momentum flux. This is particularly true away from the edges of the face; near both the side and the top edges there is a reduction in both the mean and the fluctuating components of pressure. Measurements of the spectrum of the pressure fluctuations on the windward face have shown [8], on both model and full scale structures, the same form as that observed for the velocity spectrum. In general, the pressures on the leeward face, and on side faces, do not reflect the variations in momentum flux but are more or less uniform. Although this latter feature is a major departure from the basic assumption, these wake pressures do not contribute markedly to the total force on a building. Field observations $[8,9]$ on buildings in a city environment indicate that the load on the leeward face is less than $10 \%$ to $20 \%$ of the total load. This proportion would undoubtedly be higher for tower-like structures with $b / h$ ratios substantially less than the values of 0.4 and 1.6 applicable to the structures examined in references [8] and [9]. The wake pressures for very stender structures will, however, reflect the bulk of the turbulent velocity fluctuations; this has been observed in model studies of a very slender tapered stack but the results are as yet unpublished.

\subsection{Expcrimental Determinations of the Size Reduction Factor, $S$}

The adequacy of the pressure/velocity assumption, in so far as it influences the computation of a gust factor, is best examined by a comparison of experimental and predicted values of the size reduction factor $S$. Measurements of this quantity, together with the predicted values, are presented in Figure 5. Three sets of measurements are presented; the most complete of these is that obtained by wind tunnel studies and described elsewhere [10] in some detail. This set of measurements was obtained from plates and prisms of various aspect ratios, the hatched area in Figure 5 indicates the spread in the observations. The remaining two sets of data are from measurements in the atmosphere and are described in references [11] and [12]. The set described in reference [12] was obtained from observations of the fluctuating loads on three mesh targets with areas from $20 \mathrm{ft}^{2}$ to $60 \mathrm{ft}^{2}$ and mounted about $40 \mathrm{ft}$ above ground. The remaining results were obtained from measurements on a building in an urban environment; unfortunately, the atmospheric conditions existing for this set were poorly defined and the results must be regarded with some degree of suspicion.

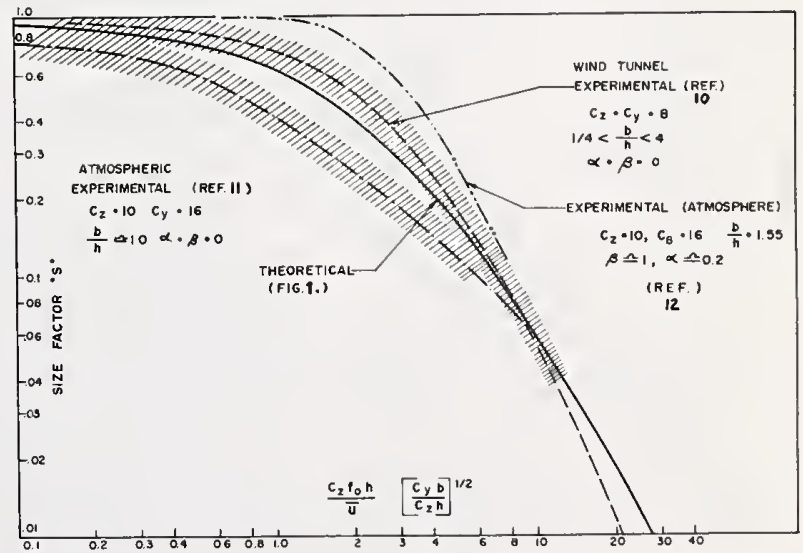

Figure 5. Comparison of experimental and theoretical values of the size factor " $S$. " 
The predicted values of $S$ are in reasonable agreement with the wind tunnel observations, the atmospheric results show similar trends but are limited and, unfortunately, of doubtful value. The most significant values of $S$ are those corresponding to excitation at the natural frequency of vibration. For most structures such excitation will correspond to values of

$$
\frac{C_{Z} f_{o} L}{\bar{u}}\left(\frac{C_{y} b}{C_{Z} h}\right)^{1 / 2}
$$

in the vicinity of $10 \pm 5$. Within this range it would appear that the predicted values of $S$ will be within $\pm 30 \%$ of the true value. While this may appear to be an excessive discrepancy the error induced in a predicted gust factor would generally be from $5 \%$ to $10 \%$.

\subsection{Experimental Determinations of the Parameter $B$}

The background excitation factor $B$ is largely dependent of $S$ and, hence, on the assumed load/ velocity relationship. Predicted and measured values of $\sqrt{\bar{B}}$ are presented in Figure 6 ; the experimental measurements are described in detail elsewhere $[10,13]$. The results were obtained in wind tunnels

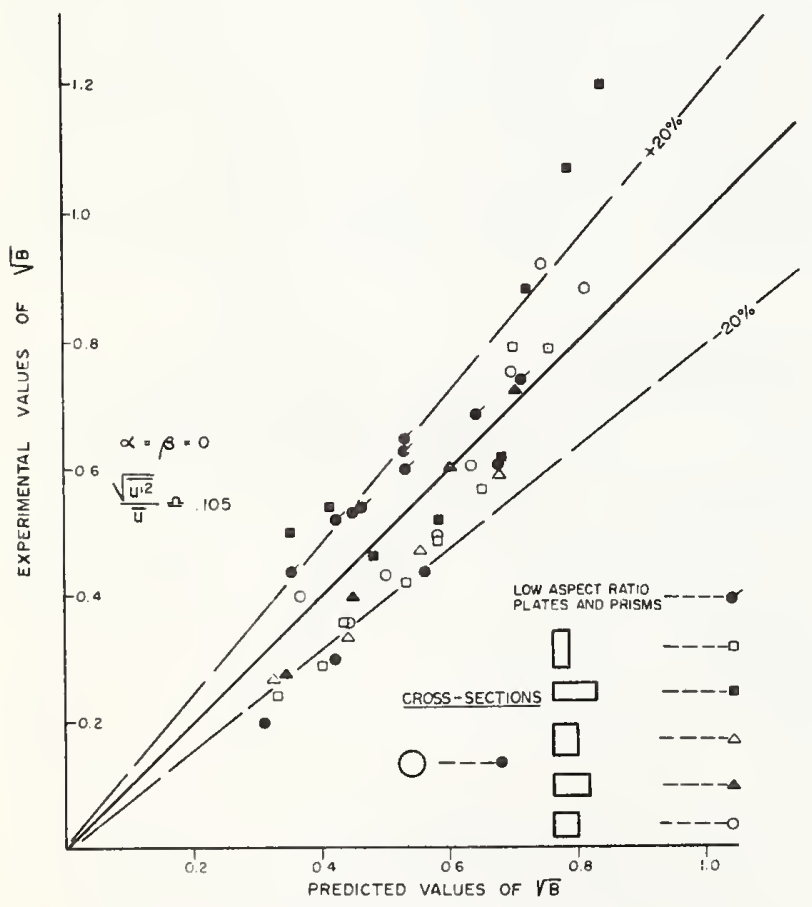

Figure 6. Comparison of observed and predicted values of $\sqrt{\bar{B}}$. and on a wide range of models of different crosssectional shapes and aspect ratios. In all, 45 models were tested and the average value of the ratio

$$
\frac{\sqrt{B_{\text {predicted }}}}{\sqrt{B_{\text {obs. }}}}
$$

was $0.9 \mathrm{~S}$ and the standard deviation of this ratio, 0.18 . This deviation would result in errors of typically $5 \%$ to $10 \%$ in predicted gust factors.

\subsection{Experimental Measurements of Gust Factor}

A direct measure of the reliability of gust factors can be obtained from a comparison of predicted values with those observed on model structures tested in a boundary layer wind tunnel. The shapes of eight aeroelastic models that have been studied in the Boundary Layer Wind Tunnel Laboratory of The University of Western Ontario are shown in Figure 7. The experimental observations for models I to VI are reported in reference [6] and the observations for models VII and VIII in reference [7] and reference [14] respectively. The structure of the flow in the boundary layer wind tunnel is also described in these three reports and is summarized in Table I.

The predicted and observed gust factors for Models I to VI are presented in Table II. Also included in this table are observed values of a lateral gust factor, this factor is the ratio of the peak lateral response in direction $X X$, (i.e. with wind along $Y Y$ ) to the mean drag response in direction $X X$. There are no predicted values of lateral response since, at

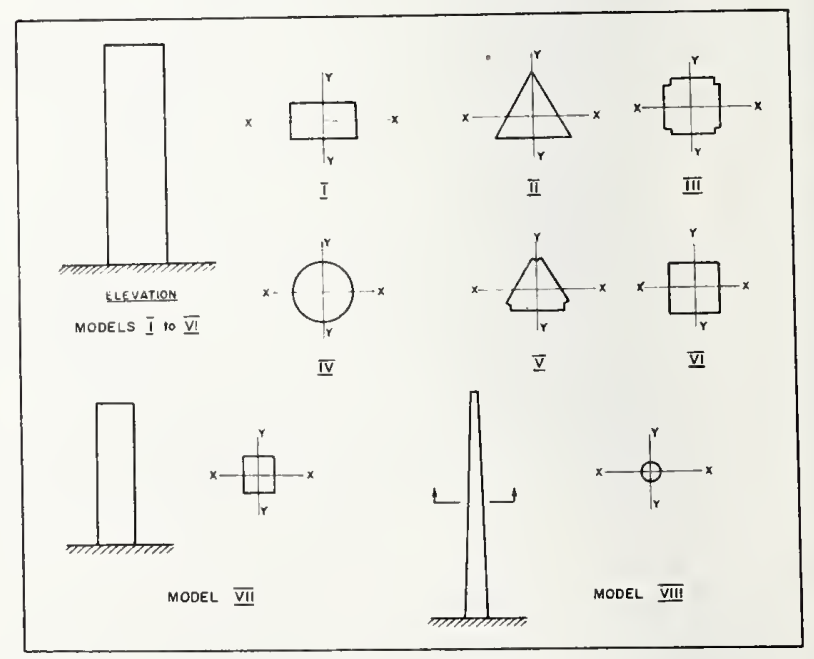

FIGURE 7. Llevations and cross-sections of models studied in B.I.W.T. 
TABLE I. Properties of turbulent shear flow in boundary layer wind tunnel

\begin{tabular}{|c|c|c|}
\hline & $\begin{array}{l}\text { Urban } \\
\text { exposure }\end{array}$ & $\begin{array}{c}\text { Open } \\
\text { exposure }\end{array}$ \\
\hline $\begin{array}{l}\text { Approximate height of rough- } \\
\text { ness elements. } \\
\text { Thickness of boundary layer } \\
\text { Mean velocity profile } \\
\quad \text { (exponent } \alpha \text { ) } \\
\text { Turbulent intensity at } z=2.0 \mathrm{ft} \\
L(z) \text { at } 2=2.0 \text { feet } \\
C_{z}\end{array}$ & $\begin{aligned} & 0.25 \mathrm{ft} . \\
& 4 \mathrm{ft} . \\
& \\
& 0.36 \\
& 0.14 \\
& 14 \mathrm{ft} . \\
& \simeq 10 \\
& \simeq 12\end{aligned}$ & $\begin{aligned} & 0.01 \mathrm{ft} . \\
& 2.5 \mathrm{ft} . \\
& \\
& 0.15 \\
& 0.10 \\
& 10 \mathrm{ft} . \\
& \simeq 10 \\
& \simeq 12\end{aligned}$ \\
\hline
\end{tabular}

present, no adequate theory exists. It will be noted that in six of the eighteen tests, the lateral response exceeds the predicted drag response and in each instance the reduced velocity was the higher of the two velocities examined. The two velocities correspond to approximately $65 \mathrm{mph}$ and $120 \mathrm{mph}$ at prototype scale. Neglecting the lateral response, the observed and predicted gust factors are in satisfactory agreement. The ratio of the predicted to the observed values has an average of 1.04 and a standard deviation of 0.05 .

The predicted and observed gust factors for Model VII are presented in figures 8 and 9 . All results refer to the response along the $Y Y$ axis or normal to the broader face of the model, gust factors were obtained for both urban and open exposures. In Figure 8 the variation of gust factor with total damping is presented while in Figure 9, the gust factor is shown as a function of wind speed. The agreement between the predicted and the observed gust factors is satisfactory and the dependence of the gust factor on both wind speed and damping is clearly demonstrated. The lateral gust factor is the more sensitive to both damping and wind speed.

The gust factors for Model VIII, a tapered stack of circular cross-section, are shown in Figure 10. In this case two predicted values of gust factor are shown, one of these is that derived from the gust factor equation and the second is the result of a more complete analysis which accounts for the true mode shapes and for contributions from modes other than the fundamental. The two predicted values differ only slightly and both are in satisfactory agreement with the observed values.

The results presented in Figures 8, 9, 10 and in Table II cover, in all, 34 evaluations of the gust factor. The range of variables is wide and includes a variety of cross-sections, aspect ratios from 3 to 20 , two conditions of exposure and values of structural damping from 0.001 to 0.02 . The average ratio of the predicted to the observed gust factor is 1.02 and the standard deviation 0.07 . The standard

TABLE II. Measured and predicted gust factors for models I to VI (urban exposure)

\begin{tabular}{|c|c|c|c|c|c|c|}
\hline Model & Line of response & $\bar{u}(h) / n \sqrt{A}$ & $G_{\mathrm{PRED}}$ & $G_{\mathrm{OBg}}$ & $\frac{G_{\mathrm{PRED}}}{G_{\mathrm{OBS}}}$ & $G_{\text {LATERAL }}$ \\
\hline \multirow[t]{4}{*}{ I } & $Y Y$ & 7.0 & 2.48 & 2.55 & 0.97 & 1.53 \\
\hline & & 3.8 & 2.17 & 2.39 & 0.91 & 1.14 \\
\hline & $X X$ & 7.0 & 3.02 & 2.62 & 1.15 & $* 4.94$ \\
\hline & & 3.8 & 2.45 & 2.35 & 1.04 & 2.36 \\
\hline \multirow[t]{4}{*}{ II } & $Y Y$ & 7.0 & 2.44 & 2.40 & 1.02 & 1.99 \\
\hline & & 3.8 & 2.13 & 2.03 & 1.05 & 1.65 \\
\hline & $X X$ & 7.0 & 2.61 & 2.33 & 1.12 & 1.58 \\
\hline & & 3.8 & 2.21 & 2.12 & 1.04 & 0.82 \\
\hline \multirow[t]{2}{*}{ III } & $X X \& Y Y$ & 7.0 & 2.83 & 2.63 & 1.08 & ${ }^{*} 3.64$ \\
\hline & & 3.8 & 2.33 & 2.12 & 1.10 & 1.52 \\
\hline \multirow[t]{2}{*}{ IV } & $X X \& Y Y$ & 7.0 & 2.75 & 2.64 & 1.04 & $* 4.47$ \\
\hline & & 3.8 & 2.29 & 2.29 & 1.00 & 1.71 \\
\hline \multirow{4}{*}{$\mathrm{V}$} & $Y Y$ & 7.0 & 2.65 & 2.64 & 1.00 & ${ }^{*} 3.08$ \\
\hline & & 3.8 & 2.24 & 2.08 & 1.08 & 2.00 \\
\hline & $X X$ & 7.0 & 2.73 & 2.73 & 1.00 & *3.32 \\
\hline & & 3.8 & 2.30 & 2.24 & 1.03 & 1.38 \\
\hline \multirow[t]{3}{*}{ VI } & $X X \& Y Y$ & 7.0 & 2.77 & 2.77 & 1.00 & ${ }^{*} 3.93$ \\
\hline & & 3.8 & 2.28 & 2.18 & 1.05 & 1.57 \\
\hline & & \multicolumn{5}{|c|}{ Average: $1.04 \pm .05$} \\
\hline
\end{tabular}



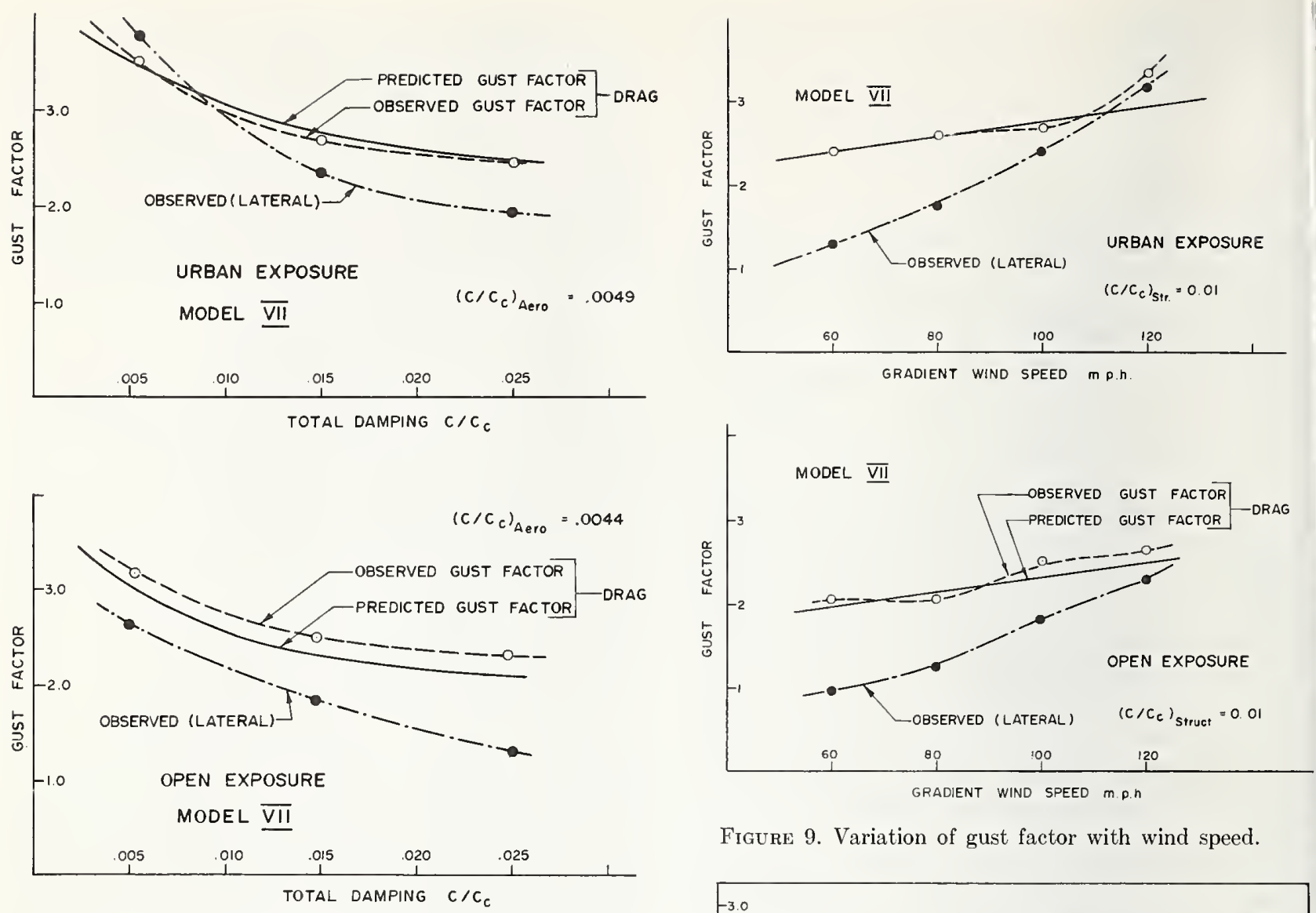

Figure 8. Variation of gust factor with damping.

deviation is in agreement with that deduced from the earlier comparisons of experimental and predicted values of the parameters $S$ and $B$.

\section{The Reliability of Gust Factor Estimates in Relation to The Adequacy of Meteorological and Other Data}

The reliability or accuracy of an estimate of a gust factor is dependent firstly upon the adequacy of the theory and the basic assumptions on which this is founded and, secondly, the accuracy or reliability of the data (meteorological, aerodynamic and structural). The adequacy of the theory has been examined in the previous section; under controlled test conditions with reliable data the theory leads to estimates of the gust factor which are generally within $10 \%$ of observed values. The use of the gust factor to predict the response of a proposed structure to atmospheric winds involves data which, in many instances, is poorly defined. It is intended to determine the relationship between the accuracy of an estimate of a gust factor and the accuracy of the

FIGURE 9. Variation of gust factor with wind speed.

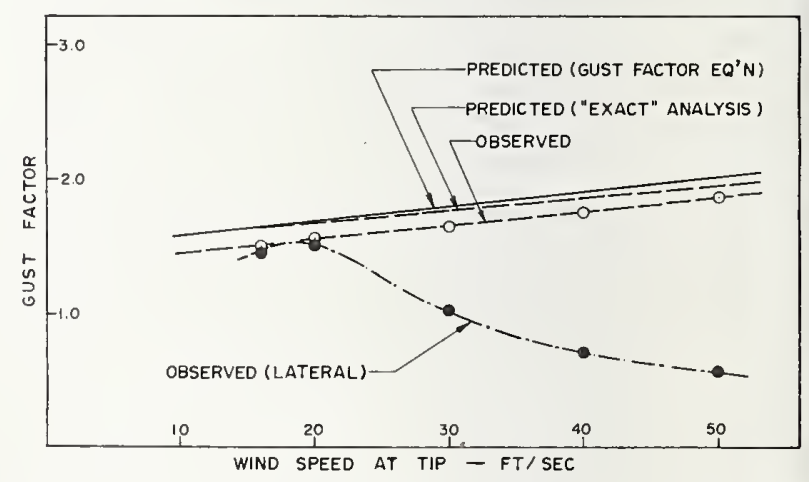

Figure 10. Observed and predicted gust factors for a model tapered stack of circular cross-section.

data, or the independent variables of which it is a function. In the derivation of a gust factor presented in Section 2, it is shown that the gust factor can be expressed as follows:

$$
G=\Phi\left(\alpha, i, L(h), \nu, C_{Z}, C_{y} / C_{Z}, \beta, f_{o}, \eta\right)
$$

in which

$\alpha=$ power law exponent of mean velocity profile,

$i=$ intensity of turbulence,

$L(h)=$ a length proportional to the scale of turbulence, 
$\nu=a$ coefficient representing the variation in the dimensionless spectral density function at a given frequency about some assumed value of the spectral density. i.e., $S\left(f_{o}\right)=\nu \cdot S_{a}\left(f_{o}\right)$ where $S\left(f_{o}\right)$ is the true spectral density function at a given site, and $S_{a}\left(f_{o}\right)$ is the assumed value determined in accordance with, say, the suggestions of Davenport.

$C_{z}=$ a coefficient (defined in Section 2) related to the vertical scale of a turbulence component of a given wavelength.

$C_{y} / C_{z}=$ the ratio of the horizontal to the vertical scale coefficient.

$\beta=$ the power law exponent of a curve approximating to the mode shape of the fundamental mode of vibration.

$f_{o}=$ the natural frequency of vibration of the fundamental mode.

$\eta=$ the total damping, expressed as a fraction of critical.

None of the nine independent variables can be defined with complete confidence. It is difficult to assign an accuracy with which any one variable can be estimated for a given site and structure, but from a survey of existing data the writer suggests that reasonable values of the coefficient of variation for each variable would be as shown in Table III.

TABLE III. Estimated coefficients of variation

\begin{tabular}{c|c}
\hline Variable & Estimated coefficient of variation \\
\hline & 0.10 \\
\hline$C_{y} / C_{2}$ & 0.20 \\
$f_{0}$ & 0.20 \\
$\eta_{1}$ & 0.15 \\
& 0.15 \\
& 0.25 \\
& 0.10 \\
\hline
\end{tabular}

The values suggested in Table III are estimates only. In quoting values for the coefficients of variation of $\beta$ and $f_{o}$, it is assumed that the estimates of $\beta$ and $f_{o}$ have resulted from an analysis of the particular structure rather than some empirical relationship (such as $f_{o} \sim 10 / N, N=$ No. of stories). The coefficients of variation of $\alpha, i, \nu, L_{z}, C_{z}$, and $C_{y} /$ $C_{z}$. are estimates based on the scatter observed in measurements of these quantities obtained over similar terrains and at similar heights. The coefficient of variation in the damping parameter $(\eta)$ is based on the scatter of field measurements obtained for similar types of structure, e.g. the scatter in measurements on concrete chimneys or the scatter for steel structures. In the case of most of the variables involved the number of field measurements available is very limited and the assigned coefficients of variation are largely subjective rather than the results of rigorous statistical analysis.

The coefficient of variation of a gust factor $G_{o}$ determined by values of the independent variables ( $\alpha=\alpha_{o}, i=i_{o}$ etc.) can be expressed as,

$$
C_{G_{o}} \simeq \sqrt{\omega_{\alpha_{o}}^{2} \cdot C_{\alpha}{ }^{2}+\omega_{i_{o}}{ }^{2} C_{i}{ }^{2}+\cdots}
$$

in which

$$
\begin{aligned}
& \omega_{\alpha_{0}}=\frac{\alpha_{o}}{G_{o}}\left(\frac{\partial G}{\partial \alpha}\right) \alpha_{o}, i_{o} \\
& \omega_{i_{0}}=\frac{i_{o}}{G_{o}}\left(\frac{\partial G}{\partial i}\right) \alpha_{o}, i_{o}, \cdots \quad \text { etc. }
\end{aligned}
$$

and $C_{\alpha}, C_{i}$, etc., are the coefficients of variation of $\alpha, i$, etc

The weights $\omega_{\alpha_{o}}, \omega_{i_{o}}$ etc. and the resulting value of $C_{G_{o}}$ have been computed for three structures and the results are presented in Table $V$. The three structures examined range from a comparatively stiff structure for which resonant effects are negligible to a tall lightly damped structure of low natural frequency and a dynamic response dominated by resonance. The properties of the three structures and the relevant meteorological data are presented in Table IV.

For all three structures it is apparent that accuracy depends largely on the specification of the intensity of turbulence. In the structure "A," for which resonant effects are small, the errors introduced by poorly defined data are due almost entirely to the definition of the intensity of turbulence. As the contribution of resonant effects increases, the influence of other parameters is of increasing importance. The specification of $C_{Z}, C_{y} / C_{Z}, \nu, L(h)$ and $f_{o}$ are of approximately equal importance while the specification of damping is somewhat more significant. In no case is the gust factor particularly sensitive to the choice of the mode shape or the mean velocity profile. The coefficient of variation associated with the variability of data is approximately 0.10 and, as could be anticipated, increases with an increase in the relative significance of resonant effects. 
TABLE IV. Structural and meteorological data

\begin{tabular}{|c|c|c|c|}
\hline \multirow[b]{2}{*}{ Description } & \multicolumn{3}{|c|}{ Structure } \\
\hline & $\begin{array}{c}\text { "A" } \\
\text { R.C. framed } \\
\text { apartment } \\
\text { block }\end{array}$ & $\begin{array}{c}\text { "B" } \\
\text { R.C. frame } \\
\text { office } \\
\text { block }\end{array}$ & $\begin{array}{c}\text { "C" } \\
\text { Steel frame } \\
\text { office } \\
\text { block }\end{array}$ \\
\hline $\bar{u}_{.}(H) \ldots \ldots$ & $100 \mathrm{ft} / \mathrm{sec}_{\ldots} \ldots$ & $120 \mathrm{ft} / \mathrm{sec}_{\ldots}$ & $150 \mathrm{ft} / \mathrm{sec}$ \\
\hline Exposure.... & Suburban & City $\ldots \ldots \ldots$ & City \\
\hline$B$ & $150 \ldots \ldots$ & 150 & 200 \\
\hline$H_{--}$ & $200 \ldots \ldots$ & $600 \ldots$ & 1,200 \\
\hline$\beta \ldots \ldots$ & 1.0 & $1.0 \ldots \ldots$ & 1.0 \\
\hline$f_{0 \ldots} \ldots \ldots \ldots$ & $0.6 \mathrm{cps} \ldots \ldots$ & $0.2 \mathrm{cps}_{\ldots} \ldots \ldots$ & $0.08 \mathrm{cps}$ \\
\hline$\eta \ldots \ldots \ldots$ & $0.020 \ldots$ & $0.02 \ldots$ & 0.015 \\
\hline$\alpha_{\ldots} \ldots \ldots$ & $0.25 \ldots \ldots$ & $0.36 \ldots$ & 0.36 \\
\hline$i(H) \ldots$ & $0.15 \ldots \ldots$ & $0.14_{\ldots}$ & 0.11 \\
\hline$L_{Z}(H)$ & $6,400 \mathrm{ft}_{\ldots} \ldots$ & $10,000 \mathrm{ft}_{\ldots} \ldots$ & $18,000 \mathrm{ft}$ \\
\hline$v\left(f_{o}\right)$ & $1 \ldots \ldots$ & $1 \ldots \ldots$ & 1 \\
\hline$C_{Z \ldots \ldots} \ldots$ & $10 \ldots \ldots$ & $10 \ldots \ldots$ & 10 \\
\hline$C_{B} / C_{Z} \ldots \ldots$ & 1.6 & 1.6 & 1.6 \\
\hline$g_{-\ldots} \ldots$ & $3.5 \ldots$ & $3.5 \ldots$ & 3.50 \\
\hline$r_{\ldots} \ldots \ldots$ & 0.33 & 0.32 & 0.25 \\
\hline$B \ldots \ldots$ & $0.70_{\ldots}$ & 0.68 & 0.68 \\
\hline$S F / \eta \ldots$ & 0.085 & $0.48 \ldots$ & 1.84 \\
\hline$G_{\ldots} \ldots \ldots$ & 2.04 & $2.21 \ldots \ldots$ & 2.42 \\
\hline
\end{tabular}

TABLE V. Coefficients of variation of gust factor estimates for structures $A, B, \& C$

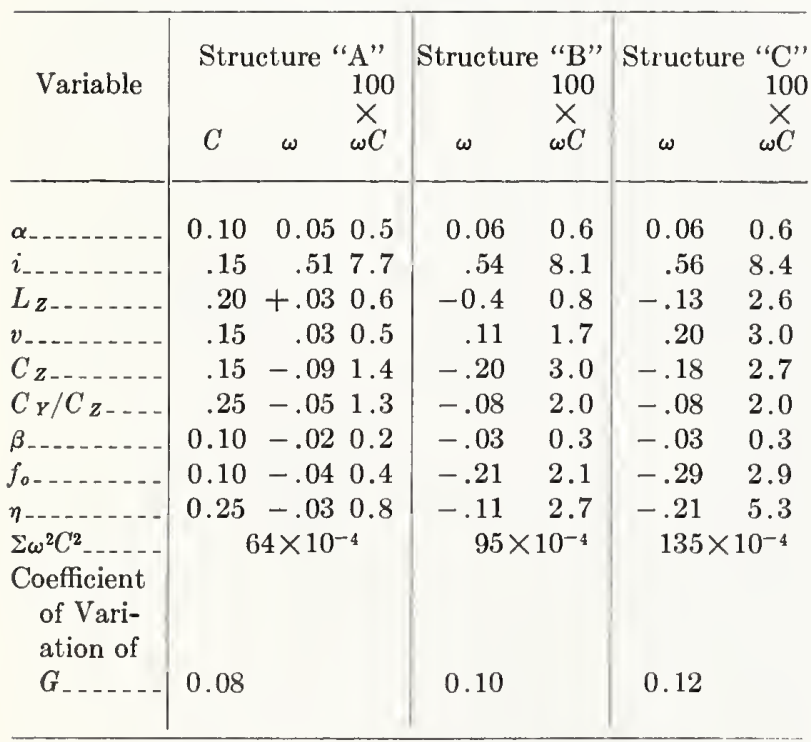

\section{A Comparison of Gust Factors Computed by the Various Proposed Methods}

Gust factors have been computed for three structures, in open and urban terrain and for two values of structural damping. The properties of the buildings and the flow conditions are presented in Table
VI. The computed values of the gust factor are presented in Table VII. In order to maintain uniformity the "peak factor" $g$ has been taken as 3.5 in all computations. The wind speed chosen in each case corresponds to a gradient wind speed of approximately $150 \mathrm{ft}$./sec.

The gust factors computed in accordance with Section 1 of this paper will be taken as reference values and nominally correct. The gust factors as computed by the method of Davenport are, on average, low by $5 \%$ while those computed by the method of Vickery are, on average, $5 \%$ high. In view of the accuracy estimates of the previous sections these discrepancies are barely significant. It should be noted that in neither case are the methods employed and attributed to Davenport and Vickery identical with those proposed in reference [1] and [2]. A modified form of Davenport's original proposal was prepared for studies conducted in connection with the Building Code of Canada and it is this modified form that has been adopted. A modified form of Vickery's proposal has also been adopted, this modified form will be published as part of a discussion [15] on the paper by Vellozzi and Cohen [3].

The gust factors computed by the method proposed by Vellozzi and Cohen are, on average, $17 \%$ low while for the larger structures the discrepancy is approximately 25\%. This discrepancy is highly significant and can be attributed to the size reduction factor used by Vellozzi and Cohen. Vellozzi and Cohen evaluate the size reduction factor in a manner similar to that used in Section 2 of this paper but compute the correlation integral over a rectangular prism rather than a plane rectangle. The depth of the prism is taken as four times the least dimension of the frontal rectangle. The argument put forward in support of this approach is that a gust must envelop a large portion of the wake before it becomes fully effective. This may well be true in regard to wake pressures but not in regard to the more significant pressure fluctuations on the front face of a building. In comparison with experimental observations the size reduction factor proposed by Vellozzi and Cohen is a severe underestimate.

The differences between the methods proposed in references [1] and [2] and that proposed in this paper lie primarily in the size reduction factor and the form of the spectrum of velocity fluctuations. These differences are discussed in a contribution to the discussion on the paper by Vellozzi and Cohen. Although the differences are quite substantial it 


\begin{tabular}{|c|c|c|c|c|c|c|}
\hline & \multicolumn{2}{|c|}{ Building 1} & \multicolumn{2}{|c|}{ Building 2} & \multicolumn{2}{|c|}{ Building 3} \\
\hline Height.. & \multicolumn{2}{|c|}{150} & \multicolumn{2}{|r|}{500} & \multicolumn{2}{|c|}{1,200} \\
\hline Breadth.... & \multirow{2}{*}{\multicolumn{2}{|c|}{$\begin{array}{l}150 \\
\quad 1.0 \mathrm{cps}\end{array}$}} & \multicolumn{2}{|r|}{200} & \multicolumn{2}{|c|}{200} \\
\hline$f_{0+1}$ & & & & $0.2 \mathrm{cps}$ & & \\
\hline$\eta$ & \multirow{2}{*}{\multicolumn{2}{|c|}{$\begin{array}{c}0.01,0.02 \\
1\end{array}$}} & \multirow{2}{*}{\multicolumn{2}{|c|}{$\begin{array}{r}0.01,0.02 \\
1\end{array}$}} & \multirow{2}{*}{\multicolumn{2}{|c|}{$\begin{array}{c}0.01,0.02 \\
1\end{array}$}} \\
\hline 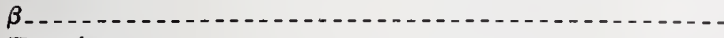 & & & & & & \\
\hline ( & URBAN & OPEN & URBAN & OPEN & URBAN & OPEN \\
\hline 年- & 0.35 & 0.14 & 0.35 & 0.14 & 0.35 & 0.14 \\
\hline$\sqrt{\overline{u^{\prime 2}}} / \bar{u}(h)$ & .245 & .125 & 0.16 & 0.11 & 0.12 & 0.93 \\
\hline$C_{Z} \ldots \ldots \ldots$ & 10 & 10 & 10 & 10 & 10 & 10 \\
\hline$C_{Y}-1$ & 16 & 16 & 16 & 16 & 16 & 16 \\
\hline$L(h)(\mathrm{ft})$. & 6,500 & 5,000 & 10,000 & 6,000 & 14,000 & 6,500 \\
\hline $\bar{u}(h) \mathrm{ft} . / \mathrm{sec}$ & 65 & 110 & 95 & 130 & 130 & 150 \\
\hline$C_{z} h / L(h)$ & 0.23 & 0.30 & 0.50 & 0.83 & 0.85 & 1.85 \\
\hline$C_{Z} f_{0} h / \bar{u}(h) \ldots$ & 23 & 13.6 & 10.5 & 7.7 & 9.2 & 8.0 \\
\hline - & 1.6 & 1.6 & 0.64 & 0.64 & 0.27 & 0.27 \\
\hline$f_{0} L(h) / \bar{u}_{-}$ & 100 & 45 & 21 & 9.2 & 10.7 & 4.3 \\
\hline 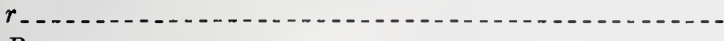 & 0.56 & .267 & 0.37 & .235 & 0.28 & .201 \\
\hline (2-1 & 0.71 & 0.67 & 0.68 & 0.60 & 0.64 & 0.51 \\
\hline$S_{-}$ & .010 & .025 & .026 & .101 & .140 & .330 \\
\hline$F_{-1}$ & .024 & .039 & .068 & .117 & .107 & .185 \\
\hline & .001 & .002 & .004 & .005 & .005 & .006 \\
\hline 其 & 3.5 & 3.5 & 3.5 & 3.5 & 3.5 & 3.5 \\
\hline$\frac{S F}{\eta}\left(\eta_{s}=.01\right)$ & .022 & .081 & .126 & .79 & 1.00 & 3.81 \\
\hline$\frac{S F}{\eta}\left(\eta_{s}=.02\right)$ & .011 & .044 & .074 & .47 & 0.60 & 2.35 \\
\hline$G=1+g r \sqrt{B}+\frac{S F}{\eta}$ & 2.67 & 1.83 & 2.16 & 1.97 & 2.26 & 2.45 \\
\hline $\begin{array}{l}\eta_{s}=0.01 \\
\eta_{s}=0.02\end{array}$ & & & & & & \\
\hline $13=-0.08--$ & 2.66 & 1.83 & 2.12 & 1.88 & 2.09 & 2.18 \\
\hline
\end{tabular}

TABLE VII. Comparison of computed gust factors

\begin{tabular}{|c|c|c|c|c|c|c|}
\hline & \multirow{2}{*}{ TERRAIN } & \multirow{2}{*}{ DAMPING } & \multicolumn{4}{|c|}{ Basis of calculation } \\
\hline & & & $\begin{array}{l}\text { Section } \\
\quad 2\end{array}$ & $\begin{array}{l}\text { Davenport } \\
\text { ref. (1) }\end{array}$ & $\begin{array}{l}\text { Vickery } \\
\text { ref. (2) }\end{array}$ & $\begin{array}{l}\text { Vellozzi \& Cohen } \\
\text { ref. (3) }\end{array}$ \\
\hline \multirow[t]{4}{*}{1} & Urban . . - & 0.01 & 2.67 & 2.57 & 2.87 & 2.59 \\
\hline & & 0.02 & 2.66 & 2.57 & 2.82 & 2.59 \\
\hline & Open.... & 0.01 & 1.83 & 1.74 & 1.85 & 1.72 \\
\hline & & 0.02 & 1.83 & 1.72 & 1.82 & 1.72 \\
\hline \multirow[t]{4}{*}{2} & Urban _. & 0.01 & 2.16 & 2.28 & 2.39 & 1.80 \\
\hline & & 0.02 & 2.12 & 2.10 & 2.28 & 1.80 \\
\hline & Open_-. & 0.01 & 1.97 & 1.93 & 2.13 & 1.56 \\
\hline & & 0.02 & 1.88 & 1.78 & 1.97 & 1.54 \\
\hline \multirow[t]{4}{*}{3} & Urban & 0.01 & 2.26 & 2.48 & 2.44 & 1.63 \\
\hline & & 0.02 & 2.09 & 2.08 & 2.23 & 1.61 \\
\hline & Open & 0.01 & 2.45 & 2.03 & 2.44 & 1.58 \\
\hline & & 0.02 & 2.18 & 1.95 & 2.17 & 1.52 \\
\hline
\end{tabular}


would appear, from the results presented in Table VII, that their influence of the gust factor is, on average, quite small. There could well be, however, circumstances in which this would not be the case. In particular, the method proposed by Davenport results in relatively large estimates of the contribution by resonance and underestimates of the background effects. This change in the distribution is of significance with respect to estimates of acceleration which depend more on resonance than on the background excitation.

\section{Conclusions}

(i) The ratio of expected peak values of wind induced stress of deflection to their average values can be predicted by the use of a gust factor to an accuracy of typically $5 \%$ to $10 \%$.

(ii) The gust factor relates only the overall loads in the direction of the mean wind. Lateral loads or local pressures are not predictable by the gust factor.

(iii) The method is suitable only for free standing structures and should not be applied to such structures as guyed masts or suspension bridges.

(iv) The accuracy or reliability quoted above applies only in instances where the basic data are well defined. When used in conjunction with data normally available at any given site the accuracy of the gust factor will be typically $10 \%$ to $15 \%$.

\section{References}

[1] Davenport, A. G., "Gust Loading Factors." J. Struet. Div., Proc. A.S.C.E., Vol. 93, No. ST3, June 1967.

[2] Vickery, B. J., "On the Assessment of Wind Effects on Elastic Structures.” C.E. Trans., Inst. Aust., pp. 183-192.

[3] Vellozzi, J., and Cohen, E., "Gust Response Factors." J. Struct. Div., Proc. A.S.C.E., Vol. 94, No. ST6, June 1968.
[4] Davenport, A. G., "The Spectrum of Horizontal Gustiness Near the Ground in High Winds." Quart. J., Roy. Met. Soc., Vol. 87, 1961.

[5] Davenport, A. G., "A Note on the Distribution of the Largest Value of a Random Function with Application to Gust Loading." Proc. I.C.E., Vol. 28, pp. 187-195, June 1964.

[6] Davenport, A. G., and Isyumov, N., "A Wind Tunnel Study for the United States Steel Building." Eng. Sc. Res. Rep. BLWT-5-67, The University of Western Ontario, November 1967.

[7] Davenport, A. G., and Isyumov, N., "A Wind Tunnel Study for a Proposed 53 Story Building for the SeattleFirst National Bank Building." Eng. Sc. Res. Rep. BLWT-1-67, The University of Western Ontario, 1967.

[8] Dalgliesh, W. A., Wright, W., and Schriever, W. R., "Wind Pressure Measurements on a Full Scale High-Rise Office Building." Paper No. 6, Wind Effects on Buildings and Structures, University of Toronto Press, 1968.

[9] Newberry, C. W., Eaton, K. J., and Mayne, J. R., "The Nature of Gust Loading on Tall Buildings." Paper No. 15, Wind Effects on Buildings and Structures, University of Toronto Press, 1968.

[10] Vickery, B. J., "A Model of Atmospheric Turbulence for Studies of Wind Loads on Buildings." Proc. 2nd. Aust. Conf. on Hyd. \& Fluid Mech., Aukland, N.Z., 1965.

[11] Smart, H. R., Stevens, L. K., and Joubert, P. N., "Dynamic Structural Response to Natural Wind." Paper No. 22, Wind Effects on Buildings and Structures, University of Toronto Press, 1968.

[12] Van Koten, H., "Wind Measurements on High Buildings in the Netherlands." Paper No. 25, Wind Effects on Buildings and Structures, University of Toronto Press, 1968.

[13] Vickery, B. J., "Load Fluctuations in Turbulent Flow." J. Eng. Mech. Div., Proc. A.S.C.E., pp. 31-46, Vol. 94, No. EMI, Feb. 1968.

[14] Vickery, B. J., and Davenport, A. G., "The Response to Wind Load of a $1200^{\prime}$ R.C. Stack for the American Electric Power Service Corporation.' Eng. Sc. Res. Rep. BLWT-4-68, The University of Western Ontario, August 1968.

[15] Vickery, B. J., "Discussion on 'Gust Response Factors' by Vellozzi, J., and Cohen, E.' To be published, Proc. A.S.C.E., J. Struct. Div. 
SESSION 4.

\section{RECENT ADVANCES IN DESIGN PROCEDURES AND CURRENT DEFICIENCIES}

Chairman: Mr. Edward Cohen

Ammann \& Whitney

New York, New York 10011 



\title{
THE TREATMENT OF WIND IN THE DESIGN OF VERY TALL IBUILDINGS
}

\author{
Leslie E. Robertson and Peter W. Chen \\ Skilling, Helle, Christiansen, Robertson \\ New York, New York 10017
}

\begin{abstract}
This paper deseribes briefly some highlights of a study of the wind effects for the design of the United States Steel Office Building in Pittsburgh, Pennsylvania. Three types of models were used in a boundary layer wind tunnel to obtain data of the wind effects on the building. The wind tunnel results are eombined with Weather Bureau data to obtain statistieal estimates of the design parameter's. These parameters include the envelopes of maximum deflections, the contours of maximum and minimum wind pressures on the exterior walls, and the expected number of eyeles of oseillation per year which exeeds specifie values of aceeleration and of deflection.
\end{abstract}

Key words: Aeroelastieity; buildings; dynamies; statistieal analysis; struetural engineering; wind loads; wind tunnel modeling.

\section{Introduction}

Research on the structure of wind has clearly shown the random and turbulent character of wind which varies with the height above ground and with the topographic features of the ground surface. Furthermore, a realistic estimation of the responses of the structures to natural wind has been shown by researchers to require dynamic and statistical concepts.

The firm of Skilling, Helle, Christiansen, Robertson has been engaged during the last few years in the design of several tall buildings. Among them is the United States Steel Office Building which is now under construction in Pittsburgh. These tall buildings have provided the opportunity to incorporate many of these new concepts in the treatment of wind effects during the design of those buildings.

A rendering of the United States Steel Office Building is shown in Figure 1. This building is triangular in plan with 64 occupied stories exclusive of basements and heliport, has a total of 78 framed levels, and its height is $839 \mathrm{ft}$ above the plaza. The area of each floor is $41,400 \mathrm{sq} f t$ to the building line.

\section{Scope of the Wind Study}

The program for the study of the wind effects on the United States Steel Office Building consists of three coordinated phases, as follows:

(a) Environmental study-the gathering and analyses of all pertinent data for wind velocity and temperature.

(b) Simulation by wind tunnel models - the topographic model to establish the wind structures appropriate for the testing of building models; the aeroelastic model for static and dynamic deflections; and the pressure model for local pressures and for cross-checking of results.

(c) Synthesis of environmental criteria with the results of the wind tunnel measurements.

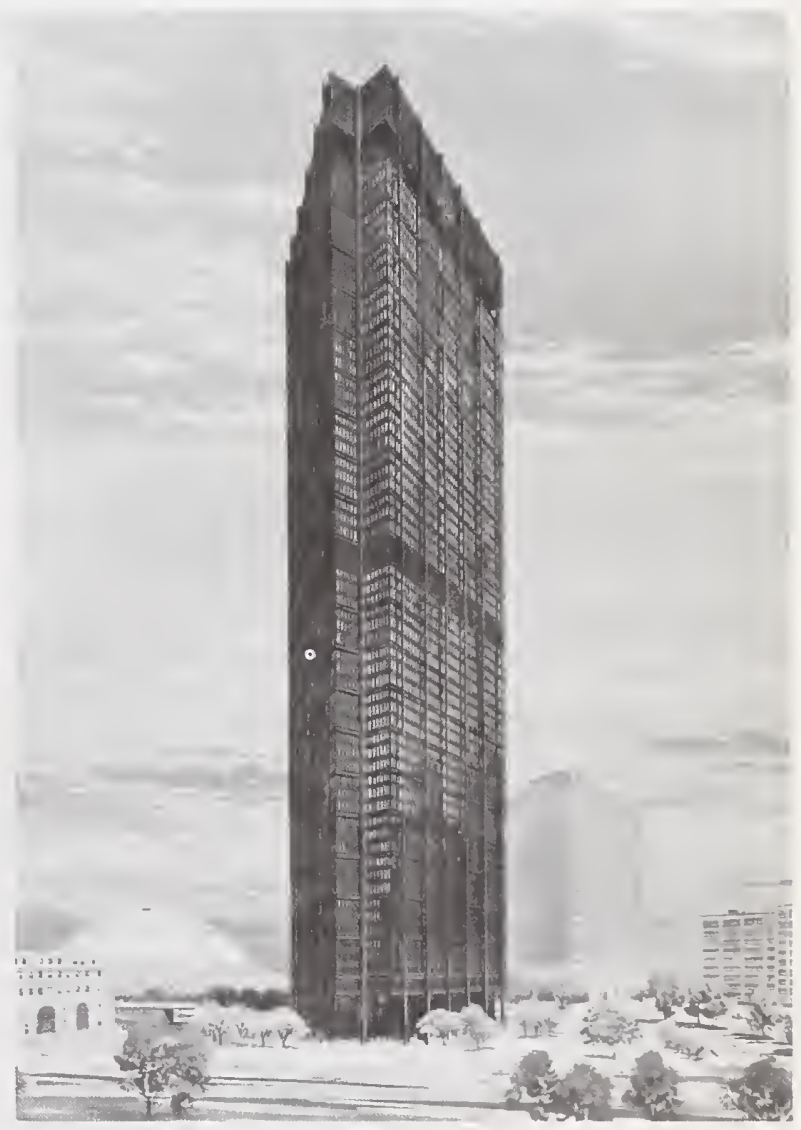

Figure 1. Rendering of United States Steel Offiee Building, Pittsburgh, Pennsylvania. 


\section{Environmental Study}

The most useful parameter that defines the wind climate of a locality is the mean gradient wind velocity taken over a time period between 5 minutes and 1 hour, as suggested by Professor Davenport [1]*. The interval that has been adopted is 20 minutes. Our task is then to determine the probability distribution of the annual maximum gradient wind velocity averaged over 20 minutes for the city in which the building will be built.

Three types of wind data were utilized, namely, the weather balloon data, the hourly observations, and records of annual maximum wind speeds. On the basis of the gradient wind speeds estimated from the three types of data, one probability distribution of the annual maximum gradient wind speeds is chosen as the design distribution [2]. This distribution is shown in Figure 2.

Wind and temperature data are combined in Figure 3 and Figure 4, to indicate the joint probability of the simultaneous occurrence of specific wind speeds and specific temperatures.

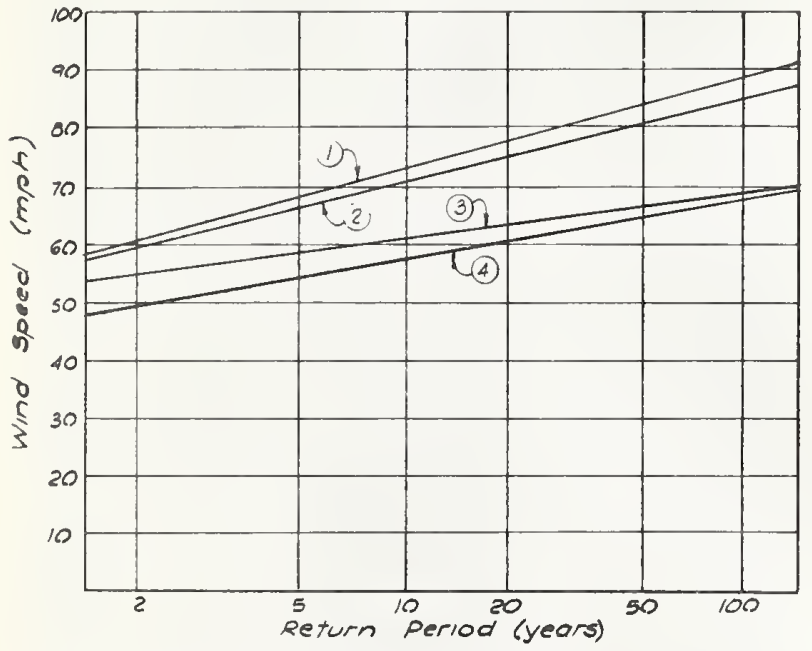

(1) Design distrioution based on dote of annual fostest mile speeds ot airports.

í Estimated from date of annud max 5 minute ove speeds of city office.

(3) Estimated from data of Wind Aloft Summary

ot 300 meters

(4) Estimated from data of hourly observations at eirport, $1956-1960$.

Frgure 2. Probability distribution of amnual maximum 20 minute average gradient wind speeds.

\footnotetext{
* Figures in brackets indicate the literature references at the end of this paper.
}

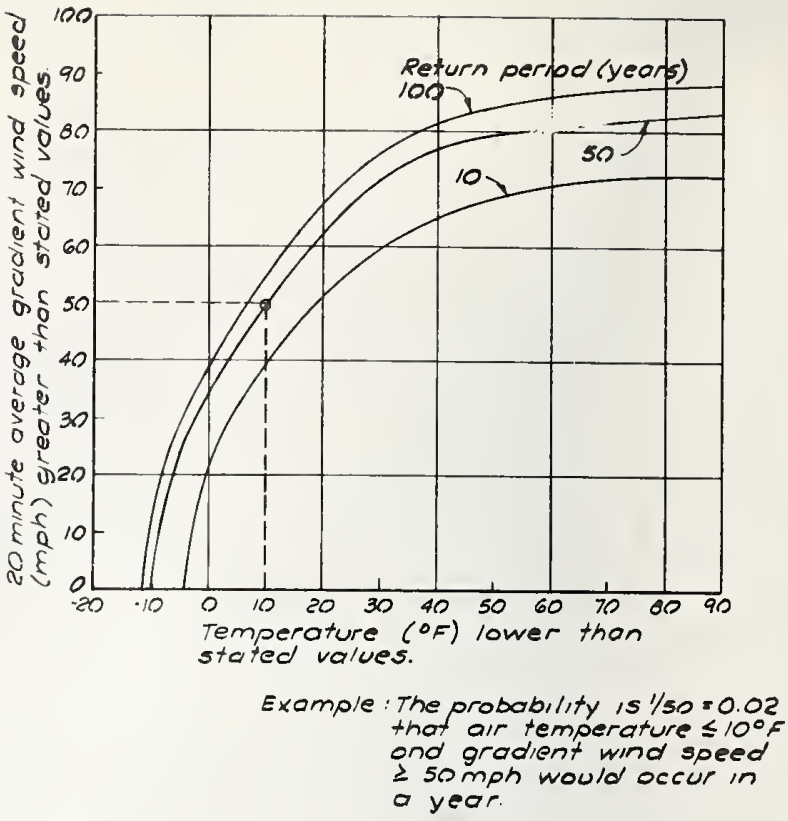

FrgURE 3. Joint probability of gradient wind speed and air temperature at site.

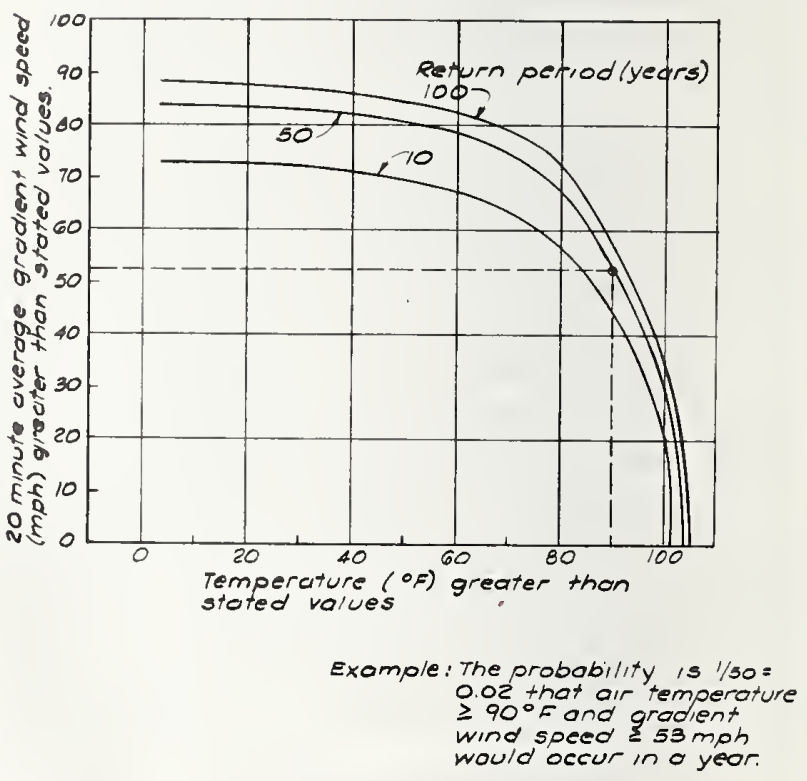

FIGURE.4. Joint probability of gradient wind speed and air temperature at site.

\section{Wind Tunnel Studies}

The wind tunnel studies consisted of four stages which were carried out at the Boundary Layer Wind Tunnel of the University of Western Ontario.

\subsection{Stage I}

During the preliminary concept stage of the design, various shapes of the building were con- 


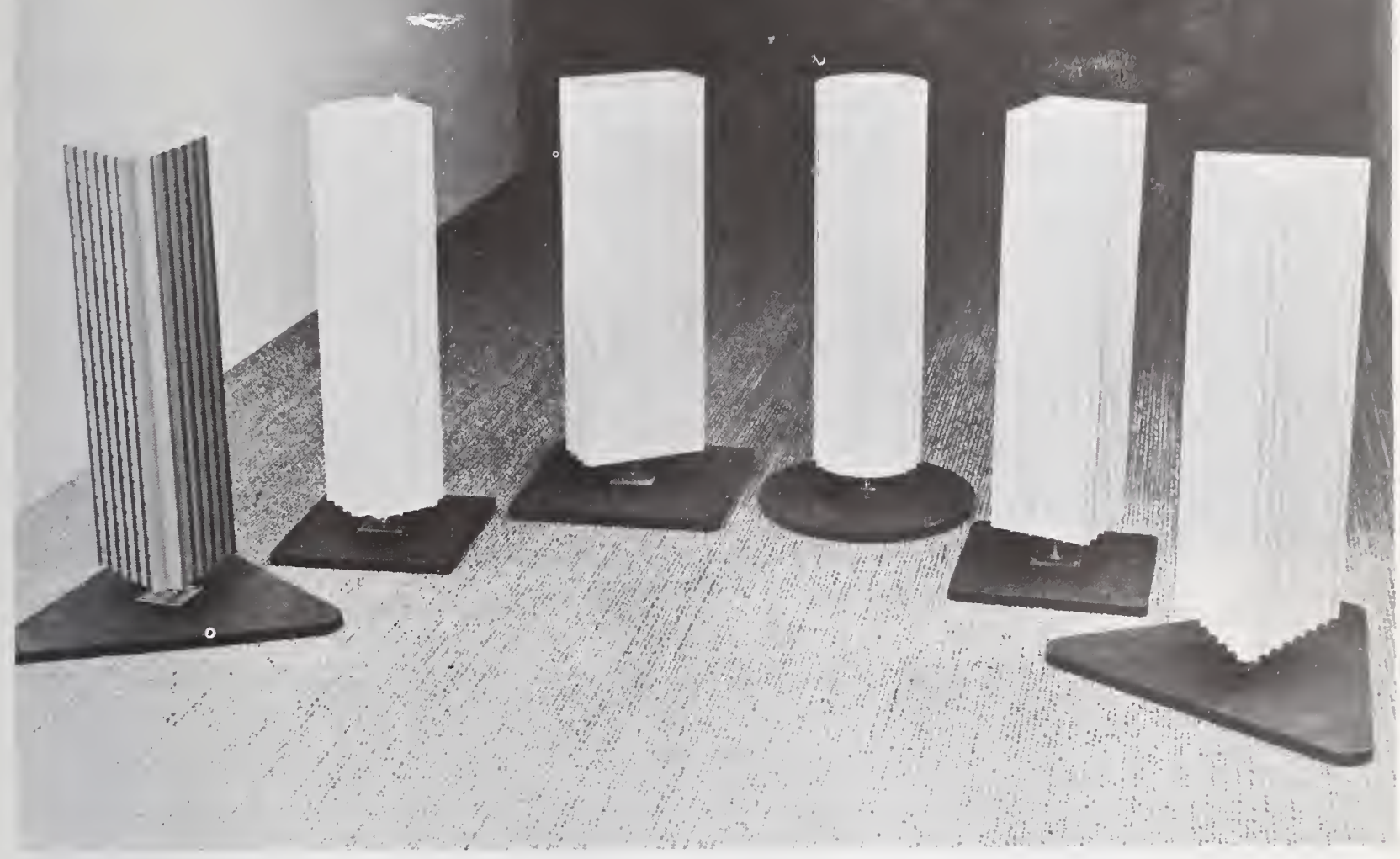

Figure 5. Models used in aeroelastic comparison study of building shapes.

sidered. In order to assess the relative aerodynamic merits of different building shapes, an aeroelastic study of six building shapes was made in a simulated city atmosphere. The six models that were tested are shown in Figure 5. The results are compared in Figure 6 by means of the envelopes of peak defleetions for all wind directions. The aerodynamie eharacteristics of the modified triangular building shape, which was preferred for struetural and architectural reasons, was found to be favorable and to be aerodynamieally superior to the eonventional rectangular shape-thus eonfirming its adoption for the final design of the United States Steel Offiee Building.

\subsection{Stage II}

A model of the Pittsburgh area was built to a scale of $1: 2000$ and tested in the boundary layer wind tunnel. Figure 7 shows a photograph of the topographieal model in the wind tunnel. The vertical profiles of mean veloeity and the profiles of intensity of turbulence at the building site were obtained for

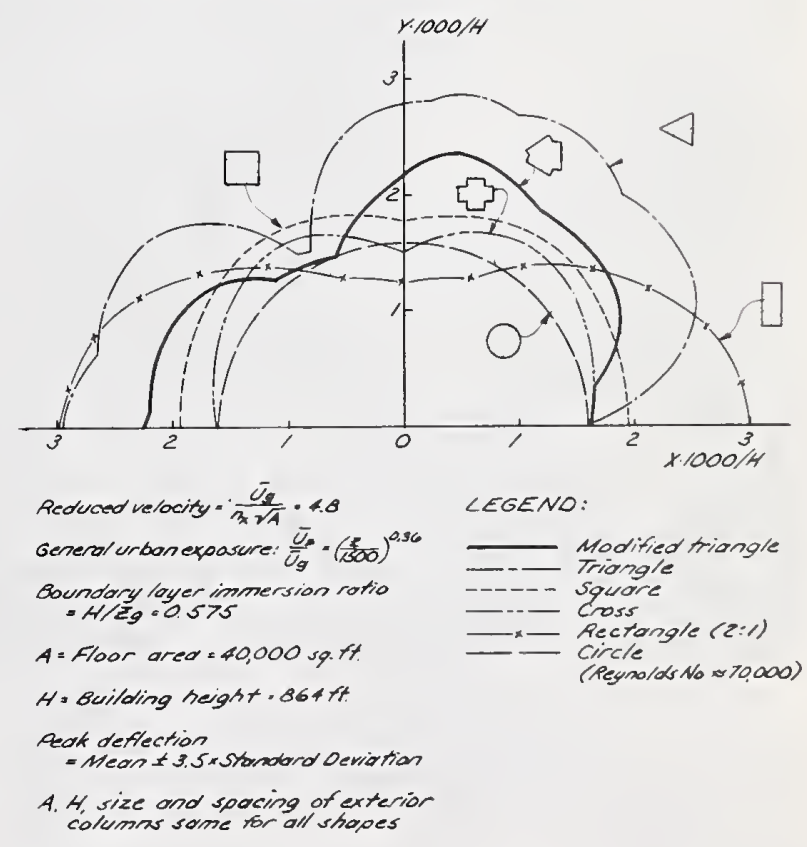

Figure 6. Envelopes of peak deflections of six building shapes. 


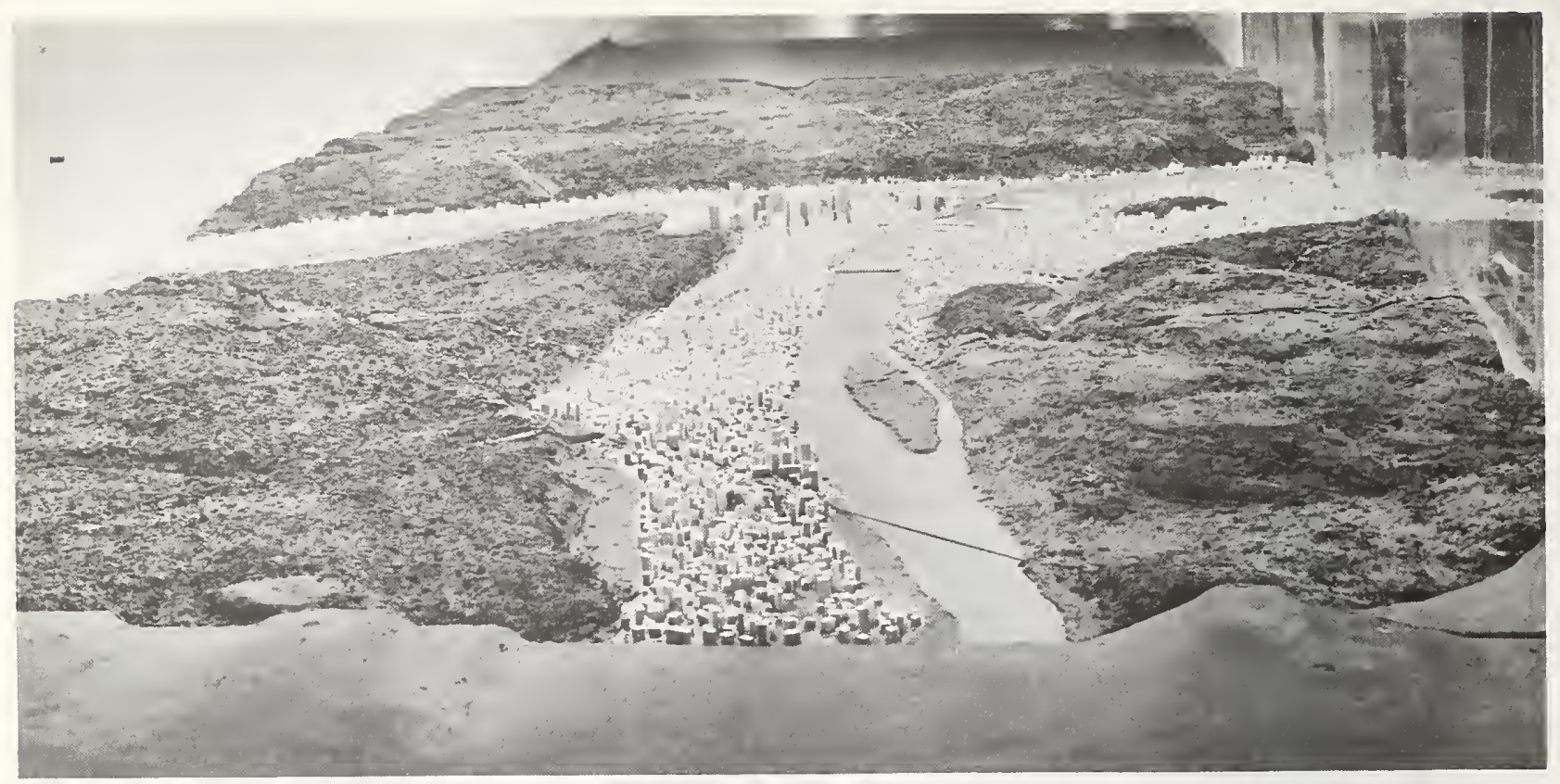

Figure 7. View of 1:2000 scale topographical model in wind tunnel.

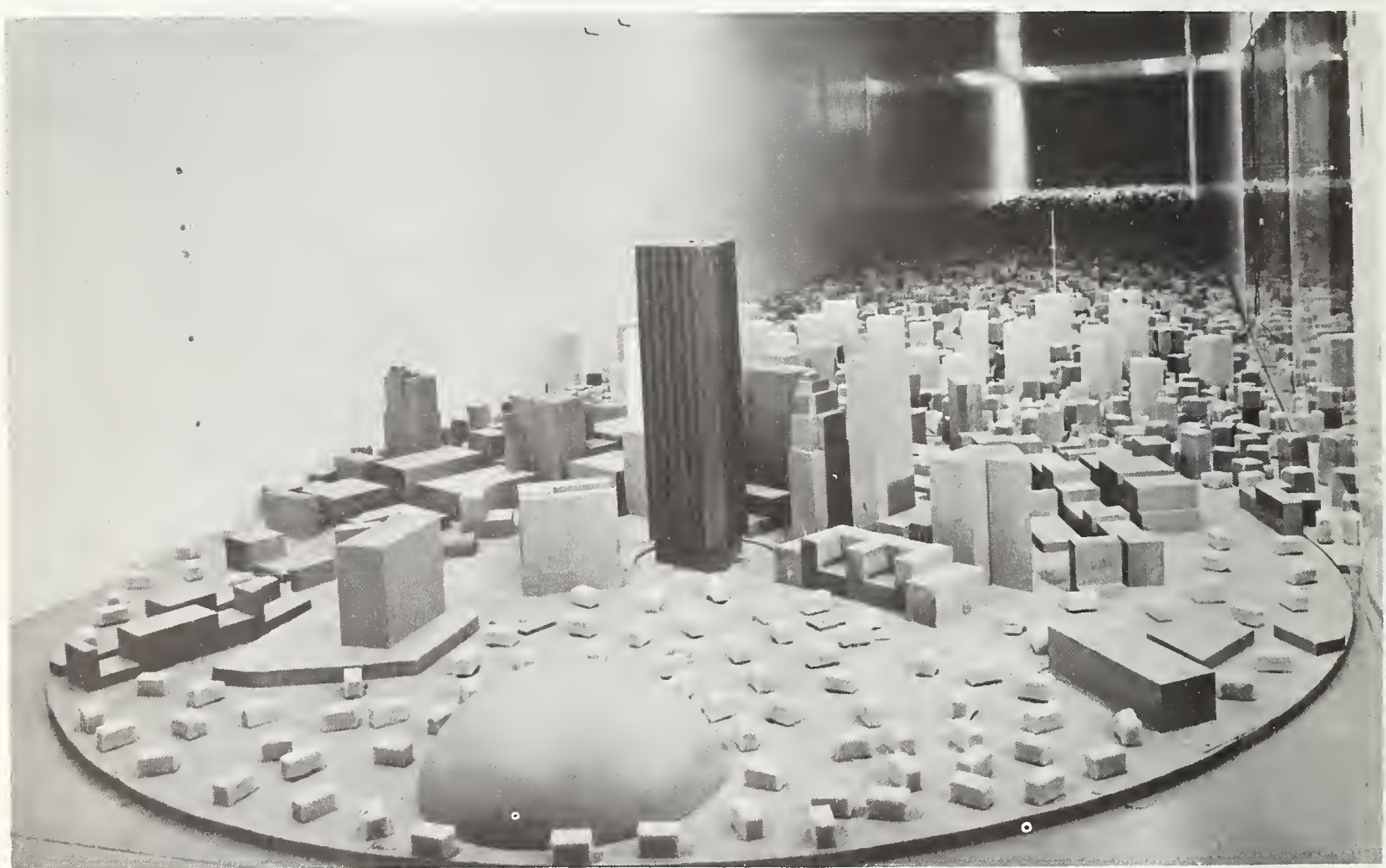

FIGURE 8. View of "rough" exposure showing 1:400 scale aeroclastic model of United States Steel Office Building.

the eight principal compass directions. These tests were completed in order to assess the extremes of exposure for later use in studying models of the building itself.

\subsection{Stage III}

The wind tunnel study of the final building design included both the measurements of the responses of 
the aeroelastic model and the measurements of the pressures on the exterior faces of the static model. The tests werc carried out in two extrcmes of exposure which had been established in Stage II. Figure 8 gives a view of the acroelastic model of the United States Steel Office Building in the boundary layer wind tunnel.

The aeroelastic responses of tall buildings are usually dominated by the fundamental modes of vibration which may be adequately approximated by a linear shape. Thus, the aeroelastic model of the building consists of a rigid model dynamically mounted so that the lowest mode of vibration is modelled in each of two transverse directions. The profiles of wind speeds are adjusted to match those found in the topographic model by varying the roughness of the floor surface of the wind tunnel. The buildings in the vicinity of the project site are reproduced to scale in the wind tunncl.

Measurements of the mean and of the RMS values of the deflection in two orthogonal axes are made for various angles of attack at different wind speeds and for various combinations of damping, building density, exposure and frequency ratio. Both static and dynamic deflections at the top of the building are plotted in Figures 9 and 10 for various wind direction. These two graphs clearly demonstrate the dynamic nature of the wind effects on the building.

The static model has pressure taps located at all points of interest such as the large glass curtain walls of the lobby. Figure 11 shows the static model with one of the faces opened. The mean and the RMS

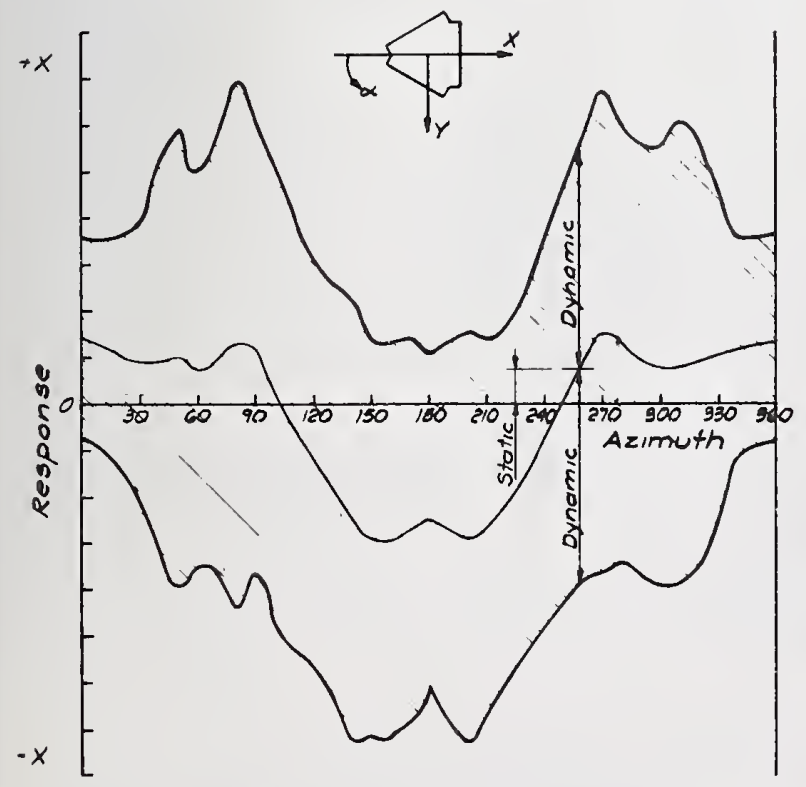

Figure 9. Static and dynamic responses along $X$ axis.

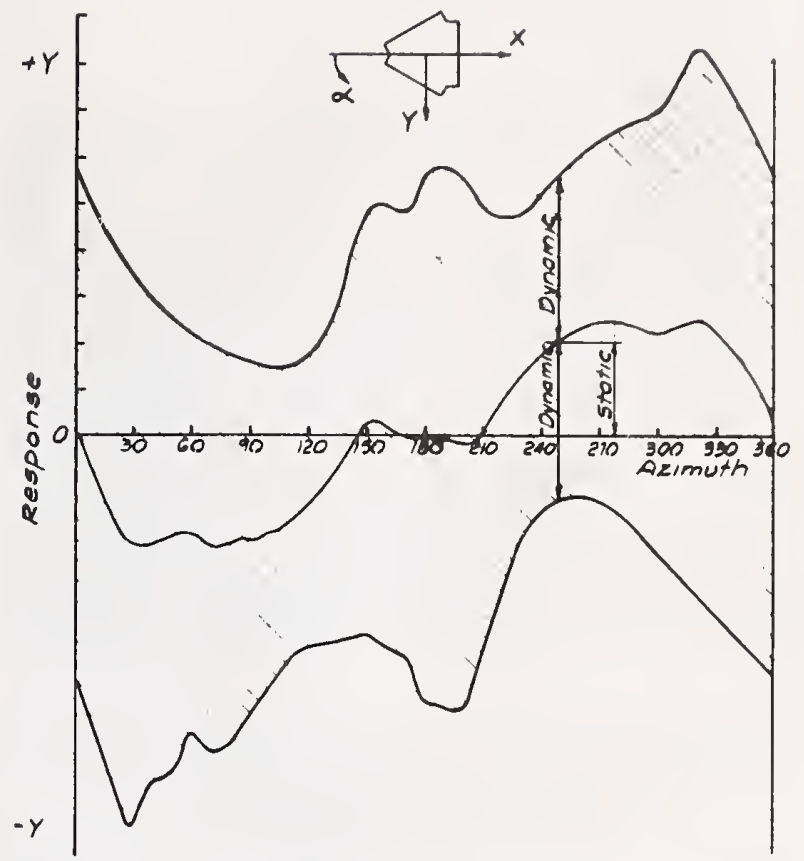

Figure 10. Static and dynamic responses along $Y$ axis.

value of the pressures are measured for various angles of attack and for two extremes of cxposure. The pressures arc converted into dimensionless form by dividing the local pressures by the dynamic pressure of the gradient level.

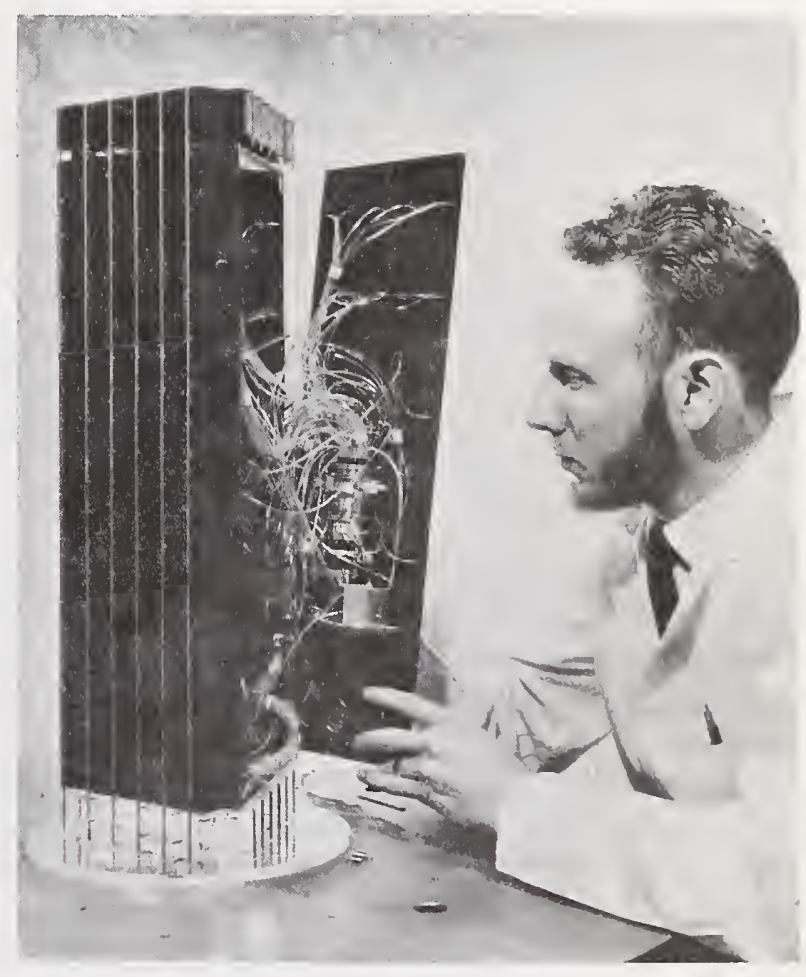

Figure 11. View of pressure model showing pressure sensing unit and piezometric pressure tap connections. 


\subsection{Stage IV}

The efficient operation of a rooftop landing field may be significantly influenced by the characteristics of the airflow in the region immediately above the building. Thus, the nature of the airflow over the proposed heliport atop the United States Steel Office Building was studied in detail by means of wind tunnel models [3]. The models were built to a scale of 1:96. Both flow visualization technique and hot-film anemometer sensor were used. It is found that the magnitude of the maximum turbulence intensity and the positions of the vortex layer over the United States Steel Office Building are similar to those over the Pan Am Building in New York City on which a heliport has been successfully operated.

\section{Statistical Prediction of Building Performance in Service}

Procedures have been developed in the last few years to make statistical estimation of various parameters of building performance in wind $[4,5,6]$. Such prediction is based on the probability distribution of the annual maximum gradient wind velocity and on the static and dynamic response characteristics of the building models obtained by tests in a boundary layer wind tunnel.

The parameters that are predicted include the maximum deflections, the maximum amplitudes of oscillation, the maximum and the minimum wind pressures on the exterior wall, and the expected number of cycles of oscillation per year which exceeds given values of deflection and of acceleration. These parameters are relevant to an evaluation of the building performance with respect to

(1) the stresses of the structural frames,

(2) the adequacy of the partitions and of the curtain wall; and

(3) the comfort of the building occupants.

Because of the directional characteristics of the wind effects on the building, the probability distributions of the parameters such as deflections and pressures are only indirectly related to the probability distribution of wind velocity. A specific value of deflection or some other parameter can arise from either a low wind speed coming from a critical direction or a higher wind speed coming from a non-critical direction. The procedures for estimating the combined probabilities of these events have been described in detail in the reference already cited. Some of the estimated results for the United States Steel Office
Building are shown in Figures 12 through 17. These figures show:

(a) Probability distributions of annual maximum deflection at top of the building (Fig. 12).

(b) Envelope of maximum deflections with 50 year return period (Fig. 13).

(c) Probability distribution of annual maximum peak amplitudes of oscillation at top of the building (Fig. 14).

(d) Expected number of cycles of oscillation per year having amplitudes exceeding specific values (Fig. 15).

(e) Maximum exterior pressure for wind speed of 50 year return period (Fig. 16).

(f) Minimum exterior pressure for wind speed of 50 year return period (Fig. 17).

\section{Conclusion}

A comprehensive treatment of the wind effects has been accomplished for the design of very tall buildings which incorporates the statistical and dynamical concept developed by the researchers in the recent years.

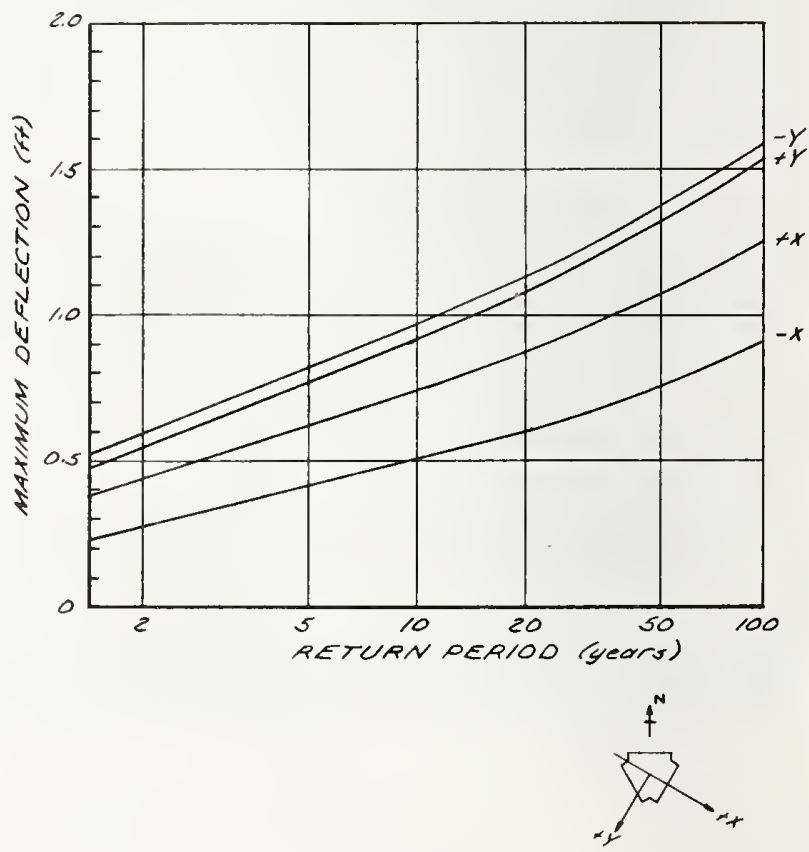

Figure 12. Probability distributions of annual maximum defiections at top of United States Steel Office Building in Pittsburgh. 


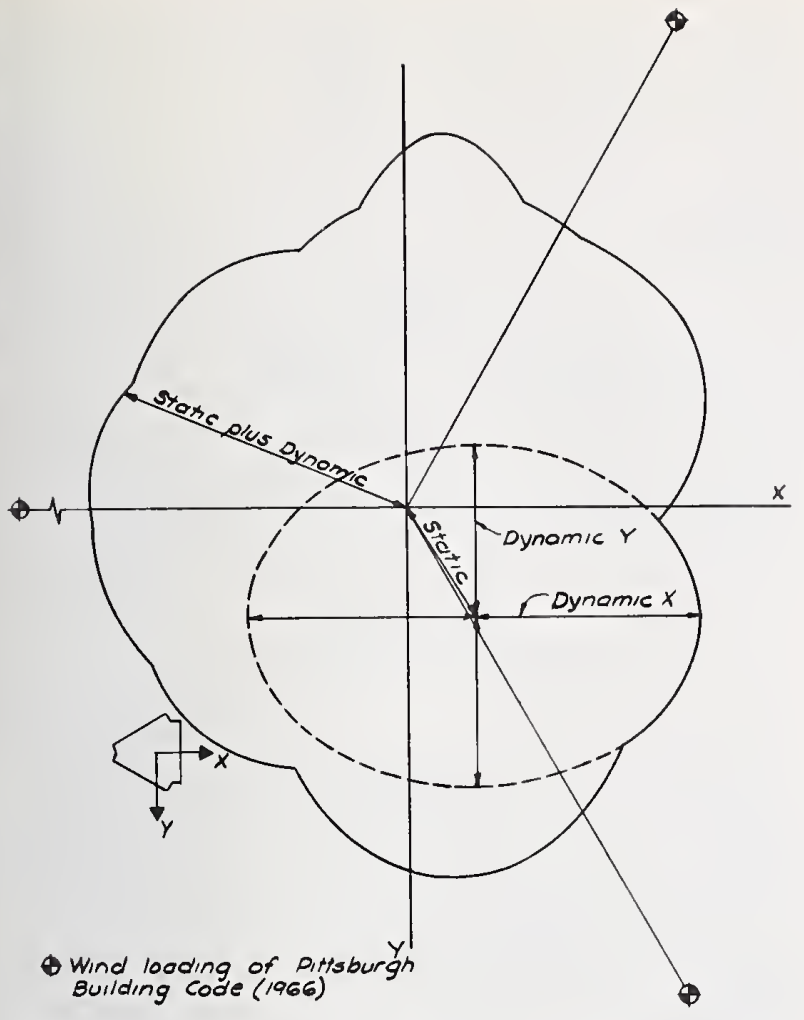

Figure 13. Envelope of maximum static and dynamic deflections (50 year return period).

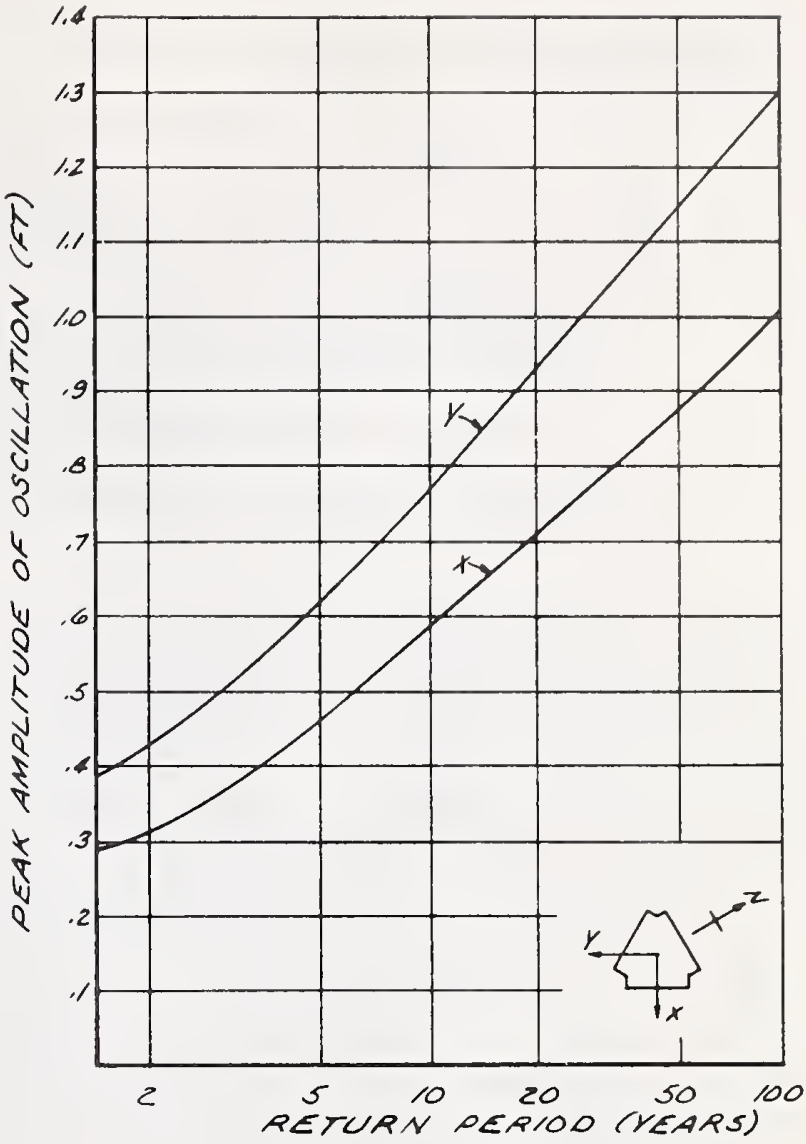

FIgURe 14. Probability distribution of annual maximum peak amplitudes of oscillation at top of United States Steel Office Building.

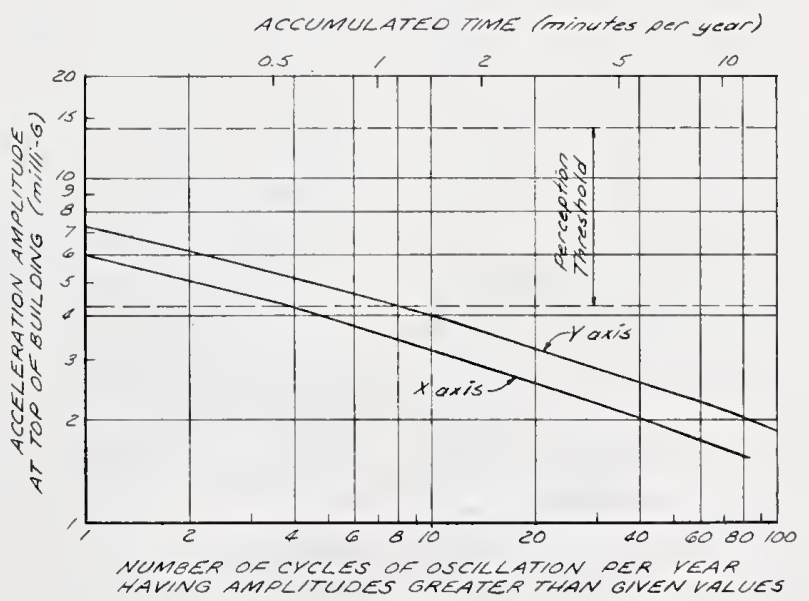

Figure 15. Expected number of cycles of oscillation per year having amplitudes exceeding specfic values. 


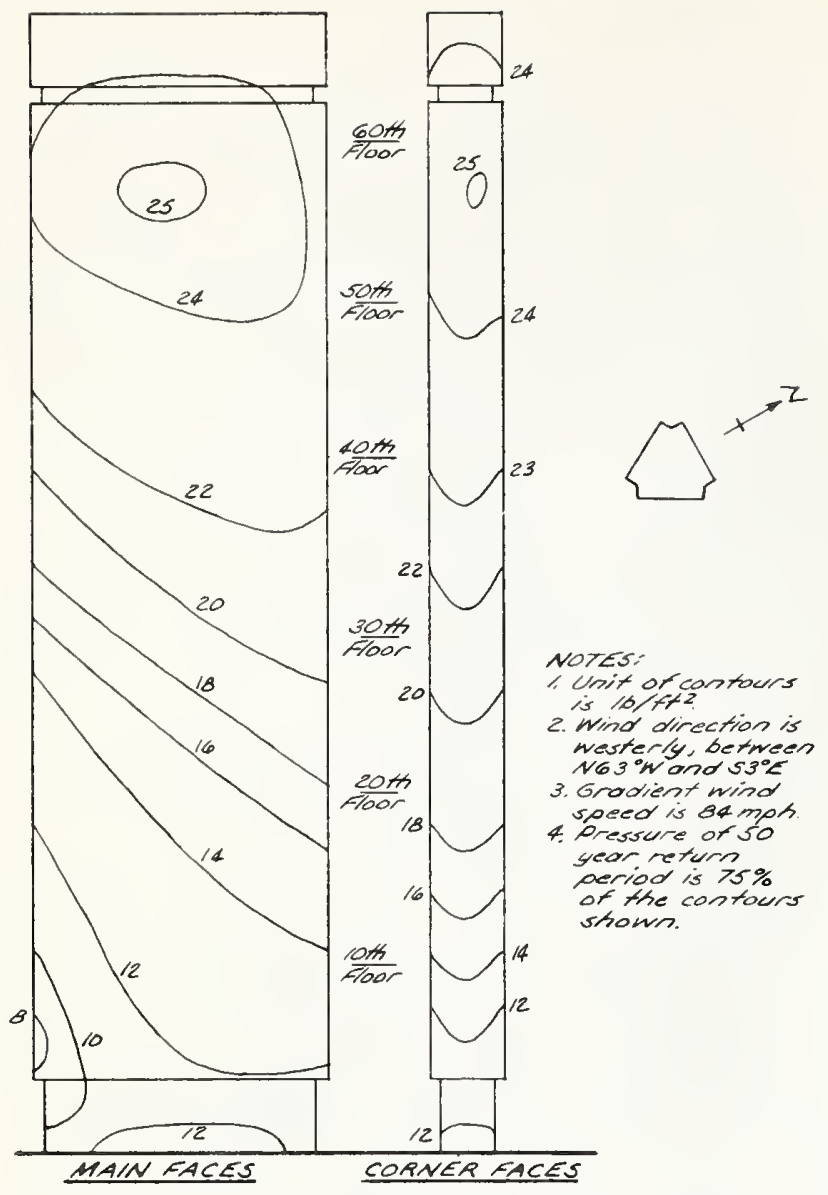

Figure 16. Maximum exterior pressure for wind speed of 50 year return period.

\section{References}

[1] Davenport, A. G., "The Relationship of Wind Structure to Wind Loading," Proceedings of the Symposium: Wind Effects on Buildings and Structures, National Physical Laboratory, June 1963, Her Majesty's Stationary Office, London 1965.

[2] Wind and Temperature-The United States Steel Office Building, A Report of Skilling, Helle, Christiansen, Robertson, to The United States Steel Corporation, March 1968.

[3] A Wind Tunnel Investigation For A Rooftop Landing Field-The United States Steel Office Building, A Report of Skilling, Helle, Christiansen, Robertson, to The United

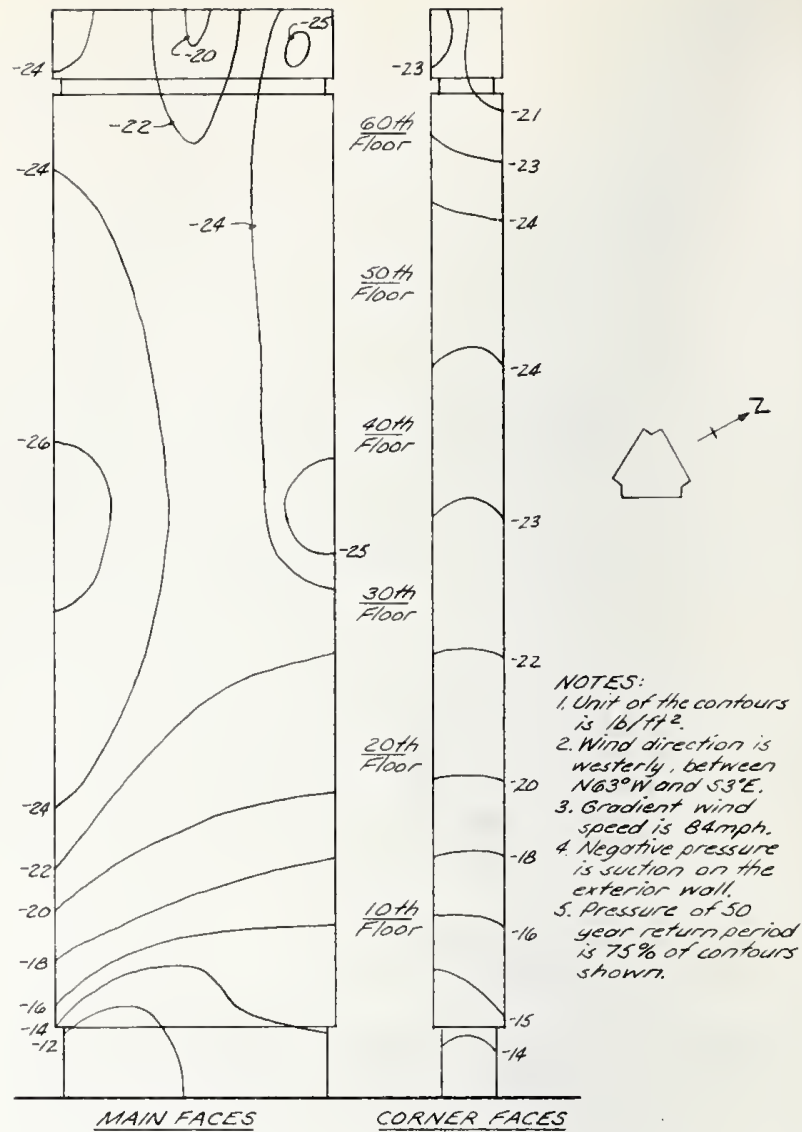

Figure 17. Minimum exterior pressure for wind speed of 50 year return period.
States Steel Corporation, April 1968.

[4] Davenport, A. G., "The Estimation of Load Repetitions on Structures with Application to Wind Induced Fatigue and Overload." Rilem International Symposium on the Effects of Repeated Loading of Materials and Structures, Mexico City, September 15-17, 1966.

[5] Robertson, L. E., "A Study of the Wind Effects on the Seattle First National Bank Building," Notes from a Design Seminar on Buildings in Earthquake and Wind, pp. 9.1-9.49, A.S.C.E., 1967.

[6] Robertson, L. E. and Chen, P. IV., "Glass Design and Code Implications for Extremely Tall Buildings," Building Research, pp. 6-11, May-June 1967, Building Research Institute, Washington, D.C. 


\title{
DYNAMIC RESPONSE OF TALL FLEXIBLE STRUCTURES TO WINID LOADING
}

\author{
Joseph Vellozzi and Edward Cohen \\ Ammann \& Whitney \\ New York, New York 10011
}

\begin{abstract}
The purpose of this paper is to present and discuss methods of calculating the dynamic response of tall, flexible structures, such as towers, stacks and masts, to wind loading. The paper is presented in two parts; the first part deals with the dynamic response of eylindrical struetures to vortex shedding and the second part deals with dynamic response to gust loading.
\end{abstract}

Key words: Aeroelasticity; buildings; circular cylinders; gust factors; masts; structural engineering; towers; vortex shedding; wind loads.

\section{Vortex Shedding}

\subsection{General}

A cylindrical structure subjected to wind stream normal to its axis vibrates in a lateral direction (transverse to the direction of the wind) due to the formation of vortices on alternating sides. The resulting pattern of flow on the downwind side is known as the von Karman Vortex Street [1]*. In a certain range of wind velocity and cylinder diameter these vortices are formed and shed from opposite sides of the cylinder at a regular frequency; consequently, alternating differential pressures are exerted upon the cylinder by the moving fluid. When the vortex frequency approaches the frequency of one of the normal modes of vibration of the structurc, resonant oscillation results due to the alternating pressures. The frequency of the oscillation remains close to the natural frequency of the structure. The alternating pressures also cause oscillations in the direction of the wind strcam but the major oscillations are transverse to the wind. The oscillations will continue to build until the energy dissipated by the damping in the structure equals the energy supplied by the wind stream. Hence, lightly damped structures are especially susccptible to this phenomenon.

The manner in which vortices are shed from a cylindrical structure depends upon the Reynolds number, $N_{R}$, where:

$$
N_{R}=U d / \nu
$$

where $U$ is the velocity of the wind, $\nu$ is its kinematic viscosity, and $d$ is a characteristic dimension such as the diamcter of the cylinder. At extremely small

* Figures in brackets indicate literature refercnces at the end of this paper.
Reynolds numbers the flow in the immediate vicinity of the cylinder is very much like ideal flow and no vortices are formed. At somewhat higher values, up to $N_{R}=40$, the flow separates from the surface and forms two symmetrical stationary vortices [2]. Above $N_{R}=40$, however, the stationary vortices become distorted and unstable, and an asymmetrical flow condition develops wherein vortices are formed at opposite sides of the cylinder. These vortices grow in strength in the vicinity of the cylinder and move downstrcam with the flow in an alternating pattern as they detach from the cylinder. The flow in the vortex street is viscous from $N_{R}=40$ to approximately 150 , and the vortices decay by viscous diffusion [2]. The range from $N_{R}=150$ to 300 is a transition region in which the flow changes from laminar to turbulent in the frec vortex layer which separates from each side of the cylinder [3]. Above $N_{R}=300$, the flow becomes turbulent at the separation point. In this range, called the irrcgular range, the vortex shedding is periodic, but the velocity in the immediate vicinity of the cylinder fluctuates in an irregular manner because of the flow turbulence. The irregular manner in which the velocity fluctuates is augmented by the turbulence in the natural wind. The result of these fluctuations is that the amplitudes of the lift or side forces on the cylinder are more or less random, and the randomness becomes more pronounced with increasing Reynolds numbers.

The frequency of the vortex shedding is fairly periodic for Reynolds numbers in the range of about 40 to $3 \times 10^{5}$. At or near the latter Reynolds number, the flow in the boundary layer on the forward portion of the cylinder changes from laminar to turbulent, and the separation points move rearward on the cylinder. There follows an abrupt drop in the drag coefficient and the wake becomes narrower and apparently aperiodic [4]. The result is that the 
frequency of the vortex shedding and the amplitudes of the lift forces are both random. Again, the natural wind turbulence augments this randomness. The frequency of the vortex shedding rcmains random with increasing Reynolds numbers up to about $3.5 \times 10^{6}$; however, with increasing Reynolds numbers above $3.5 \times 10^{6}$, the frequency of vortex shedding undergoes another change whereby there is a gradual return to definite periodicity $[5,6]$.

The frequency at which vortices are shed from a cylinder is usually expressed non-dimensionally as the Strouhal number $S$ :

$$
S=\frac{f d}{U}
$$

where $f$ is the frequency of the shedding, $d$ is a typical dimension and $U$ is the wind speed. When the vortex shedding is periodic, $f$ is the frequency of this shedding; however, when the shedding is random, it is necessary to speak of a power spectrum, or a correlation function, rather than a single dominant frequency. For random vortex shedding, an aerodynamic Strouhal number is sometimes defined by, "the predominant frequency measured from an autocorrelation function (or the peak power of the power spectrum) of the unsteady aerodynamic lift forces" $[5]$.

The variation of the Strouhal number, $S$, with Reynolds number, $N_{R}$, is shown in Figure 1. It is noted that in the Reynolds number range less than $3 \times 10^{5}$, where the vortex shedding is periodic, the value of the Strouhal number is fairly constant and in the order of 0.2 , the value generally accepted for the circular cylinder. For greater Reynolds numbers in

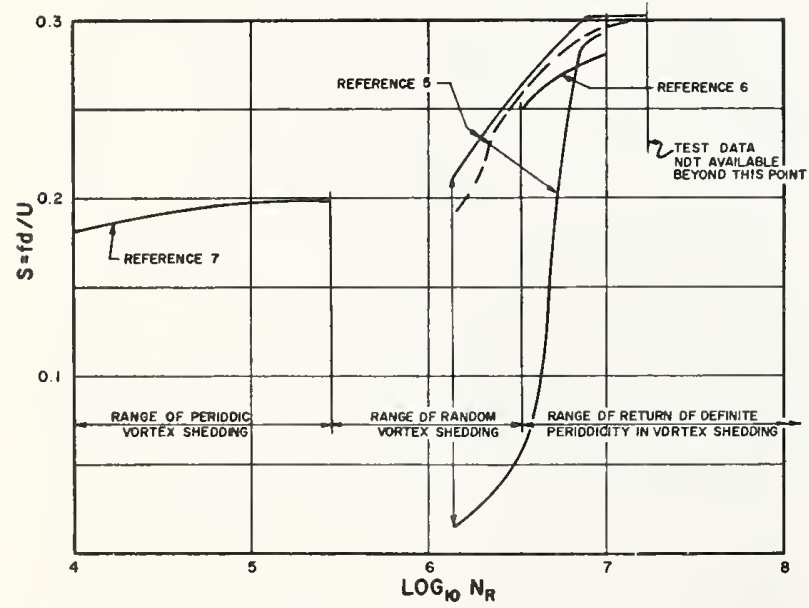

Figure 1. Variation of Strouhal number with Reynolds number for circular cylinder. the range of $3 \times 10^{5}$ to $3.5 \times 10^{6}$, where the vortex shcdding is random, there is a gradual rise in the "aerodynamic" Strouhal number, and with increasing Reynolds numbers above $3.5 \times 10^{6}$, where there is a gradual retur'n of definite periodic vortex shedding, the Strouhal number increases to a constant value of about 0.3 . In the range where the vortex shedding is random, the Strouhal number determincd from an autocorrelation function, which is esscntially the center frequency point of the power spectral density, is shown as a dashed line; the effective bandwidth of the unsteady force is presented in terms of the Strouhal number by the solid lines. The characteristic narrowing of the Strouhal number bandwidth with increasing Reynolds numbers above $3.5 \times 10^{6}$ is an indication of the return of periodicity in the vortex shedding at these Reynolds numbers.

For tapered structures where both the diameter and wind spced $U$ vary with height, the Strouhal number will likewise vary with height. Some recent experiments on this variation for tapered stacks [7] have indicated that the tendency is for the frequency of the vortex shedding to be constant over a substantial part of the axial length at a value determined by the wind speed and diameter at the top. In other words, the frequency of vortex shedding on a tapered cylinder would be "locked in," at least for a considerable length of the height, to the Strouhal frequency appropriate to the least diameter. These experiments also suggest that the top diameter of cylindrical towers and stacks should be as small as possible since critical wind speed and excitation (which varies with $U^{2}$ ) is thereby kept to a minimum, and less structural damping is needed to prevent oscillations.

When a cylindrical structure is vibrating in resonance with periodic vortex shedding forces, the vibration itself causes an increasc in the magnitude of the forces. The additional aerodynamic force is probably associated with a periodic shifting of the separation points of the flow from the surface of the structure, thereby causing increased circulation. At the same time, the vortices in the wake are larger and stronger than would be the case if the cylinder were stationary. Steinman [16] hypothesized that if vortices are shed at or near each end of the amplitude range, the normal width of the vortex street is increased by the ratio

$$
\frac{h+2 y}{h}=1+\frac{2 y}{1.3 d}
$$

in which $h(\approx 1.3 d)$ is the width between the centers of the two rows of vortices behind a non-oscillating 
cylinder, $y$ is the half amplitude of vibration, and $d$ is the diameter of the cylinder. For the vortices to be stable, the distance between the vortices in each row must also increase in the same ratio. Figure 2 (after Weaver [17] shows schematically the increased dimensions of the vortex street due to vibration of the cylinder. The increase in distance between shedding vortices decreases the shedding frequency and the Strouhal number by the reciprocal of Equation 3. Hence, resonant vibrations of large amplitude cannot be attained when the Strouhal frequency for a stationary cylinder exactly equals the natural frequency of an elastic body. In this instance, as soon as oscillation begins, the Strouhal frequency decreases and goes out of phase from the natural frequency. However, when oscillation begins at a wind speed slightly higher than the critical velocity, the Strouhal frequency decreases to the point where it matches the natural frequency, and the vibrations are sustained or increased. The ratio of the velocity $U_{p}$ at which peak amplitude occurs to the critical velocity based on $S=f d / U$ for a stationary cylinder is in the same ratio as the dimensional increase in the vortex street; i.e.

$$
U_{p} / U=1+1.54 y / d
$$

Similarly, the ratio between the Strouhal number $S_{p}$ at which peak vibration occurs and the Strouhal number $S$ for a stationary cylinder is

$$
S p / S=\frac{1}{1+1.54 y / d}
$$

It has been suggested by Steinman [16] and others [18] that the limiting ratio of $y / d$ is unity, and this is generally supported by experiments in which structural damping is very small.

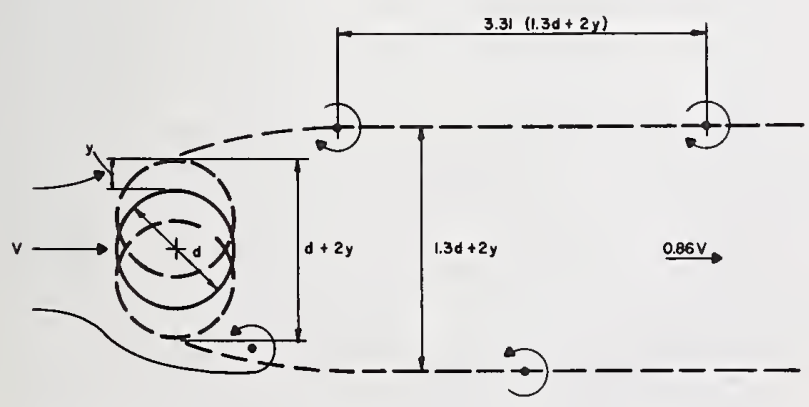

Figure 2. Dimensional increase in vortex street due to vibration (after Weaver).

\subsection{Stochastic Excitation}

The Reynolds numbers of primary interest for cylindrical towers and stacks are in the supcreritical range. In the range of supercritical Reynolds numbers from about $3 \times 10^{5}$ to $3.5 \times 10^{6}$ where the unsteady lift forces are random, the theory of random excitation [8] may be used to obtain estimates of the probable peak deflections. According to this theory, the mean-squared bending response $y(z)^{2}$ in a direction normal to the wind can be written as: (considering only the first bending mode of the structurc)

$$
\overline{y(z)^{2}}=\frac{1}{8} \frac{d}{U} \overline{C_{L}^{2}} F(S) \frac{W_{1}^{2} \phi_{1}(z)^{2}}{\omega_{1}^{3} M_{1}^{2}}
$$

where

$$
\begin{aligned}
W_{1} & =\int_{0}^{L} q(z) d(x) \phi_{1}(z) d z \\
M_{1} & =\int_{0}^{L} m(z)\left(\phi_{1}(z)\right)^{2} d z \\
m & =\text { mass per unit height } \\
q(z) & =\text { dynamic wind pressure per unit height } \\
& =1 / 2 \rho U(z)^{2} \\
d & =\text { diameter at height } z \\
L & =\text { total height of structure } \\
\phi_{1} & =\text { mode shape of first bending mode of } \\
& \text { vibration } \\
U(z) & =\text { velocity at height } z \\
\rho & =\text { Air density } \\
d & =\text { diameter at top of structure } \\
U & =\text { Wind speed at top of structure } \\
\beta= & \text { percentage of critical damping } \\
\omega_{1}= & \text { natural (circular) frequency of first bend- } \\
& \text { ing mode } \\
& =2 \pi f \\
f & =\text { frequency in cycles per second } \\
S= & \frac{f d}{U}=\text { Strouhal number } \\
F(S)= & \text { power spectrum of lift forces } \\
\overline{C_{L}}{ }^{2} & =\text { mean-squared lift coefficient }
\end{aligned}
$$

The power spectral term $\overline{C_{L}{ }^{2}} F(S)$ in Equation 6 is a measure of the mean-square lift forces on the structure. For cylindrical structures, Figure 3 shows a typical variation of $\overline{C_{L}^{2}} F(S)$ with Strouhal number $S$. This plot is based on experimental results obtained by Fung [4]. Fung measured the fluctuating lift forces 


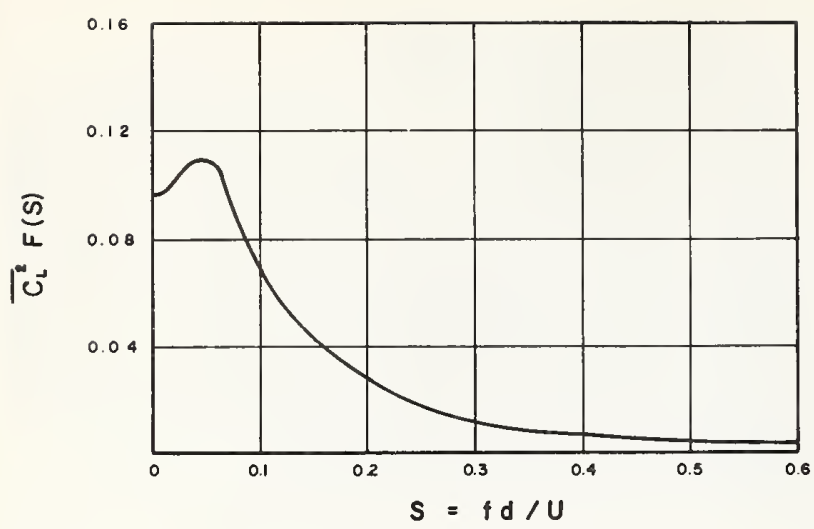

Figure 3. Fung's power spectrum for lift on a circular cylinder.

acting on a stationary circular cylinder for Reynolds numbers in the range of $0.3 \times 10^{6}$ to $1.4 \times 10^{6}$ and obtained its power spectrum as a function of Strouhal number by use of a wave analyzer.

It is seen from Figure 3 that $\overline{C_{L}^{2}} F(S)$ is a maximum at a Strouhal number of about 0.05 . With increasing Strouhal numbers the value of $\overline{C_{L}{ }^{2}} F(S)$ gradually falls to zero. The results in Figure 3 are normalized such that:

$$
\int_{0}^{\infty} F(S) d S=1.0
$$

Based on this equality, the mean-squared lift coefficient can be shown by integration to be $\overline{C_{L}{ }^{2}}=0.274$.

Nakagawa et al. [9] also determined the power spectrum of the fluctuating lift forces by measuring amplitudes of both oscillating circular cylinders in a wind tunnel and steel stacks in the field. The wind tunnel tests covered a Reynolds number range from 1.5 to $10^{5}$ to $1.5 \times 10^{6}$ and the field measurements covered a range from $2 \times 10^{6}$ to $8 \times 10^{6}$. Both Fung's and Nakagawa's wind tunnel data are in general agreement with the field measurements.

Figure 4 presents calculated deflections of the top of an actual 250 foot high, unlined steel stack (Stack $A$ ) which exhibited severe lateral oscillations at moderate wind speeds. (This stack has since been fitted with a spiral arrangement of vertical strakes to suppress the oscillations [19]. The efflux of the stack is 9 feet and the fundamental frequency, confirmed by field observation, is 1.16 cps. The Reynolds number, based on the efflux diameter, ranges from $0.8 \times 10^{6}$ for a wind speed of $15 \mathrm{fps}$ to $8.75 \times 10^{6}$ for a design speed of $160 \mathrm{fps}$. Estimates of the maximum deflections were obtained from Equation 6 using

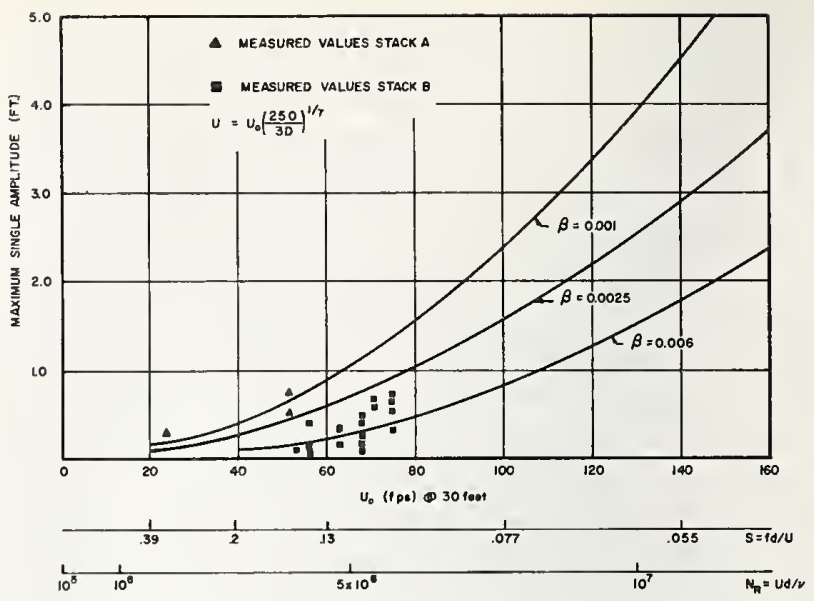

FIgURE 4. Variation of maximum amplitude of stack top versus wind speed and percentage of critical damping.

Fung's power spectrum. For calculating $U(z)$, it was assumed that the wind speed increased with height according to the $1 / 7$ power law:

$$
U(x)=U_{0}(z / 30)^{1 / 7}
$$

where $U_{0}$ is the wind speed at a reference height of 30 feet.

Estimates of the peak deflections $y(z) \max$ were obtained from the relation:

$$
y(z)_{\max }=3 \sqrt{y(z)^{2}}
$$

where the factor of 3 ( 3 times the standard deviation) corresponds to a probability of 0.9973 that $y(z)_{\max }$ will not be exceeded; i.e., during a storm, the value will not be exceeded, on the average, more than 0.27 percent of the time.

The calculated amplitudes pertain to assumed damping ratios, $\beta$, of $0.001,0.025$ and 0.006 . As expected, the amplitudes increase with an increase in wind speed and are greatest for the smallest damping ratio of 0.001 . For comparison, Figure 4 presents field measurements of the amplitudes for wind speeds up to $52 \mathrm{fps}$. These amplitudes are in good agreement with the theoretical results for a damping ratio of 0.001 , and suggest that the damping in the stack is quite low. Field data (Stack B, Reference 9) for a steel stack having similar dimensions and free vibration characteristics as Stack $A$, but having a damping ratio of .006 , are also brought into comparison in Figure 4. These data compare well with the theoretical curve for a damping ratio of 0.006 .

The experimental data of Figure 4 pertain to wind speeds at 30 feet in the range of $20 \mathrm{fps}$ to $75 \mathrm{fps}$. The Reynolds number, based on the efflux diameter and wind speed at the top of the stack, increases from 
$1.5 \times 10^{6}$ to $6 \times 10^{6}$ while the Strouhal number decreases from 0.35 to 0.10 . In the range of Reynolds numbers greater than $3.5 \times 10^{6}$, which corresponds to the periodic vortex shedding range, there is no change in the trend of the experimental data and the theoretical curves based on random excitation match the experimental data quite well. This is because, in this range of Reynolds numbers, the Strouhal number is much smaller than the Strouhal frequency at which vortices are shed (Figure 1) and a resonant is eliminated.

It is also noted from Figure 4 that there is no peak in the dynamic response at a Strouhal number of 0.2 , the value at which resonance is sometimes observed for cylindrical structures. In this case, a Strouhal number of 0.2 corresponds to a Reynolds number of $2.9 \times 10^{6}$, which is in the range where the vortex shedding is random. Power in this range is supplied over a wide range of Strouhal numbers and, as a result, excitation occurs over a wide range of frequencies.

Scruton [11] observed a similar phenomenon in a wind tunnel test and mentions that: "A model of the tower for the Museum Telephone Exchange, London, showed the effect of an aperiodic wake (random vortex shedding) for $3 \times 10^{5} \leq N_{R} \leq 3.5 \times 10^{6}$. This cylindrical structure was tested at values of $N_{R}$ up to $2.9 \times 10^{5}$. Oscillations were spasmodic and the amplitudes increased steadily as velocity was increased. There was no predominant frequency of vortex shedding, excitation occurring over a wide range of frequencies."

This phenomenon may seem inconsistent with the observed behavior of cylindrical towers and stacks which have exhibited peaked resonant vibrations at a Strouhal number of about 0.2 , and which appear to be relatively quiescent at other Strouhal numbers. However, apart from subcritical values of Reynolds numbers such peaked vibrations usually occur only when a Strouhal number of approximately 0.2 corresponds to Reynolds numbers greater than $3.5 \times 10^{6}$, where the vortex shedding is periodic. For instance, in 1953, a welded stack of $16 \mathrm{ft}$. diameter and natural frequency of $1 \mathrm{cps}$ came to resonance and failed in a wind of about $50 \mathrm{mph}$; the corresponding Reynolds number was $7.3 \times 10^{6}$ and the corresponding Strouhal number was 0.22 . Similar resonant oscillations at a Reynolds number of about $4 \times 10^{6}$ were also observed in recent wind tunnel tests at Langley Research Center, NASA, involving Saturn $1 \mathrm{~B}$ and Saturn V launch vehicles [10]. On both models, resonance occurred at a Strouhal number of about 0.2 .
In connection with the foregoing, it is apparent that an increase in natural frequency of a structure may not be a feasible means of reducing Karnan vortex oscillations, and, in fact, can be detrimental. This is because an increase in natural frequency is accompanied by an increase in the critical wind speed; if this critical wind speed corresponds to a Reynolds number in the range where the vortex shedding is periodie, a resonance condition may be created. However, an increase in natural frequency is advantageous when the critical wind speed is increased to a value greater than the probable maximum speed for the site.

\subsection{Sclf Excited Response}

For supercritical values of Reynolds numbers $N_{R} \geq 3.5 \times 10^{6}$, the vortex shedding is periodic and there is evidence $[10,11]$ which indicates that the associated aerodynamic forces are strongly dependent upon the motion of the structure. As mentioned above, instability tends to occur at a Strouhal number in the neighborhood of 0.2 , which happens to be the Strouhal number of the Karman vortex street at subcritical Reynolds numbers.

Although a Strouhal number of 0.2 should in general be avoided ior $N_{R} \geq 3.5 \times 10^{6}$, estimates of the vortex oscillations for cylindrical structures may, in this case, be computed using an approach advanced by Scruton [11].

In this approach it is assumed that oscillations will be maintained at constant amplitude $\eta$ when the energy dissipated by positive damping is equal to the negative aerodynamic damping provided by the wind stream; that is when:

$$
\left(\delta_{a}+\delta_{s}\right) \eta=0
$$

where $\delta_{a}$ is the logarithmic decrement of the negative, aerodynamic damping and $\delta_{s}$ is the logarithmic decrement of the positive, structural damping.

For a flexible structure, the negative acrodynamic damping can be determined by the integration:

$\delta_{a}=\int_{0}^{L} \rho d^{2} K_{a} \phi^{2}(z) d z / 2 \int_{0}^{L} m(z) \phi^{2}(z) d z$

where $\phi(z)$ represents the displacement mode of oscillation and $K_{a}$ is a non-dimensional parameter which must be determined from experiment. The value of $K_{a}$ is dependent upon the aerodynamic shape, the Strouhal number and the Reynolds number, and the amplitude of vibration. $K_{a}$ can be positive or negative and oscillations will be maintained only when it is negative and when the 
attendant value of $\delta_{a}$ is numerieally equal to the positive damping provided by the strueture.

For a subcritieal Reynolds number of about $1.2 \times 10^{5}$, Seruton determined the variation of $-K_{a}$ with displacement from experiments on smooth, eircular eylinders. Peak excitation oeeurred at a Strouhal frequeney of 0.2 and the variation of $-K_{a}$ with $\eta$ at this frequeney followed the hyperbolic relationship

$$
-K_{a} \frac{\eta}{d}=0.55
$$

Although Scruton's results were obtained at a subcritieal value of the Reynolds number, similar results were obtained by Reed [10] for supereritical Reynolds numbers in the periodic flow regime. These tests indicate that motion dependent forees, in the form of negative aerodynamie damping occur at a Strouhal frequeney of about 0.2 for $N_{R} \geq 3.5 \times 10^{6}$ and suggest that Seruton's results are also applieable to $N_{R}$ in this range.

Since $K_{a}$ and hence $\delta_{a}$ are dependent upon amplitude $\eta$, limiting amplitudes may be obtained by calculating values of $\delta_{a}$ for a number of values of $\eta$. The limiting amplitude (see Equation 10) is then determined by the interseetion of the $-\delta_{a}$ vs. $\eta$ and $\delta_{s}$ vs. $\eta$ curves. The structural damping, $\delta_{s}$, will, in general, depend upon amplitude, but insufficient information is available on this variation and $\delta_{s}$ is normally taken as a constant.

Measurements of $\delta_{s}$ made on stacks and towers in still ain have been reported by Seruton [11] and are found to range from about 0.02 to 0.18 , the damping being generally greater for conerete or eonerete-lined construetion than for unlined steel construction.

It is interesting to note that, on the basis of Equation 12, it is possible to relate the values of $K_{a}$ and $C_{L}$, the Karman lift coeffieient used by many writers to express aerodynamic lift forees $L$ as

$$
L=1 / 2 \rho U^{2} d C_{L} \sin \omega t
$$

Using the following equivalence [11]

$$
C_{L}=\frac{-4 \pi K_{a} \eta S}{d}
$$

with $S$ equal to the Strouhal number at resonance (i.e., $S=0.2$ ), then on substitution of Equation 12, $C_{L}=0.27$. This value is close to the mean-squared lift coefficient $\overline{C_{L}{ }^{2}}=0.274$ measured by Fung [4] for a stationary cylinder.

Fung tested up to $N_{R}=1.4 \times 10^{6}$ and found $C_{L}$ to be fairly constant for $N_{R}>4.5 \times 10^{5}$. With decreasing
Reynolds numbers below $4.5 \times 10^{5}$ there is suffieient experimental evidence which shows that $C_{L}$ increases to values between 0.6 and 1.0 ; this range of $N_{R}$, however, is not important for tall structures.

\subsection{Correction Procedures}

If ealeulations show that either exeessive amplitudes of Karman vortex vibration are to be expeeted or that small but sustained vibrations may oceur as a result of a eritieal wind speed equal to the prevailing wind speed for the area, several alternatives are available to the design. Sinee the aerodynamic stability of a strueture depends upon the aerodynamie shape, the frequency of oseillation and the structural damping, these alternatives inelude modifications to one or more of these factors. Modifying the structure height to diameter ratio or taper is usually the first approaeh to be made; but since this change may not be practical in many eases, alternate proeedures are available. These include:

1. Dynamic Spoilers

2. Utilization of bracing or guying

3. Utilization of frietional or impact damping deviees

4. Addition of an internal gunite or refraetory lining

5. Addition of mass or change in fundamental frequeney

In general, the addition of aerodynamie spoilers is often more feasible and more aeceptable than ehanges of the natural frequeneies or increase of the struetural damping. The purpose of spoilers is to "spoil" the airflow over a body in order to avoid the generation of vortices with a predominant frequency, or to break up the correlation of the aerodynamic exciting forces over its length.

An optimum eonfiguration of spcilers for cylindrical struetures was developed at the National Physical Laboratory [13]. It consists of a three start system of rectangular section strakes of height $0.09 d$ and of pitch $5 d$. In the case of circular towers and stacks, it is only necessary to apply this deviee to the top third of the structure.

Other spoiler configurations which have been found to be effeetive in reducing vortex vibrations of cylindrical struetures consist of a two or three start system of equally spaced vertical fins arranged in a helieal pattern [14]. The optimum height of these strakes is about $0.1 d$ and the optimum piteh is about 11d. This configuration has also been found to be effective when applied only to the top third of eireular eross section towers and stacks. 
Although spoilers are effeetive in redueing Karman vortex vibrations of cylindrical structures, they increase the drag loading. Wind tunnel tests [15] indicate that the drag eoeffieicnt for a cireular cylinder fitted with helieal strakes is about 1.15 based on the eireumseribing diameter. This clrag coefficient is approximately the same at that observed for smooth cireular cylinders at subcritical Reynolds number. A summary of reported drag eoeffieients for eireular cylinders is shown in Figure 5. In the supercritical Reynolds number range, the range of primary interest for the present problem, the drag eoefficient for a cylinder with spoilers is about twice as great as that for a smooth eylinder.

For adjacent structures sueh as staeks, stacks close to a strueture or small staeks, eorreetions can be made by bracing or guying. For tall flexible structures, guy wires would appear to offer a feasible solution provided there is adequate land space available for guy anchorages. In some eascs, guy wires have been used to eonnect dampers to the strueture $[20,21]$. For adjaeent staeks or towers near existing structures, bracing can be provided by inrarss of trusses or crossed pipes. In general, the spacing between adjaeent cylindrical structures should r.xecrol one and one-half diameters, which is the mininum distanee eited by Landweber [22] for out-of-phase vibration (lue to wind perpendicular to a line of eylindrieal structures; bracing can be expected to be effective only when the spaeing exceeds one and one-half diameters. Cross bracing is less effective in reclucing vortex vibrations for wind parallel to a line of cylindrieal structures, particularly if the spacing botween the structures is greater than two to threc diancters; sueh bracing, however, would appear to be effective in controlling slragwise vibrations due to buffeting in the wake of upwind struetures.

The utilization of frietional or impact type clamping devices usually have proven to be an effcetive means of eontrolling vortex vibrations of flexible, eylindrical structures. As noted above, the dampers arc usually eonnected to the strueture by means of guy wires. However, other types of damping deviees, which require no external conncetions, have been used or proposed. These devices eonsist of accelcration

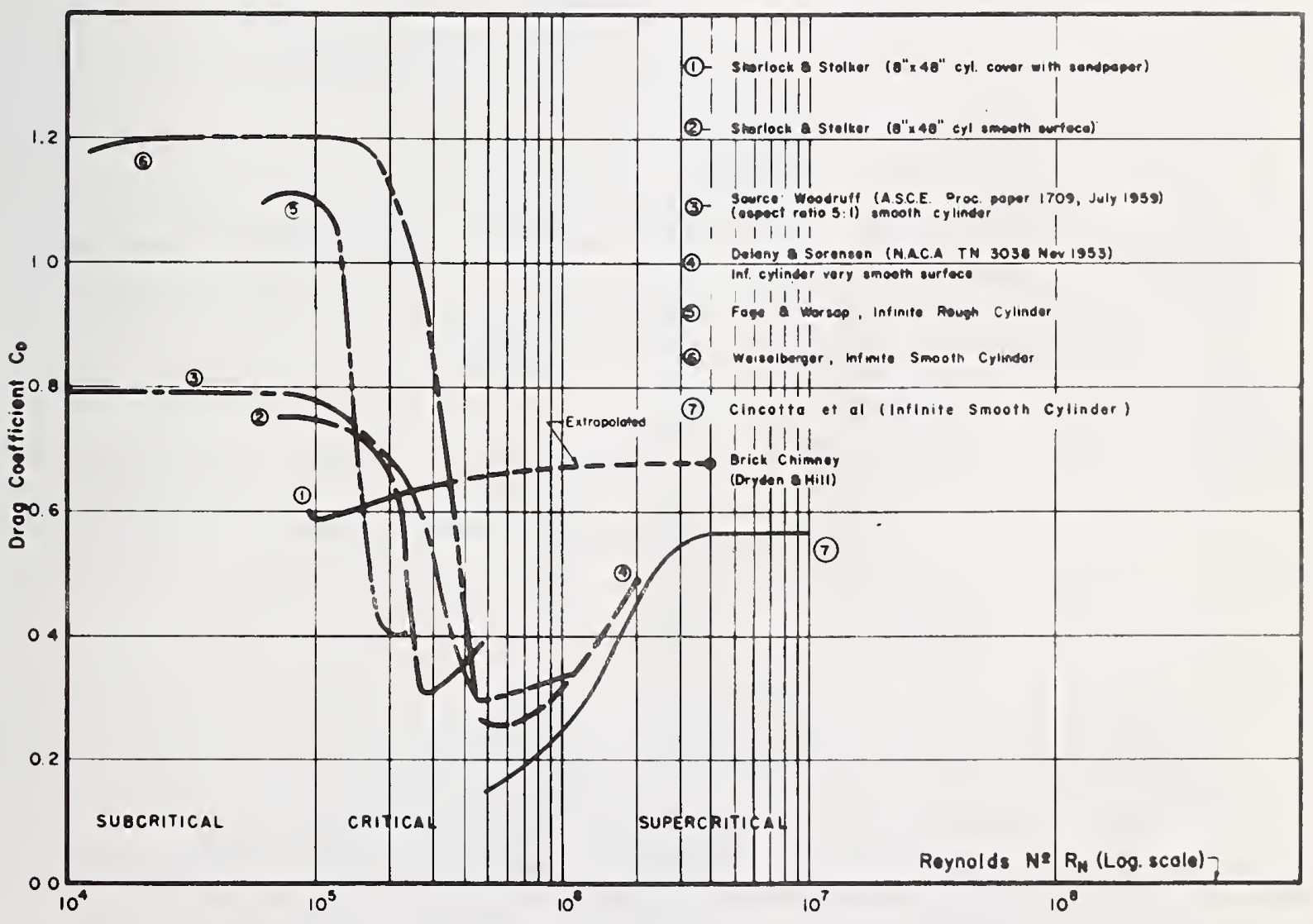

Figure 5. Drag coefficient versus Reynolds number. 
(impact) chain dampers [12] and auxiliary mass (Lancaster) dampers [10]. The acceleration chain damper, which provides beneficial damping when a vibrating structure causes hanging chains to impact against the walls of the structure, has been found to be successful in controlling vortex vibrations of a Wullenwebber antenna [23] and of an unfueled launch vehicle [12]. In general, both the impact and Lancaster type dampers become prohibitive for large structures because a relatively large mass for the damper is required to provide a substantial increase in damping.

In the case of stacks, a thick internal gunite or refractory lining greatly increases damping. The addition of a lining acts in two ways that can be approximated by calculation; first, mass is added to the stack thereby reducing its natural frequency; second, although refractory lining increases the moment of inertia of the stack which in turn increases the natural frequency, it reduces the dynamic deflection. The change in moment of inertia can only be expected to occur for a thick lining that is forced to act integrally with the stack.

A change in the natural frequency of a structure can, in some cases, be a feasible solution to the problem. For vibrations occurring within the supercritical range of Reynolds numbers, it is recalled that the vibrations are random when a critical Strouhal frequency of about 0.2 corresponds to a Reynolds number in the range, $3 \times 10^{5} \leq N_{R} \leq 3.5 \times 10^{6}$. On the other hand, when a Strouhal frequency of about 0.2 corresponds to a Reynolds number greater than about $3.5 \times 10^{6}$, a self-excited response phenomena will occur, and the amplitudes, depending upon damping, could result in a catastrophic failure. It is desirable, therefore, that the structure natural frequency and/or damping be such as to avoid the occurrence of this phenomenon.

In cases where it is impractical to increase the fundamental frequency of a strueture to such an extent that the critical wind speed for $S \approx 0.2$ and $V_{R} \geq 3.5 \times 10^{6}$ is raised above the maximum wind speed likely to be encountered, then consideration should be given to a reduction in frequency. A decrease in frequency serves to reduce the wind speed for vibration instability with the result that the energy input due to aerodynamic excitation is lessened. In some cases, a reduction in frequency would place a Strouhal number of 0.2 in the range of Reynolds numbers where the vortex shedding is random, thereby eliminating the possibility of a self-excited response; however, this reduction would tend to augment the random vibrations, although this may not be important provided there is a reasonable amount of damping available.

\section{Dynamic Response to Gust Loading}

\subsection{General}

In addition to the effects of lateral vibrations caused by Karman vortex shedding, it is necessary in the design of exposed structures to make allowances for the influence of dragwise vibrations due to gust loading. It is a comparatively well-known phenomenon that the turbulence characteristics of natural winds are distrubuted randomly in both time and space and that this turbulence produces a stochastic dynamic response of a structure. For such cases, it is meaningless to attempt a deterministic solution to the problem; however, power spectral techniques have proved to be powerful tools for analyzing the response of structures to random inputs and will be the approach discussed in the remainder of this paper.

Three elements are involved in determining the dynamic response (deflection, stress, etc.) of a structure to random wind loads: (1) the input power spectrum, defined by the power spectral density of atmospheric turbulence, $\Phi_{I}(f)$; (2) the frequency response function of the structure $T(f)$, defined in terms of the steady-state-load-response amplitude for a unit sinusoidal gust as a function of the forcing frequency $f$; and (3) the output power spectrum $\Phi_{0}(f)$ defined in terms of the response quantity of interest. The basic relationship between input and output spectra for linear systems is given by the expression:

$$
\Phi_{0}(f)=|T(f)|^{2} \Phi_{I}(f)
$$

where $|T(f)|^{2}$ is the square of the modulus of the structure frequency response function. The variance or mean-square response is obtained by integrating with respect to frequency; i.e.,

$$
\overline{\sigma_{R}^{2}}=\int_{0}^{\infty} \Phi_{0}(f) d f
$$

Under certain simplifying assumptions that the atmospheric turbulence is locally stationary and Gaussian, and the shape of the input power spectrum is invariant with the level of intensity of turbulence, it is possible to obtain quantities for the probable peak values of the dynamic response. For example, Davenport [24] has shown that the ratio, $g$, between the probable peak response and the RMS response $\left(\sqrt{\overrightarrow{\sigma_{r}^{2}}}\right)$ is given by: 


$$
g=\sqrt{2 \ln \nu T}+0.57 / \sqrt{2 \ln \nu T}
$$

where $T$ is the averaging period for the mcan wind (usually 1 hour) and where, according to Rice [25]

$$
\nu=\left\{\int_{0}^{\infty} f^{2} \phi_{0}(f) d f / \int_{0}^{\infty} \phi_{0}(f) d f\right\}^{1 / 2}
$$

The product $\nu T$ in a sense is a measure of the effective number of cycles of vibration that the structure will undergo during the averaging period $T$. The larger the number of cycles, the larger is the chance of an extremely large response occurring. The value of $g$ generally lies in the range of 3.0 to 5.0 .

\subsection{Application to Tall, Slender Structures}

Tall structures, such as towers, can be regarded as line-like systems, i.e., structures extended in only one direction. Consequently, all parameters of the system such as mass, stiffness, deflcction, etc., can be regarded as functions of only one spacc variable. On the basis of this assumption, the system will obey, in the linear elastic range, the partial differential equation

$m(z) \frac{\partial^{2} y(z)}{\partial t^{2}}+b(z) \frac{\partial y(z)}{\partial t}-c(x) \frac{\partial^{4} y(z)}{\partial z^{4}}=P(z, t)$

where

$z=$ the space variable measured from the ground

$t=$ the time variable

$y=$ the displacement of the structure

$m=$ the mass/unit length of the structure

$b=$ the damping/unit length of the

structure

$c=$ the bending stiffness/unit length of the structure

$P(z, t)=$ the applied load

The load $P(z, t)$ can be written in the form

$$
P(z, t)=\bar{P}(z)+p(z, t),
$$

where $P(z)$ is the mean load and $p(z, t)$ is the fluctuating component. Since the structure is linear, then the total response can be obtained by the superposition of the response due to $\bar{P}(z)$ and that duc to $p(z, t)$. The former can be obtained by the traditional methods of statics whereas the latter requires dynamic analyses and the use of statistical theories.

When normal mode analysis is applied to Equation 18 it can be shown [26] that the variance or mean- square deflection of the structure to the fluctuating load is given by:

$$
\begin{aligned}
\overline{y(z)^{2}}= & \frac{1}{32 \pi^{5}} \sum_{n} \sum_{m} \frac{\phi_{n}(z) \phi_{m}(z)}{E_{n} E_{m} f_{n}^{2} f_{m}^{2}} \\
& \cdot \int_{0}^{\infty} H_{n} *(f) H_{m}(f) C_{n m}(f) d f
\end{aligned}
$$

where

$C_{n m}(f)=\int_{0}^{L} \int_{0}^{L} \frac{\phi_{n}(z) \phi_{m}\left(z^{\prime}\right)}{c(z) c\left(z^{\prime}\right)} Y\left(z, z^{\prime}, f\right) d z d z^{\prime}$

$\phi_{n}(z), \phi_{m}(z)$ are the $n^{\text {th }}$ and $m^{\text {th }}$ mode shapes

$H_{n}(f)$ is the complex frequency response of the $n^{\text {th }}$ mode

$H_{n}{ }^{*}(f)$ is the complex conjugate of $H_{n}(f)$

$Y\left(z, z^{\prime}, t\right)$ is the cross spectrum of $p(z, t)$

and $E_{n}$ is the normalization constant of the $n^{\text {th }}$ mode where

$$
E_{n}=\int_{0}^{L} \frac{m(z)}{c(z)} \phi_{n}{ }^{2}(z) d z
$$

All of the necessary properties of the random wind loading are contained in the term $C_{n m}(f)$ and the solution of the problem to gust loading is reduced to the determination of this term in a form useful for design purposes. Unfortunately, $C_{n m}(f)$ depends upon $\phi(z), c(z)$ and the distribution of area and drag for the structure, and hence any given $C_{n m}(f)$ is specific to the particular structure whose modes and stiffness functions are used to evaluate the integrals.

If onc assumes that normal components of the exciting force are in the form $e^{i w t}$ then one can easily prove that the complex frequency response in the $n^{\text {th }}$ mode is

$$
H_{n}(f)=\frac{1}{1-\left(\frac{f}{f_{n}}\right)^{2}+2 i \beta\left(\frac{f}{f_{n}}\right)}
$$

where $\beta$ is the percentage of critical damping.

The normal mode analysis thus transforms the problem into a set of singlc-degree-of-freedom systems and the total response is obtained by a superposition of the individual modal responses. The amplitude of $H_{n}(f)$ represents the magnification factor, which is defined as the ratio of the deflection of the systcm during vibration to that corresponding to a static condition, and is given by 


$$
\left|H_{n}(f)\right|=\frac{1}{\sqrt{\left[1-\left(\frac{f}{f_{n}}\right)^{2}\right]^{2}+4 \beta^{2}\left(\frac{f}{f_{n}}\right)^{2}}}
$$

This expression tends, as would be expected, to the value of unity when $f$ approaches zero and approaches zero at large values of $f$. At the natural frequency, the response is maximum and the peak becomes greater as the critical damping ratio $\beta$ becomes smaller.

For most structures, the damping is small, and in this case only the diagonal terms in the summation (Equation 20) need be considered, i.e.,

$\overline{y(z)^{2}}=\frac{1}{32 \pi^{5}} \sum_{n} \frac{\phi_{n}{ }^{2}(z)}{E_{n}{ }^{2} f_{n}^{4}} \int_{0}^{\infty}\left|H_{n}(f)\right|^{2} C_{n n}(f) d f$

where $\left|H_{n}(f)\right|$ is given by Equation 24

and $C_{n n}(f)$ is given by Equation 21

with $\phi_{m}\left(\mathrm{z}^{\prime}\right)=\phi_{n}\left(\mathrm{z}^{\prime}\right)$

\subsection{Evaluation of $C_{n m}(f)$}

In addition to the modal, stiffness and basic drag data for the structure, certain statistical properties of the wind velocity are required for the evaluation of the integral given by Equation 21. The properties include the power spectrum of the wind velocity and its spatial correlation. The former reflects the distribution of energy among various wave frequencies, and the latter indicates how two velocity components located at two different points in space are correlated.

A spectrum of the horizontal velocity measured by Van Der Hoven [27] is shown in Figure 6. The curves are seen to be characterized by several prominent peaks. The region on the high frequency end, commonly referred to as the gust region, reflects the power

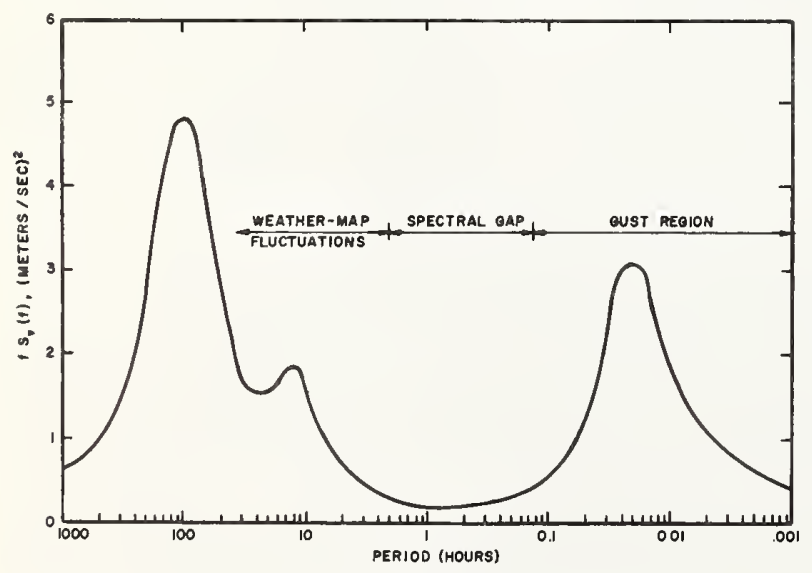

Figure 6. Van Der Hoven's gust spectrum. spectrum of turbulence for strong winds. If the spectrum, $S_{V}(f)$, of this region is plotted versus the frequency, $f$, on a $\log -\log$ scale, then the slope at the high frequency end of the scale would be close to the universal value in isotropic turbulence of $-5 / 3$. It should be mentioned that experimental results are available only up to a frequency of $3 \mathrm{cps}$. In accordance with the theory of turbulence, however, the spectrum contains very little energy at higher frequencies.

A region of very low energy exists to the left of the gust region and is usually referred to as the spectral gap. This gap is very helpful in the measurcment of wind, since it provides a means for obtaining meaningful and stable mean velocity measurements. For intance, if a record of one hour duration is averaged, the small-scale fluctuations will be averaged out and a true "mean" is obtained. To the left of the spectral gap, one finds large-scale fluctuations; these, however, reflect weather-map changes whose periods are much larger than the natural periods of vibration of structures and, hence, are of no concern to structural engineers. The spectral gap, which provides this clear-cut distinction between gusts and weather-map disturbances, may not exist, however, in certain extreme weather conditions such as tornadoes. These conditions are, nevertheless, of such rare and localized nature that they are normally considered as special cases by structural designers.

A generalized gust spectrum was obtained by Davenport [28] from 100 individual measurements made in strong winds at several locations all over the world and at heights in the range of 40 to $500 \mathrm{ft}$. This speetrum is plotted in Figure 7 , and ean be written in the form:

$$
S_{v}(f)=\frac{4 K \bar{U}_{00}^{2} x^{2}}{f\left(1+x^{2}\right)^{4 / 3}}
$$

where $S_{v}(f)$ is the spectrum of longitudinal velocity, $f$ is frequency in cps, $K$ is the surface drag coefficient, referred to $\bar{U}_{0}$ the mean wind speed at a reference height of 30 feet, and $x$ is defined by:

$$
x=4000 f / \bar{U}_{0}
$$

Harris [29] has shown that the Davenport spectrum dues not fully conform to certain known results of the equilibrium theory of turbulence, and proposed the alternate equation:

$$
S_{v}(f)=\frac{4 K \bar{U}_{0}^{2} x}{f\left(2+x^{2}\right)^{5 / 6}}
$$




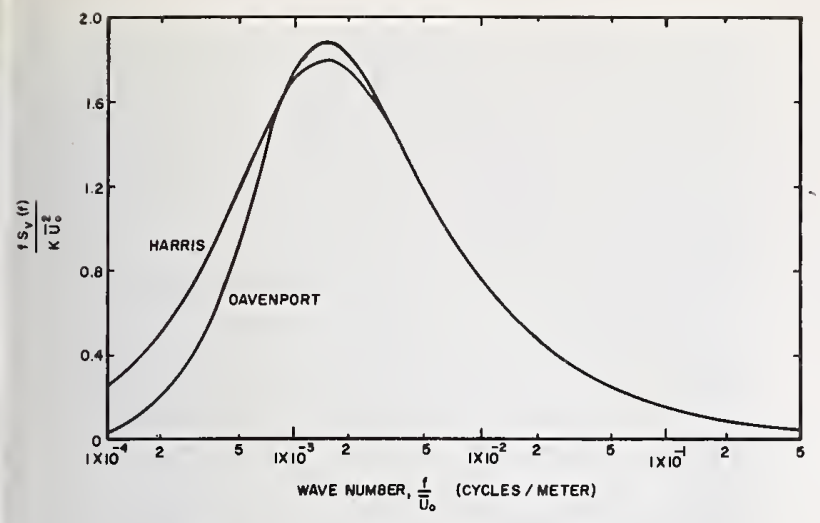

Figure 7. Spectrum of horizontal gustiness in high winds.

The two equations, however, are almost identieal except in the low frequency range, as can be seen in Figure 7.

The energy spectrum has been found to be independent of height in the height range indicated above ( 40 to $500 \mathrm{ft}$ ). Recent measurements by Harris [29] have also substantiated this assertion in the height range 58 to $598 \mathrm{ft}$. The speetrum, then, appears to be nearly universal in this height range and is only a function of the ground roughness, as refleeted by $K$, and the square of the mean wind speed at the referenee height. Average values of $K$ are given in Table 1 . These are seen to range from a value of 0.085 for large cities and very rough, hilly terrain, to a value of 0.005 for flat, open eountry. Interpolations of these values may be used for conditions intermediate to any of these exposures.

TABLE I. Surface drag coefficients, $K$ and velocity exponents, $\alpha$

\begin{tabular}{l|l|c|c}
\hline Exposure & Description & $K$ & $\alpha$ \\
\hline \hline Large cities and very rough, \\
$\begin{array}{c}\text { hilly terrain. } \\
\text { Rough wooded country, towns, } \\
\text { city outskirts. } \\
\text { Flat open country, open flat } \\
\text { coastal belts and grassland. }\end{array}$ & .0005 & $1 / 3$ \\
\hline
\end{tabular}

The other parameter whieh is needed in deseribing random wind loads is the eross-covarianee (or cross-eorrelation) funetion. This function measures the eorrelation between velocity fluetuations of a given frequeney located at two separate points in space. The process is analogous to that of the random eleetrieal signal in communieation theory. The latter has been extensively investigated by Riee [25] and others.

Davenport [30] has shown that the normalized correlation eoeffieients for the longitudinal velocity can best be represented in the exponential form,

$$
R(\Delta z, f)=e^{-c f\left(\Delta z \bar{U}_{0}\right)}
$$

where $\Delta z$ is the separation distanee and $C$ is an empirieal eonstant. A typieal eorrelation eurve is shown in Figure 8. Based upon experimental data obtained by Singer [31], the following values for $C$ may be used:

$$
\begin{array}{ll}
\text { Along-wind direction: } & C=7.7 \\
\text { Cross-wind direetion: } & C=23 \\
\text { Vertical direetion: } & C=7.7
\end{array}
$$

These values of $C$ elearly indieate that eddies are elongated in the direction of wind. This phenomenon may be attributed to the presenee of the ground surface.

The correlation coefficients are normally complex quantities and are made up of two quantities: in-phase and quadrature eomponents. In other words, the best correlation between two points may not neeessarily oeeur at the same time. The parameter expressed by Equation 29 is then the modulus of this eomplex quantity and is also called the square root of the coherenee funetion. The phase is found to be small and is normally neglected.

In the evaluation of struetural response to wind loading, the load speetrum rather than the wind velocity spectrum is needed. The former, however, ean be obtained from the latter as follows:

The drag foree on the strueture, $P(z, t)$, can be written as

$$
P(z, t)=1 / 2 \rho C_{D} A U^{2}(z, t)
$$

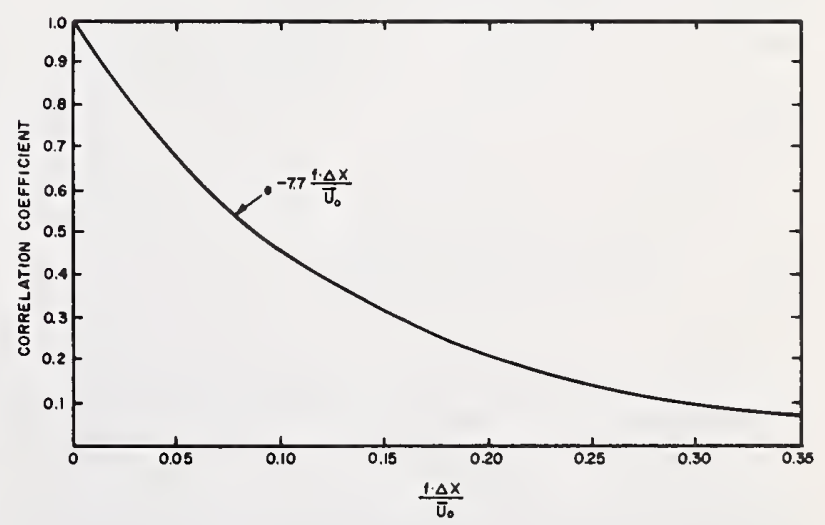

Figure 8. Typical correlation curve of wind velocity. 
where $\rho$ is mass density, $C_{D}$ is the drag coefficient, $A$ is the projected area and $U$ is the longitudinal component of the wind velocity. Writing $P(z, t)$ and $U(z, t)$ in terms of a mean and a fluctuating component, Equation 30 becomes

$$
\bar{P}+p=1 / 2 \rho C_{D} A(\bar{U}+u)^{2}
$$

where $\bar{P}$ and $\bar{U}$ are the mean components, and $p$ and $u$ are the fluctuating components. Neglecting the term $u^{2}$, Equation 31 yields

$$
p=\frac{2 \bar{P} u}{\bar{U}}
$$

Hence, the load spectrum, $S_{p}(f)$, can be written as

$$
S_{p}(f)=\frac{4 \bar{P}^{2} S_{v}(f)}{\bar{U}^{2}}
$$

where $S_{v}(f)$ is the velocity spectrum defined by Equations 26 or 28.

In the above derivation, it was assumed that the drag coefficient, $C_{D}$, is the static value corresponding to $\vec{U}$. Experimental results [30], however, indicate that this coefficient increases with frequency. For slender, flexible structures, the error derived from this assumption may be small, largely because of the fact that at large frequencies, i.e., gusts with small wavelength, the wind is poorly correlated.

In Equation 21, the term $Y\left(z, z^{\prime}, f\right)$ (the cross spectrum of the load) can be expressed as the product of the load spectrum $S_{P}(f)$ and the correlation function $R(\Delta z, f)$. However, for a three-dimensional structure, the spatial correlation in the three directions must be considered. It is reasonable to calculate the correlation between gusts over the flow region affecting the loading on the structure. This assumption is generally considered to be reasonable [32] and results in the following expression for spatial correlation

$R\left(z, z^{\prime}, f\right)=\left(\frac{2 \gamma-1+e^{-2 \gamma}}{2 \gamma^{2}}\right)\left(\frac{2 \mu-1+e^{-2 \mu}}{2 \mu^{2}}\right) e^{-2 \delta\left|z-z^{\prime}\right|}$

where

$$
\begin{aligned}
\gamma & =11.5 f \Delta x / \bar{U}_{0} \\
\mu & =3.85 f \Delta y / \bar{U}_{0} \\
\delta & =3.85 f / \bar{U}_{0}
\end{aligned}
$$

The first two products in Equation 34 were obtained by integrating the terms $e^{-2 \gamma}$ and $e^{-2 \mu}$ in the crosswind and downwind directions, over distances $\Delta x$ and $\Delta y$, respectively. For slender structures, it is implied in Reference 32 that $\Delta x$ be taken as the diameter of the structure and $\Delta y$ be taken as four times the diameter.
A limited amount of experimental data obtained by Vickery [33] indicates that the latter assumption may be unconservative and additional experimental data are needed to define the limits over which the correlation should be extended. It is generally considered to be conservative to assume that $\Delta y$ is at least equal to the diameter or downwind dimension of the structure.

If it is now assumed in the most general way that the mean drag loading $\bar{P}(z)$ varies with height, then it is easy to siow that $C_{n m}(f)$ can be written in the form

$$
\begin{array}{r}
C_{n m}(f)=4 S_{v}(f) \int_{0}^{L} \int_{0}^{L} \frac{\phi_{n}(z) \phi_{m}\left(z^{\prime}\right)}{C(z) C\left(z^{\prime}\right)} \frac{\bar{P}(z) \bar{P}\left(z^{\prime}\right)}{\bar{U}(z) \bar{U}\left(z^{\prime}\right)} \\
\cdot R\left(z, z^{\prime}, f\right) d z d z^{\prime}
\end{array}
$$

Where $S_{v}(f)$ is the velocity power spectrum given by Equation 26 or $28, R\left(z, z^{\prime}, f\right)$ is the spatial correlation function given by Equation 34 , and $\bar{P}(z)$ and $\bar{U}(z)$ are the mean wind load and wind speed at height $z$.

All of the significant properties of the fluctuating drag loading are contained in Equation 35. Hence, once the integration indicated by this equation is performed, the double summation indicated in Equation 20 or 25 is performed, followed by integration with respect to frequency, to obtain the variance of the deflection at any point in the structure. The variance of other structural quantities of importance such as shearing forces and bending moments, which are linearly related to displacement, may then be obtained using conventional procedures. The equations can be solved straight-forwardly by numerical integration with a digital computer.

It is generally assumed for gust loading calculations that $\bar{P}(z)$ and $\bar{C}(z)$ can be taken as hourly averages. When wind speed data for shorter averaging periods are available, the plot of Figure 9 may be used to convert these speeds to hourly averages. This plot was obtained by Durst [34] by means of a statistical analysis of data taken in strong winds over flat, unobstructed terrain. In Figure 9, $F$ represents the ratio of probable maximum wind speed averaged over time $t$ to the mean hourly' speed. For "fastest mile" wind speeds the averaging period $t$ is given by $t=3600 / \vec{V}_{f}$ where $t$ is in seconds and $\bar{V}_{f}$ is the fastest mile speed in miles per hour.

The variation of $\bar{U}(z)$ with height is generally assumed to follow the power law,

$$
\vec{U}(z)=\bar{U}_{0}(z / 30)^{\alpha}
$$

where $\bar{U}(z)$ is the velocity at height $z$ above the ground, $\bar{C}_{0}$ is a reference velocity at a height of 


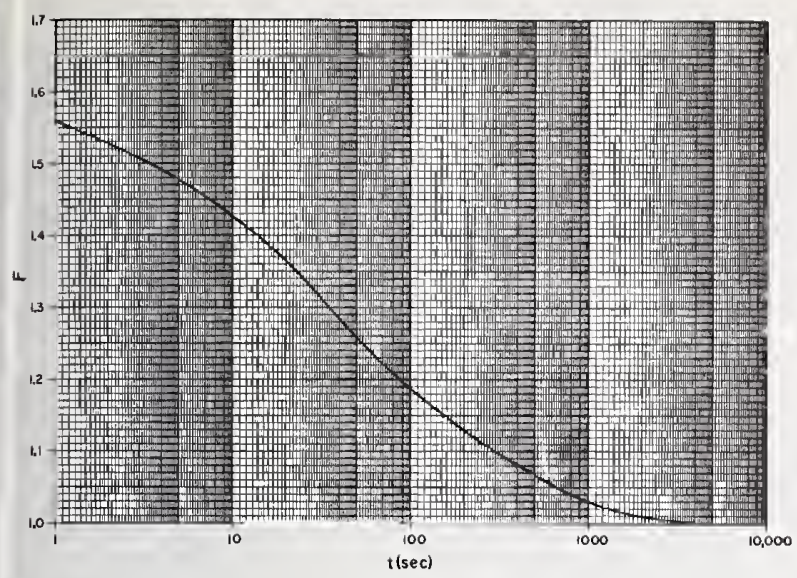

Figure 9. Ratio (F) of probable maximum speed averaged over period $t$ to that averaged over 1 hour.

usually $30 \mathrm{ft}$ and $\alpha$ is an exponent whieh depends on the roughness of the terrain. Average values of $\alpha$ are given in Table 1.

Using the eoneept of gradient height, i.e., the height at whieh the effect of ground frietion becomes negligible, hourly wind speed data for a given exposure may be converted to exposures of different roughness. Average gradient heights are in the order of $1,500,1,300$, and $900 \mathrm{ft}$ for exposures denoted in Table 1 by $A, B$, and $C$, respectively. Based on a 900 -ft gradient height for an open exposure $(C)$, the mean hourly speed in Exposures $A$ and $B$ may be related to that in Exposure $C$ by

$$
\bar{U}(z)=1.63 \bar{U}_{0, c}\left(\frac{z}{z_{\theta}}\right)^{\alpha}
$$

where $\bar{U}(z)$ is the mean hourly speed at height $z, \bar{U}_{o, c}$ is the hourly speed at $30 \mathrm{ft}$ for Exposure $C$, and $z_{q}$ is the gradient height. Equation 36 is plotted in Figure 10 for the three types of terrain.

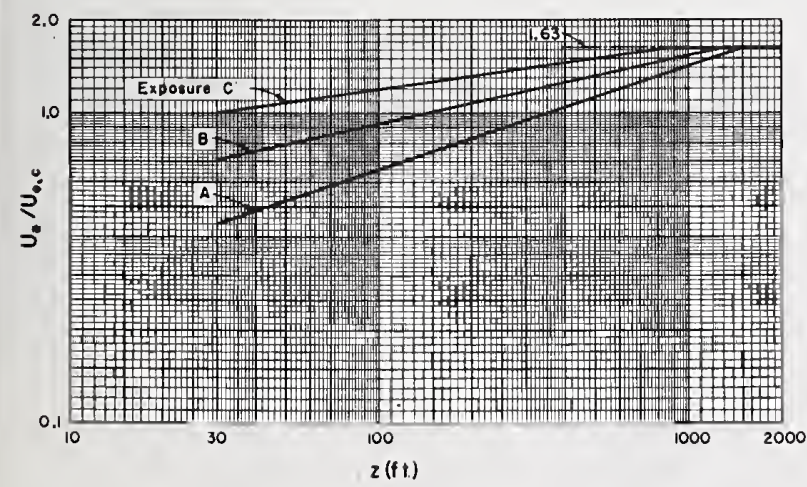

Figure 10. Wind velocity profiles.
It must be eautioned here that the above values of $\alpha$ and $z_{u}$ represent only average conditions, and do not apply in cases where extreme eonditions of shielding or ehanneling prevail.

\section{References}

[1] Von Karman and T. \& II. Rabach, Uber den Meehanismus des Flusigkeits und Luftwiderstandes, Phys. Zeits 13(2), 1912.

[2] Milne-Thompson, L. M., Theoretical Hydrodynamics, Macmillan, New York, N.Y., 1955.

[3] Roshko, A., On the Development of Turbulent Wakes From Vortex Streets, NACA Tech. Notes 2913, 1953.

[4] Fung, Y. C., Fluetuating Lift and Drag Acting on a Circular Cylinder in a Flow at Supercritical Reynolds Number, Shock and Vibration Bulletin Part II, No. 26, Office of Sccretary of Defense, December 1958.

[5] Cincotta, Joseph J. et al., Experimental Investigation of Wind Induced Oscillation Effects on Cylinders in Two Dimensional Flow at High Reynolds Numbers, paper presented at Meeting on Ground Wind Load Problems in Relation to Launch Velıicles, NASA Langley Research Center, June 7-8, 1966

[6] Roshko, A., Experiments on the Flow Past a Circular Cylinder at Very High Reynolds Numbers, $J$. Fluid Mechanics, 10, (3), 1961.

[7] Scruton, C., On the Wind-Excited Oscillations of Stacks, Towers and Masts, paper presented at Symposium on Wind Effects on Buildings and Structures, National Physical Laboratory, Teddington, Middlesex, England, June 1963.

[8] Hurty, Walter C. and Moshe F. Rubenstein, Dynamics of Structures, Prentice Hall, New Jersey, 1964.

[9] Nakagawa, Kenji et al., An Experimental Study of Acrodynamic Deviees for Reducing Wind-Induced Oscillatory Tendencies of Stacks, paper presented at Symposium on Wind Effects on Buildings and Structures, National Physical Laboratory, Teddington, Middlesex, England, June 1963.

[10] Reed, Wilmer H. III, Models for Obtaining Lffects of Ground Winds on Spacc Vehicles Erected on the Launch Pad, paper presented at Conference on The Role of Simulation in Space Technology, Virginia Polytechnic Institute Engineering Extension Series Circular No. 4, August 1964.

[11] Scruton C. et al., Wind Excited Oscillations of Structures, The Institution of Civil Engineers, Paper No. 6758, London, 1964.

[12] Reed, Wilmer, H. III, Hanging Chain Impact Dampers, paper prescnted at the International Research Seminar: Wind Effects on Buildings and Structures, Ottawa, Canada, Sept. 11-15, 1967.

[13] Scruton, C., Note on a Device for the Suppression of the Vortex Fxcited Oscillations of Flexible Structures of Circular or Near Circular Cross Section, With Special Refcrenee to its Application to Tall Stacks, National Physical Laboratory Aero. Note 1012, April 1963.

[14] Goldman, R. L., Karman Vortex Forces on the Vanguard Rocket, Shock and Vibration Bulletin Part II, No. 26, Office of the Secretary of Defense, December 1958. 
[15] Cowdrey, C. F. and J. A. Lawes, Drag Measurements at High Reynolds Numbers of a Circular Cylinder Fitted with Three Helical Strakes, National Physical Laboratory, Aero Report 384, July 1959.

[16] Steinman, D. B., Problems of Acro-Dynamic and Hydrodynamic Stability, Proc. Third Hydraulies Conf. Univ. Iowa Studies in Engin. Bull. 31, 1946.

[17] Weaver, William Jr., Criteria for Design Against Wind Induced Vibrations in Antenna Members, Annals of the New York Academy of Sciences, Vol. 116, Art. 1, June 26, 1964.

[18] Guyton B. et al., Maximum Amplitude of Oscillation of a Cylinder due to its vortex wake, Proc. ASCE, Vol, 82 No. HY4, August 1956.

[19] Ammann and Whitney, Investigation of Karman Vortex Shedding of Stcel Stacks, Prepared for Chemical Construction Company, New York, August 1968.

[20] Boorne, R. A., The Aeroelastics of Tall Stacks, Inst. Aero. Sci., Preprint No. 851, October 1958.

[21] Den Hartog, J. P., Recent Technical Manifestations of von Karman's Vortex Wake, Proc. Nat. Acad. Sci. of USA, Vol. 40, 1954.

[22] Landweber, L., Flow about a Pair of Adjacent Parallel Cylinders Normal to the Stream, David Taylor Model Basin Report 485, 1942.

[23] Farmer, Moses, G. and Reed, Wilmer H. III, Study of Wind Excited Oscillations of High Band Wullenwebber Antenna, NASA LWP-324, November 1966.

[24] Davenport, A. G., The Application of Statistical Concepts to the Wind Loading of Structures, Proc. Inst. Civ. Eng. $19,449-472,1961$.

[25] Rice, S. O., Nathematical Analysis of Random Noise, Bell System Tech. Jour, 23, 282-296, 1944 and 24, $46-59,1945$.

[26] Thompson, W. T. \& Barton, M. V., The Response of Mechanical Systems to Random Excitation, J.Appl. Mechs. Vol. 24, No. 2, 1957.

[27] Van der Hoven, I., Power Spectrum of Horizontal Wind Speed in the Frequency Range from 0.0007 to 900 Cycles per Hour, J. Meteor. 14, 160-164, 1957.

[28] Davenport, A. G., The Spectrum of Horizontal Gustiness Near the Ground in High Winds, Quart.J. Roy. Meteor. Soc. 87, 194-211, 1962.

[29] Harris, R. I., Measurements of Wind Structure at Heigh ts up to 598 Feet Above Ground Level, Electr. Res. Assoc. Report No. SPI/T31, 1968. (Unpublished).

[30] Davenport, A. G., The Buffeting of Large Superficial Structures by Atmospheric Turbulence, Annals of the N.Y. Academy of Sciences, Vol. 116, Art. 1, 1964.

[31] Singer, I. A., Wind Gust Spectra, Annals of the N.Y. Academy of Sciences, Vol. 116, Art. 1, 1964.

[32] Vellozzi, J. and Cohen, E., Gust Response Factors, Proc. ASCE Vol. 94, No. ST6, June 1968.

[33] Vickery, B. J., On the Reliability of Gust Loading Factors, Paper presented at Tech. Meeting Concerning Wind Loads on Bldgs. and Structures, Nat. Bur. of Stds., Jan. 1969.

[34] Durst, C. S., Wind Speeds Over Short Periods of Time, Meteor. Magazine, Vol. 89, 1960. 


\title{
SOME DEFICIENCIES IN CURRENT METHODS OF ANALYSIS
}

\author{
R. W. Clough, Chairman \\ Division of Structural Engineering and Structural Mechanics \\ The University of California \\ Berkeley, California 54720
}

\begin{abstract}
Deficiencics in methods of analysis for structures subjected to wind forces are considered. Areas of similarity between wind and seismic forces and their treatment by current building codes are discussed. Deficiencies include a neglect of the dynamic nature of the wind problem, a lack of basic information, and an inadequate definition of acceptable levels of risk.
\end{abstract}

Key words: Buildings; climatology; seismic loads; structural engineering; wind loads; vibrations.

My talk will give us an opportunity to try to get back on schedule, because I don't have a formal paper, and I don't intend to take anything like the allotted time.

As was mentioned, my subject is supposed to be dealing with the deficiencies in the wind load problem. I am afraid the principal deficiency that will show up is my own lack of experience with this problem! My experience is highly limited in the wind area. What I want to do is to talk very briefly about a specific problem that I was associated with. And in connection with this you might keep in mind that my point of view in working with this problem was as a structural dynamicist working primarily with earthquake engineering-type problems.

The particular problem that I was confronted with was the analysis of a guyed tower. This was a tower which was to be a rather monumental structure. It was supposed to support a restaurant at a very high elevation, and the analysis seemed to pose a considerable challenge as far as my own field of interest was concerned. At that particular time, we happened to be working on analysis techniques for nonlinear structures at Berkeley, and this seemed to be a good opportunity to apply some of our computer programs to a very practical example. After we got into the analysis of the problem, it turned out that the principal question of concern to the design engineer was the dynamic bchavior of this structure, which, of course, was quite a change of direction as far as our analytical requirements were concerned. But, inasmuch as we had had quite a bit of experience with earthquake design analyses for dynamic effects, we felt that we probably could divert our direction, here also, into the dynamic direction, so we proceeded to set up a computer program for the dynamic analysis of this non-linear structure.

The program was based on a two-phased operation. We started by considcring the mean static wind load acting on the structure and treated this as an iterative solution of the nonlincar problem, successively changing the stiffness properties of all the structural components in accordance with the forces which they were carrying. After we got the nonlinear solution, we then proceeded with the dynamic analysis for which we assumed the structure would behave linearly, vibrating as a linear systcm about the non-linear equilibrium positions defined by the mean wind pressures. This approach seemed reasonable to us, and, of course, it was a fairly straightforward problem to calculate the vibrational properties for the particular stiffness coefficients associated with the mean wind condition which we had previously calculated. So we went through the standard vibration analysis, and then used mode superposition procedures to calculate the dynamic wind response.

During the time that this analysis was under way -in other words, while we were setting up the computer program to do the things which we thought needed to be done-I had my initiation into the deficiencics in this whole wind business. Of course, we needed information about the types of loadings to be applied to the particular structure at the particular location, and very quickly we found what we thought were adequate data for preliminary design purposes, describing the mean wind conditions which we should apply to the structure. But, when we began looking into the problem of dynamic behavior of the winds and dynamic variations of velocities or 
pressures, I was amazed to find that the Weather Bureau simply didn't make measurements of that type and had no statistics of any sort which were directly applicable to our problem. This, of course, completely limits the capability of an analyst with the kind of approach that we were proposing to take. Fortunately, after a number of inquiries, we were directed to the NASA group which has bcen making measurements at the Cape Kennedy tower, and they were kind enough to supply us with some excellent wind data for their 150-meter tower system which we felt we could distort sufficiently to serve the purposes of our own analysis. So, with this information, we were actually able to make a deterministic study of the behavior of this particular nonlinear structure. About the time we got the data, unfortunately, the whole project was canceled, and we never did carry out the complete set of analyses that we had in mind. We had just started making some comparisons of nondeterministic studies-following Davenport's techniques-to compare with our deterministic studies. Our original hope was to use our deterministic analysis as a substitute for wind tunnel studieswe thought we could actually introduce specific winds, study the actual behavior of the structure from a mathematical model of the structure, and, by this approach, get something equivalent to a wind tunnel study. Ultimately, of course, we recognized the need for the nondeterministic study, to get some estimate of the overall performance to be expected over a long period of time.

This is the experience that I had. In connection with this experience, I recognized a lot of deficiencies in the wind load picture, and I also recognized that there are very definite similarites between the deficiencies in the wind problems and those which we recognize in the earthquake engineering field. I thought I might, just very briefly, relate some of my concepts of these deficiencies.

The first deficiency, I think, is in the code specifications themselves. Both in the case of the earthquake problem and the wind problem, the code treats the behavior of the structure as a static problem. The dynamic nature of the problem which actually exists is essentially ignored by the code. In this respect, I think the earthquake business is a little bit ahead of the wind business, because, at least in the specification of the seismic coefficients, there is some recognition of the dynamic properties of the structure; the seismic coefficients arc presented as a function of the frequency of vibration of the structure in the typical code approach. Also, I think that it has become rather common practice now in these monumental-type structures for rather complcte dynamic analyses to be made of the seismic behavior of structures.

Very, very few really large, important structures in seismic regions are now designed without having rather thorough digital computer studics of their dynamic behavior under seismic conditions. In both of these respects the wind load deficiencies seem to be more severe than those in the earthquake area. There is very little direct recognition in the code of the dynamic nature of the problem, and I think only in a very few instances, such as the ones which we have heard about this morning, have we had significant analyses of the wind behavior, including some consideration of the dynamic response. Both of these factors - the improvement of the code, and considerably more actual study of the dynamic behavior of the structure in the analysis phase-are necessary.

The second area of similarity in deficiencies between the wind and the earthquake problems is in the lack of information. In the earthquake area, the reason for this shortage of information is quite obvious. The major earthquakes, which are the primary hazard to our structures on the West Coast, are very, very rare occurrences. The Coast and Geodetic Survey has a network of some 250 strongmotion seismographs distributed throughout the seismic regions; but, even with this very large network available, we have very, very few records of important earthquakes. In fact, we have no records of really major earthquakes of the Anchorage or Chile proportions. This, I think, is not so much a deficicney on the part of the people interested in making the measurements as it is a fact of life. We just don't get that kind of information very frequently. On the other hand, in the case of wind data, it seems that wind information is really quite available; it's just a matter of devising suitable instrumentation systems and going to work on getting the information. I think the NASA tower at Cape Kennedy is an excellent piece of work in this direction; and I think the NBS-ESSA cooperative program which is being set up here at this facility is, also, a very good step in that direction. But these are very limited efforts in what should be a very important area of investigation.

Now, in this regard I might point out that the City of Los Angeles in its most recent code adopted a requirement that any major structure must include two strong-motion seismographs in the structure. They put the burden of providing instrumentation on the owners of the buildings in this case, and I think there could be some consideration given in the wind 
loading area to the same type of thing. I think inajor buildings such as the United States Steel Building and the World Trade Center could very well be forced by some sort of code requirements to include in their actual cost of the structure some instrumentation which would benefit the whole profession.

Finally, the other area of similarity, in the deficiencies of the design problem, between earthquake and wind is in the area of defining the risk for which we are actually designing structures. Code provisions are established as rather sterile quantities, with very, very little indication of what kind of risk one is accepting-whether it's a hundred-year condition or a 10-year condition-and they also say nothing about the level of damage which might well be expected to be associated with that particular loading condition. In the case of the earthquake problem, the typical designing engineer's philosophy is that he wants his structure to perform well-that is, without structural damage - in relatively moderate earthquakes, but he is willing to accept a significant amount of structural damage in a major earthquake. Of course, he is hoping to avoid any total collapse and loss of life in a major earthquake, but the concept of accepting damage in a major earthquake is very well accepted throughout the engineering profession on the West Coast. Now, this is a nice philosophy of design; I think it's a consistent one in terms of the economy of the country, but, associated with that philosophy, there have been no indications of the kind of earthquake we are talking about when we specify our seismic coefficients-no indications as to whether these coefficients are supposed to relate to a 10-year earthquake or a hundred-year earthquake, or whether there should be any danage associated with the structure that is designed accorling to these coefficients. This is one of the tremendous deficiencies in the whole earthquake problem; there has been no real attention paid to the specification of these basic coefficients. What should be done is to establish the level of earthquake for which one intends to design and then establish some solt of recurrence date for which that earthquake would be expected, and have this clearly indicated in the code provisions. Apparently the same situation is also true with respect to the wind problem. There seems to be no indication of whether the 40-pound pressure which, apparently, is now specified in New York, is associated with a 10-year return period or a hundred-year return period, and apparently, there is no indication of whether the structure is expected to come through the design loading conditions without damage or whether some significant amount of damage is to be expected. It seems to me that we can't continually increase the factors of safety of our structures simply by increasing the load coefficients one way or another, without somewhere indicating the kind of risk which we associate with these various factors of safety. And, I think that very soon in both the carthquake and wind business, we are going to have to make some sort of direct attack on this problem of relating the code provisions to some measure of the risks which we are taking.

Those are the only comments which I have to make. I thank you very much. 


\title{
PANEL DISCUSSION
}

\author{
Mr. Edward Cohen, Chairman \\ Amman \& Whitney \\ New York, New York 10011
}

Dr. A. Allan Bates

Chief, Office of Engineering Standards Liaison

National Bureau of Standards

Washington, D.C. 20234

Professor A. G. Davenport

Faculty of Engineering Science

University of Western Ontario

London, Ontario, Canada

Mr. H. C. S. Thom

Senior Research Fellow

Environmental Data Service, ESSA

Silver Spring, Maryland 20910 


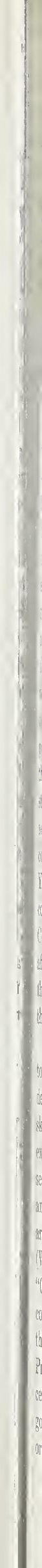




\section{PANEL DISCUSSION}

\section{Dr. BAtes:}

I am going to inject an unexpected note into this discussion-a notc which may sound slightly ominous to some of you; actually, it is not ominous, I assure you. Ed [Cohen] referred to the fact that there is now in existence an Office of Standards Policy (now office of Product Standards). This office, while it is located within the Department of Commerce, has rcsponsibilities which spread far beyond the Department of Commerce-spreading to all the agencies of the US Government, departmental, regulatory, and otherwise, which are concerned with standards and matters relating to standards, such as building codes, which are, of course, in large part, compendiums of standards. This newly established office is only one part of a general concern of which I am sure you are all aware to some degree. There is a concern in governmental circles-Federal, State, and otherwise-over the development of standards in this country under the traditional organizations, which have been largely private. Our standards and codes have evolved from privately introduced, privately conducted actions; and this has endowed the United States with one of the richest bodies of standards in the world. It has been a traditional activity which has served us well but one which has come under increasing criticism in recent years. You're all aware of the concern of the Congress over consumer standards. You're aware of the concern of Congress over standards in general, especially as they affect the public welfare. Now, if there is anything that affects the public welfare, it is the standards and the codes which relate to building and construction.

The two speakers yesterday were complementary to each other in a most useful way. Professor Maugh dealt in a very practical way with the bones and skeleton-and Wayne Koppes with the skin or exterior. Wayne Koppes showed us some slides-a series of pictures of damage, in which stecl spandrels and windows were torn loose by wind and went flying around the atmosphere, to possible public detriment. (Wayne's slides might collcctively have been entitled "Gone with the Wind!') The implication of public concern and public welfare was so great, not only in those slides, but in the remarks which he and Professor Maugh and others made, that it is quitc ccrtain that we are delving here in a field which is going to be looked into by Congress in the ncxt year or two. Professor Maugh pointed out, I think, the primary areas of ignorance. He related them to threc major aspects of our subject, and he started out by saying that we need a great deal more information, which is, I guess, pretty well known-although this is not to despair over getting it, nor is it to be derogatory of the vast amount of information we alrcady have. He related his remarks to frameworks in the proccss of construction, that is buildings in the process of erection, which are perhaps sometimes peculiarly vulnerable to damage by dynamic forces, and yet, in which case we have less information than we have for completed buildings. He also spoke of the necessity of relating the frequency and mode of response in buildings - in high-rise buildings - with relatively high frequencies, and related that to the subjective factor, that is to say, how much the average human being will stand without alarm, in building moition. This is certainly a subjective factor in the study - the kind which should be studied more often. He then went on to speak of structures with relatively low frequencies. This gets us into areas in which both potential danger and ignorance are, perhaps, becoming magnified. Incidentally, in Moscow, a year ago, I stood at the foot of a TV tower which is, according to my Russian informant, $1,700 \mathrm{ft}$ tall with a restaurant at the $900-\mathrm{ft}$ level, and he told me, if I understood him correctly, that the structure was expected to sway 3 meters in the wind at the top. Now, the frequency of that structurc is, of course, not very high, and one wouldn't get the feeling of vibration up there. But, this is the sort of thing we are getting into, and in which our ignorances are bccoming magnified as we become bolder in design.

Wayne Koppes pointed out that the frequency of danger and, indeed, the totality of danger - the total summation of danger and of damage--is not so much with the structure, but with the localized components of the skin, the windows, the spandrels, and so forth. He emphasized further that the negative pressures are, in general, apt to be higher than the positive pressures, and this is, likewisc, an area in whichwhile we arc not ignorant-we know much less than we should. He also spoke of the funncling effect, which is obviously very important and of high potential danger to the welfare of persons. He spoke of the instance in which, I think he said, persons wcre-or potentially were-blown into a pond; whilc the pond was shallow enough that the effects werc 
not too damaging, this illustrates what can happen due to these strange effects.

Both speakers repeatedly referred to building codes, and Professor Clough, this morning, returned to them most emphatically in emphasizing the deficiencies of codes. This brings me back to my opening remarks, that codes and standards have become of such concern to the public that the deficiencies which have been so emphasized here throughout yesterday and today, simply cannot be permitted to continue. And indeed, they are not continuing in the same order of magnitude as in the past. The deficiencies of coles and standards are, I think, quite certain to be the object of public attention-a good deal of public attention. I can refer you to a report, here, by a Congressional committee which eame out only a very few days ago, in which the recommendation is made that every voluntary standard, in effect, shall be subjected to a Governmental review, a very extensive three part Governmental review-one for the legitimacy of the procedure which has been followed; one for the technical content, the adequacy and accuracy of the technical content; and finally, the third review which relates to what we might call the economic and social effect of the standard. And, the recommendation is that all private voluntary standards be subjected to that review. Now, literally, this is almost an impossible task to carry out, and, if and when this comes to your attention, I would suggest you get not too greatly alarmed, as you might if you took the language literally-because an impossible task can be dealt with on the basis of its impossibility. However, that task can be modified to become possible, and will have a rather strong effect upon such organizations as are represented by this group here today, which are largely private-although not entirely so.

So my principal concern is the codes and standards as viewed by any and all Governmental agencies, regardless of their origin-private, Governmental, or otherwise - that these codes and standards shall be reviewed with due diligence as they relate to the public concern and the public welfare. Obviously, no building code can ever be a perfect building code. Wayne Koppes made this point-that the extremely complex local aspects of wind load and wind damage, positive and negative, are such that it is almost hopeless to ever reduce them literally to a complete code which will take all of the curious aspects of buildings into mind. So, it is not expected that building codes or building standards can be reduced to perfect coverage which will assure no possibility of damage or discomfort or danger within reasonable ecomonic limits; but it is expected that due diligence will be exerciscd-and I'm happy to say, as representative of this particular office which may have this task, that I am delighted with the diligence and the advancement in knowledge which has been shown by the speakers in the discussions of this session. I think it's been a most fruitful session, and I hope it will be followed by others, extending the vast reservoir of private competence and private knowledge which is represented here and in related organizations into sufficient and proper progress in building codes and standards.

\section{Professor Davenport:}

Mr. Chairman, I felt that probably my most useful task might be just to try to summarize some of the keynotes that have appeared in some of the valuable papers we've heard. I'll try to deal with them in not necessarily the sequence in which they were given, but rather in a manner more orderly in terms of the development here. In this instance, I would like to start with some comments on the meteorological area, which I feel is the basis of most of our technical problems.

We saw from Professor Gill's paper some cvidence that the instruments which we use to measure wind speeds are extremely important. And, there was a relationship-I felt--between his and some of the remarks which Mr. Fichtl and Mr. Kaufman made in their very interesting paper. One of the matters which concerns us is to try to find an adequate means for referencing wind speed on a national basis or on a continental basis, if you like. There are several approaches to this. The Weather Bureau and the Canadian Department of Transport have used what are fairly standard measurcments-they've used hourly wind speed; they've used maximum gusts and in the United States they have used the fastest mile. And, there are also some upper level wind aeronautical observations which are becoming cxtremely valuable. I feel that there is a very great need to try to communicate, to the meteorological organizations, the need for developing a consistent type of measurement. This point was referred to by $\mathrm{Mr}$. Smith. In the brief discussion this morning, I came out in favorfor technical reasons - of an average which is based on something like a 15 minute average which is justifiable both for structural and meteorological reasons.

There are several difficulties which exist at present. One is the determination of maximum gusts. As we saw from Professor Gill's paper, the type of instruments which are used affects the reading. If you use 
the fastest mile, you find that the estimate of the fastest mile from actual records is an extremely tenuous business when the wind speed gets very high, for the simple reason that you're trying to measure very small increments of distance on a chart of paper. There's never been any estimate that I've seen of the accuracy of the actual data that has been presented. The use of hourly averages is very much colored by the method of observation, and in not all cases has the hourly average been the true average, but has been, rather, a guess by the meteorologist who's on duty. These point to the fact that there is a need to improve accuracy in the records; and secondly, a need to develop some consistent estimate of wind speeds from which we can build a useful model of what the wind environment of the structure is likely to be. Sometimes I've felt that we have looked at the definition of the meteorological problen rather like the motorist in Nova Scotia who asked the way to somewhere from a local, and the local replied that, "If I wanted to get to there, I wouldn't go from here." I feel that to try to evaluate the meteorological problem from the vantage point of the surface of the globe is probably the worst possible starting point to get to the final objective.

I feel very strongly that the kinds of observations which we have tend to be misleadingly different - due to entirely local effects-if a town has grown up around the anemometer, then you're likely to see. that reflected in the velocity of the wind. Historically in the United States, with the coming of aviation, there was a tendency to move most of the first-order meteorological stations off to airports from their positions in the centers of cities. This itself dramatically increased the estimates of wind speeds, and hence the wind loads. This was entirely unrelated to a change in climate; it was simply that the instruments were moverl.

I feel that there's a tremendous amount of information which is available, both to structural engineers, and also to people who are interested in this kind of research. We've got the task ahead of us to unscramble the information that is available, and I think also, we have a task to improve the nature of the records which are currently being marle.

The value of upper-level winds is very useful in this respect. And, I've been most impressed by the ICAN-I don't know what that stands for, something about International Civil Aviation Network-these types of organizations are interested in winds which are free from the influence of the earth, and it's surprising how much order seems to emerge from the chaos which comes from relying on surface observations.
One factor which hasn't been touched on very much in the meteorological area is the importance of tornados. Mr. Thom might have some very useful observations in this connection. I think, though, that if you were to order the damage in Canada and the United States as to order of importance per year, I'm sure that tornados would head the list. I suspect that they cause more damage on an annual basis, than do earthquakes-and, probably, more deaths. Yet, we have extremely few handles by means of which we can get hold of this problem of tornado action.

The second area that I'd like to touch on is the question of aerodynamics. In Professor Cermak's paper, the value of wind tunnel techniques was noted. We also saw pictures of his micrometeorological wind tunnel at Colorado State University which was the first effective instrument on this continent which could handle structural problems in, what I consider to to be, an adequate way. There is a long history to wind tunnel testing procedures. The evolution has been, naturally, organized around the availability of wind tunnels. And, the kind of wind tunnel that developed for aeronautical research was a very logical one. It was designed, usually to be turbulencefree because the loads on aircraft are turbulence-free. It was designed to obviate entirely the kind of conditions which really exist at the level of the structures we're building. The micrometeorological or boundary layer wind tunnel creates a flow representative of the wind. I believe it has to be used more often, and the importance of its use - as distinct from other types of wind tunnels-has to be emphasized.

Martin Jensen, a very well-known worker in this area, once remarked that he felt that the aeronautical wind tunnel had already had nine lives in this kind of problem area-that its inadequacies have been demonstrated many times, but still people will continue to test in aeronautical wind tunnels, in spite of quite strong evidence that some of the problems are inadequately dealt with in this kind of environment.

We heard from Dr. Simon of the importance of vortex shedding, and this, again, is a highly interesting problem with which we have to grapple. His was the only contribution which dealt really effectively with this problem-but which was, perhaps, more associated with the non-turbulent situation. When we transfer our thinking to turbulent flow, our experience is that we find a lot of surprises. The paper by Professor Cermak, I think, very much underlines the fact that the nature of our aerodynamic problems is highly conditioned by dynamic influences. This reality must color a lot of our future developments. 
I think the question of the state of the integration of the dynamic aspects into the profession was highlighted by three papers-one by Professor Vickery, one by Dr. Vellozzi and Mr. Cohen, and also the paper by Dr. Chen and Mr. Robertson. I think there wcre several distinct approaches in cvidence here. I might at this point distinguish between what I feel is a code approach, which is, inevitably, a simplified approach-its terms of reference are not altogether the same as the technical aspects of strict engineering design. It has responsibilities to the public, which are embodied in the utility of the code. Five or six years ago, I was asked to contribute a section on dynamic loading which was usable-or hopefully usable - for the Danish Code. This has now been in operation for several years. It was found to bc quite usablc; engineers are finding that it is a manageable concept to deal with and doesn't stretch the ordinary structural engineer's technical background too far. There have been some points which we have to work with further in the Danish Code. One is the question of vortex shedding, and this we're trying to improve on at the moment.

In his paper, Professor Vickery pointed out that the basic concepts of this approach are in reasonable agreement for tall, slender structures with wind tunncl work. The main influence of such a code, which embodies the gust factor, is that, rather than change the level of average loading, if you like, from the total assembly of all structures that we build, the gust factor approach does not move the average value of the wind loading very much. What it does do is discriminate between structures a lot more than present static wind loading codes do. For example, it has tended to relieve the loading significantly on such structures as apartment buildings - which arc very broad and usually relativcly stiff. It has tended to reduce the loading required for such structures from what are our present-day code values. For slender structures, such as smoke stacks, the loadings have increased. And, in consideration of the damage rate, I think this is a consistent development to have happen - as the damage rate certainly would indicate that smoke stacks are quite prone to damage under certain circumstances. So, this has made the question of wind loading a far more dispersed kind of loading than it has hitherto been.

This has not as yet becn adopted very widely by a number of codes. The French codes considcr the Danish approach regarding the gust loading factor, and they did adopt a half-way-house, which was cmbodicd in the Russian Code, but they felt it was too complex. My own feeling is that this is not the case. We in Canada are intending to embody the similar dynamic principle of gust factors.

I think that one of the questions that has to be dealt with very much in the area of structures and codes was referred to, both by Professor Maugh and Mr. Koppes, in their very interesting papers. Professor Maugh referred to construction problems. I think this is a very real question, and it's being neglected in codes.

Another question is the distinction between the skin and the bones. I thought that was a very felicitous phrase. I think it is quite essential that we discriminate between the formulation of the load itsclf, in the first instance, between the skin and the bones. The gust factor approach-along the Danish lines and the new Canadian lines-will be organized around these two aspects. The gust factor will apply, in one sense, to the structure as a whole, and there will be other gust factors entircly for use on the exterior cladding. The reason is, of course, that the size of the structure produces a fairly large averaging effect on the overall loading and it also has resonant amplification. The skin, on the other hand, is exposed to very high local pressures-much higher local pressures-which are not averaged out and the resonant characteristics arc entircly different. The characteristics of the structural components are cntirely different from the characteristics of the structure as a whole.

I think that one other aspect which has bcen referred to this morning-perhaps on the philosophical end, but very important to think about-is the measure of the level of risk. We're using once-in-30year wind loads or once-in-50-year wind loads, and these match up fairly well with what we rcckon to be "working loads" from our experience. The background of this is largely conditioned by history. There was a time when wind loads tended to be organized around the maximum obscrvation on record. Then, people like Mr. Thom came along with statistics, showing that this is not a reasonable basis, bccause records only exist to be broken now and again. It so happened-I think this is a fairly historical evaluation-that the once-in-50-year or the once-in-30-year was an average for the period over which we had records and, thereforc, it was logical to match the wind speeds that were used in design for this kind of return period. But, if you turn to other kinds of loading, such as carthquakc loading-as Professor Clough was referring to this morning-you find that, if you apply the same logic, you end up with a completely ridiculous answer. If people designed for the cxpceted once-in-30-year earthquake load, they 
wouldn't bother about earthquakes at all. What is happening, in a sense, is that we have a margin which is protecting us-which is providing a buffer-between what you might call the most scvere incident that the structural engineer is prepared to entertain. This buffer, or the safety factor, is keeping us out of trouble, because, really, when you talk about a once-in-30-year wind load, you expect it to happenonce in 30 years doesn't mean that you go for 30 years without it. There are reasons to think about this question quite seriously from several points of view. One is that the glass and the structure as a whole do not necessarily deserve to be given the same return period. Some people may think that the loss of glass in a strong wind is a sort of safety valve. There are a lot of philosophical connotations to this problem which I think are worth trying to differentiate between. Another factor here is that for aerodynamic instability where the kind of margin that you have-represented by the once-in-30-year load-is not adequate at all.

Finally, I would like to endorse the remarks that we should have better criteria to deal with in evaluating wind loads. The question of motion has been mentioned several times. These, in many cases, are the most complicated problems and the most difficult problems to overcome. If you can beat these problems adequately, the structure itself will normally have sufficient capacity to deal with strength conditions. There's also the question of fatigue. My impression is that most wind failures, if they do occur, occur through fatigue. And the question of fatigue is always coupled with plastic action because you cannot have plastic action without exposing yourself to fatigue, and to deal with plastic action, purely and simply, without considering how many times the structure is bent back and forth-is to meet only half the problem.

Mr. ThOM:

Dr. Pfrang asked me to say a little about wind damage. I have been making some studies on wird damage, based on data which the Weather Bureau has collected over many ycars-since 1916, as a matter of fact. Several interesting points can be briefly mentioned. Wind damage from large-scale storms, such as hurricanes, extra-tropical cyclones, and also including thunderstorms-which, of course, are small-scale storms, but those in which we can measure the wind speeds, at least--damage from this type of storm runs between five and six hundred million dollars on the average per year. This damage amount fluctuates violently from year to year, and can run up to a billion and a half dollars. The damage from tornados-which are storms in which we cannot measure the wind spceds - runs about one third of the damage from these large-scale storms and thunderstorms. It runs about $\$ 150$ million a ycar, and again, there are large fluctuations from ycar to year.

Of course, much of this damage is to small structures, and literally, the figures aren't too accurate. However, onc can draw some important conclusions from them. At the saine time, we've conducted studies into the mortality-that is, the loss of life-due to these types of storms.

There are some rather interesting facts which I'll be calling attention to in these two studies, and thesc arise from probabilistic considerations. In the case of the large-scalc storm, the damage is constantly increasing from year to year. It is going up exponentially, roughly, as the population increases. This, of course, is due to the fact that the probability of being hit by a storm of this character is proportional to the amount of area that is covered by structures which is proportional to the population. So, this damage has increased from year to ycar and will go on increasing-perhaps with some little reduction, maybe due to design (I don't know what can be done along these lines). Anyway, the fact is that it's increasing, and it must increase based on this increase in probability of structures being struck by such storms.

Now, as far as the loss of life is concerned in these large-scale storms, this has been greatly reduced because of the warning system that's becn put into effect in the last 20 ycars or so. You have observed this yourself; the loss of life in hurricanes, for example, has been reduced to almost no loss of life at all, as compared to 20 or 30 years ago. This, possibly, will increase some along with the probability of being struck by these storms, but, as the warning systems improve-which they, no doubt, will-this will probably keep the loss of life at some reasonable level.

One other thing to consider here, of course, is that the area of the country along the East Coast, where the major part of the population is concentrated, has the largest probability of high winds. So, this contributes a great component to this damage. In the Mid-West, where the population is much more spread-out, the probability of damage is less. On the West Coast, where the population is largc-becoming larger and larger all the time-the probability of high winds is generally very low, so the risk of damage is low.

Now, in the case of tornados, the damage has also increased, because you can't take a house in when there's a warning of a tornado-you can't hide it 
somewhere; it's going to be struck by the tornado if this is the inevitable consequence. The loss of life in tornados also has been greatly reduced by improved warnings. The wind damage, however, continues to rise. It rises in a somewhat different manner, because the high probability of tornados in the United States is in the Mid-West, where the population is, more or less, spread out. But, we can see in the statistics, that, as a result of the migration from farms to cities - which has been large over the past 30 years - the chances of striking urban areas have also increased. Hence, this has been a considerable factor in increasing the damage loss in tornados -that is, the chance of the storm's striking an urban area is much larger than it's striking a single farmhouse. Here again, the situation with respect to the probability is a little different. In the area along the East Coast, where we have the main concentration of population, the probability of tornados is relatively small. On the West Coast, the probability of a tornado is practically zero. So, fortunately, where we have the most sparse distribution of population, we have the high probabilities.

Enough of that ... The striking thing is, I think, that this damage is increasing. And, by the nature of the probabilistic situation, it must constantly increase, except for what can be done in the way of design against this damage.

Now, to actually get to the program: I got the impression from Professor Gill's presentation, that the instrumentation is in pretty good shape. He showed that we have instruments that can be used to measure most of what we anticipate measuring at the present time, and that, if we need to go to higher frequencies, development of such instrumentation could take place-which would take care of the problem. So, I think this is in very good shapethanks, to a large extent, to Professor Gill, himself, who has designed and built many of the instruments that are in use today.

In the second paper by Mr. Singer and Mr. Smith, I thought that a very pessimistic view of things was given. I don't feel that things are quite that bad. It seems apparent, at least, that the wind problem-and the mechanics problem also-divides itself into two parts. One is what might be called a macro-wind--the kind of winds that we now produce for design data. And, superimposed on this is a gust which we determine theoretically and, in some cases, have been able to measure. It's apparent, also, I believe, that the gust is a scale change on the macro-wind, and this is a rather happy set of circumstances, because it makes the statistical theory somewhat simpler. So, if we can determine the gust factors with some degree of accuracy and then superimpose this on a macrowind, I think we would have an estimate which would have a standard error-or relative standard error-that was certainly well within the accuracies of the mechanical design.

I would say another word here-based on some of my own experience. We have used the fastest mile largely because there was record available, and it's the best record we had available-available for a considerable number of years, a requirement for a valid statistical analysis. We might have made a better choice if we had been in a position to start with other instrumentation and we could then have chosen a better measure of the wind. But, one must not forget that this is a climatological problem where, in order to determine probabilities and design values with respect to wind, one must have a fairly long previous record. So, one can't say today, let's change the observation and have a design value tomorrow. If we changed the record, we would have to wait quite a number of years before we could make some reasonable estimate of the probabilities. Now, as to the validity of the present data: we have checked on numerous occasions where there were reports of high winds, in other cases where we were able to add a considerable amount of data to the previous records - and we've found that, in a large percentage of the cases, the records were stable, and that the addition of extra records simply served to enhance the accuracy of the estimates, and did not change the climatological features, namely the statistics of the distribution.

Now, a word about the return period-I don't really like the term "return period;" I like to use "mean recurrence interval" because it explains really what the term means. Mr. Cohen brought up this question this morning, and the question also was raised in Ottawa. No answer has been given to this, and I don't know whether there is an answer to it or not. But, certainly, I think everybody would agree that a 50-year mean recurrence interval is not a measure of the probability as it applies to the structure. As was pointed out by an Austrailian-I think it was Professor Joubert-in Ottawa, that if this were so, there would have been a large number of structures which would have failed over the past hundred years. Well, we haven't experienced this. So, what apparently is happening, and I think this can be demonstrated if one considers a rather simple structure--is that we have enormous factors of safety on the mechanical side. Otherwise, this 50-year design wind just wouldn't be safe. And, we're pretty 
sure that the wind speeds cannot be that far wrong nor can we be that far off on relationship to the static load. Certainly, there are random errors in both, but they couldn't be as far off as this probability must be. So, I think that-as Mr. Cohen said this morningwe should get to a more realistic consideration of the overall probability. As somebody said the other day, we're going to design buildings no matter how much we know, and this is what's gone on in the past. I think a 50-year mean recurrence interval just came to be something that everybody sort of liked. It's been going on for a long time, and if you try to change this in any standard or code, you're going to run into a lot of difficulty. So much for that ...

Mr. Fichtl and Mr. Kaufman gave a very interesting paper which provides a good bit of information on the fine-scale structure of the wind. I think that we need a lot more of this kind of work, particularly at high wind speeds. Most of the spectra that we've had in the past have been determined at low wind speeds by people who are interested in air pollution, and these may not be adequate for the higher speeds. But, I don't think that they're very far off either, because we have other checks with respect to the gust factors, which makes them appear to be rather reasonable.

Finally I would like to comment on wind damage as it relates to the complexity of the structure. I think it is, as Professor Davenport pointed out, if you look at the mechanics of the problem, you'll find that the probability of a concentrated load on the cladding will be much higher than the probability of a load which causes a collapse of the building. The building is made up of a series of components which interact with each other and which produce a convolution of probabilities which makes the chance of failure of the whole structure an extremely small probability. If this weren't so, then we would have much greater collapse damage from wind.

The statistics we get on wind damage are, admittedly, not very good. I don't think anyone can get very good figures on wind damage, but we do the best we can. We've harl a lot of criticism about these figures. Some insurance companies want to do onething with them, and other insurance companies want to do the opposite. For one person, our estimares are too high; for another person the estimates are too low .... What these figures do show is what's inevitable in the probabilistic model-that these are increasing . . . So, I say in both of these studies that the principal use of these estimates is to show the relation from year to year, rather than any absolute value.

From my examination of the data, I would say that a major part of the total consists of a large number of small damages - that is, mainly on the cladding of the buildings. (I'm now talking about thunderstorms, extra-tropical cyclones-the kinds of storms that you would experience, let's say, in Minnesota - excepting tornados.) Now, as far as tornados are concerned, the situation is entirely different. As you know, few structures will withstand a tornado. When a tolnado strikes a building, that's usually the end of it, unless it happens to be a reinforced concrete structure or some similar strong structure; but, even there, there have been cases where a tornado causes a collapse by failure in some particular area of the building. By and large, I would say that, for the large-scale storms, the damage is mainly to the cladding or to relatively small structures.

I remember a Bell Laboratory man saying to me that of 1,500 microwave towers his company had constructed, it had lost three. If you consider the probabilities in this situation, you would expect a lot more failures than that. In other words, those towers are designed to be strong enough to carry the loads. I've wanted to look into those three failures for I have a sneaking suspicion that tornados did the job of destruction. 


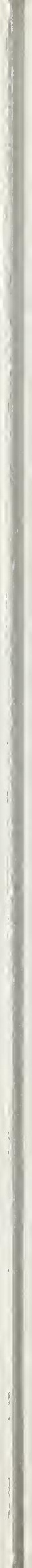




\section{APPENDIX}

Note: The following papers were submitted after the formal program was established. 


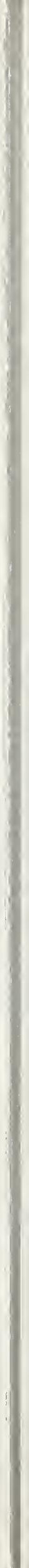




\author{
Gary C. Hart \\ Assistant Professor of Engineering \\ University of California \\ Los Angeles, California 90024
}

\begin{abstract}
A three-dimensional computer oriented stiffness representation of a high-rise building is described. Statistical quantities obtained from aeroelastic models in a boundary layer wind tunnel are combined with the three-dimensional analytical building model to obtain a probabilistic description of the building's response. The response is expressed in terms of the mean and covariance of floor displacements and stresses in the structural members. The procedure is intended to provide a more realistic combination of the aerodynamic and structural behavior of a high-rise building.
\end{abstract}

Key words: Buildings; dynamies; gust loads; matrix analysis; mode shape; probability theory; wind loads.

\section{Introduction}

This paper presents a systematic procedure for combining an aerodynamie high-rise building model with a three-dimensional computer oriented stiffness representation of the building. The building's response is expressed in terms of the mean and covarianee of the floor's displaeements and struetural member's stresses.

A boundary layer wind tunnel is used to model the wind's structure and the topography surrounding the building. Various wind speeds and angles of attaek can be studied. During all sueh studies the aeroelastie model is allowed to translate in two orthogonal direetions and corresponding time histories of response are reeorded. These two orthogonal motion time histories are assumed to be approximations to the aetual building's first two normal coordinate response-time histories. The means, varianees and covariance of these two time histories are ealculated. Then, these statistical quantities are eombined with an advaneed three-dimensional analytical building model to obtain a probabilistic deseription of the building's response. The procedure has assumed that the response of the building's third and higher modes does not signifieantly influence the response and therefore can be negleeted.

The proposed procedure has the following important eharaeteristies: (1) the aerodynamic forees acting on the building are considered in the response, (2) the structural behavior of the building's beams and eolumns ean be modeled using "state of the art" teehniques, and (3) the in-plane rotations of the building's floors are approximately ealeulated by using the building's aetual first two modal vectors.
It is believed that the presented wind design proeedure more realistieally eombines the aerodynamic and structural behavior of a high-rise building. Also, by virtue of the separate modeling of the structural stiffness and the aerodynamie response this procedure is particularly well suited to eontinuously use new future developments in each of these areas.

\section{Dynamical Equations of Motion for the Building}

The first phase of a dynamieal analysis of a high-rise building involves determining the strueture's equations of motion. In sueh a formulation we must first define the building's independent generalized coordinates. Figure 1 shows a simplified building and its assumed generalized coordinates. In general, there will exist three independent rigid body floor displacements per story - two translational and one rotational degree of freedom. For the buildings that are the main coneern of this paper, the translational displaeements of the floors are assumed to be large when compared to the floors' rotational motion. However, this rotational motion can still be expected to significantly affeet the strueture's stresses in the outside frames. Several methods exist for formulating the stiffness matrix of the building $[1,2,3], *$ and it should be emphasized that the aceuraey of the entire wind design proeedure depends on the formulation of the structure's stiffness matrix.

\footnotetext{
* Figures in brackets indicate references at the end of the paper.
} 


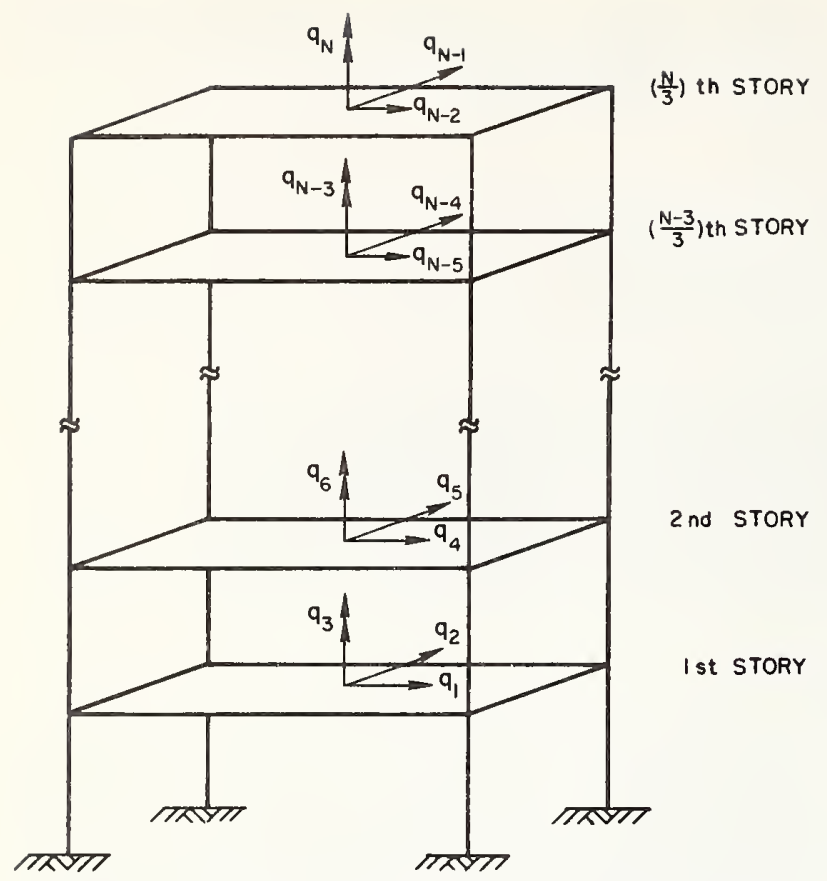

Figure 1. Generalized coordinate (floor) displacements of a building.

If the generalized coordinates of the building are located at the center of mass of each floor, then we can write the building's dynamical equations of motion as

$$
[M]\{\ddot{q}\}+[C]\{\dot{q}\}+[S]\{q\}=\{A\}
$$

Now, if we define the building's normal coordinates as

$$
\{Q\} \equiv[\Phi]^{-1}\{q\}
$$

then the dynamical equation of motion in each normal mode (coordinate) is

$$
\ddot{Q}_{i}+2 \beta_{i} \Omega_{i} \dot{Q}_{i}+\Omega_{i}{ }^{2} Q_{i}=F_{i} ; \quad i=1,2,3, \cdots N
$$

When high-rise buildings are modeled in a wind tunnel, only the first two translational ( $X$ and $Y$ ) modes of the building are modeled. In general such modes will correspond to the first two normal coordinates of the structure. For future discussion it is here established that the $X$ and $Y$ translational modes correspond to the first and second normal modes, respectively. It is also assumed that the building's motion in its other normal modes does not contribute to its response and are assumed to be zero. Therefore, we obtain

$$
\begin{aligned}
\ddot{Q}_{1}+2 \beta_{1} \Omega_{1} \dot{Q}_{1}+\Omega_{1}{ }^{2} Q_{1} & =F_{1} \\
\ddot{Q}_{2}+2 \beta_{2} \Omega_{2} \dot{Q}_{2}+\Omega_{2}{ }^{2} Q_{2} & =F_{2} \\
Q_{i} & =0 ; \quad i=3,4, \cdots N
\end{aligned}
$$

which completely defines the deformed configuration of the building.

Because of this normal coordinate truncation the relation between the generalized and normal coordinate displacements, Eq. (2), simplifies to

$$
\begin{aligned}
\{q\}=\left\{\begin{array}{c}
q_{1} \\
q_{2} \\
\cdot \\
\cdot \\
\cdot \\
q_{N}
\end{array}\right\} & =\left[\begin{array}{cccc}
\Phi_{11} & \Phi_{12} & \cdots & \Phi_{1 N} \\
\Phi_{21} & \Phi_{22} & \cdots & \Phi_{2 N} \\
\cdot & \cdot & & \\
\cdot & \cdot & & \\
\cdot & \cdot & & \\
\Phi_{N 1} & \Phi_{N 2} & \cdots & \Phi_{N N}
\end{array}\right]\left\{\begin{array}{c}
Q_{1} \\
Q_{2} \\
0 \\
\cdot \\
\cdot \\
0
\end{array}\right\} \\
& =\left[\begin{array}{cc}
\Phi_{11} & \Phi_{12} \\
\Phi_{21} & \Phi_{22} \\
\cdot & \cdot \\
\cdot & \cdot \\
\cdot & \cdot \\
\Phi_{N 1} & \Phi_{N 2}
\end{array}\right]\left\{\begin{array}{l}
Q_{1} \\
Q_{2}
\end{array}\right\}=[K]\{\bar{Q}\}
\end{aligned}
$$

Therefore, from Eq. (7) we see that a knowledge of the first two normal mode displacements enables us to completely define all of the building's generalized coordinate displacements. Also, the eigenvector matrix used to relate the normal and generalized coordinate displacements is calculated using the building's stiffness matrix. This matrix is obtained by using the most recent building models and can include such important internal degrees of freedom as column vertical displacements and beam-column joint rotations [2, 4].

Knowledge of the generalized coordinate (floor) displacements enables one to completely define the deformed position of the building. From such a deformed shape the stresses in the building's beams and columns can be expressed in terms of the generalized coordinate (floor) displacements. Let such a relation be written as

$$
\{\sigma\}=[B]\{q\}
$$

The preceding formulation establishes the necessary equations to relate the structure's normal mode displacements to its generalized (floor) displacements and stresses. It is important to emphasize the fact that while the knowledge of the first two normal mode displacements is sufficient information to completely characterize the structure's deformed shape, the error associated with such a shape depends on the validity of the assumption that the contribution of the third and higher normal modes is negligible. This means that the designer- 
analyst must first establish what it is that he wants to caluclate, then calculate the building's eigenvector matrix and decide whether or not the results obtained using only two normal modes are within his desired accuracy criteria.

\section{Wind Tunnel Response of Building}

Present procedures for modeling the wind-cxcited motion of a high-rise building in a wind tunnel attempt to model the building's first two translational mode responses. Figure 2 shows a simplified diagram of the building's wind tunnel model. This model is allowed to move parallel to its two principle axes. This motion is resisted by a physically created spring stiffener and a viscous damper at the base of the model. The dynamical equations of free vibration for the wind tunnel model are

$$
\begin{aligned}
& I_{O X} \ddot{\theta}_{X}+\bar{C}_{X} \dot{\theta}_{X}+\bar{S}_{X} \theta_{X}=0 \\
& I_{O Y} \ddot{\theta}_{Y}+\bar{C}_{Y} \dot{\theta}_{Y}+\bar{S}_{Y} \theta_{Y}=0
\end{aligned}
$$

It should be noted that the wind tunnel model is assumed to be a rigid body supported at its base and the spring stiffness at the base is the only elastic restraint.

Upon studying Figure 1 and Eq. (7), we sce that under certain conditions the generalized coordinates of the wind tunnel model, $\theta_{X}$ and $\theta_{Y}$, are exactly equivalent to the building's first two normal co-

X-DIRECTION MOTION

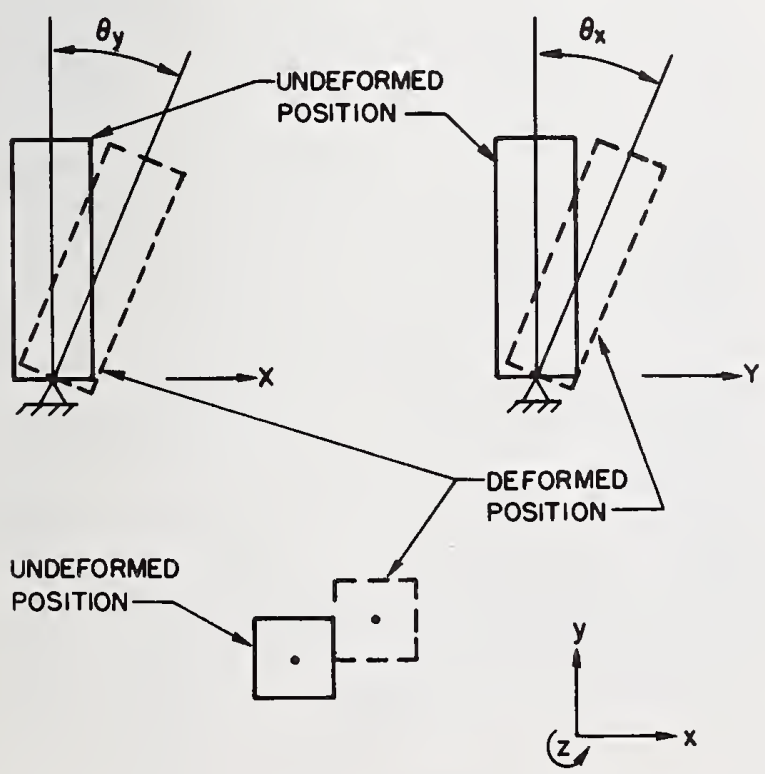

ordinates, $Q_{1}$ and $Q_{2}$. These conditions are: (1) the fundamental mode shapes of the building in its $\mathrm{X}$ and $\mathrm{Y}$ directions are straight lines, and (2) the elements of the first two modal vectors corresponding to the floor rotations are zero. This is shown graphically in Figure 3 for a three-story building. However, in general, these criteria are not exactly satisfied and $\theta_{X}$ and $\theta_{Y}$ only approximate the structure's first two normal modes.

The spring stiffnesses and viscous damping constants of the wind tunnel model are selected such that $\theta_{X}$ and $\theta_{Y}$ approximate $Q_{1}$ and $Q_{2}$, respectively. With such a selection the time histories of building response in the wind tunnel [i.e., $\theta_{X}(t)$ and $\theta_{Y}(t)$ ] are approximations to the actual time histories of first and second mode displacements [i.e., $Q_{1}(t)$ and $\left.Q_{2}(t)\right]$. The accuracy of the approximation can be studied by expanding Eq. (2) for the three-story building in Figure 3,

$$
\begin{array}{rlr}
Q_{1}(t)= & \gamma_{11} q_{1}(t)+\gamma_{12} q_{2}(t)+\gamma_{13} q_{3}(t)+\cdots+\gamma_{19} q_{9}(t) \\
= & \gamma_{11} q_{1}(t)+\gamma_{14} q_{4}(t)+\gamma_{17} q_{7}(t) & \langle X \text {-Direction }\rangle \\
& +\gamma_{12} q_{2}(t)+\gamma_{15} q_{5}(t)+\gamma_{18} q_{8}(t) & \langle Y \text {-Direction }\rangle \\
& +\gamma_{13} q_{3}(t)+\gamma_{16} q_{6}(t)+\gamma_{19} q_{9}(t) & \langle\text { Rotation }\rangle
\end{array}
$$

If the Y-translation displacements and the rotational displacements are zero and if the terms $\gamma_{11}$, $\gamma_{14}$ and $\gamma_{17}$ plot a straight line "mode" shape, then $Q_{1}(t)$ is exactly equivalent to $\theta_{X}(t)$. Otherwise, the displacement measured in the wind tunnel, $\theta(t)$, is an approximate measure of $Q_{1}(t)$. Now we must define what properties of these wind tunnel time histories must be calculated in order that we can accurately describe the behavior of the structure. First we must calculate the mean response of the first and second mode displacements. This is accomplished by using temporal or sample averaging techniques. The mean displacements of the first and second normal coordinates are

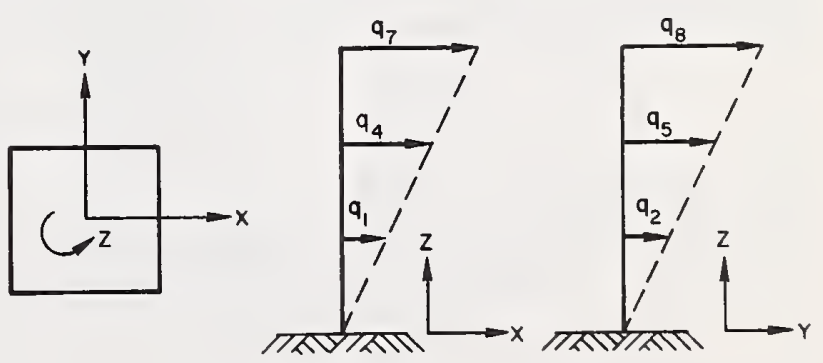

Figure 3. Linear mode shapes of example building.

Figure 2. Wind tunnel representation of building. 


$$
\begin{aligned}
& E\left[Q_{1}(t)\right] \approx E\left[\theta_{X}(t)\right]=\frac{1}{T} \int_{0}^{T} \theta_{X^{-}}(t) d t \\
& E\left[Q_{2}(t)\right] \approx E\left[\theta_{Y}(t)\right]=\frac{1}{T} \int_{0}^{T} \theta_{Y}(t) d t
\end{aligned}
$$

where the length of the time history is denoted by $T$, see Figure 4 .

Second, the variance of the coordinate displacements is of practical engineering importance. This statistical quantity is a measure of the variation about the mean structural response. By taking the square root of the variance, we obtain the standard deviation. A large standard deviation to mean displacement ratio denotes a very oscillatory behavior whereas, a small ratio indicates a nearly static response. To calculate the variance of the first and second normal coordinates, we use the equations

$$
\begin{aligned}
\operatorname{Var}\left(Q_{1}(t)\right) & \approx \operatorname{Var}\left(\theta_{X}(t)\right) \\
& =\frac{1}{\mathrm{~T}} \int_{0}^{T}\left(\theta_{X}(t)-E\left[\theta_{X}(t)\right]\right)^{2} d t
\end{aligned}
$$

$$
\begin{aligned}
\operatorname{Var}\left(Q_{2}(t)\right) & \approx \operatorname{Var}\left(\theta_{Y}(t)\right) \\
& =\frac{1}{T} \int_{0}^{T}\left(\theta_{Y}(t)-E\left[\theta_{Y}(t)\right]\right)^{2} d t
\end{aligned}
$$

Third, the covariance between the first and second normal mode displacements gives an indication of the correlation between the two modal displacements. If the two motions are not very correlated then the covariance term has a small magnitude. The covariance is calculated using the following equation:

$$
\begin{aligned}
\operatorname{Cov}\left(Q_{1}(t), Q_{2}(t)\right) \approx & \operatorname{Cov}\left(\theta_{X}(t), \theta_{Y}(t)\right) \\
= & \frac{1}{T} \int_{0}^{T}\left(\theta_{X}(t)-E\left[\theta_{X}(t)\right]\right) \\
& \cdot\left(\theta_{Y}(t)-E\left[\theta_{Y}(t)\right]\right) d t
\end{aligned}
$$

The variances and covariance can be collected into a $2 \times 2$ matrix called the covariance matrix, it is

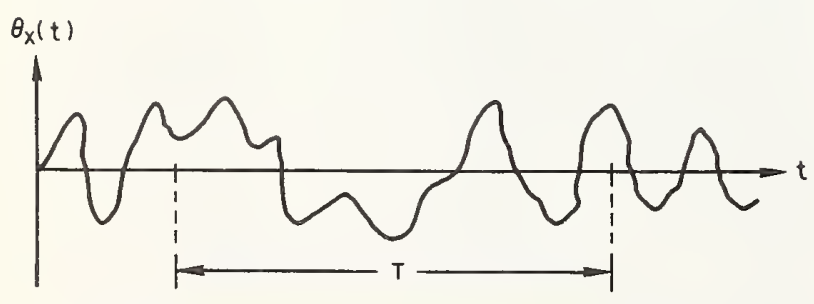

Figure 4. Modal response of wind tunnel model.

$$
\left[V^{\vec{Q}}\right]=\left[\begin{array}{ll}
\operatorname{Var}\left(Q_{1}\right) & \operatorname{Cov}\left(Q_{1}, Q_{2}\right) \\
\operatorname{Cov}\left(Q_{1}, Q_{2}\right) & \operatorname{Var}\left(Q_{2}\right)
\end{array}\right]
$$

The three previously-mentioned statistical quantities enablc us to establish the probability associated with a certain value of building response; (i.e., displacement, shear, or moment). If the building's motion has a Gaussian probability distributed about its mean response, then these statistical quantities completely define the distribution. However, even if the response does not have a Gaussian probability distribution, we can still obtain valuable information about the probability of any response by using Chebyshev's inequality [5].

\section{Probabilistic Response of Analytical Building Model}

In the previous section we calculated the building's mean, variance, and covariance response in its first two normal coordinates. Physically these normal coordinates can be approximated in the wind tunnel; however, from the designer-analyst's viewpoint, they are only a convenient mathematical quantity. The real items of his concern are the shears and moments in the building's beams and columns because it is these generalized forces which determine the building's stress level. Therefore, we must now relate the statistical quantitics we can calculatc to the building's generalized coordinate displacements and forces.

In order to completely define the deformed shape of the building, we must completely define the displacement of all normal coordinates. This is indeed a major task. However, as previously noted, it is sometimes possible to calculate the building's response with a "fair" degree of accuracy by only using two normal modes. In other words, the response of the higher modes is assumed to be zero. It is important to realize that such a two normal mode description of the building's response is only an approximation and may be in error when higher modes significantly contribute to local shears or moments.

Equations (7) and (8) relate the first two normal coordinate displacements to the building's gencralized coordinate (floor rigid body) displaccments and its stresses. Repeating these equations, we write

$$
\{q\}=[K]\{\bar{Q}\} \quad(7, \text { repeated })
$$

and

$$
\{\sigma\}=[B]\{q\}=[B][K]\{\bar{Q}\}=[L]\{\bar{Q}\} \quad(\text { S, repeated })
$$


The statistical propertics of the normal coordinate displaccments calculated as defined in Eqs. (11)(15) can now be used to calculate the corresponding properties of the gencralized coordinate displacements and stresses. The mean values of these quantitics are

$$
E[\{q\}]=[K] E[\{\bar{Q}\}]
$$

and

$$
E[\{\sigma\}]=[L] E[\{\bar{Q}\}]
$$

It can also be shown that the corresponding covariance matrices are

$$
\left[V^{q}\right]=[K]\left[V^{\bar{Q}}\right][K]^{T}
$$

and

$$
\left[V^{\sigma}\right]=[L]\left[V^{\bar{Q}}\right][L]^{T}
$$

The means and covariances calculated as described in (16)-(19) can now be used to completcly define the Gaussian probability distribution or Chebyshev's inequality.

Special attention should be paid to the following items: (1) only two normal modes arc used and, therefore, $\left[V^{\bar{Q}}\right]$ is a $2 \times 2$ matrix; (2) the building matrix is a separate part of the analysis and can be obtained using "statc of the art" techniques; (3) the rotational motion of the floors in the actual building are approximated by using such rotations as they exist in the first two normal modes.

\section{Conclusion}

The procedure outlined in the preceding pages of this paper is intended to help improve the wind design of high-lise buildings whose dynamical bchavior is only slightly asymmetric and whose first two normal mode shapes are nearly linear. By using the building's first two actual modal vectors with the approximate time histories of normal coordinate response we can inprove the accuracy of the computed structural member stresses.

Separatc modcling of the acrodynamic response calculations and the building stiffness characteristies enables us to utilize the continuously improving techniques of cach.

The validity of the procedure for design of highrise buildings which possess the dynamic characteristic assumed herein must be proved by future design and full scalc measurements. Such studies are very strongly recommended.

\section{Nomenclature}

$\{q\}=$ vector of building's generalized corordinate displacements

$[M]=$ diagonal inertia matrix

$[C]=$ damping matrix

$[S]=$ building stiffness matrix

$\{A\}=$ forces corresponding to the gencralized coordinates

$\{Q\}=$ vector of building's normal coordinate displacements

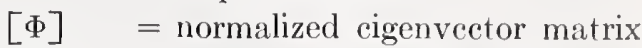

$[\Phi]^{-1}=$ inverse of normalized eigenvector matrix

$\beta_{i} \quad=$ damping in $i^{\text {th }}$ normal mode

$\Omega_{i} \quad=$ natural frequency ( $\left.\mathrm{rad} / \mathrm{sec}\right)$ of $i^{t_{\mathrm{h}}}$ normal mode

$F_{i}=$ force corresponding to the $i^{\text {th }}$ normal coordinate

$N=$ number of generalized displacements for the building, three per story

$\{\sigma\}=$ vector of stresses that exist in the building's beams and columns

$[B]=$ transfer matrix relating generalized displacements and member stresses

$I_{O X}, I_{O Y}=$ mass moment of inertia of the wind tunnel model about an axis passing through the centroid of the base and parallel to the $X$-and $Y$-axis, respectively $\bar{C}_{X}, \bar{C}_{Y}=$ viscous damping of wind tunnel model in the $X$ - and $Y$-direction, respectively

$\bar{S}_{X}, \bar{S}_{Y}=$ rotational spring stiffness at the base of the wind tunnel model in the $X$ - and $Y$ direction, respectively

$[K]^{T}=$ transpose of the matrix $[K]$

\section{References}

[1] Clough, R. W., and I. P. King, "Analysis of ThreeDimensional Building Frames," Publieations, IABSE, Zurieh, Switzerland, Vol. 24, 1964.

[2] Weaver, W. W., and M. F. Nelson, "Three-Dimensional Analysis of Tier Buildings," Jour. of the Struct. Div., Proeeedings of the $A S C E$, Vol. 92, No. ST6, Deeember 1966.

[3] Hart, G. C., "Response of Three-Dimensional Buildings to Multiple Random Wind Forees," Stanford University Teehnical Report No. 92, Dept. of Civil lingineering, Stanford, California, July 1968.

[4] Rubinstein, M. F., and W. C. Hurty, "liffeet of Joint Rotation on Dynamies of Struetures," Jour. of the Engr. Mech. Div., Proeedings of the ASCE, Vol. 87, No. FII6, Deeember 1961.

[5] Parzen, E., Stochastic Processes, Holden-Day, Ine., San Franeiseo, California, 1962. 



\title{
THE ENGINEERING INTERPRETATION OF WEATIIER BUREAU RECORDS FOR WIND LOADING ON S'TRUC'TURES
}

\author{
S. C. Hollister \\ Cornell University \\ Ithaca, New York 14850
}

\begin{abstract}
This paper utilizes the records of the fastest mile as published by the United States Weather Bureau from data obtained at their airport stations, to devclop wind loadings on engincering structures. It analyzes gusts and shows how gust loadings should be dealt with in structural design. Roughness of terrain and extent of cover in rural and urban localities are systemized for enginecring purposes. Occurrence of extreme winds, as studied by the Weather Bureau, is extended to a stage where a practical code may be written. Finally, designing for a given structural life and for a desired level of risk is discussed.
\end{abstract}

Key words: Buildings; climatology; extreme value theory; gust factors; structural engineering; wind loads; wind profile.

\section{The Wind Records}

Wind records in other countries, and older records in this country, simply give the highest velocities observed, without at the same time describing either the terrain or the roughness of cover, or the capability of the instrument; and usually without the duraticn of the reading or the location of the instrument above the ground. Such a record is nearly useless for engineering purposes, since it is not capable of analysis. Responsiveness of the instrument must be known to evaluate accuracy in recording gusts. Time of measurement of gusts is required to deal with gust content. Knowledge of elevation of anemometers above ground is necessary to compute a profile of pressure against a structure. A careful description of the terrain and of the cover along the axis of the principal fetch for a distance of 10 miles is necessary to evaluate the nature of the oncoming windstream, and to compute the relation of velocity and pressure to elevation above ground.

In this country in recent years, records have been made at airports of extreme winds, recorded as the average in miles per hour for the fastest mile of windstream. Such records infer character of terrain and degree of smoothness (although it would be incorrect to assume that the profile of the fetch uniformly justifies use of the seventh-power law). The use of the mean velocity over the fastest mile of wind involves distance and time as well as velocity, and thus gives immediately the opportunity for gust analysis. The Bureau also standardizes the reported observations as at $30 \mathrm{ft}$ above the ground, so that they may be compared statistically with data similarly reported from other places, making possible preparation of probability isotachs and estimates of extreme value forecasts. Thus, such records make possible for the first time the engineering interpretations necessary for designing to resist wind.

This paper deals with the means by which the information needed for rational design may be obtained from the fastest-mile records published by the Weather Bureau.

\section{The Occurrence of Gusts}

A record of wind velocity consists of a more or less rapidly waving line inscribed on a moving tape. Each oscillation is a gust. Means over an interval of one hour do not reveal gusts, but give the velocity of a steady background wind. The ratio of the mean velocity over a short period, as one second, to the hourly mean gives the gust factor $F_{g}$ for that period. Measurements by Giblett, et al. [1]* in 1927-30 at Cardington, England; by Sherlock and Stout [2] in 1933 at Ann Arbor, Mich. ; and by Huss [3] in 1946 at Akron, Ohio, clearly show that the intensity of gusts is greater for shorter than for longer durations; and that for heights to $225 \mathrm{ft}$, at least, the gust factor $F_{g}$ is not a function of height above ground.

Figure 1 shows a plot of the results of Giblett's observations, as further studied by Durst [4], and of Sherlock's study of gusts [5]. Giblett used a Dines anemograph, which according to Scrase [6], loses accuracy at intervals below $10 \mathrm{sec}$. (The 5 -sec. value plotted on Figure 1 shows the tendency to read low on

\footnotetext{
* Figures in brackets indicate literature references at the end of this paper.
} 
in the eeonomieal design of struetures. In the meantime, we must infer that gusts as shown in Figures 1 and 2 have the same probability of oecurrenee.

\section{The Variation of Wind Velocity with Elevation}

It is seen from the foregoing diseussion that an instantaneous profile would have gusts distributed along it and henee would not be a smooth curve. From Figure 1 it is concluded that a mean velocity of 10 min. or more is neeessary to obtain a smooth profile. To sueh a profile an envelope of gusts may be added, thus produeing a synthetic profile of extreme velocities that looks smooth.

Before proeeeding further, it is neeessary to consider the pattern of wind variation well above the ground. Omitting the effect of the roughness of the earth's surfaee on the wind velocity near the ground, the pattern of veloeity oeeurrenees is shown in Figure 3 for the windiest and the ealmest areas of the United States to altitudes to $130,000 \mathrm{ft}$. The lower portions of the eurves are seen to be eoncave downward, from near the ground to about $35,000 \mathrm{ft}$, where strong jet streams are eneountered. These curves are envelopes, and henee are synthetic profiles. It is the form of these eurves that is important here rather than the extreme veloeity, exeept to note that the velocities inerease at an aceelerating rate.

As air moves over a surfaee, it is restrained depending upon the roughness of the surfaee. Turbulenee is eaused not only by the roughness, but by thermal currents rising from the surface; but these eurrents are less signifieant in high winds. Let $Z$ be the distanee from the surface measured normal to it,

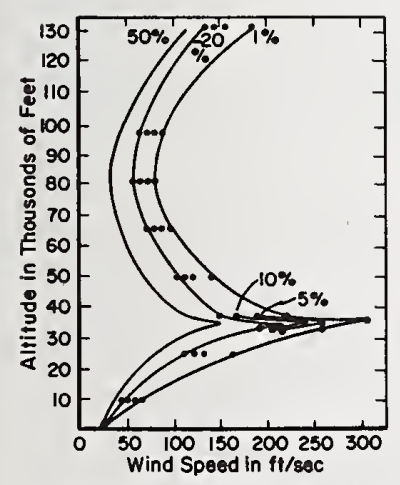

Synthetic Wind Speed Proflloe Exceeded $1 \%$ $5 \%, 10 \%, 20 \%$ ond $50 \%$ of the Winter for the Wind Aroo (Northeoutern Part) of the United Stotes.

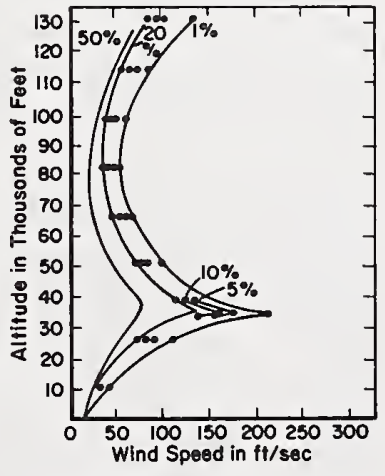

Synthetic wind Speed Protilee Excended $\%$ $5 \%, 10 \%, 20 \%$ and $50 \%$ of the Winter for the Coimet Areo (Northwentern Part) of the Unitud States.
Figure 3. Synthetic probability profiles to $130,000 \mathrm{ft}$ (From USAD handbook ${ }^{9}$. and $v$ the veloeity of the wind at $\%$. If the velocity $v_{A}$ is known at $Z_{A}$, the velocity $v_{B}$ at $Z_{B}$ is

$$
v_{B}=v_{A}\left(Z_{B} / Z_{A}\right)^{1 / n}
$$

where $n$ is a function of the roughness. This is known as Hellman's equation [8]. When the terrain is a plane, either level or very gently inclined, with no trees, shrubs or structures, the experimental evidence gives $n=7$. Large airports at which there is a long unobstrueted fetch over level terrain achieve this condition. (Some airports, with cities upstream, may not achieve this smoothness.) As the roughness inereases, by addition of trees, structures, or irregular terrain, the value of $n$ grows smaller.

As the lower parts of the profiles of Figure 3 meet the ground, they are affeeted by friction so that the profile schematieally has the form shown in Figure 4. At the junetion $T$ of the "free air zone" with the "friction zone," there is a eertain velocity $v_{\iota}$, and a slope $\alpha$ of the tangent to the profile at $T$. Below $T$, the form of the profile is given by Hellman's equation, Eq. (2).

The junetion of the free-air profile at $T$ with the profile of the friction layer imposes two eonditions at

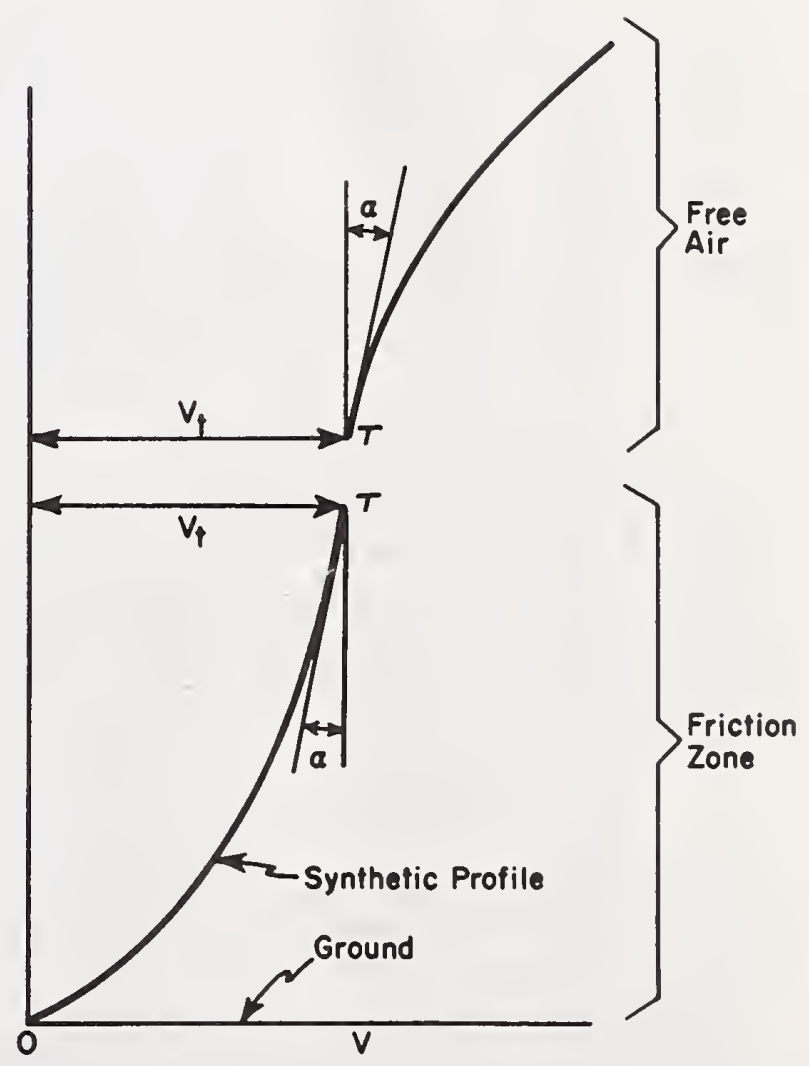

FIgURE 4. Schematic junction of synthetic profile in friction zone with synthetic profile in lower troposphere. 


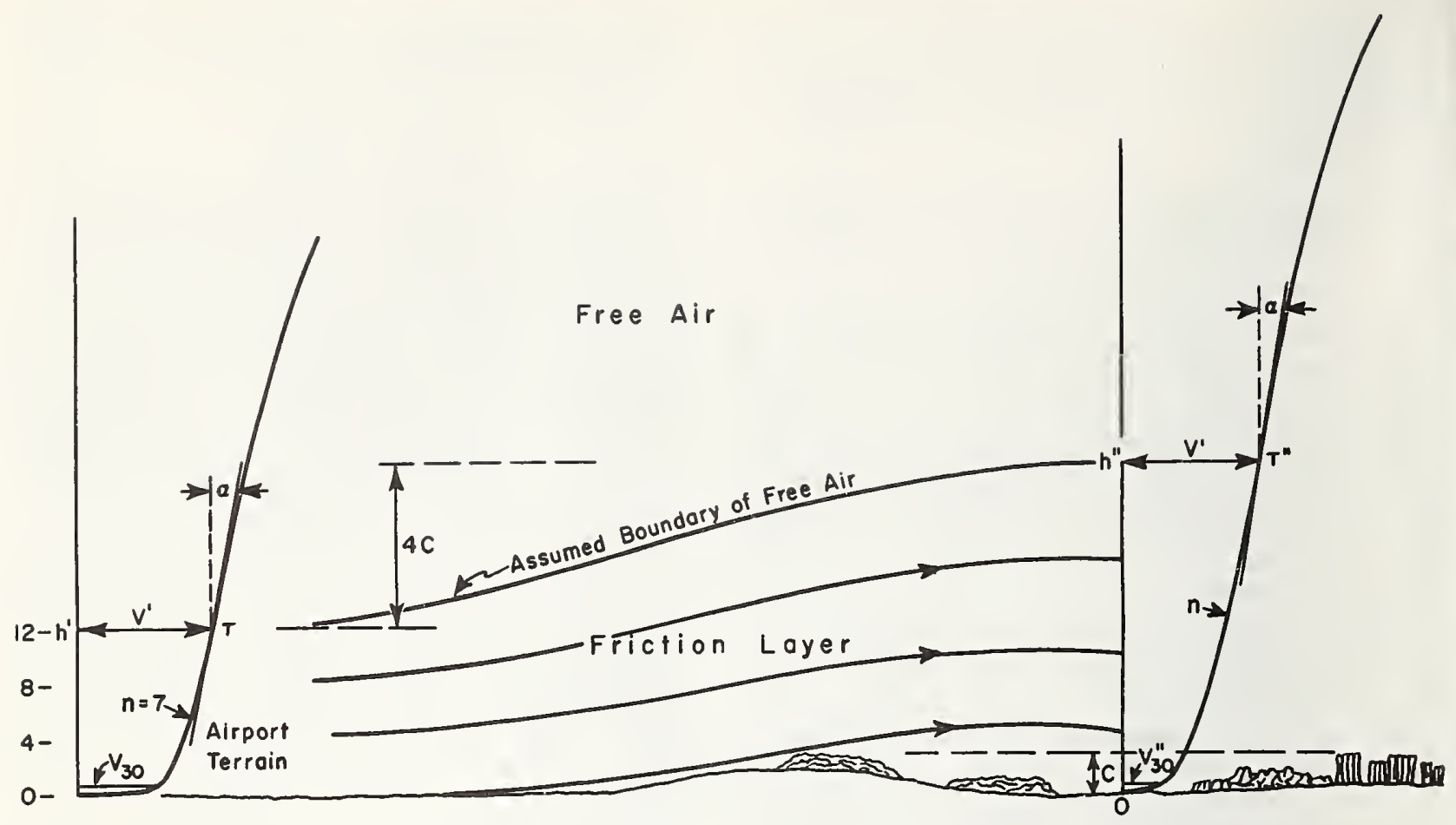

Figure 5. Schematic effect of cover on boundary flow.

$T: v_{t}$ and $\alpha$ are the same at $T$ for both curves. These conditions become important as the wind is followed from the relatively smooth conditions, say, at a large airport, to a rougher terrain and cover, say, over a small town (Fig. 5) [10].

At the airport, shown at the left of Figure 5, we assume $n=7$ in eq. (2), and consider (arbitrarily) that $T$ is at $1200 \mathrm{ft}$. above the ground. The velocity $30 \mathrm{ft}$. above the ground is $v_{30}$, and from this and Eq. (2), $v_{\imath}^{\prime}$ may be found. As the wind moves from left to right, encountering undulating terrain with trees and buildings, turbulence builds up in the friction layer, and at the same time it rises, so that if $C$ is the thickness of the undulations with cover, the boundary of the friction layer at the airport being at a height oh' above the ground, rises to o $h$ " at the small town. A study of data taken over varying roughnesses seems, for engineering purposes, to justify the empirical relation

$$
\overline{o h}^{\prime \prime}=\overline{o h^{\prime}}+4 C
$$

Since $v_{t}^{\prime}$ and $\alpha$ are the same, both at $T$ and $T^{\prime \prime}$, it is possible to compute the profile oT", and thus to determine $n^{\prime \prime}$ and $v_{30}{ }^{\prime \prime}$.

Sherlock [16] and Davenport [10] proposed attaching the friction curve (Fig. 4) to the gradient wind. Sherlock used the Ekman spiral [17] to describe the profile below T. Both Lamb [18] and Taylor [19] had also studied the Ekman problem, which was that of generating vortices in a body of water over the free surface of which wind was blowing. Huss [3] shows that Ekman's, Lamb's and Taylor's work coincided. Sherlock simply inverted the spiral of Ekman.

But the profile $o T$, Figure 4, is not an instantaneous profile. Rather, it must be considered to be an envelope of the gusts that may occur over the same height, and, through the relationship shown in Figures 1 and 2, this envelope is related to a mean hourly curve over this height. The concept here is that the Hellman curve is attached at $T$ with a smooth continuity to an envelope of the gusts occurring in the lower troposphere; and by having at $T$ constant vlues of $v_{t}$ and $\alpha$, for various values of $n$, an interrelated family of curves for the friction layer is derived.

Figure 6 shows the profile with $n=7$ and $v_{30}=100$ mph carried to $1,200 \mathrm{ft}$ above the ground, where $v_{\iota}=169 \mathrm{mph}$. It also shows a family of profiles for various values of $n$ from 7 to 2 , each having the same $v$, and $\alpha$ at $T$ (Fig. 4). The slopes at $T$ were made similar (nearly) by making the chord of the top $100 \mathrm{ft}$ have the same slope, so that $\left(v_{t}-v_{(1-100)}\right)$ was the same for all other curves as it was for $\left(v_{1200}-v_{1100}\right)$ when $n=7$. The value of $n$ was determined from the equation 


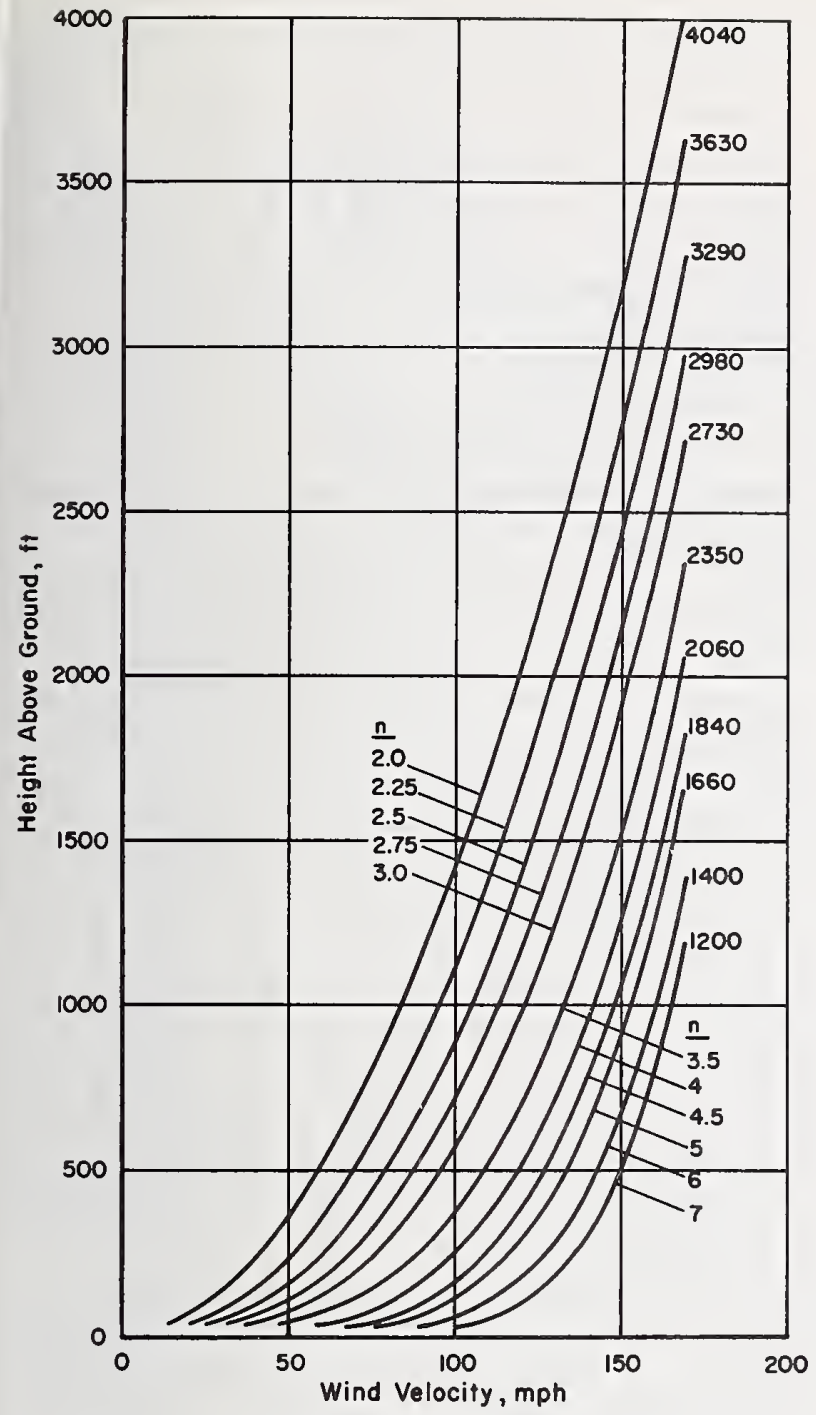

Figure 6. Family of Hellman's eurves based on mean fastest observed mile of $100 \mathrm{mph} 30 \mathrm{ft}$ above ground at airport $(n=7)$.

$\log v_{t}-\log v_{(t-100)}=\frac{1}{n}\left(\log h_{t}-\log h_{(t-100)}\right)$

Figure 6 may be used for locations where $v_{30}$ is other than 100 when $n=7$, by using a simple inultiplier that is easily determined. Figure 7 shows two profiles having the same value of $n$, but with different values of $v_{a}$. From Eq. (2), at any height $h$,

$$
v_{h}=v_{a}\left(\frac{h}{a}\right)^{1 / n} ; \quad v_{h}^{\prime}=v_{a}^{\prime}\left(\frac{h}{a}\right)^{1 / n}
$$

hence,

whence

$$
v_{h}{ }^{\prime} v_{h}=v_{a}^{\prime} / v_{a}
$$




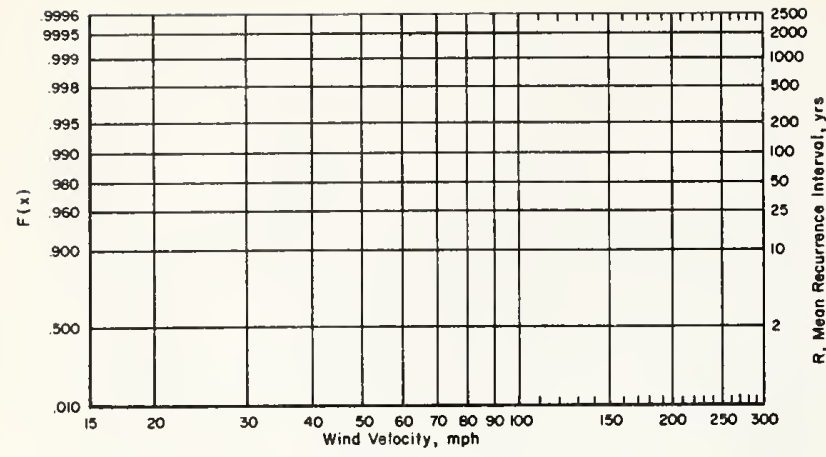

Figure 8. Extreme value probability plotting paper, Fisher-Tippett Type II distribution (after Thom. [11, 12]).

Such a curve (a straight line) would make possible the estimation of the velocity of an extreme wind of any selected mean occurrence interval for the given locality.

The separate annual extreme mile values for a given station may be plotted on the paper of Figure 8 by using the plotting order

$$
p=m /(n+1)
$$

in which $m$ is the order of a given observation, counting from the smallest to the largest observed value (equal readings are arranged serially), $n$ is the total number of observations, and $p$ is the probability $F(x)$.

In his 1954 paper, Thom chose the Type II distribution function

$$
F(x)=e^{-(x / \beta)-\gamma}
$$

to express the probability of a wind speed being less than $x$. The method of maximum likelihood was used to determine the parameters $\beta$ and $\gamma$.

The mean recurrence interval $R$ is given by

$$
R=1 /[1-F(x)]
$$

The probability paper [12] shown in Figure 8 thus shows $F(x)$ on the left margin and $R$ at the right, while the velocity $v$ is shown as the abscissa.

In 1966 Thom [14] published revised isotachs, giving $0.50,0.10,0.04,0.02$ and 0.01 quantities, and the corresponding mean recurrence intervals of 2,10 , 25, 50, and $100 \mathrm{yr}$. for extreme winds in $\mathrm{mph}$ (Figs. 5-13, respectively). The revisions took into account six additional years of records, bringing the average total to 21 and improving the accuracy by about 15 percent.

Based on Thom's method of extreme values and his latest isotachs, the extreme wind probabilities of 83 representative cities have been studied. Figure 14 shows the risk attendant upon the likely occurrence of a wind having a mean recurrence interval once within the expected life of the projected structure. Thus, for an expected 100-yr. life, there is a $10 \%$ risk of one occurrence of a storm of an intensity of a 1,000-yr. mean recurrence interval. Using the paper shown in Figure 8, a straight line plot for a given city, using the isotachs of Thom in Figures 9-13, can be projected to forecast the velocity of a wind having $R=1,000$ yr. By using Figure 2, the hourly mean may be determined, corresponding to the $R=1,000$ wind. Having done this plotting for each of the 83 cities, it was found that they could be grouped into nine classes, as shown in Table I. Table II gives the same information with the cities arranged alphabetically.

The mean hourly velocities for Classes I-IX correspond to fastest mile velocities of $100,105,115$, $125,135,145,155,170$, and $190 \mathrm{mph}$, respectively. These are the 1,000-yr. mean recurrence interval velocities estimated from plotting the isotach values on the paper shown in Figure 8. These classes are also based on mean hourly velocities (see Fig. 2) of 77, 81, $83,95,102,108,115,125$, and $128 \mathrm{mph}$, respectively, likewise at a mean recurrence interval of 1,000 yr. These values are for the airport station at each city at $30 \mathrm{ft}$ above the ground. The effect of terrain and cover within the city itself is not included. Variations in conditions within a given city are likewise matters that must be included in interpreting the basic data given in Tables 1 and 2 .

During the life of a large building, the cover may be materially changed from time to time. As the city gains in the number of tall buildings, the height of cover is increased; and from Figure 6 , it is shown that $n$ is reduced and the average wind velocity acting on a building is likewise reduced. Some cities are undergoing considerable alteration, with the removal of large buildings, altering the exposure and in not a few instances increasing the exposure of a given remaining structure. Thus, in fixing a value for code purposes, it is necessary to provide for such possibility by including the effect of such alteration in city planning.

\section{Determination of Extreme Velocity for Design}

From Figure 1 it is seen that the gust factor $F_{g}$ depends on the duration of the gust. The response of a structure to the gust cannot develop fully unless the gust deviation is long enough to establish the particular regime of the response. The only data available bearing upon the time required to establish 


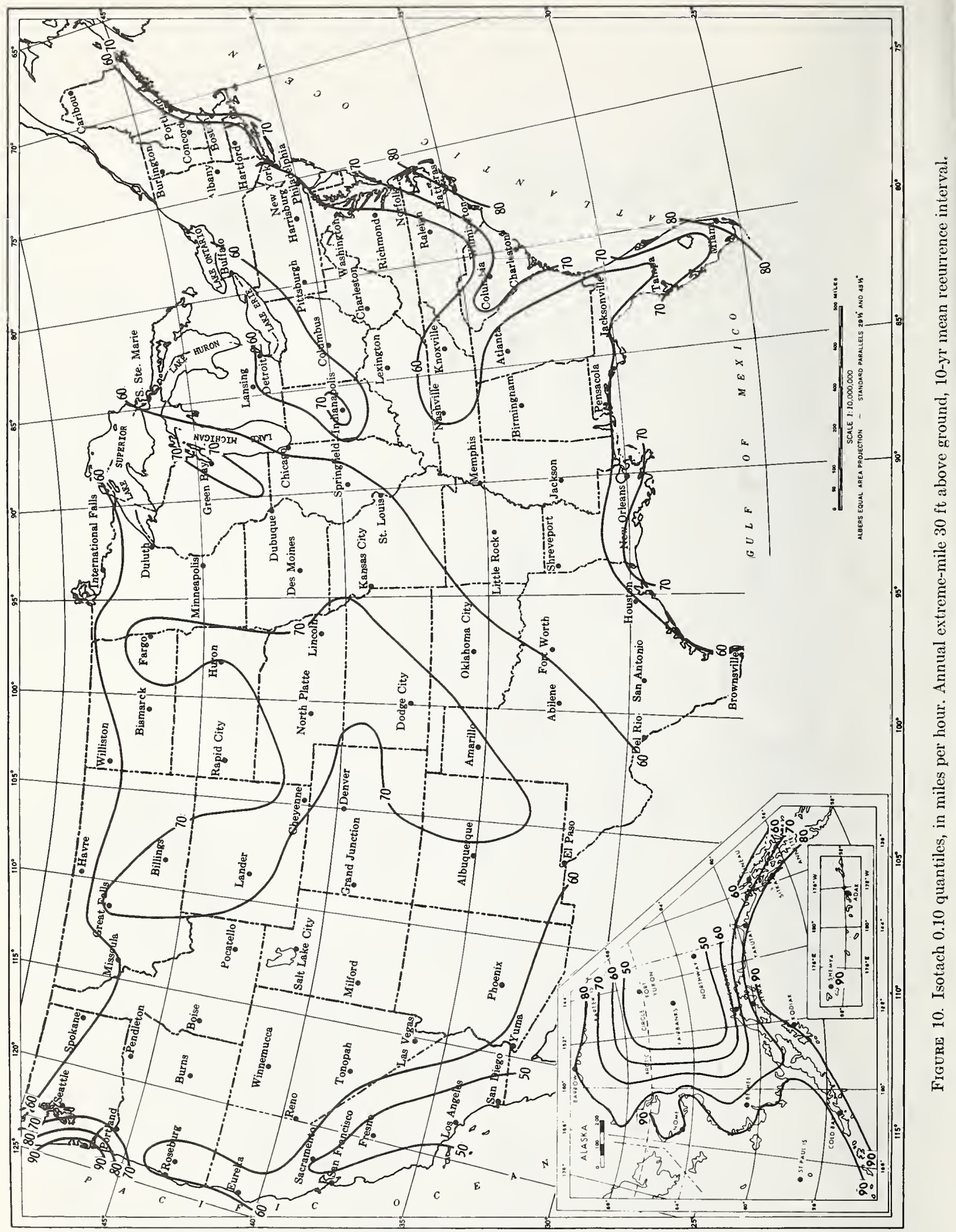




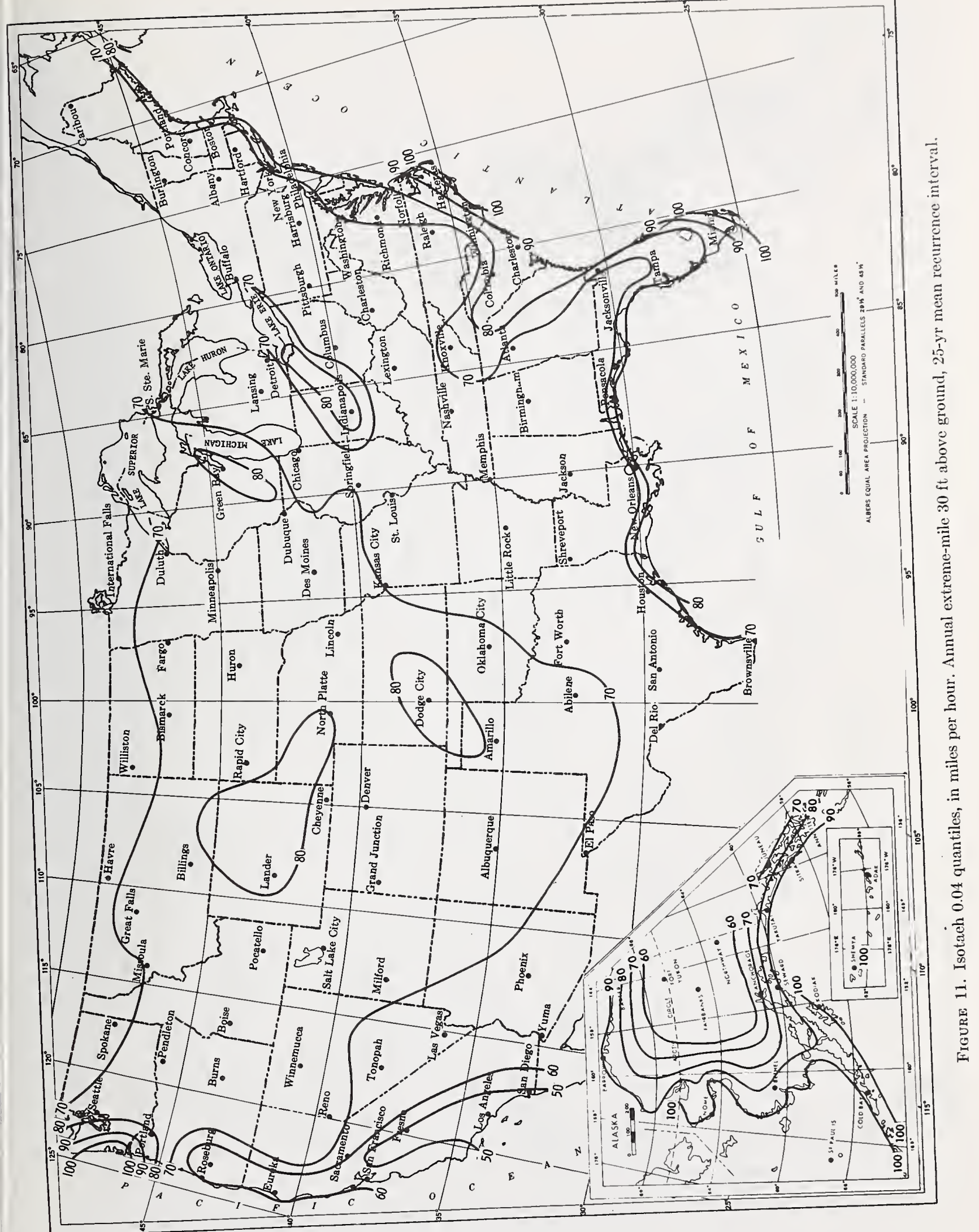




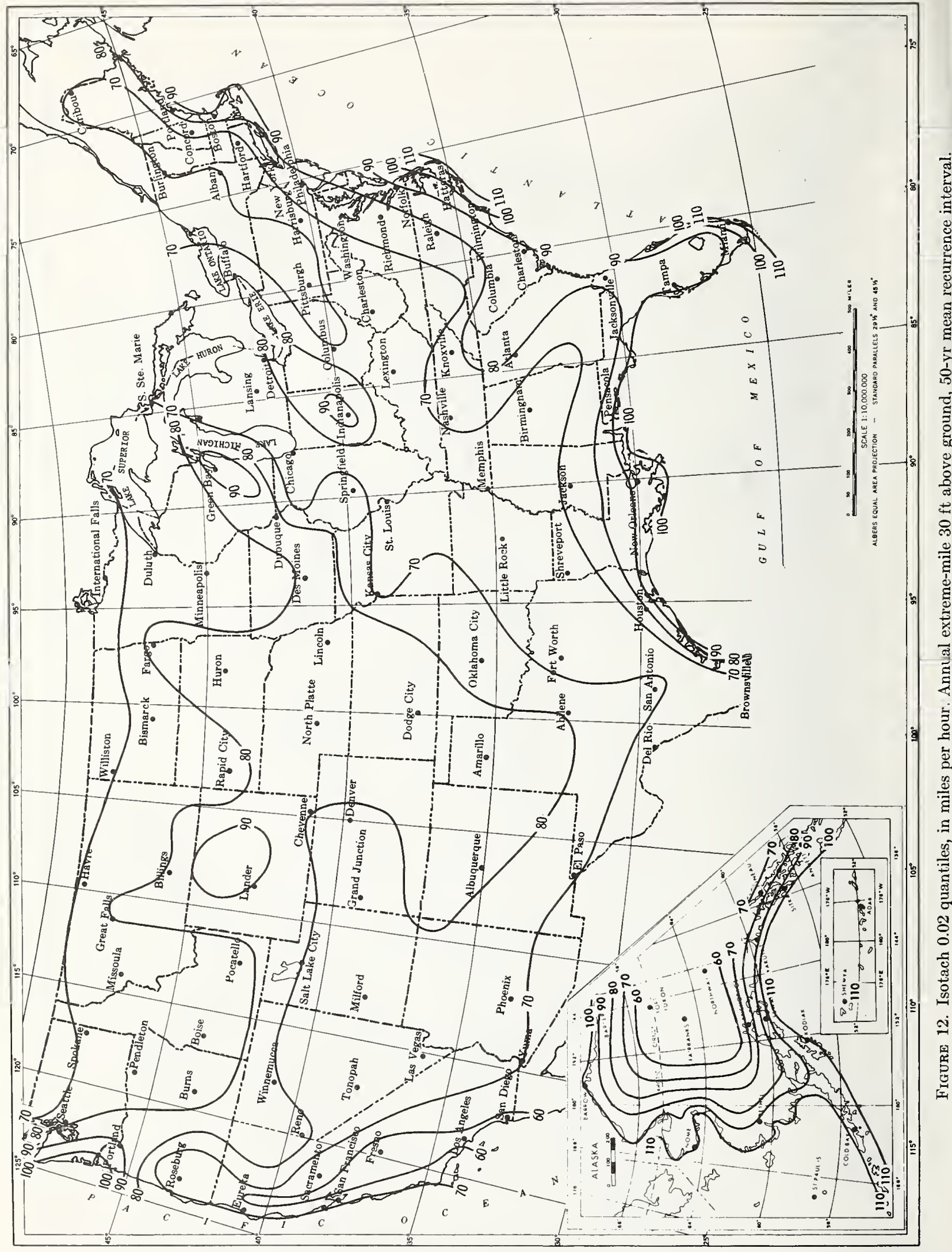




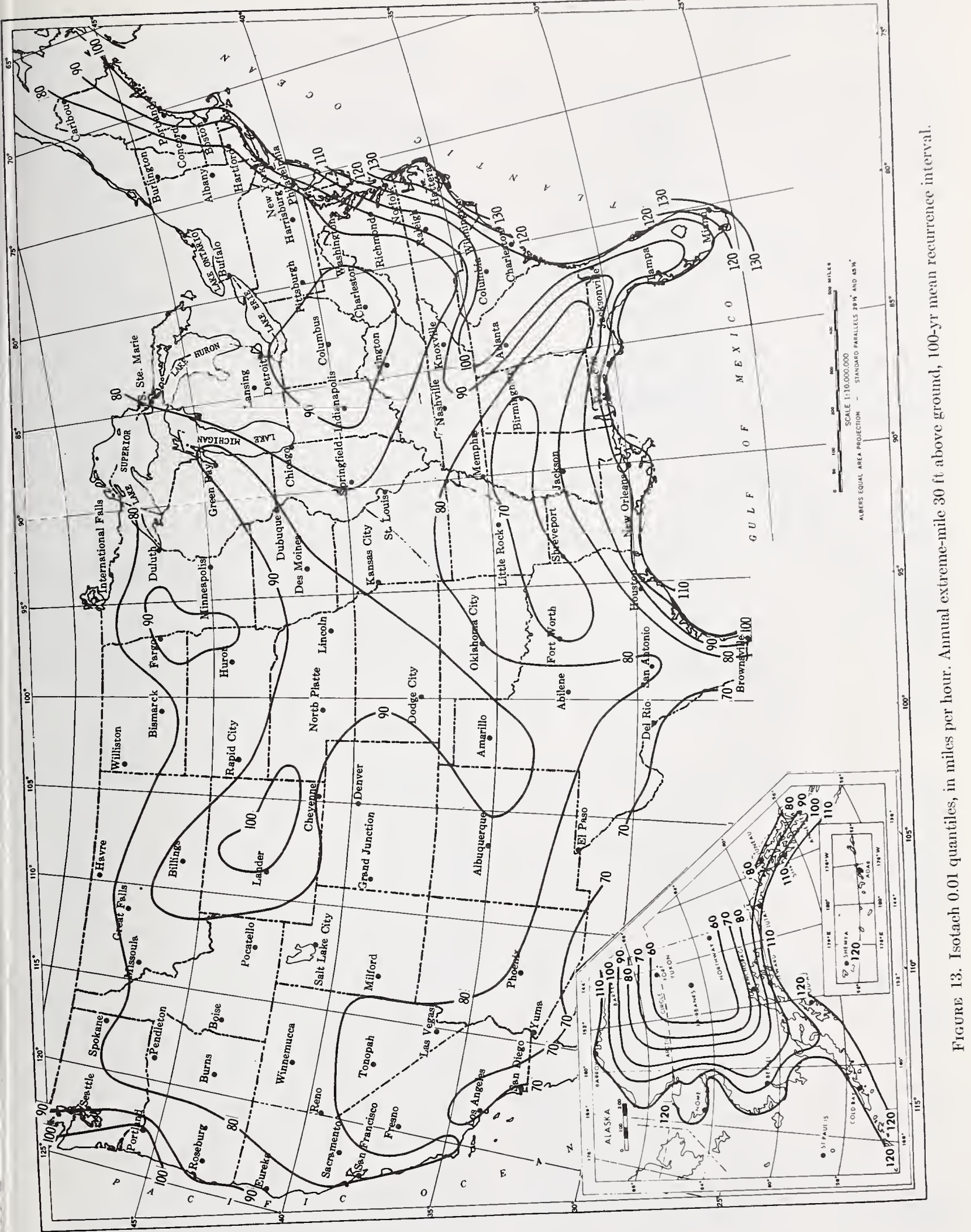


TABLE I. Cities grouped by classes according to extreme fastest mile at 1,000-yr. mean recurrence interval

Class I

(Extreme fastest mile $100 \mathrm{mph}$; fastest hourly mean $77 \mathrm{mph}$ )

Birmingham

Bismarck

Dallas

El Paso

Ft. Worth

Great Falls
Little Rock

Los Angeles

Memphis

Phoenix

San Diego

Shreveport

\section{Class VIII}

(Extreme fastest mile $170 \mathrm{mph}$; fastest hourly mean $125 \mathrm{mph}$ ) Jacksonville

Savannah

\section{Class IX}

(Extreme fastest mile $190 \mathrm{mph}$; fastest hourly mean $128 \mathrm{mph}$ )

Key West

Tampa

Miami

St. Petersburg

Wilmington, N.C.
TabLe II. Cities in alphabetical order with class designation

\author{
Philadclphia \\ St. Louis \\ Spokane \\ Springfield, Ill. \\ Springfield, Mass. \\ Wilmington, Del.
}

Las Vegas

Class III

(Extreme fastest mile $115 \mathrm{mph}$; fastest hourly mean $83 \mathrm{mph}$ )

Amarillo

Buffalo

Cheyenne

Denver

Des Moines

Fresno

Kansas City

Lincoln

Milwaukee

Minneapolis

Nashville

Newark

Class IV

(Extreme fastest milc $125 \mathrm{mph}$; fastest hourly mean $95 \mathrm{mph}$ )

Boston

Charleston, W. Va.

Green Bay

Houston

Knoxville

Class V

(Extreme fastest mile $135 \mathrm{mph}$; fastest hourly mean $102 \mathrm{mph}$ )

Atlanta

Indianapolis

Mobile

\section{Class VI}

(Extreme fastest mile $145 \mathrm{mph}$; fastest hourly mean $108 \mathrm{mph}$ )

Atlantic City

Portland, Maine

\section{Class VII}

(Extreme fastest mile $155 \mathrm{mph}$; fastest hourly mean $115 \mathrm{mph}$ )

Charleston, S.C.

New Orleans
New York City

Omaha

Pendleton

Pocatello

Rapid City

Roanoke

Sacramento

Salt Lake City

San Francisco

Washington, D.C.

Wichita

Louisville

Madison

New Haven

North Platte

Raleigh

Richmond

Seattle

Pittsburgh

Providence

Portland, Oreg.

\section{Norfolk}

Albuquerque

Amarillo

Atlanta

Atlantic City

Baltimore

Birmingham

Bismarck

Boston

Buffalo

Burlington

Charleston, S.C

Charleston, W. Va.

Cheyenne

Chicago

Cincinnati

Cleveland

Concord, N.H

Dallas.

Denver.

Des Moines

Detroit

Duluth

El Paso

Fort Worth

Fresno - -

Great Falls

Green Bay.

Hartford.

Houston

Indianapolis

Jackson, Miss

Jacksonville_

Kansas City.

Key West .

Knoxville

Las Vegas

Lincoln

Little Rock.

Los Angeles

Louisville

Madison

Memphis
VII North Platte

T

\begin{tabular}{l|l|l} 
Class & City & Class \\
\hline
\end{tabular}

II

III

V

Miami

Milwaukee

Minneapolis

VI

II

Mobile.

Nashville

Newark

New Haven

New Orleans

New York City

Norfolk

IV Omaha

III

II

Pendleto

Philadelphia

IV

Phoenix

IV Pittsburgh

II

Pocatello

Portland, Maine

Portland, Oreg

Providence

III

Raleigh

II

Rapid Cit

Richmond

Roanoke

Sacramento

III

I

Saint Louis.

IV

Saint Petersburg-

II Salt Lake City.

IV

V

San Diego

II

San Francisco.

Savannah

VIII

Seattle

Shreveport

IX Spokane

IV

Springfield, Ill

II

Springfield, Mass .

III

Tampa Washington, D.C.

Wichita

IV

Wilmington, Del

IV

Wilmington, N.C.--
IX

III

III

$\mathrm{V}$

III

III

IV

VII

III

VII

IV

III

III

II

I

V

III

VI

VI

V

IV

III

IV

III

III

II

IX

III

I

III

VIII

IV

I

II

II

II

IX

III

III

II

IX 
the regime are due to Farren [15], from research conducted on airplanes. He found that the time required to achieve the regime was that needed by the windstream to pass through a distance equal to eight times the greatest width of the structure.

Suppose a building $500 \mathrm{ft}$ high is to be constructed in Chicago with a life of 100 yrs., in an area where the undulations of the terrain together with the envelope of average building heights is $220 \mathrm{ft}(C=220$ in Eq. (3) and in Fig. 5). Then, from Eq. (3), $o h^{\prime \prime}=2,080$ $\mathrm{ft}$, and we will choose, from Figure $6, n=4$. From Table 2 it is noted that the wind velocity for design is in Class II, with the mean fastest mile of $105 \mathrm{mph}$.

To determine the gust factor, we use Farren's rule of eight times the largest dimension of the building, or $8 \times 500=4,000 \mathrm{ft}$ of windstream, which at $105 \mathrm{mph}$ (154 fps) will pass a point in 26 sec. From Figure 1 we find at a gust duration of $26 \mathrm{sec}$. a gust factor of 1.33 . From Figure 2 we find the mean hourly velocity corresponding to an observed fastest mile of $81 \mathrm{mph}$; and this multiplied by 1.33 gives gust velocity of 108 mph for the fastest $4,000-\mathrm{ft}$ wind within the mean fastest mile of $105 \mathrm{mph}$. This is the velocity at the airport at $30 \mathrm{ft}$ above the ground. At the site we may use the curve for $n=4$ in Figure 6 , by multiplying its abscissas by 1.08 .

The pressure produced by this velocity in standard air, $q$, is (in psf)

$$
q=v^{2} / 391
$$

The velocity at the site is 1.08 times $58 \mathrm{mph}(n=4$, Fig. 6) or $63 \mathrm{mph}$. At $500 \mathrm{ft}$ elevation, it is $69 \times 1.08=$ $75 \mathrm{mph}$. The corresponding pressures are 10 and 14 psf distributed up the building in accordance with the curve $n=4$, Figure 6 .

At leeward corners of buildings negation pressures are experienced equal to the velocity pressure but of opposite sign (suction). Some buildings have lost windows, blown outward. Gusts of short duration can cause loadings of impact suddenness. A window $8 \mathrm{ft}$ high, by Farren's rule, would require $64 \mathrm{ft}$ of wind, which, at $100 \mathrm{mph}$ (or $147 \mathrm{fps}$ ) would pass in $0.44 \mathrm{sec}$. From Figure 1, we find a gust factor of 1.84 . The fastest mile of $100 \mathrm{mph}$ would have a gust factor of 1.30 (Fig. 2); hence the short gust would have a gust factor 1.42 larger. Since the pressure $q$ varies as $v^{2}$ (Eq. (9)), the velocity pressure is doubled.

\section{Selection of Appropriate Gust Factors for Design}

Consider a chimney $800 \mathrm{ft}$ high, with a mean external diameter, $d$, over the top quarter of its height of $25 \mathrm{ft}$; and a mean cover of $200 \mathrm{ft}$. Let the

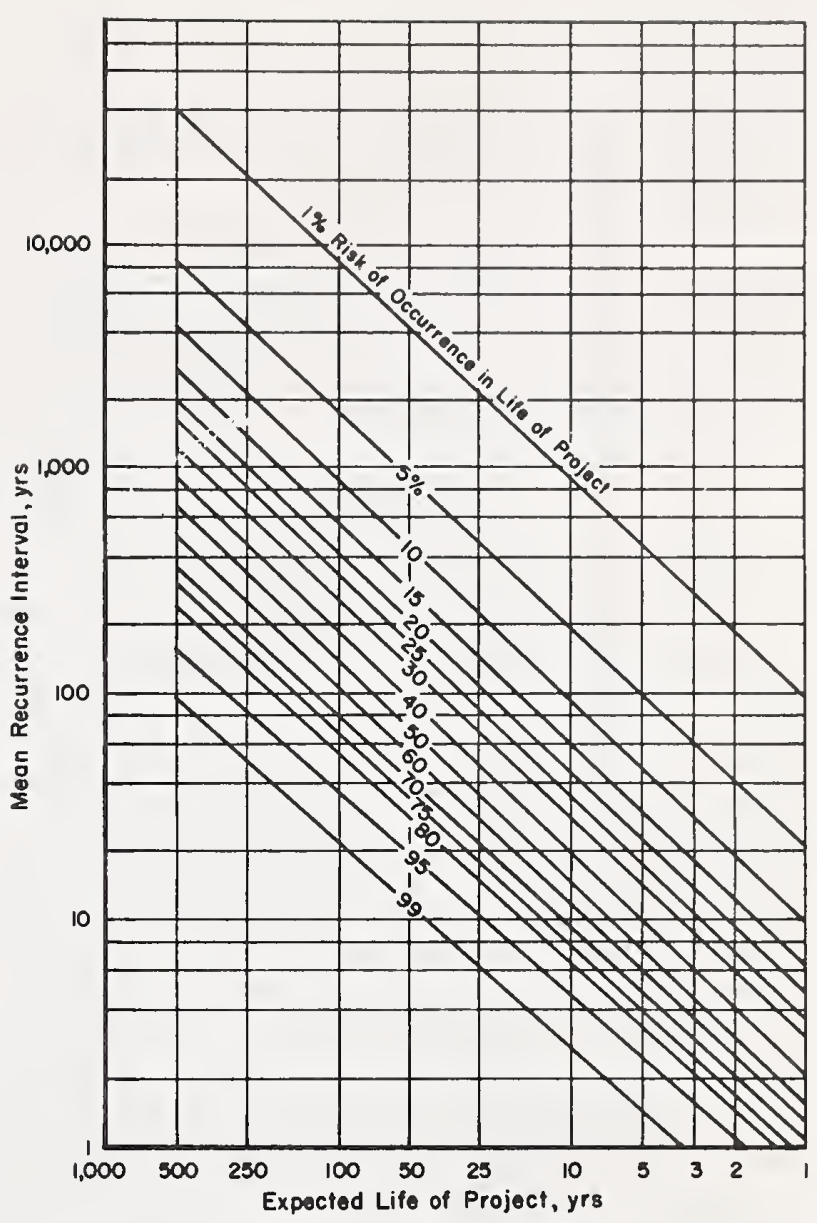

Flgure 14. Risk of occurrence of winds of various mean rccurrence intervals, within the cxpected life of the project.

location be a Class I city (Table 1). For a 100-yr. life, we choose from Figure 14 a value of $R$ of 1,000 yr., for which $v_{30}=100 \mathrm{mph}$. From Figure 6 , we find that with $4 \times 200+1,200=2,000, n=4$, about, when $v_{30}=58$ and $v_{800}=134 \mathrm{mph}$. To estimate the overturning moment, a wind load due to these velocities should be distributed from base to top. The actual velocity pressures, in psf, vary from 9 psf to 45 psf, before taking into account the shape factor.

The collapsing pressure distributed around the ring at, say, the 700 -ft level, is due to a fastest-mile wind of $130 \mathrm{mph}$ (Fig. $6, n=4$ ). If we consider a length of tube of $3 d$, or $75 \mathrm{ft}$, and multiply this by 8 , we have a down-wind travel of $600 \mathrm{ft}$. Since 130 $\mathrm{m} p \mathrm{ph}=176 \mathrm{fps}$, this wind would traverse $600 \mathrm{ft}$ in 3.4 sec. A gust of this duration, according to Figure 1, gives a gust factor $F_{\theta}=1.57$. From Figure 2 , a fastest Inile of $130 \mathrm{mph}$ corresponds to an hourly mean of 98 mph, which, when multiplied by 1.57 , gives a speed of $154 \mathrm{mph}$ for the gust. We must now make a second 
approximation of the gust factor. $154 \mathrm{mph}=226 \mathrm{fps}$, which will traverse $600 \mathrm{ft}$ in $2.67 \mathrm{sec}$. From Figure 1 we correct the gust factor to 1.60 , so that the mean gust velocity will be $1.60 \times 98=157 \mathrm{mph}$. This gives a velocity pressure of

$$
q=v^{2} / 391=157^{2} / 391=63 \mathrm{psf} .
$$

This is the velocity pressure intensity at the stagnation point on the circumference of the cylinder. Having this pressure, the bending moment in a ring $1 \mathrm{ft}$ high may be computed from

$$
M= \pm 0.5 q r^{2}
$$

in which $r$ is the mean radius of the ring.

\section{Setting Stresses for Wind Loads}

Figure 15 shows the plot from the isotachs for the cities Chicago, Jacksonville and Los Angeles. Consider a structure with a life expectancy of 20 yrs. From Figure 14 we see that there is a $10 \%$ risk of a wind having a mean recurrence interval of $200 \mathrm{yrs}$. occurring once in the life of the structure. There is an $18 \%$ risk of a $100-y r$. storm occurring and only a $4 \%$ chance of a $500-y r$. storm occurring. Figure 15 shows that for 100-, 200- and 500-yr. storms, the extreme velocities at $30 \mathrm{ft}$ above the ground at the airport would be 82,90 and $100 \mathrm{mph}$. Suppose the design is based on $82 \mathrm{mph}$ and a $90 \mathrm{mph}$ wind occurs. Then there is an increase of from 100 percent to $110 \%$; and since the pressure increases as the square of the velocity (Eq. (9)), the wind load is increased to $121 \%$. If a 500-yr. storm should occur, the wind load would be increased to $149 \%$. With this information, the design stress for wind can be set to allow a certain encroachment on the factors of safety for the possible rare occurrence of the higher loading.

The flatter curve for Jacksonville produces greater intervals. A building with a projected life of $100 \mathrm{yrs}$. has a $10 \%$ chance of a 1,000-yr. wind and only a $4 \%$ chance of a 2,500-yr. wind occurring in its life. If the

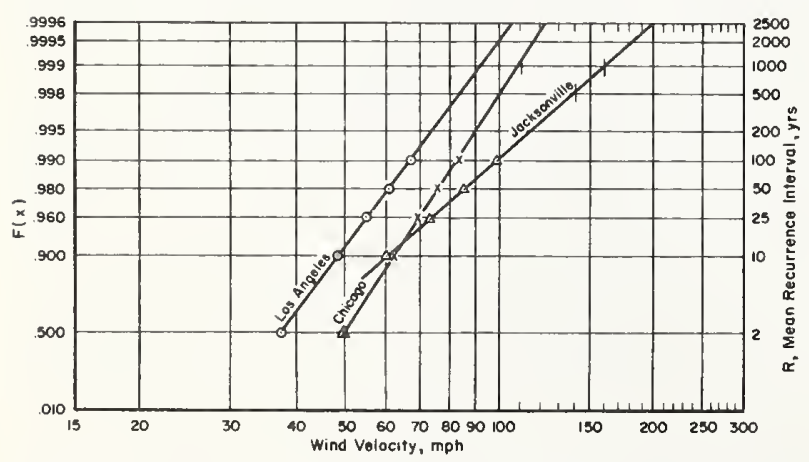

Frgure 15. Plot of probability eurves for threc cities, from isotachs, for extreme velocities at $30 \mathrm{ft}$ above ground at airports. design stress for wind is based on a 1,000-yr. wind, the higher wind would cause an increase in velocity from $100 \%$ (at $161 \mathrm{mph}$ ) to $125 \%$ (at $202 \mathrm{mph}$ ), with a pressure increase of $57 \%$.

\section{References}

[1] Giblett, M. A., et al, The structure of wind over level country. Meteorological Office (Brit.), Geophy. Mcm. No. 54, London, H.M.S.O., 1932, 119 pp., 21 pls.

[2] Sherlock, R. H., and M. B. Stout, Wind structure in winter storms, Jour. Acro. Sci., vol. 5, pp. 53-61, 1937.

[3] Huss, P. O., Relation between gusts and average wind speeds, Danicl Guggenheim Airship Inst., Univ. of Akron, Akron, O., Rept. No. 140, 1946, for U.S. Weather Bureau, Contr. No. Cwb-6103. (Mimeo.)

[4] Durst, C. S., Wind speeds over short period of time, Meteor. Mag., Metcor. Offc. (Brit.), vol. 89, no. 1056, pp. 181-186, July 1960.

[5] Sherlock, R. H., Gust factors for the design of buildings, Int. Assn. Bridge \& Struct. Engrg., vol. 8, pp. 207-230, 1947.

[6] Scrase, F. J., Some charactcristics of eddy motion in the atmosphere, Metcor. Offc. (Brit.), Geophys. Mem. No. 52, London, H.M.S.O., 1930, 16 pp.

[7] Sherlock, R. H. and M. B. Stout, An ancmometer for the study of wind gusts, Engr. Res. Bull. No. 20, Univ. of Mich., Ann Arbor, Mich., May 1931, 36 pp.

[8] Hellman, G., Uber die bewegung der luft in den untersten schichten der atmosphare, Meteorol. Zeit, vol. 34, p. $273,1916$.

[9] Handbook of geophysics for Air Forcc designers. Geophys. Res. Dircetorate, A. F. Camb. Res. Comd., USAF, 1st cd., 1957, pp. 5-17, 5-41.

[10] Davenport, A. G., Wind on structures, Natl. Res. Counc. of Canada, Div. Bldg. Res., Tech. Paper No. 88, Ottawa, Mar. 1960. (No attempt was made to connect the separate curves as has been done in Figs. 4-6.)

[11] Thom, H. C. S., Frcquency of maximum wind spceds, ASCE Proc., vol. 80, 1954, 11 pp.

[12] Thom, H. C. S., Distributions of extreme winds in the Unitcd Statcs, ASCE Trans., vol. 126, 1961, pp. 450-466.

[13] Fisher, R. A., and L. H. C. Tippett, Limiting forms of the frequency distribution of the largest or sinallest member of a sample, Camb. Phil. Soc., Proc. vol. 24, 1928, p. 180.

[11] Thom, H. C. S., New distributions of extreme winds in the United States, Jour. Struct. Div., Proc. ASCE, paper no. 6038, July 1968, pp. 1783-1801.

[15] Farren, W. S., Apparatus for measurement of twodimensional flow at high Reynolds numbers with application to growth of circulation around wing started impulsively from test, Proc., 3rd Int. Cong. Appl. Mech., 1930, p. 329, fig. 8.

[16] Sherlock, R. H., Variation of wind velocity and gusts with height, ASCE Proc., Sep. No. 126, April 1952, pp. 1-26.

[17] Ekman, V. W., On the influenecs of the earth's rotation on occan currents, Arkiv f. mat., astron, ctc., Stockholm, 1905.

[18] Lamb, Horace, Hydrodynamics, 5th ed., Camb. Univ. P'ress, 1924, p. 655.

[19] Taylor, G. I., Eddy motion in the atmosphere, Phil. Trans. Roy. Soc. Lond., Ser. A., Vol. 215, 1915, p. 14. 
Latest developments in the subject area of this publication, as well as in other areas where the National Bureau of Standards is active, are reported in the NBS Technical News Bulletin. See following page. 


\section{HOW TO KEEP ABREAST OF NBS ACTIVITIES}

Your purchase of this publication indicates an interest in the research, development, technology, or service activities of the National Bureau of Standards.

The best source of current awareness in your specific area, as well as in other NBS programs of possible interest, is the TECHNICAL NEWS BULLETIN, a monthly magazine designed for engineers, chemists, physicists, research and product development managers, librarians, and company executives.

If you do not now receive the TECHNICAL NEWS BULLETIN and would like to subscribe, and/or to review some recent issues, please fill out and return the form below.

Mail to: Office of Technical Information and Publications National Bureau of Standards Washington, D. C. 20234

Name

Affiliation

Address

City State $\operatorname{Zip}$

Please send complimentary past issues of the Technical News Bulletin.

Please enter my 1-yr subscription. Enclosed is my check or money order for $\$ 3.00$ (additional $\$ 1.00$ for foreign mailing). check is made payable to: SUPERINTENDENT OF DOCUMENTS. 


\section{Announcement of New Publications in Building Science Series}

Superintendent of Documents, U.S. Government Printing Office, Washington, D.C. 20402

\section{Dear Sir:}

Please add my name to the announcement list of new publications to be issued in the series: National Bureau of Standards Building Science Series.

Name

Company

Address

City State Zip Code

(Notification key $\mathrm{N}-339$ ) 



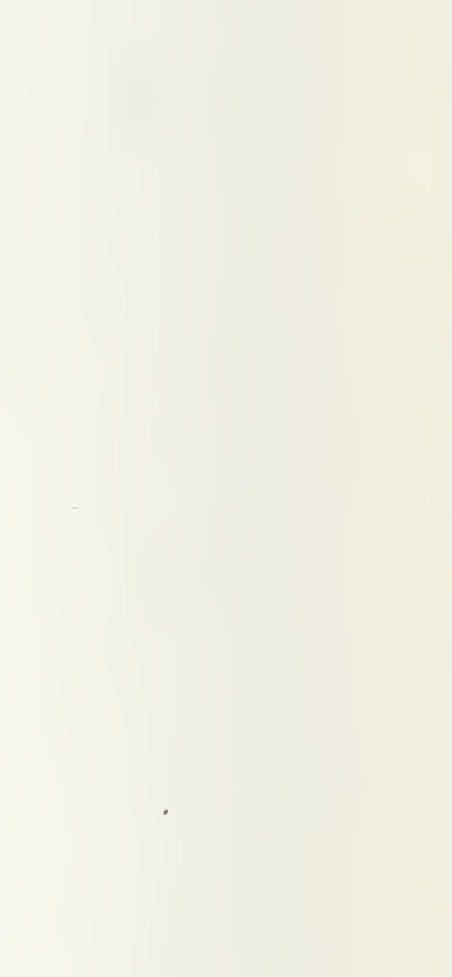


U.S. DEPARTMENT OF COMMERCE

WASHINGTON, D.C. 20230

OFFICIAL BUSINESS

PENALTY FOR PRIVATE USE, $\$ 300$

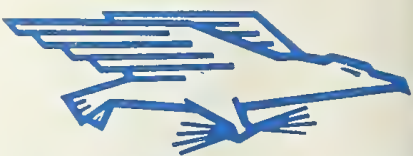

POSTAGE AND FEES PAID U.S. DEPARTMENT OF COMMERCE 